University of Louisville

ThinkIR: The University of Louisville's Institutional Repository

Electronic Theses and Dissertations

$12-2019$

\title{
Laser spectroscopy investigations of jet-cooled metal-containing free radicals.
}

Anam Chandra Paul

University of Louisville

Follow this and additional works at: https://ir.library.louisville.edu/etd

Part of the Physical Chemistry Commons

\section{Recommended Citation}

Paul, Anam Chandra, "Laser spectroscopy investigations of jet-cooled metal-containing free radicals." (2019). Electronic Theses and Dissertations. Paper 3316.

https://doi.org/10.18297/etd/3316

This Doctoral Dissertation is brought to you for free and open access by ThinkIR: The University of Louisville's Institutional Repository. It has been accepted for inclusion in Electronic Theses and Dissertations by an authorized administrator of ThinkIR: The University of Louisville's Institutional Repository. This title appears here courtesy of the author, who has retained all other copyrights. For more information, please contact thinkir@louisville.edu. 


\title{
LASER SPECTROSCOPY INVESTIGATIONS OF JET- COOLED METAL-CONTAINING FREE RADICALS
}

\author{
By \\ Anam Chandra Paul \\ A Dissertation \\ Submitted to the Faculty of the \\ College of Arts and Sciences of the University of Louisville \\ in Partial Fulfillment of the Requirements \\ for the Degree of \\ Doctor of Philosophy in Chemistry \\ Department of Chemistry \\ University of Louisville \\ Louisville, Kentucky
}

December 2019 
Copyright by

Anam Chandra Paul

2019

All rights reserved 



\title{
LASER SPECTROSCOPY INVESTIGATIONS OF JET- COOLED METAL-CONTAINING FREE RADICALS
}

\author{
Anam Chandra Paul \\ A Dissertation Approved on \\ November 6, 2019 \\ by the following Dissertation Committee: \\ Dissertation Director - Dr. Jinjun Liu
}

Dr. Lee Thompson

Dr. Farshid Ramezanipour

Dr. Ming Yu 
This dissertation is dedicated to my parents, parents in law and especially to my lovely wife 


\section{ACKNOWLEDGEMENTS}

At first, I want to acknowledge my advisor, Dr. Jinjun Liu, for all his unparalleled supervision over the last five years. There are so many things I have learned from you, and it is hard to put into words how influential you have been in my transformation from a graduate student into a Ph.D. scientist. Thank you for always reserving me to such a high standard. Sometimes I thought I would only disappoint you because you expected so much, but I was able to reach all the heights you expected with your endless support. Thank you for pushing me to know every number, every equation, and every answer. I am not sure if I always delivered, but just your pushing made me a better scientist. The discussion at group meetings and those "hour" long meetings in your office helped to get attraction in science and motivation to contribute to the scientific community. Thank you very much!

I would like to acknowledge my candidacy exam and dissertation defense committee members, Dr. Lee Thompson, Dr. Farshid Ramezanipour and Dr. Ming Yu. Thanks for being part of the crucial milestones in my graduate school career. The suggestions after my proposal defense helped to find the right way towards my graduation.

I do not even know how to begin to thank the group members in the Liu group. Asmaul Reza, who used to be a senior in my undergrad school back to my 
country, supported me from the first day I arrive in the United States. Initially, gave me a place to live in, cooked all delicious Bengali foods; he introduced me with all the lasers and experimental apparatus in Dr. Liu’s lab. Thank you very much for being so helpful to me “Reza Vai”.

Hamzeh, words cannot express how great of a mentor and colleague you are. I learned a lot from you after you joined "High-Resolution Lab.” You are the best and unique scientist I have ever met. It is rare as a graduate student to have deep knowledge in a combination of three different directions i.e. in organic chemistry, in programming language and in designing experimental apparatus. Your expertise influenced me to learn from very basic. Thanks for helping me in running regular experiment, explaining all the details and finally with the preparation of this dissertation. I am grateful to you my friend!

Abdullah Al-Mamun, a friend of mine for eleven years. We completed our undergrad together from the University of Dhaka, Bangladesh. I was lucky to have you as a mate in my both undergrad and graduate career. You are more than a friend; you are my brother. Thanks for helping me to improve myself as a person by criticizing and pointing out all my flaws. I also would like to acknowledge Arghya, Megan, Aminul, Dhruba and specially Raobo for providing all nessecery software and all of my friends and colleagues in the Chemistry department.

I especially need to thank all the members of my beloved family, for always believing in me. First, to my beautiful wife Rupa Talukder, best find in my life. Thanks for the continuous support of 8 years as girlfriend and 2 years as a wife. Thanks for understanding my late work nights and my stress attacks, for listening to me on a daily 
basis and never complaining. Thanks for the support of the journey of a teenager to a man. To my parents and parents in law, there are not enough words to express the amount of thanks I have for all of you. You are the reason I have succeeded in every walk of my life. Lastly, I want to acknowledge two great people in my life, Nipa Talukder and Jyotirmoy Sarker, for the support and encouragement towards higher education. You guys are awesome!

Finally, I would like to acknowledge to chemistry graduate program assistant Sherry Nalley, Scientific instrument specialist Steven L Reilly and special thanks to Josh Rimmer and UofL machine shop for machining the experimental tool for the research works. 


\begin{abstract}
LASER SPECTROSCOPY INVESTIGATIONS OF JET-COOLED METAL-CONTAINING FREE RADICALS
\end{abstract}

ANAM CHANDRA PAUL NOVEMBER 6, 2019

Metal-containing free radicals are important intermediates in metal-surface reactions and the interactions between metals and organic molecules. Among metalcontaining free radicals monovalent derivatives of alkaline earth metals have been extensively investigated by using spectroscopic techniques in a gas phase ${ }^{1}$, mainly in Broida ovens ${ }^{2}$ or under supersonic-jet-cooled conditions ${ }^{3}$. In the present study, the results of performed spectroscopic investigations of both metal-containing monoalkoxides (CaOR) and metal oxides (MO) radicals have been reported.

Laser-induced fluorescence (LIF) and dispersed fluorescence (DF) spectra of the $\tilde{A}-\tilde{X}$ electronic transition of the calcium CaOR radicals have been obtained under jet-cooled conditions. Complete active space self-consistent field (CASSCF) and coupled-cluster (CCSD) calculations on the free radical were performed to aid the assignment of vibronic transitions observed in the LIF/DF spectra. In addition to dominant spectral features that are well reproduced by vibrational frequencies and 
Franck-Condon (FC) factors calculated $a b$ initio, the pseudo-Jahn-Teller interaction involving the $\tilde{A}$ state induces additional vibronic transitions that are not allowed under the harmonic oscillator approximation. A constant value for the spin-orbit splitting has been observed for all vibrational levels of the $\tilde{A}$ state accessed in the LIF experiment.

Recently, alkaline earth monoalkyl (MR) and monoalkoxide (MOR) free radicals, e.g., $\mathrm{CaCH}_{3}, \mathrm{CaOCH}_{3}$, $\mathrm{SrCH}_{3}$, and $\mathrm{SrOCH}_{3}$, have been proposed as candidates for laser cooling of polyatomic molecules ${ }^{4}$. The implication of the present spectroscopic investigations has been conferred in the context of the proposed scheme of laser-cooling MOR (M=alkaline earth metals) molecules. Accurate determination of Franck-Condon factors (FCFs) is critical to laser cooling. Traditionally, FCFs of MORs are determined in LIF/DF measurements ${ }^{5}$. However, the accuracy of so determined FCFs is limited by the interference of scattering of the excitation laser. In addition, when a pulsed excitation laser is used, the FC-favored transitions may easily be saturated, which leads to under estimated FCFs for these transitions. A direct absorption-based cavity ring-down spectroscopy (CRDS) apparatus is used to find the saturation and corrected measurement of FCFs.

Dark states play a critical role in the laser cooling of atoms and molecules. Population loss due to the relaxation to dark states determines the maximum averaged number of scattering events an atom or molecule can experience. In MOs, the dark electronic state relevant to laser-cooling is the $\widetilde{A}^{\prime 2} \Delta$ state that is in the close proximity to the $\tilde{A}^{2} \Pi$. Since it is “dark”, the $\widetilde{A}^{\prime 2} \Delta$ state doesn't fluoresce significantly and cannot be detected by LIF spectroscopy with a high signal-to-noise ratio (SNR). However, it can be detected using cavity ring-down (CRD) spectroscopy. In an aim to study the dark states, 
$\tilde{A}^{2} \Pi \leftarrow \tilde{X}^{2} \Sigma^{+}$transition of the YO molecule which is one of the only three diatomic molecules that have been laser cooled, was detected using the pulsed-CRD spectroscopy. The sensitivity is comparable to the LIF spectroscopy. A cw-CRD spectroscopy apparatus is under construction, which is expected to increase the SNR by two to three orders of magnitude. 


\section{TABLE OF CONTENTS}

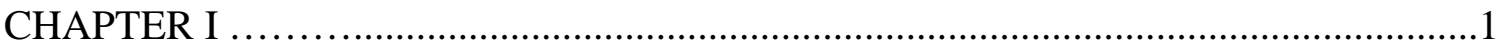

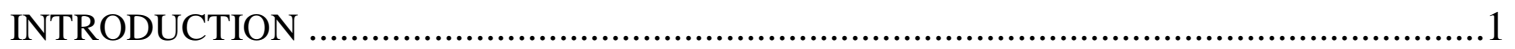

1.1 Metal containing radicals...........................................................................

$1.2 \quad$ Early studies of metal-containing free radicals........................................................

1.3 Molecular beam and supersonic jet expansions..............................10

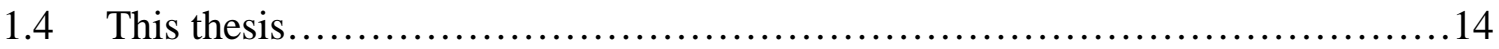

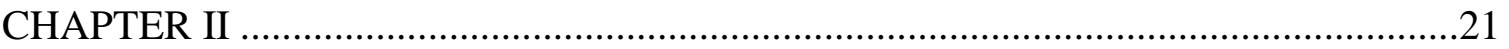

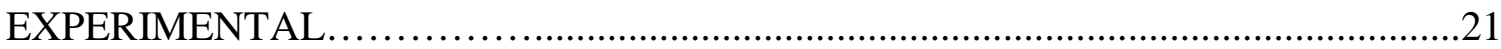

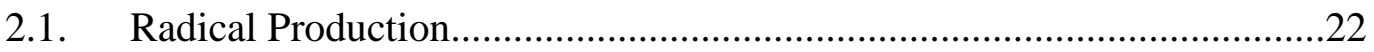

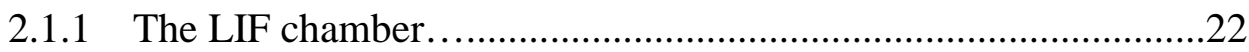

2.1.2 Laser ablation and supersonic nozzle..........................................24

2.1.3 Supersonic jets and molecular beams..............................................31 
2.2 Electronic spectroscopy

2.2.1 Laser-induced fluorescence (LIF) spectroscopy. .33

2.2.2 Dispersed-fluorescence (DF) spectroscopy. .36

2.2.3 Experimental synchronization. .37

2.3 Cavity ring-down (CRDS) spectroscopy. 38

2.3.1 Principle of cavity ring-down spectroscopy .38

2.3.2 Experimental setup. 42

CHAPTER III 59

\section{LASER-INDUCED FLUORESCENCE AND DISPERSED-FLUORESCENCE SPECTROSCOPY OF JET-COOLED CALCIUM METHOXIDE} .59

3.1.1 Experimental (LIF and DF) ...........................................................65

3.1.2 Experimental (Cavity ring-down spectroscopy) ........................70

3.2 Quantum chemical calculation........................................71

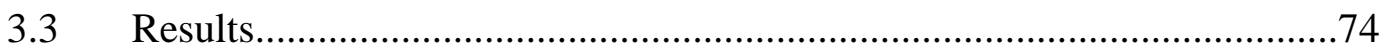

3.3.1 LIF spectrum..........................................................................

3.3.2 DF spectra obtained by pumping origin transitions................76

3.3.3 DF spectra obtained by pumping other vibronic bands...........78

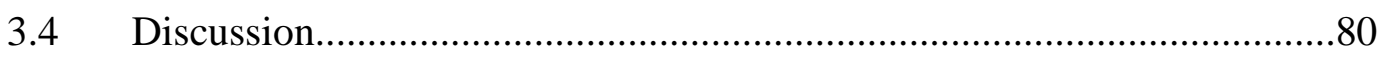


3.4.1 Comparison of CASSCF and CCSD calculations for the $\tilde{A}$ and

$\tilde{X}$ states with experiment...................................... 80

3.4.2 Corrections to the quantum chemical results....................81

3.4.2.1 Vibronic coupling within and between the $\tilde{A}$ and $\tilde{B}$ states.........82

3.4.2.2 Spin-orbit coupling......................................... 86

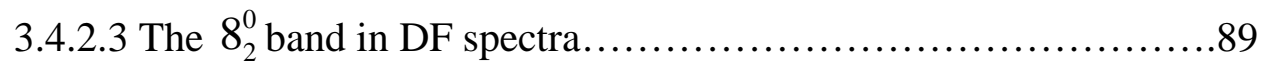

3.5 Comparison with previous experimental values of VBRs.................92

3.6 Implications to direct laser cooling of $\mathrm{CaOCH}_{3}$ and TRSV ...............94

3.7 Conclusions..........................................................................................95

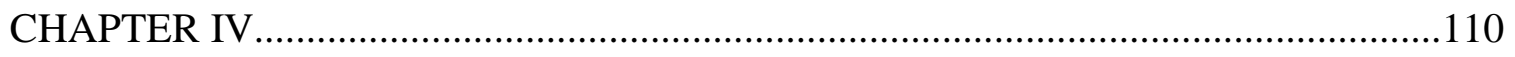

THEORY OF PSUDO-JAHN-TELLER MOLECULES ...........................................110

4.1 Estimated relative errors of experimentally determined FC- factors.....110

$4.2 \quad$ Vibronic Hamilton ............................................................................... 113

Intensity calculation............................................... 118

4.4 Vibronic and SO interaction between the $\tilde{B}^{2} A_{1}$ and $\tilde{A}^{2} E$ states...........120 
CHAPTER V

\section{LASER-INDUCED FLUORESCENCE AND DISPERSED FLUORESCENCE} SPECTROSCOPY OF JET-COOLED CALCIUM ETHOXIDE..................................138

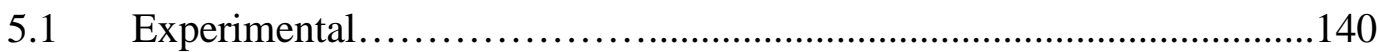

5.2 Quantum chemical calculations.....................................142

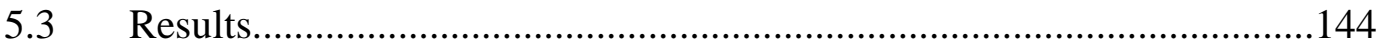

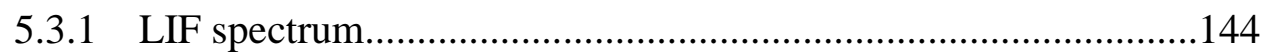

5.3.2 DF spectra obtained by pumping the origin transitions................146

5.3.3 DF spectra obtained by pumping other vibronic bands ..........148

5.4 Implications to direct laser cooling of $\mathrm{CaOC}_{2} \mathrm{H}_{5}$ and TRSV .................150

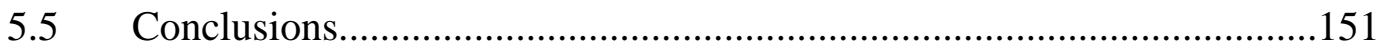

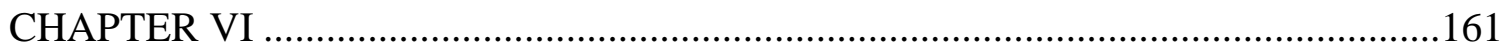

LASER-INDUCED FLUORESCENCE AND DISPERSED FLUORESCENCE SPECTROSCOPY OF JET-COOLED CALCIUM ISOPROPOXIDE.............................161

6.1 Quantum chemical calculations.............................................................164

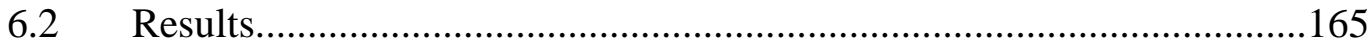

6.2.1 LIF spectrum...................................................................165

6.2.2 DF spectra obtained by pumping the origin transitions................167 
6.3 Conclusions. .169

CHAPTER VII 176

\section{CAVITY RING-DOWN SPECTROSCOPY OF JET-COOLED YITTRIUM OXIDE}

(YO). 176

7.1 Experimental. 178

7.2 Results and Discussion 181

7.2.1 Electronic structure of YO. 181

7.2.2 Rotational assignment of the bands.... .182

7.3 Effective Hamiltonian.................................................................184

7.4 High-temperature spectra recorded with slit-jet expansion..............187

7.5 Improvement of the resolution and sensitivity......................188

7.6 Conclusion..................................................... 190

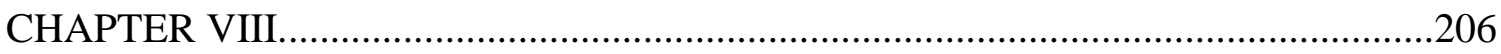

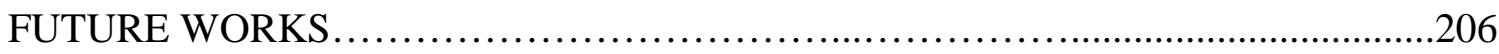

8.1 Measurement of the spin-ro-vibronic structure of the ground and the firstexcited electronic states of MOHs. .209

8.2 Detection of the dark states of MOHs .211 
8.3 Measurement of FCFs.............................................212

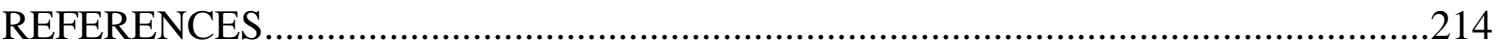

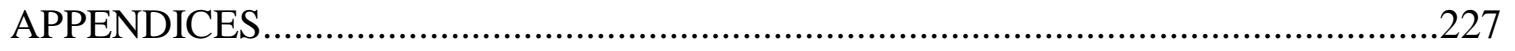

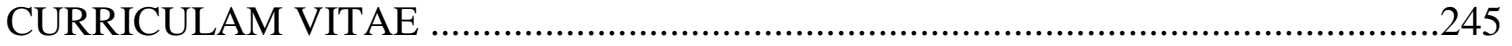




\section{LIST OF TABLES}

Table 2.1. Truth table of JK flipflop.

Table 2.2. Motor driver module motor driving modes.

Table 2.3. Delay timing used in LIF/DF experiment....................................................48

Table 2.4. Delay timing used in CRDS experiment

Table 3.1. Geometric parameters, rotational constants and $\tilde{A}-\tilde{X}$ excitation energy of the $\mathrm{CaOCH}_{3}$ radicals. 97

Table 3.2. Harmonic vibrational frequencies of $\mathrm{CaOCH}_{3}$ in $\tilde{X}^{2} A_{1}$ and $\tilde{A}^{2} E$ states (in $\mathrm{cm}^{-}$

${ }^{1}$ ). Vibrational modes are numbered following the Herzberg convention.................98

Table 3.3. Relative intensities (proportional to the FC factors assuming BOA) $\tilde{A}^{2} E \leftarrow$ $\tilde{X}^{2} A_{1}\left(v^{\prime \prime}=0\right)$ vibronic excitation transitions. The total intensity is normalized to unity

Table 3.4. Vibrational branching ratios (VBRs) for $\tilde{A}^{2} E\left(v^{\prime \prime}=0\right) \rightarrow \tilde{X}^{2} A_{1}$ vibronic transitions. The total intensity is normalized to unity. .100

Table 3.5. Comparison of VBRs for the $\tilde{A}^{2} E \rightarrow \tilde{X}^{2} A_{1}$ emission transitions determined in the present work reported in Ref. 13 101 
Table 3.6. Calculated relative intensities for the $\tilde{A}^{2} E \leftarrow \tilde{X}^{2} A_{1} 0_{0}^{0}$ and $8_{0}^{1}$ excitations transitions. 102

Table 4.1. Harmonic frequency and Jahn teller coupling parameters in cylindrical coordinates. He is the electronic potential at the symmetric configuration, which includes Coulomb interaction, exchange interaction etc. .121

Table 4.2. Harmonic frequencies, linear and quadratic Jahn-Teller coupling constants and pseudo-Jahn-teller coupling constants calculated at EOMIP-CCSD level using a cc-PVTZ basis. All values are in the unit of $\mathrm{cm}^{-1}$.

Table 4.3. Cross-quadratic coupling constants calculated at the EOMIP-CCSD level using cc-PVTZ basis. All values are in units of $\mathrm{cm}^{-1}$.... 124

Table 4.4. Bilinear coupling constant calculated at EOMIP-CCSD level using a cc-PVTZ basis. All values are in units of $\mathrm{cm}^{-1}$ 124

Table 4.5. Eigenvalues and coefficients of basis functions contributing to eigenvectors $\left|\widetilde{\Psi}_{\tilde{A}}^{n}\right\rangle$ for the parameter set in table 4.2 excluding the spin-orbit coupling between $\tilde{A}$

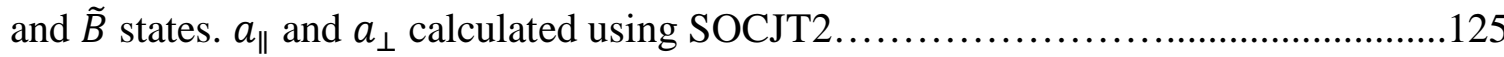

Table 4.6. Eigenvalues and coefficients of basis functions contributing to eigenvectors $\left|\widetilde{\Psi}_{\tilde{A}}^{n}\right\rangle$ for the parameter set in table 4.2 excluding the spin-orbit coupling between $\tilde{A}$

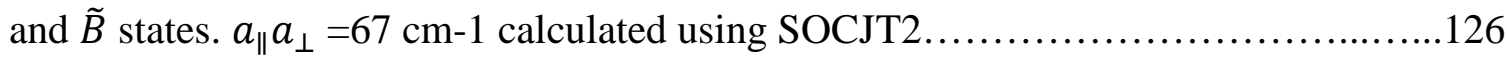

Table 4.7. Electronic transition dipole moment from $\Lambda_{x}$ to $\Lambda_{A}$ and $\Lambda_{B}$ states. .127 
Table 4.8. Intensity calculations for the excitations spectra using $\tilde{X}$ state normal coordinates for the parameters set in table 4.2 excluding the spin-orbit coupling between

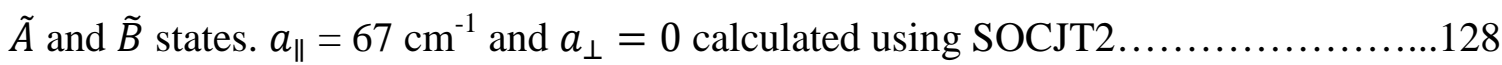

Table 4.9. Intensity calculations for $\tilde{X} \leftarrow \tilde{A} 0_{0} E_{1 / 2}$ emission spectra using $\tilde{X}$ state normal coordinates for the parameter set 4.2 excluding the spin-orbit coupling between $\tilde{A}$ and $\tilde{B}$ states. $a_{\|}=67 \mathrm{~cm}^{-1}$ and $a_{\perp}=0$ calculated using SOCJT2......................129

Table 4.10. Intensity calculations for $\tilde{X} \leftarrow \tilde{A} 0_{0} E_{1 / 3}$ emission spectra using $\tilde{X}$ state normal coordinates for the parameter set 4.2 excluding the spin-orbit coupling between $\tilde{A}$ and $\tilde{B}$ states. $a_{\|}=67 \mathrm{~cm}^{-1}$ and $a_{\perp}=0$ calculated using SOCJT2 $2 \ldots \ldots \ldots \ldots \ldots \ldots \ldots \ldots$

Table 4.11. Intensity calculations for the excitations spectra using $\tilde{X}$ state normal coordinates for the parameters set in table 4.2 excluding the spin-orbit coupling between

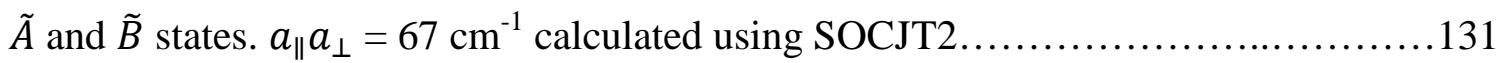

Table 4.12. Intensity calculations for $\tilde{X} \leftarrow \tilde{A} 0_{0} E_{1 / 2}$ emission spectra using $\tilde{X}$ state normal coordinates for the parameter set 4.2 excluding the spin-orbit coupling between $\tilde{A}$

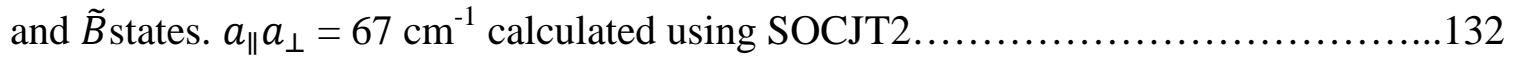

Table 4.13. Intensity calculations for $\tilde{X} \leftarrow \tilde{A} 0_{0} E_{3 / 2}$ emission spectra using $\tilde{X}$ state normal coordinates for the parameter set 4.2 excluding the spin-orbit coupling between $\tilde{A}$

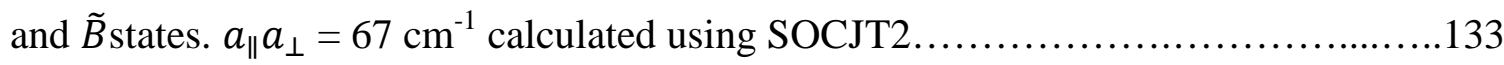


Table 4.14. Cubic coupling constant $\left(f_{i j k}\right)$ in Cartesian representation for the $\tilde{X}$ state of $\mathrm{CaOCH}_{3}$. The values are calculated using VPT2 at the EOMEA-CCSD/cc-PVTZ level of theory. All values are in $\mathrm{cm}^{-1}$ 134

Table 4.15a. Quadratic coupling constant $\left(f_{i j k l}\right)$ in Cartesian representation for the $\tilde{X}$ state of $\mathrm{CaOCH}_{3}$. The values are calculated using VPT2 at the EOMEA-CCSD/cc-PVTZ level of theory. All values are in $\mathrm{cm}^{-1}$. 135

Table 4.15b. Quadratic coupling constant $\left(f_{i j k l}\right)$ in Cartesian representation for the $\tilde{X}$ state of $\mathrm{CaOCH}_{3}$. The values are calculated using VPT2 at the EOMEA-CCSD/cc-PVTZ level of theory. All values are in $\mathrm{cm}^{-1}$. 136

Table 4.16. Frank Condon factors $f v^{\prime} v^{\prime \prime}=\left|\left\langle v^{\prime} \mid v^{\prime \prime}\right\rangle\right|^{2}$ for $\tilde{A} \leftarrow \tilde{X} 0_{0}$ transition...........137

Table 4.17. Frank Condon factors $\mathrm{fv}^{\prime} \mathrm{v}^{\prime \prime}=\left|\left\langle\mathrm{v}^{\prime} \mid \mathrm{v}^{\prime \prime}\right\rangle\right|^{2}$ for $\tilde{X} \leftarrow \tilde{A} 0_{0}$ transition............137 Table 5.1 Geometric parameters, rotational constants, and $\tilde{A}-\tilde{X}$ excitation energy of the $\mathrm{CaOCH}_{2} \mathrm{CH}_{3}$ radicals .158

Table 5.2 Harmonic vibrational frequencies of $\mathrm{CaOCH}_{2} \mathrm{CH}_{3}$ in the $\tilde{X}^{2} A_{1}$ and $\tilde{A}^{2} E$ states (in cm-1). Vibrational modes are numbered following the Herzberg convention .159

Table 5.3. Relative intensities (proportional to the FC factors assuming BOA) for $\tilde{A}_{1} / \tilde{A}_{2} \leftarrow \tilde{X}(\mathrm{v}$ ’'=0) vibronic excitation transitions. The total intensity is normalized to unity .160

Table 5.4. Relative intensities (proportional to the FC factors assuming BOA) for $\tilde{A}_{1} / \tilde{A}_{2} \rightarrow \tilde{X}\left(\mathrm{v}^{\prime \prime}=0\right)$ vibronic excitation transitions. The total intensity is normalized to unity .160

Table 6.1. Geometric parameters, rotational constants, and $\tilde{A}-\tilde{X}$ excitation energy of the $\mathrm{CaOCH}_{2} \mathrm{CH}_{3}$ radicals. .170 
Table 7.1 Electronic transition frequencies and vibrational constants of different states........................................................................ 197

Table 7.2. Comparison of molecular constants wit literature reported values.........198

Table 7.3. Rotational line positions and branches for $A^{2} \Pi_{3 / 2} \leftarrow X^{2} \Sigma^{+}$transition......199

Table 7.4. Rotational line positions and branches for $A^{2} \Pi_{1 / 2} \leftarrow X^{2} \Sigma^{+}$transition......202 


\section{LIST OF FIGURES}

Figure 1.1: The LIF excitation spectrum of the $A^{2} \Sigma^{+}-X^{2} \Pi$ electronic transition of NO at a) a rotational temperature of $\sim 300 \mathrm{~K}$, and b) after supersonic cooling to a rotational temperature of $\sim 15 \mathrm{~K}$. The asterisk in a) identify $\mathrm{P}$ and $\mathrm{Q}$ branch banheads............19

Figure 1.2: Schematic representation of PESs of (a) a two-dimensional harmonic oscillator, (b) molecules with the JT interaction, e.g., methoxy $\left(\mathrm{CH}_{3} \mathrm{O}\right), \mathrm{CaCH}_{3}$ and $\mathrm{CaOCH}_{3}$, and (c and d) molecules with the PJT interaction. (c) and (d) are of different energy separations and different magnitudes of PJT interaction. For instance, (c) represents the PESs of ethoxy, while (d) represents those of isopropoxy. The spin-orbit

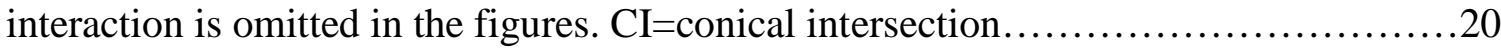

Figure 2.1: Laser ablation fixture a) schematic cross section b) solid design c) sample holder (red color indicates targeted metals) d) cross section with dimensions.

Figure 2.2: Motorized stage laser ablation nozzle fixture.

Figure 2.3: Block diagram of the electrical circuit for gear motor control...............46

Figure 2.4: Gear motor control using Arduino Uno. Insets show the details of L298 and

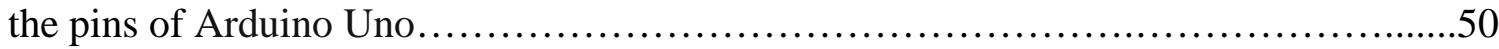

Figure 2.5: Decay process which can occur following an absorption process................51 
Figure 2.6: Schematic diagram showing the fundamental transitions involved LIF excitation spectroscopy. Red arrows indicate hot band transitions....................52

Figure 2.7: Transitions involved in laser-induced dispersed fluorescence (DF) spectroscopy. The red transition represents the laser excitation, or pump,

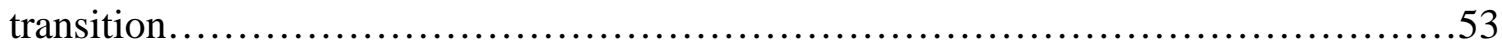

Figure 2.8: Timing aspects of experimental synchronization (not in scale).................54

Figure 2.9: schematic diagram of the LIF/DF experiment ...................................55

Figure 2.10: Principles of CRDS. Ring-down cavity is defined by two highly reflective mirrors and the sample is introduced into the cavity. Transmitted photons leaking from exiting mirror are indicated by blue arrows when the cavity is empty and by red arrows

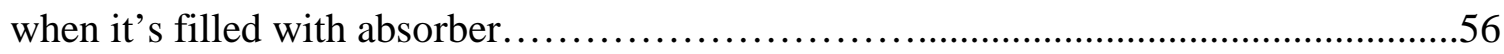

Figure 2.11: Schematic diagram of cavity ring-down spectroscopy experiment...............57

Figure 2.12: Timing aspects of CRDS experimental synchronization (not in scale)......58

Figure 3.1: The molecular orbitals of $\mathrm{CaOCH}_{3}$ included in CASSCF calculation.......103

Figure 3.2: (a and b) simulated and (c) experimental LIF spectra of the $\tilde{A}^{2} E \leftarrow \tilde{X}^{2} A_{1}$ transition of $\mathrm{CaOCH}_{3}$. Wavenumbers are relative to the origin band at $15925 \mathrm{~cm}-1$. Numbers in parentheses are relative wavenumbers in $\mathrm{cm}^{-1}$. The peaks in each doublet correspond to transitions to the $\tilde{A}^{2} E_{1 / 2}$ (lower frequency) and $\tilde{A}^{2} E_{3 / 2}$ (higher frequency) SO components......................................................... 104 
Figure 3.3: Comparison of (a) LIF and (b) CRD spectra of the origin and 41 band transitions of $\mathrm{CaOCH}_{3}$ that shows the power saturation of the origin transitions in the LIF experiment.............................................................. 105

Figure 3.4: (a and b) Simulated DF spectrum of the $\tilde{A}^{2} E \leftarrow \tilde{X}^{2} A_{1}$ origin transition of $\mathrm{CaOCH}_{3}$. DF spectra obtained by pumping the (c) $\tilde{A}^{2} E_{1 / 2} \leftarrow \tilde{X}^{2} A_{1}$ and (d) $\tilde{A}^{2} E_{3 / 2} \leftarrow$ $\tilde{X}^{2} A_{1}$ origin transitions. The numbers in parentheses are red-shift in $\mathrm{cm}-1$. The asterisked peaks in (d) are due to collision-induced $\tilde{A}^{2} E_{3 / 2} \leftarrow \tilde{X}^{2} A_{1}$ population transfer following

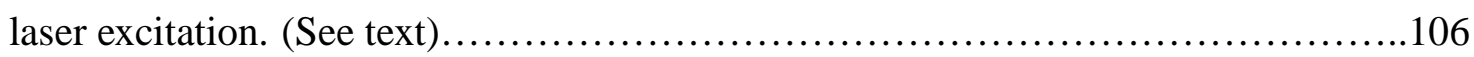

Figure 3.5: DF spectra obtained by pumping different vibronic bands of the $\tilde{A}^{2} E \leftarrow$ $\tilde{X}^{2} A_{1}$ transition. The asterisked peaks are due to collision-induced $\tilde{A}^{2} E_{3 / 2} \leftarrow \tilde{A}^{2} E_{1 / 2}$ population transfer following laser excitation. "X" and " $\mathrm{Y}$ " denote unidentified

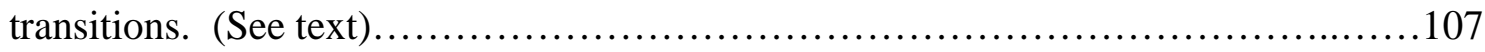

Figure 3.6: DF spectra obtained by pumping different vibronic bands of the $\tilde{A}^{2} E \leftarrow$ $\tilde{X}^{2} A_{1}$ transition. Spectra of bands other than origin band in the LIF spectrum are blueshifted. "X" and "Y" denote unidentified transitions. (See text)

Figure 3.7: Qualitative energy level scheme of the spin-vibronic energy levels of the $\tilde{A}^{2} E$ state of $\mathrm{CaOCH}_{3}$ and $\tilde{A}^{2} E \leftarrow \tilde{X}^{2} A_{1}$ vibronic transitions. (a) in the absence of JT and SO interactions. (b) With the linear JT effect included. (c) With both linear and quadratic JT effect included. (d) With both JT and SO interaction included

Figure 5.1: The molecular orbitals of $\mathrm{CaOCH}_{2} \mathrm{H}_{3}$ included in CASSCF calculation...152 
Figure 5.2: (a) simulated by state averaged CASSCF method (b and c) simulated for the $\tilde{A}_{1}^{2} A^{\prime} \leftarrow \tilde{X}^{2} A^{\prime}$ and $\tilde{A}_{2}^{2} A^{\prime \prime} \leftarrow \tilde{X}^{2} A^{\prime}$ transition of Ca-ethoxide. Wavenumbers are relative to the origin band at $15882 \mathrm{~cm}^{-1}$. Numbers in parentheses are relative wavenumbers in $\mathrm{cm}$ 1. .153

Figure 5.3: (a) simulated by state average CASSCF method. (b and d) simulated for the $\tilde{A}_{1}^{2} A^{\prime} \rightarrow \tilde{X}^{2} A^{\prime}$ and $\tilde{A}_{2}^{2} A^{\prime \prime} \rightarrow \tilde{X}^{2} A^{\prime}$ transition of Ca-ethoxide. DF spectra by pumping the (c) $\tilde{A}_{1}^{2} A^{\prime} \leftarrow \tilde{X}^{2} A^{\prime}$ and (e) $\tilde{A}_{2}^{2} A^{\prime \prime} \leftarrow \tilde{X}^{2} A^{\prime}$ origin transitions. Numbers in the parentheses are red-shift in $\mathrm{cm}^{-1}$. The asterisked peaks in (d) are due to collision-induced population transfer .154

Figure 5.4: DF spectra by pumping different vibronic bands of the $\tilde{A}_{1} / \tilde{A}_{2} \leftarrow \tilde{X}$ transitions of ca-ethoxide. Numbers in the parentheses are red-shift in $\mathrm{cm}^{-1}$. " $\mathrm{X}$ ", "Y" and “Z” denotes unidentified transitions. 155

Figure 5.5: DF spectra by pumping different vibronic bands of the $\tilde{A}_{1} / \tilde{A}_{2} \leftarrow \tilde{X}$ transitions of ca-ethoxide. Numbers in the parentheses are red-shift in $\mathrm{cm}^{-1}$. Spectra of bands other than origin band in the LIF spectrum are blue-shifted. 156

Figure 5.6: Comparison of (a) LIF and (b) CRD spectra of the origin and band transitions of $\mathrm{CaOCH}_{3} \mathrm{CH}_{2}$ that shows the power saturation of the origin transitions in the LIF experiment .157

Figure 6.1: a and b is simulated for the $\tilde{A}_{1}^{2} A^{\prime} \leftarrow \tilde{X}^{2} A^{\prime}$ and $\tilde{A}_{2}^{2} A^{\prime \prime} \leftarrow \tilde{X}^{2} A^{\prime}$ transition of Ca-isopropoxide. Wavenumbers are relative to the origin band at $15844 \mathrm{~cm}^{-1}$. Numbers in parentheses are relative wavenumbers in $\mathrm{cm}^{-1}$. 173 
Figure 6.2: (a and b) Simulated for the $\tilde{A}_{1}^{2} A^{\prime} \rightarrow \tilde{X}^{2} A^{\prime}$ and $\tilde{A}_{2}^{2} A^{\prime \prime} \rightarrow \tilde{X}^{2} A^{\prime}$ transition of Ca-isopropoxide. DF spectra by pumping the (c) $\tilde{A}_{1}^{2} A^{\prime} \leftarrow \tilde{X}^{2} A^{\prime}$ and (d) $\tilde{A}_{2}^{2} A^{\prime \prime} \leftarrow \tilde{X}^{2} A^{\prime}$ origin transitions. Numbers in the parentheses are red-shift in $\mathrm{cm}^{-1}$. The asterisked peaks in (d) are due to collision-induced population transfer. .172

Figure 6.3: DF spectra by pumping different vibronic bands of the $\tilde{A}_{1} / \tilde{A}_{2} \leftarrow \tilde{X}$ transitions of ca-isopropoxide. Numbers in the parentheses are red-shift in cm-1. " $\mathrm{X}$ " and "Y" denotes unidentified transitions. 173

Figure 6.4: DF spectra by pumping different vibronic bands of the $\tilde{A}_{1} / \tilde{A}_{2} \leftarrow \tilde{X}$ transitions of ca-isopropoxide. Numbers in the parentheses are red-shift in cm-1. Spectra of bands other than origin band in the LIF spectrum are blueshifted. .174

Figure 6.5: Comparison of (a) LIF and (b) CRD spectra of the origin and 151 band transitions of $\mathrm{CaOCH}\left(\mathrm{CH}_{3}\right)_{2}$ that shows the power saturation of the origin transitions in the LIF experiment. 175

Figure 7.1: Schematic diagram of the pulsed slit jet nozzle

Figure 7.2: Electronic structure of YO. Solid arrows indicate cooling and re-pump laser transitions. Dashed arrows indicate decay paths.

Figure 7.3: CRD spectra of $A^{2} \Pi_{3 / 2} \leftarrow X^{2} \Sigma^{+}$ .193

Figure 7.4: CRD spectra of $A^{2} \Pi_{1 / 2} \leftarrow X^{2} \Sigma^{+}$

Figure 7.5: CRD spectra of $A^{2} \Pi_{3 / 2} \leftarrow X^{2} \Sigma^{+}$ 195 
Figure 7.6: Schematic diagram of Two beam experiments. M: ring-down mirror, S1 : the intensity of fixed frequency transition, S2 : Intensities of the transitions of scanning frequencies, I: normalized frequency......................................196

Figure 8.1 (a) Principle of SEP and SEP-CRU spectroscopy techniques. (b) Simplified experimental setups of SEP-CRU spectroscopy. When the pump and dump laser wavelengths are well separated, dichroic mirrors or (polarization) beam splitters are used to overlap them (top). When the wavelengths are close, a " $\mathrm{X}$ "-shaped two-wavelength scheme is adopted (bottom). "M" =cavity ring-down mirror. "W" =window. PMT=photomultiplier tube. The spectrograph is used to select the wavelength window for fluorescence detection and to filter out laser scattering. (c) Ring-down curves without $\left(\tau_{0}\right)$ and with $(\tau)$ resonance between the dump laser frequency and an $\tilde{A}^{2} \Pi \rightarrow \tilde{X}^{2} \Sigma^{+}$ transition. $c$ is the speed of light. $R$ is the reflectivity of ring-down mirrors. $\sigma_{\mathrm{a}}$ and $\sigma_{\mathrm{e}}$ are cross sections for absorption and emission, respective. $N_{1}$ and $N_{2}$ are populations of the ground and excited states, respectively. (d) jet-cooled CRD spectrum of the $\tilde{A}^{2} \Pi \leftarrow \tilde{X}^{2} \Sigma^{+}$ origin band of YO compared with simulation.................................213 


\section{CHAPTER I}

\section{INTRODUCTION}

Almost no chemical reactions or very few chemical reactions proceed directly from reactants to products. In most of the cases, a reaction occurs via several elementary steps, each involving atomic or molecular chemical intermediates that are produced and consumed very rapidly during each stage of the reaction. In general, the reaction must be initiated by 'activating' one, or more of the bonds in the reactant and this may be achieved using heat or light or using catalysis to lower the activation energy of a key step. The chemical intermediates of any reactions cannot be easily isolated unlike the starting reactants or the final products.

Chemical intermediates i.e. free radicals play a crucial role in the outcome of many reactions and processes in all areas of chemistry, ranging from biochemical events and catalysis, through to combustion, atmospheric and even interstellar processes. Detection and physical characterization of reaction intermediates inevitably lead to a complete understanding of the intrinsic role in many chemical pathways. However, due to the short lifetime and instability i.e. high reactivity of intermediates in most chemical reactions, their absolute concentrations are usually low. 
Anciently, routine laboratory instruments, such as standard IR or UV/VIS spectrometers, have proved too insensitive to detect the low radical concentrations in chemical reactions mainly limited by the resolution and sensitivity of the techniques. In the last two decades, more specialized spectroscopic techniques, often involving light sources as laser, have made the study of free radicals much easier and are primarily responsible for the increased interest in these species, especially in the gas-phase. Laserinduced fluorescence (LIF) and Dispersed-fluorescence spectroscopy (DF) ${ }^{8}$ are the most widely used methods for detecting radicals, although it does suffer from significant limitations. Other highly sensitive laser-based spectroscopic techniques are also important, examples of which include resonance-enhanced multi-photon ionization (REMPI), ${ }^{9}$ diode laser spectroscopy ${ }^{10}$ and time of flight mass spectrometry (TOF). Most recently one of the highly sensitive absorption-based spectroscopic methods named Cavity Ring-Down Spectroscopy (CRDS) became very famous in detecting free radicals. Additionally, these techniques are often used to observe radicals that can be seeded, at low concentrations, into inert gas supersonic jets. Supersonic jets ${ }^{11}$ provides favorable environments for studying short-lived chemical species by creating very low vibrational and rotational temperature of the species.

In the supersonic jet, molecules will undergo a very little number of collisions. Those collisions take place to the early stages of the expansion, occur with inert gas atoms and these have the effect of cooling the internal degrees of freedom of the molecules. The consequence of this effect is that spectra are considerably simplified by minimizing the number of electronic transitions in comparison with those recorded at room temperature since fewer molecular energy levels have significant populations. For 
larger molecules, which may have very complex rotational and vibrational energy level manifolds, the simplification brought about by supersonic jet cooling is often a precious aid in spectroscopic analysis. A complete detailed discussion of supersonic jet expansion will be given in Chapter 2 . 


\subsection{Metal containing free radicals}

The history of free radial studies is significant and interesting. Historically, free radicals were generated by using Broida type oven, pyrolysis, electric discharge and using laser photolysis. The following discussion will concentrate on the development of techniques for studying and characterizing of metal-containing free radicals since these are the target in the current work. The interaction of metals with organic or inorganic molecules has significant importance in many areas of chemistry, with perhaps the most eminent areas being catalysis and synthetic organometallic chemistry. Many catalytic processes involve cleavage of a carbon-carbon sigma bond, via infusion of a transition metal directly into the bond.

Another extremely important family of reagents, that are the most versatile in organic chemistry, are Grignard reagents (R-Mg-X, where $\mathrm{R}=$ alkyl and $\mathrm{X}=$ halide) ${ }^{12}$ Very often, the specific mechanisms of magnesium-carbon bond formation in reactions involving Grignard reagents are imprecise. Clearly, identifying molecules that are likely to be involved in such mechanisms is important with respect to improving the efficiency of many organic syntheses which lead to the production of numerous types of new molecules. The interaction of metal atoms with organic ligands has important implications in biochemistry as well. Many key biological functions include the formation and breaking of metal-carbon bonds. For example, the role of metals, such as iron and magnesium, in hematology, plant photosynthesis ${ }^{12}$ or polymerization ${ }^{13}$.

Another major area where metal-ligand interactions are crucial is in the semiconductor industry. The optimum growth conditions such as temperature and partial 
pressures of the precursors for preparing semiconductor materials are indispensable. Gasphase studies of the product decompositions and mechanisms of various organometallic semiconductor precursors $\left(\left(\mathrm{CH}_{3} \mathrm{CH}_{2}\right)_{3} \mathrm{Ga}, \quad\left(\mathrm{CH}_{3}\right)_{6} \mathrm{Si}_{2}\right.$, etc. $)$ should result in more competent methods for semiconductor preparation. ${ }^{14}$ One of the keys to understanding these processes is to determine the nature of the metal-ligand interactions, and in particular to characterize the intermediates formed on decomposition and the manner by which the metal influences the dynamics and kinetics of the decomposition process.

Other areas of chemistry which involve important contributions from metalcontaining free radicals include combustion, atmospheric and interstellar chemistry. Initially, the combustion chemistry of metals got attention because of the intense colors that are observed when metals or metal salts are burned in flames. The mechanistic aspects of combustion chemistry involving metals are still relatively unknown since they are thought to embroil highly complex radical reactions of metal monoxides and hydroxides. ${ }^{15}$ Most recent studies of metal combustion have leaned towards elucidating the dynamical and kinetic aspects of these processes. ${ }^{16}$ Metal containing radicals are thought to play crucial roles in the complex chemistry of Earth's upper atmosphere. The mostly known metals in this regard are iron, magnesium, and sodium. The upper atmosphere chemistry of sodium metal is particularly interesting. Sodium, and many of its compounds, are highly reactive and undergo many interactions, including those that are responsible for the sodium airglow.

The basic mechanism for the sodium airglow (caused by emission from electronically excited sodium atoms ( $\mathrm{Na}^{2} \mathrm{P}_{-}^{2} \mathrm{~S}$ transition), was first suggested by Chapman, in $1939,{ }^{17}$ as, 


$$
\begin{aligned}
& \mathrm{Na}+\mathrm{O}_{3} \rightarrow \mathrm{NaO}+\mathrm{O}_{2} \\
& \mathrm{NaO}+\mathrm{O} \rightarrow \mathrm{Na}+\mathrm{O}_{2}
\end{aligned}
$$

Although this may look like a relatively simple process, the specifics of the mechanism are still uncertain. The two lowest electronic states of sodium oxide $\left(\mathrm{NaO}, \mathrm{Na}^{2} \mathrm{P}^{-}{ }^{2} \mathrm{~S}\right)$ are very close together and both are energetically accessible in reaction (1.1). It is thought that the specific electronic state of the $\mathrm{NaO}$ formed in reaction (1.1) has significant consequences upon the sodium $(\mathrm{N})$ formed in reaction (1.2). Laboratory-based studies involving the characterization of the electronic states of $\mathrm{NaO}$ should ultimately be useful in identifying the precise role of $\mathrm{NaO}$ in the Chapman mechanism.

Alkaline-earth metal-containing free radicals are also thought to be involved in both atmospheric and interstellar chemistry. It has been hypothesized that the ablation of metals from meteors results in the formation of $\mathrm{CaOH}$ in the upper atmosphere. ${ }^{18}$ Additionally, a number of alkaline-earth metal-containing molecules should be present in the atmospheres of cool-oxygen rich stars. ${ }^{19}$ Other metal-containing molecules, notably magnesium containing molecules such as $\mathrm{MgNC}^{20}$ and $\mathrm{MgCN},{ }^{21}$ have been identified in the circumstellar envelope of the IRC+10216 carbon star. It is probably only a matter of time before many other metal-containing molecules are detected in stellar and interstellar space. 


\subsection{Early studies of metal-containing free radicals}

At the beginning of the 1980's, most of the spectroscopic information of metal-containing species involved diatomic molecules. Indeed, all the available experimental and theoretical information about metal-containing molecules obtained before 1977 was collected together by Huber and Herzberg and published in their book, entitled 'Molecular spectra and molecular structure IV: Constants of diatomic molecules'.22 This contains complementary data for all diatomic, not only metalcontaining, derived from a wide variety of spectroscopic methods. Early production of metal-containing diatomic molecules had been carried out via methods such as flash photolysis, ${ }^{23}$ shock tubes, ${ }^{24}$ exploding wires, ${ }^{25}$ arcs, ${ }^{26}$ and in oxygen-hydrocarbon flames seeded with the appropriate metal compounds. Spectra of molecules formed in these sources were often extremely congested due to the high internal rotational temperatures of the molecules. Examples of spectroscopic investigations of polyatomic metalcontaining radicals were rare in the literature until the early 1980's. The first polyatomic alkaline-earth metal derivatives were studied by Herschel as far back as $1823,{ }^{27}$ when the monohydroxides were prepared in a flame environment and studied by emission spectroscopy. Significant flame emission studies of monohydroxides were carried out by Charton and Gaydon, amongst others, in the 1950's and 60's. They observed emission of many metal-containing oxides and hydroxides, including $\mathrm{BaOH}, \mathrm{SrOH}, \mathrm{SnOH}, \mathrm{MnOH}$ and $\mathrm{Al}_{2} \mathrm{O}_{3}$. The inauguration of the Broida oven in $1975^{28}$ provided a simple method of preparing metal-containing diatomics, such as $\mathrm{BaO}, \mathrm{BaF}$, and $\mathrm{FeO}$, with significantly lower internal temperatures $(-500 \mathrm{~K})$ than had been obtainable with previous production methods. Therefore, the quality of spectra was considerably enhanced in many cases 
using this technique. In a Broida oven, metal atoms are evaporated in a small ceramic crucible which is resistively heated using a tungsten wire basket. The vaporized metal atoms are entrained into a flowing inert carrier gas, such as argon or helium, which passes around the crucible. Products are formed by mixing the metal vapor with an appropriate oxidizing agent, a short distance above the crucible.

A critical advantage of the Broida oven is the large number of product molecules $\left(10^{13}\right.$ molecules $\left.\mathrm{cm}^{-3}\right)$ that can be entrained into the carrier gas flow. ${ }^{29}$ Also, as a significant number of product molecules are formed in electronically excited states, the method is ideally suited to optical emission spectroscopies. A chemiluminescent flame is often observed above the mixing region. However, although the technique works pretty well for the metals which have a significant vapor pressure at temperatures below -1000 $\mathrm{K}$, the thermal insulation requirements of the Broida oven limit its use for metals with higher melting points. This is why, although investigations of many metal-containing diatomics were carried out using this method in the late 1970's and early 1980's, they were mainly limited to molecules involving the alkali and alkaline-earth metals.

${ }^{30} \mathrm{~A}$ new method for producing metal-containing diatomics, and simple polyatomic, of refractory metals, was invented by Trkula and Harris in $1982 .{ }^{31}$ The technique is based upon the well-established method of hollow-cathode sputtering to produce metal atoms in the gas-phase. ${ }^{32}$ However, the apparatus designed by Trkula and Harris efficiently mixes metal atoms into a flow of inert carrier gas by passing it through the hollow cathode. The metal atoms are carried into a mixing region where an appropriate gas is injected to produce the metal-containing molecules. 
In a similar way to the Broida oven, a chemiluminescent flame is usually formed which can be probed via optical emission spectroscopy. The product molecules are produced at ambient rotational temperatures, ranging from 300-400 K. Examples of molecules produced via this method include $\mathrm{CuO}, \mathrm{CuH}$ and $\mathrm{CuOH} .{ }^{33}$ Indeed, this method is still used regularly to produce refractory metal-containing molecules, examples include AgO, FeH, TiH, and BeH.

${ }^{34,}{ }^{35}$ Another flow-type method for generating metal-containing diatomics has been employed by several research groups and incorporates a combination of resistive heating and microwave discharge or electron bombardment. A solid metal-containing salt is resistively heated in a crucible to create gas-phase molecules. The molecules are entrained into a flow of inert carrier gas, containing a gaseous precursor/reactant molecule. The gas mixture is then subjected to a microwave discharge, or electron bombardment, resulting in the formation of the desired radical among many other possible products. Again, this method is specifically suited to optical emission spectroscopy since the species are frequently produced in electronically excited states. A large number of, predominantly, diatomic metal-containing molecules have been produced by this method, including $\mathrm{TiO}, \mathrm{SrF}$, ScO and $\mathrm{CoO} .{ }^{36}, 37$ For example, $\mathrm{TiO}$ was produced via resistive heating of $\operatorname{Ti}\left(\mathrm{C}_{5} \mathrm{H}_{5}\right)_{2} \mathrm{Cl}_{2}$ in a tantalum furnace prior to microwave discharge in the presence of oxygen gas and argon. 


\subsection{Molecular beam and supersonic jet expansions}

The obvious solution to the problems caused by relatively high temperatures is to generate molecules in the ultra-cold environment of a supersonic jet. One example of supersonic cooling over of molecules over high temperature is given in figure-1.1 ${ }^{38}$. The use of continuous and pulsed supersonic jet expansions to aid rotational and vibrational simplification of gas-phase spectra (see Chapter 2) has been recognized since the late 1970 's. Examples of the radical generation immediately prior to, or during the supersonic expansion, are commonplace in the literature. However, until the early 1980's, most studied radicals involved inorganic, or organic, radicals that were produced by fragmentation of larger parent molecules. The conventional methods of radical production fragments included seeding of a suitable precursor into an inert carrier gas,

followed by laser photolysis at the throat of the nozzle, ${ }^{39}$ electrical discharge prior to or during the expansion, ${ }^{40}$ or pyrolysis before the expansion ${ }^{41}$.

Unfortunately, these methods are rarely appropriate for making metalcontaining species because of the dearth of suitable precursors. An important criterion of suitable precursor must not only possess a structure such that fragmentation of one, or more, bonds will yield the desired product, but it must also be reasonably volatile in order to obtain gas-phase species. At present, a number of metal-containing polyatomic which have been produced prior to, or during supersonic expansion by laser photolysis, electrical discharge or pyrolysis of a metal-containing precursor, are quite rare. Exceptions include experiments carried out by the Miller and Ellis research groups in the early and mid-1990s, involving the $\mathrm{ZnCH}_{3}, \mathrm{ZnC}_{2} \mathrm{H}_{5}$, and $\mathrm{CdCH}_{3}$ free radicals. 
Supersonically cooled $\mathrm{ZnCH}_{3}{ }^{42}$ and $\mathrm{ZnC}_{2} \mathrm{H}_{5}{ }^{43}$ were both successfully generated and studies by Ellis and co-workers following electrical discharge of the appropriate gasphase dialkyl-zinc precursor immediately prior to supersonic expansion. In a series of separate investigations by Miller and co-workers $\mathrm{ZnCH}_{3}, \mathrm{CdCH}_{3}$ and their inert gas complexes were prepared by laser photolysis of the respective dimethyl-metal precursors just after the supersonic nozzle ${ }^{44}$.

In order to generate a wider range of supersonically cooled metal-containing molecules, an alternative production method was required. In 1981, a seminal article by Smalley and coworkers described a new technical method for creating small metallic clusters by laser ablation of a metal target prior to supersonic expansion ${ }^{45}$. Throughout the 1980's a significant number of articles were published by Smalley and coworkers involving the spectroscopy of many neutral, and ionic, small metal clusters, including $\mathrm{Cu}_{2}, \mathrm{Cr}_{2}, \mathrm{Mo}_{2}, \mathrm{Cu}_{3}, \mathrm{~V}_{2}, \mathrm{Ni}_{2}, \mathrm{Si}$ ”, Al” and GaAs. Bondybey and coworkers were also contributed to a wealth of information throughout the 1980's regarding gas-phase and matrix isolation studies of small metallic clusters prepared using the Smalley ablation nozzle $^{46}$. In 1990, the first examples involving the combination of supersonic cooling in jet with laser ablation to produce non-cluster metal-containing polyatomic radicals were reported by Whitham et al. ${ }^{47}$.These workers obtained LIF spectra of CaCCH, CaNC, and $\mathrm{CaNH}_{2}$ by this route. Whitham et al. proposed that calcium-containing molecules were created by insertion/elimination reactions of electronically excited calcium atoms formed by laser ablation with small organic and inorganic precursors, entrained into a pulse of helium or argon, 10-15 mm behind a supersonic nozzle. Laser ablation was carried out using the second harmonic of an Nd: YAG laser, operating at $532 \mathrm{~nm}$. The potentiality of 
laser ablation as a source of a distinct range of metal-containing molecules in the gasphase cannot be understated. All metals, no matter how refractory, can be ablated with an intense laser pulse. Indeed, since the first example of its use in 1981, a significant number of spectroscopic studies of supersonically cooled metal-containing radicals have been carried out using laser ablation in combination with supersonic expansion.

The Steimle group has produced many diatomic and polyatomic molecules, including alkaline earth containing species, such as $\mathrm{CaOCH}_{3}$ and $\mathrm{CaNH}_{2}$, through to transition metal molecules, such as $\mathrm{YC}_{2}$ and $\mathrm{MoN}^{48}$. Other groups have also been successful. The groups of Simard and Hackett, based in Ottawa, published numerous numbers of research articles involving metal-containing radicals prepared via laser ablation/photolysis, such as YCCH, LaF, and $\mathrm{NbS}^{49,50}$. The research groups of Benath, Miller and Ellis have also published a vast number of articles describing the spectroscopy of supersonically cooled metal-containing molecules prepared by laser ablation/photolysis. Some of these include $\mathrm{SrOH}, \mathrm{LiO}, \mathrm{MgCCH}, \mathrm{CaC}_{5} \mathrm{H}_{5}, \mathrm{MgCH}_{3}, \mathrm{Al}_{2} \mathrm{O}$ and $\mathrm{CaNH}_{2}{ }^{51,52}$. To date, a group of metal-containing molecules that have not received much attention are polyatomic molecules containing d-block metals. Due to the high density of electronic states, extreme congestion can lead to significant perturbations in the spectra of these molecules. Consequently, assignments of the full band system can be extremely difficult to achieve. Laser ablation does have drawbacks since it is usually combined with various forms of laser spectroscopy; multiple laser systems are required, including the ablation laser and one, or more, probe lasers. Consequently, the initial cost of setting up a laboratory is rather high. Another drawback is that the ablation process does not usually produce a clean source of metal-containing molecules. For example, 
experiments in the Ellis laboratory have shown that laser ablation of calcium metal in the presence of acetone produces a number of metal-containing radicals including $\mathrm{CaCCH}$, $\mathrm{CaCH}_{3}, \mathrm{CaH}, \mathrm{Ca}_{2}$ and $\mathrm{CaOH}$, in addition to other products such as $\mathrm{C}_{3}, \mathrm{CH}$ and $\mathrm{OH}$. This can be a problem when using probe techniques such as laser-induced fluorescence spectroscopy, since all the above molecules does fluoresce and have strong LIF spectra.

\subsection{This thesis}

Highly reactive and unstable molecules, such as ions and free radicals, are known to be important species in many chemical reactions. As a result, there is a great interest in characterizing and understanding the properties of these molecules. However, due to the transient nature of these species, the experimental conditions required for their study is extremely challenging, often requiring the use of sensitive spectroscopic techniques such as laser-induced fluorescence (LIF) and cavity ring-down spectroscopy (CRDS). Most of these studies have been on small- or medium-sized organic or inorganic species. There has been relatively less work on metal-containing free radicals. Metalcontaining free radicals are important intermediates in interactions between metals and organic molecules. For instance, Interaction between ethylene and a titanium metal center, for example, is involved in the Ziegler-Natta polymerization of ethylene ${ }^{1}$.

These free radicals are of special interest to theoretical molecular spectroscopists too. Many such molecules, including the organometallic monomethyl radicals such as $\mathrm{MgCH}_{3}, \mathrm{CaCH}_{3}$, and $\mathrm{SrCH}_{3}$ as well as their corresponding methoxide radicals $\left(\mathrm{MgOCH}_{3}, \mathrm{CaOCH}_{3}\right.$ and $\left.\mathrm{SrOCH}_{3}\right)$ have $\mathrm{C}_{3 \mathrm{v}}$ symmetry and their first 
electronically excited $\left(\tilde{A}^{2} E\right)$ state would be orbitally degenerate if there were no spinvibronic interactions. The $\tilde{A}^{2} E$ state, however, is subject to spin-orbit (SO) and JahnTeller (JT) interactions, both of which remove the degeneracy. Its potential energy surface (PES) is distorted by the JT interaction and further split by the SO interaction into two components, $E_{1 / 2}$ and $E_{3 / 2}$. Because both interactions compete for the orbital angular momentum, the $\mathrm{SO}$ splitting between the $\mathrm{E}_{1 / 2}$ and $\mathrm{E}_{3 / 2}$ states is quenched by the JT effect, and vice versa.

Potential energy surface (PES) is one of the most important concepts in quantum chemistry and chemical kinetics. In quantum chemical calculations, the geometry optimization of a molecule is done by finding the minima on the PES. Given a PES, the energy levels of a molecule can in principle be determined by solving the Schrödinger equation. In chemical reactions, reactants usually have to overcome some barriers on the PES to form products. Figure 1.2a shows the PES of a two-dimensional harmonic oscillator, which is a good model for many molecules.

The concept of PES is based on the Born-Oppenheimer (BO) approximation, ${ }^{53}$ which states that because of the large difference in the masses of electrons and nuclei, their motion can be treated separately. Although the BO approximation is usually valid in the studies of molecular dynamics and chemical kinetics, there are many cases in which it breaks down and the coupling between electronic and nuclear motion becomes significant ${ }^{54,55}$. One important example of this breakdown occurs when two or more PESs are of the same energy, or degenerate, due to symmetry and another when the PESs lie very close in energy i.e., they are semidegenerate. In the former case, the orbital degeneracy is removed by the Jahn-Teller (JT) 
interaction, and the two PESs form the "Mexican hat" potential (Fig. 1.2b). In the latter case, the "Mexican hat" is further distorted by the pseudo-Jahn-Teller (PJT) interaction, and the PES becomes even more complicated (Figs. 1.2c and 1.2d). In both cases, the nonadiabatic vibronic (vibrational-electronic) interaction distorts the geometry of the molecule and lowers its total energy. As a result, the two PESs meets at one or more conical intersections (CIs), singularities on the PESs, indicated in Figs. 1.2b-d.

An effective approach to study the PJT interaction is by asymmetric isotope or methyl substitution of JT molecules. The asymmetric isotope substitution, such as deuteration, lowers the vibrational symmetry due to mass effect but the electronic symmetry, i.e., the symmetry of the PESs remains unchanged. To quantitatively understand the PJT interaction between two close-lying states in molecules with low symmetry, a systematic spectroscopic investigation of several groups of free radicals can be used. The LIF study on the methyl-substituted $\mathrm{CaCH}_{3}$ and $\mathrm{CaOCH}_{3}$ radicals provide more information on the similarity and difference between single and double methyl substitutions and different orders of contributions of the PJT effect to the molecular constants.

The study of the gas phase organometallic intermediates has been carried out mostly using a Broida type oven to generate these species in the gas phase. The Broida oven method uses a gas-phase synthesis approach involving metal vaporization in a crucible. Due to the heat produced by the furnace, the molecules have high internal temperatures and so that the transitions are highly congested and broadened due to Doppler and collision broadening. The observed spectra, therefore, contain only broad vibronic features and are very difficult to analyze. In addition, population transfer after 
laser excitation is facilitated by the high temperature, leading to extra peaks in the spectra.

An alternative to the Broida oven method is laser ablation. This technique can be easily combined with a supersonic jet expansion to produce a cold source of metal atoms. Laser ablation has proved to be an extremely successful in-situ method of producing gas-phase intermediates. A wide variety of molecules, including metal clusters, van der Waals complexes, and many organic and inorganic metal-containing intermediates, have been prepared using this method. An experimental apparatus for laser-induced fluorescence (LIF)/dispersed fluorescence (DF) spectroscopy under supersonic jet expansion conditions has been constructed. Laser ablation or dischargeassisted laser ablation has been used to generate free radicals. Vibrational structures of the ground and excited electronic states reveal themselves in the DF and LIF spectra, respectively.

Quantum chemical calculations have been carried out to assist the identification of the spectral carriers and simulation of experimentally obtained spectra, from which molecular parameters such as electronic transition energies and vibrational frequencies of both electronic states can be determined. These parameters in return can be used to benchmark the calculations. Many free radicals cannot be studied by the LIF/DF technique due to low fluorescence quantum yield and/or an extremely short lifetime. Even for small systems, the vibronic band intensities are often contaminated by intramolecular relaxation dynamics, in such cases, these techniques cannot be used for reliable intensity measurements. For instance, both predissociation and intersystem crossing (ISC) in an excited electronic state lead to fluorescence quenching. In those 
cases, a direct absorption technique can be used to measure the electronic spectra of jetcooled molecules. Another advantage of using cavity ring-down spectroscopy is the accurate measurement of the Franck-Condon (FC) factor. Fluorescence spectroscopy is a two-step process. In this method, it is very easy to saturate the transition using high photon flux which excites the excess population. But using CRDS as the intra-cavity photon is energy is very low, so the signal intensity will be quite accurate and much reliable than LIF.

In our lab cavity ring-down spectroscopy (CRDS) is used for investigation of molecules and free radicals. The method is based on the measurement of the time constant of decay of a pulse of light trapped in a high finesse optical cavity made of two high-reflectivity mirrors. CRDS is essentially a multipass absorption spectroscopic technique useful for observing relatively weak transitions. The extremely high sensitivity (sub-ppm) of the CRDS technique makes it particularly suitable for investigation of reaction intermediates in jet expansion, which usually are of low concentration. Furthermore, because most molecules have absorptions due to electronic transitions in the $\mathrm{UV} /$ visible region even when the excited state is radiationless, the absorption-based CRDS technique is more versatile than LIF/DF technique. In the current research work CRDS have been used to to compensate the power saturation of the observed transition intensities in LIF experiments. The CRDS along with LIF experiment and the vibronic analysis of the molecules will reveal the eligibility of laser cooling and also electron elctro-dynamic measurement of finding new physics. 

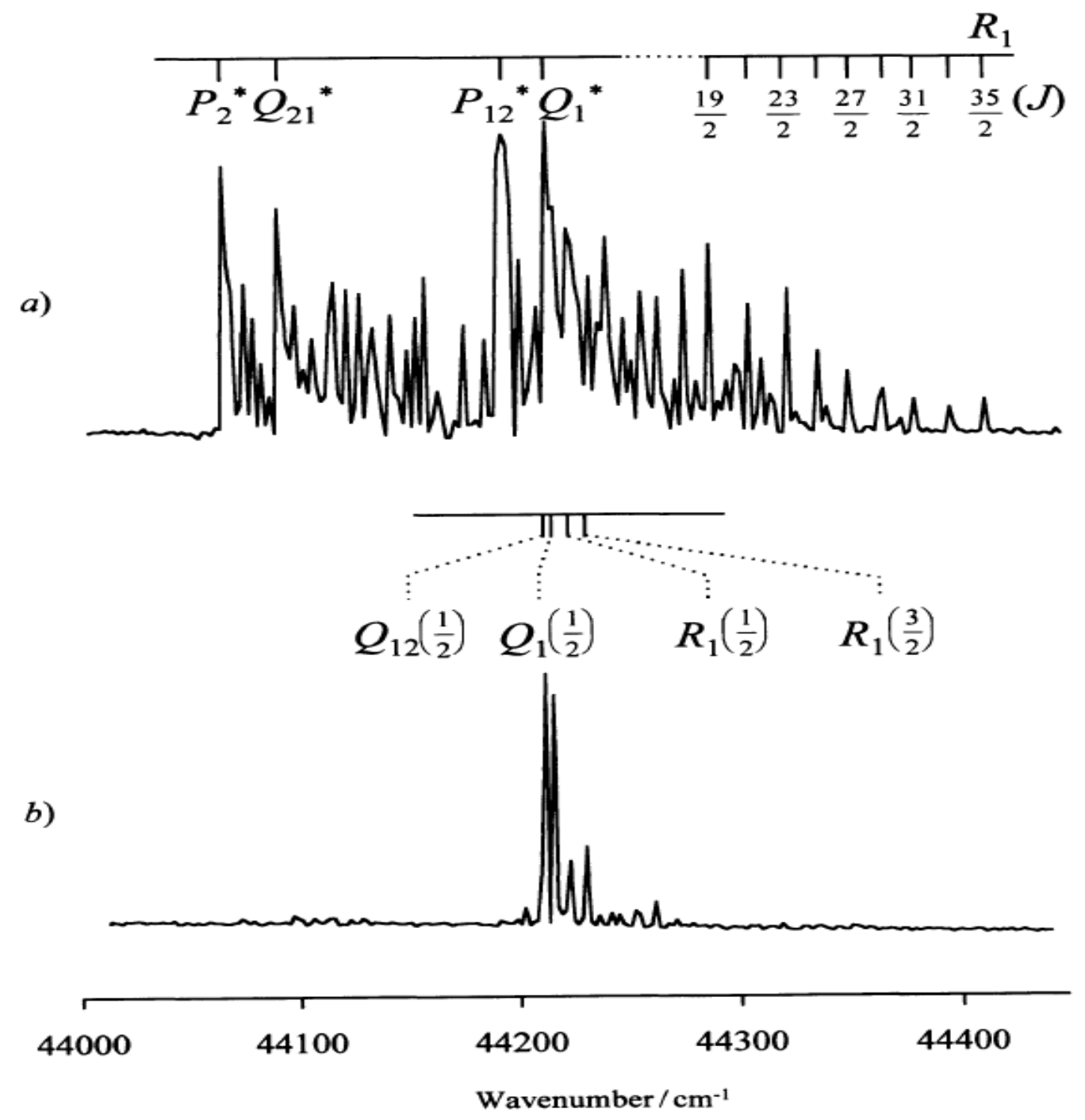

Figure 1.1: The LIF excitation spectrum of the $A^{2} \Sigma^{+}-X^{2} \Pi$ electronic transition of NO at a) a rotational temperature of $\sim 300 \mathrm{~K}$, and b) after supersonic cooling to a rotational temperature of $\sim 15 \mathrm{~K}$. The asterisk in a) identify $\mathrm{P}$ and $\mathrm{Q}$ branch banheads. 

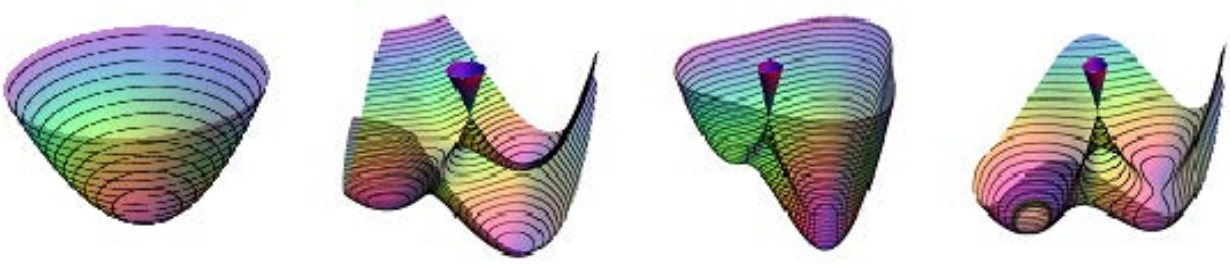

Figure 1.2: Schematic representation of PESs of (a) a two-dimensional harmonic oscillator, (b) molecules with the JT interaction, e.g., methoxy $\left(\mathrm{CH}_{3} \mathrm{O}\right), \mathrm{CaCH}_{3}$ and $\mathrm{CaOCH}_{3}$, and (c and d) molecules with the PJT interaction. (c) and (d) are of different energy separations and different magnitudes of PJT interaction. For instance, (c) represents the PESs of ethoxy, while (d) represents those of isopropoxy. The spin-orbit interaction is omitted in the figures. $\mathrm{CI}=$ conical intersection. 


\section{CHAPTER II}

\section{EXPERIMENTAL}

The chemical intermediates studied in research work for this dissertation were prepared under supersonic jet conditions using a pulsed laser ablation/photolysis technique. The applied experimental methodologies can be divided into two categories: fluorescence and direct absorption spectroscopy. For the fluorescence experiment, two types of pulsed laser-based spectroscopy were used to record electronic spectra, namely laser-induced fluorescence (LIF) for excited state vibronic structure investigations, dispersed fluorescence (DF) spectroscopy for the ground state vibronic structure investigations. For the direct absorption measurement, a highly sensitive technique, named cavity ring-down spectroscopy (CRDS) have been used. LIF/DF is used to study the electronic transition of metal-containing alkoxide radicals by measuring the fluorescence of visible excitation. CRDS is used in this research for two major purposes I) accurate measurement of the intensities of the metal-containing radicals that were studied using LIF, II) Electronic transitions of yttrium oxide (YO) in the visible region. The two methodologies will be discussed separately in this chapter. 


\subsection{Radical production}

All the laser ablation/photolysis experiments performed in this work were carried out under high vacuum conditions. The principal incentive for using such conditions arose from the physical requirements for attaining supersonic cooling (see section 2.2.3). However, a vacuum was also required in order to minimize the reaction of the radical species with residual background gas molecules during the laser ablation process. The experimental apparatus of LIF/DF consists of a vacuum chamber, gas delivery system, detection system, and laser system. Radical production in the LIF experiment worth explaining each of the components of the experimental apparatus separately.

\subsubsection{The LIF chamber}

The LIF chamber is essentially a hollow stainless-steel cylinder of external diameter $320 \mathrm{~mm}$ and length $350 \mathrm{~mm}$. Situated around the chamber perimeter are six ports for vacuum pumping, the mounting of fluorescence collection optics and for admission of the individual laser beams and the laser ablation fixture. All the principal interface ports are designed to couple to standard stainless-steel circular flanges (7.25" diameter), fitted with specific apparatus pertaining to the function of the port.

The working laser ablation flange can be attached, via a principal port, at the top of the LIF chamber. A stainless-steel tube of 0.25 " diameter sent through the flange which holds he ablation nozzle at the end of the tube. From other ends of the tube is attached to a sample reservoir. The precursors along with buffer gas coming to the nozzle 
from a gas cylinder through the sample reservoir. The bottom port of the chamber is connected to the vacuum system. The vacuum system consists of an Edward mechanical pump and a booster. The vacuum level can reach with the vacuum system is about 7 mtorr.

The left and right side of the chamber is the entry of the excitation (left side) and ablation laser (right side). Both ports are sealed with brewster plate sealing. The reason for using brewster plates is changing the polarization upon needs. The front port is used as viewing ports and as an aid in aligning the ablation laser to the slot of ablation and aligning excitation laser to the jet expansion. The backside is attached to the detection system. The fluorescence is collected by a lens that is seated $90^{\circ}$ to the excitation laser direction and then sent to the photomultiplier tube (PMT) by directing a couple of mirrors for LIF measurements. After the lens, a flip mirror is used which can be flipped down and fluorescence can be sent to the monochromator. The monochromator is used to disperse the fluorescence. The working LIF flange simply consists of a 0.25 " diameter stainless steel tube that is passed, via a compression seal, through a stainlesssteel circular flange. On the vacuum side of the flange, the tube is connected to the pulsed valve/laser ablation fixture assembly, while the other end of the tube is connected to the gas delivery system. All connections are made via Swagelok ${ }^{\circledR}$ fittings and a militarystyle electrical connection adapter is attached to the flange to supply power to the pulsed valve. 


\subsubsection{Laser ablation and supersonic nozzle}

Metal-containing free radicals were synthesized, in the gas-phase, using the laser ablation/photolysis method. In principle, the technique involves simultaneously creating two sources of highly reactive species prior to supersonic expansion. Metal atoms are generated by laser ablation of an appropriate metal target. Molecular fragments are produced via photolysis of an appropriate molecular precursor which was introduced to the ablation region by buffer gas. Through subsequent collisions, in a defined mixing region, the metals atoms and photolysis fragments can combine to form one, or more products, including the desired radicals. By varying the length of the mixing region, the number of pre-expansion collisions can be altered to enhance reactions. The extent of vibrational cooling can also be manipulated by varying the length of the mixing region.

Laser ablation was achieved using the fundamental output of an Nd:YAG laser (Continuum, Powerlite Precision II 80000). Pulse energies ranging from 10-50 mJ were routinely required to vaporize metal surfaces (the actual optimum pulse energy depending on the identity of the metal). The fundamental of YAG (1064nm) wavelength was enough to photolyze the organic or inorganic molecules, thereby potentially creating new reaction channels.

Figure 2.1 shows a schematic diagram of the stainless-steel fixture used in

the laser ablation experiments ${ }^{56}$. The ablation fixture, which is screwed on to the faceplate of a pulsed valve (General Valve Series 9), is $30 \mathrm{~mm}$ in length and incorporates a $2 \mathrm{~mm}$ diameter flow channel that passes through its central axis. In a typical experiment, a metal sample was positioned near to the exit orifice of the fixture such that its end protruded slightly into the central channel. Some metal targets could be purchased 
as $4 \mathrm{~mm}$ diameter rods and these were clamped in the fixture without any further manipulation. As a matter of routine, the metal sample was filed to obtain a clean and relatively even surface.This design of the nozzle extension was limited by signal stability. As the laser was continuously heating to the spot of the sample, it was drilling a hole through the sample. The concentration of the ablated metal atoms was varying too frequently which made the instability of the signal.

A combined gas nozzle and source material positioning/translating fixture was designed (Figure 2.2) to the stabilization of the signal ${ }^{57}$. Several design constraints were specified in order to ensure the design created the necessary conditions. Below are the design constraints 


\section{DESIGN CONSTRAINTS:}

1. Entire device must be vacuum safe.

a. Vacuum safety necessitated minimizing plastics in order to reduce outgas contamination.

b. Heat management was difficult due to the lack of conductive heat dissipation, so minimal power usage was required.

2. The device must mount on the pre-existing gas valve.

a. Overall size was tightly constrained to ensure action areas were as close as possible to the outlet flange of the valve.

b. Device weight had to be as low as possible, to avoid excess bending moment on the relatively fragile valve.

3. The device must survive incidental strikes from the ablation laser.

a. Aluminum provided sufficient heat resistance to maintain structural integrity after laser strikes, but the aluminum of the device would ablate, causing contamination of the desired samples.

b. Stainless steel, while sufficiently refractory to avoid being accidentally ablated, was heavy enough to require significant weight reduction design features.

4. It must accurately align all components, stationary, mobile and optical.

a. This constraint necessitated a strong, accurately machineable material.

b. The constant sliding motion while in use required a relatively hard material.

5. It must provide simultaneous rotary and linear motion of the rod-shaped material

a. This was required to ensure maximum usage of the material. 
b. However, the minimization of components placed in the vacuum chamber was desirable, to reduce potential contamination.

6. It must be manufacturable in the University Physics Department machine shop.

a. This machine shop is equipped with a three-axis computer-controlled milling machine, enabling complex geometries and excellent accuracy.

b. This shop is not equipped to work in exotic materials such as Inconel or ceramics.

These design constraints resulted in the ultimate device being manufactured primarily from type 303 stainless steel, due to its characteristics of excellent machinability, high refractoriness, strength, and hardness. Particular attention was paid to weight reduction by minimizing cross-sectional size and material thickness. The simple mechanism of a motor tightly coupled to a screw, operated through a fixed threaded hole, with the rod of material fixed to the end opposite the motor, provided the prescribed motion. A system of microswitches and screw-mounted flags served to signal the limits of travel, allowing the control electronics to reverse direction as required.

As many features as possible were designed into the main body, to ensure accurate alignment. The width of the main body was determined by the DC gear motor used to provide motion; with the channel, the motor slides in being just wide enough to allow free sliding motion but constraining the motor from rotary motion. The upper surface of the same channel is used to align the switching flags, constraining them to slide motion only. The threaded hole which translates the screw's rotary motion into linear motion is placed in a boss machined at the same time as the motor channel, ensuring correct placement in relation to the channel. Simple set-screw couplers were 
used to connect the motor to the leadscrew, and the leadscrew to the source material rod. The leadscrew was manufactured from a standard type 316 stainless steel threaded rod. Once the design was finalized with simulation and test, it was manufactured on a HAAS Toolroom CNC vertical machining center. The main body was milled from a single piece of material in several setups, each carefully aligned to ensure the accuracy of the final part. The several supporting pieces were individually machined on either the HAAS VMC or using appropriate manual machine tools. Final finishing and assembly were accomplished by hand, with each part being fitted to the rest to guarantee smooth, accurate function.

To drive the gear motor a homemade circuit was designed and below is the description of the circuit:

The circuit shown in figure 2.3 generates Pulse Width Modulated (PWM) signal using a 555 timer. The timer is configured to operate in astable mode. An RC circuit (R1 and C2) is connected to the output of the timer that controls the state of the timer by sequentially charging and discharging the capacitor, C2. The charging and discharging path is defined using two diodes D1 and D2. During the charging cycle, the capacitor C2 gets charged through the right side of the variable resistor R1 $(100 \mathrm{k} \Omega$ potentiometer). And during the discharging cycle, capacitor C2 gets discharged through left side of the variable resistor. By changing the ratio of left-side and right-side resistance of the potentiometer, the charging and discharging time can be varied while keeping the frequency of the signal constant. So, we get a pulse width modulated (PWM) signal from the timer operation where the pulse width is controlled by the $100 \mathrm{k} \Omega$ potentiometer, R1. The generated PWM signal is gated through two AND gates and 
subsequently fed to a Motor Driver Module (MDM) that drives a gear motor. A flip flop controls the AND gates to let the PWM signal pass through one gate at any instance. The flip flop is set or reset by two external switches. The switches are normally connected to ground that does not change the state of the flip flop (as shown in table 2.1 as $5^{\text {th }}$ state). But when one of the switches is actuated (i.e. it becomes high), the flip flop state changes (as shown in the table as of $6^{\text {th }}$ and $7^{\text {th }}$ state). Each AND gates have one input connected to Q or Q' of the flip flop. Since at any instance either Q or Q' is high, only one of the AND gates can pass the PWM signal through the gates. The AND gate output goes to Motor Driving Module. IN1 and IN2 pins of the MDM are connected to the AND gate outputs. The Motor Driving Module operates in different operating modes based on the input pin signals as shown in table2.2. When IN1 is fed the PWM signal, it drives the motor forward and when IN2 is fed the PWM signal, it drives the motor backward. In addition, the pulse width determines the power given to the motor that controls the speed of the motor rotation. In summary, the switches control the direction of the motor rotation whereas the potentiometer (by changing the pulse width) controls the speed of the motor rotation.

The motorized stage nozzle extension made the experimental set much better in terms of signal stability and removes all difficulties of data taking procedure. The electrical circuit finally modified using an Arduino-UN microcontroller along with an L298 dual H-Bridge motor control module. Nowadays Arduino is very famous as the coding is very simple and straight forward. In our experimental apparatus as the direction control was done using microswitches and inside the vacuum chamber with a vacuum environment, the switches were dying easily that's why we replaced the circuit with an 
Arduino based circuit. Figure-2.4 representing the circuit component and code can be found in appendix A. In this new version of the motorized stage circuit, no feedback from the motor is needed to change the direction of the motor. Instead, the time duration was adjusted for the full use of the calcium rod. No micro-switches are needed for changing the direction. 


\subsubsection{Supersonic jets and molecular beams}

Spectroscopic applications of supersonic jets were less common until it was recognized that jet cooling could be accomplished to simplify molecular spectra. Band origins, vibrational progressions, and rotational structure can be much more readily identified due to the depopulation of rotational, and often vibrational, energy levels ${ }^{58}$.

However, until the early 1970's, most of the molecular spectroscopy had dealt with stable molecules that could be identified using slow detection techniques in higher vibrational or rotational temperature. It was later perceived that supersonic jets provide an excellent environment for the preservation of short-lived species, although spectroscopic observation was difficult until the commercial availability of laser. The supersonic jet in molecular spectroscopy to study free radical rapidly expanded in recent years and, at present, is one of the most important areas of spectroscopic research ${ }^{59}$.

In our laboratory, supersonic free-jet expansion, which provides cooling of the rotational temperature ( $\mathrm{T} \sim 30 \mathrm{~K}$ ), is formed using a commercial pulsed valve (General Valve 9 Series) with typically a $0.50 \mathrm{~mm}$ (sometimes $.30 \mathrm{~mm}$ ) circular orifice. Typically, helium or argon with 200 psi backing pressure passes through the sample bomb. The inert gases behave as buffer gas which introduces organic precursor to the nozzle i.e. in the jet.The supersonic jet in molecular spectroscopy is extremely important in cooling molecules. Spectra involving complex vibrational and rotational structure associated with room temperature, or warmer molecules, can be greatly simplified under supersonic conditions. 
In practice, a perfect free supersonic jet expansion can never be obtained as to achieve this requires expansion of the gas into a perfect vacuum. The extent of the favorable properties of a supersonic jet is in nominally depends on the pumping speed of the vacuum pump. Any residual background gas (especially leaks) will severely disrupt the properties of the jet by creating turbulence effects. Pulsed nozzle supersonic jets are used in spectroscopic work as pulsed lasers are employed, and in this case the demands on the pumping system are much less strenuous. This type of supersonic expansion was routinely used throughout the work described in this thesis. 


\section{$2.2 \quad$ Electronic spectroscopy}

In this section, the techniques of laser-induced fluorescence (LIF) excitation spectroscopy and dispersed fluorescence (DF) spectroscopy will be discussed. The sensitivity of these techniques is ideal for studying gas-phase free radicals.

\subsubsection{Laser-induced fluorescence (LIF) spectroscopy}

Laser-induced fluorescence (LIF) excitation ${ }^{8}$ is widely used and a relatively simple spectroscopic technique for the investigation of optically excited states in both atoms and molecules. Its high sensitivity is one of the major reasons for the rapid advancement of this technique over the last thirty years. Combining supersonic jet technology with the LIF technique has also had a profound effect on the quality of molecular spectra. Low resolution, supersonically cooled LIF spectra, recorded using laser linewidths of above $\sim 1 \mathrm{~cm}^{-1}$, routinely yield much clear, uncongested vibrational spectra. Higher-resolution spectra can provide fully rotationally resolved spectra for many small and medium-sized molecules.

In principle, any molecule absorbs a photon of light, resulting in promotion to a higher energy electronic state. Conventional absorption techniques detect molecular absorption by monitoring the decrease in light intensity on passage through a sample of molecules. A limitation is introduced on this technique by the ability of the detector to observe small changes in the intensity of light. Clearly, if a laser was used as the light source in such an experiment, small changes in the high laser intensity would be difficult to detect. 
Laser-induced fluorescence relies on the ability of an excited electronic state to decay via spontaneous emission of a photon. Fluorescence is distinctly proof of the initial absorption process and its detection is, therefore, an indirect observation of molecular absorption. One negative aspect of LIF spectroscopy is that not all excited states decay radiatively. Fluorescence spectroscopy will experience if other decay processes have a rate comparable to, or greater than spontaneous emission. Figure 2.5 illustrates the different decay processes that can potentially occur following the absorption of a photon. In this work, the laser beam from a tunable dye laser (SpectraPhysics, Cobra Stretch) was directed into the emerging supersonic expansion, 15-20 mm downstream from the end of the laser ablation fixture.

In a typical experiment, the laser wavelength was scanned until it resembled the energy difference between two molecular energy levels. At these wavelengths absorption of a laser, photon could occur, promoting the molecule to an excited electronic state. Fluorescence at many wavelengths can occur following excitation corresponding to decay to various rovibrational levels in lower-lying electronic states, including the ground state. Figure 2.6 illustrates the absorption and fluorescence processes involved in LIF spectroscopy. Since the fluorescence is observed as a means of monitoring the absorption process, the total fluorescence can be detected. Fluorescence was collected, perpendicularly to the laser beam, via an f/1.5 lens and imaged onto a photomultiplier tube (PMT, Hamamatsu, H10721-01) ${ }^{60,61}$. Positioning the PMT at right angles to the beam helped to minimize scattered light noise and, therefore, gave an improved sensitivity. The output from the PMT was integrated by a BoxCarr and then sent to PC by analog to digital converter. The digitized signal was passed to the PC on a shot-to-shot 
basis. An excitation spectrum was obtained by plotting the total fluorescence intensity as a function of laser wavelength. The resulting spectrum is similar to a conventional absorption spectrum, although the signal intensity depends not only on the absorption probability but also on the excited state fluorescence quantum yield. The spectral resolution was governed by the linewidth of the exciting dye laser and typically spectra were recorded with a resolution of $0.1 \mathrm{~cm}^{-1}$. 


\subsubsection{Dispersed fluorescence (DF) spectroscopy}

A complimentary technique that is often used in concurrent with LIF excitation spectroscopy is dispersed fluorescence (DF) spectroscopy. Dispersed fluorescence usually yields information of ground electronic states, but also can be used to investigate low-lying excited electronic states. Dispersed fluorescence can be an important aid in the assignment of vibrational structure in the excited state for complicated excitation spectra, in which the vibrational assignment remains vague. This technique can also be used to elucidate the vibrational manifold of the ground and lowlying excited electronic states. In a typical dispersed fluorescence experiment, the laser wavelength can be tuned to a specific electronic-vibrational transition, the so-called pump position, resulting in selective excitation to the excited state (figure 2.7). The resulting fluorescence was directed through the entrance slits of an Acton Research, SpectraPro 300i dispersing monochromator via a series of optics ${ }^{3}$. The monochromator is equipped with an intensified CCD (iCCD) camera (Princeton Instruments, PI-MAX 512). A typical spectral resolution of $\sim 20 \mathrm{~cm}^{-1}$ can be observed. The frequency of the monochromator was calibrated using a mercury arc lamp. The frequency uncertainty of the DF spectra is limited by the resolution of the monochromator to $\sim 5 \mathrm{~cm}^{-1}$. A schematic diagram of the LIF/DF experiment is illustrated in figure 2.8. 


\subsubsection{Experimental synchronization}

Since almost all the parts of the apparatus were pulsed $(10 \mathrm{~Hz})$, one of the most important features of an experiment was the accurate synchronization of all the pulses. The length and timing delays between various pulses were crucial for the successful implementation of an experiment, and therefore, pulse-generation electronics were used to control the experimental apparatus. Table 2.3 and figure 2.8 shows the delay timing and schematic diagram of the timing aspects of a typical experiment and outline some of the particular pulse lengths and timing delays. Typically, the opening of the pulsed valve (nozzle input) defined the start of an experiment $(\mathrm{t}=0)$, or trigger pulse. The firing of the pulsed valve was controlled by a General Valve Iota One pulse driver. Control of the firing times of the Nd:YAG and tunable dye laser were achieved using a Stanford Research Systems DG365 Digital Delay/Pulse Generator. The Nd:YAG laser, which pumps the dye laser, was set to fire approximately 25 us after the ablation laser(continuum YAG), in order to probe the appropriate part of the supersonic expansion. Since shot averaging was employed in the data acquisition process, an appropriate stop-pulse was required by the dye laser, instructing it to move the cavity diffraction grating to a new position only when sufficient sampling had occurred at the current wavelength. An appropriate stop-pulse was also sent to the digitizer, instructing it to cease data sampling while the diffraction grating was moving to its new position. Since the optimum pulse lengths and delays varied from experiment to experiment, all of the timing delays were manually adjusted to visually optimize the real-time laser-induced fluorescence signal on a Tektronix DPO 3014 digital oscilloscope. 


\subsection{Cavity ring-down spectroscopy}

Many molecules and free radicals including metal-containing ones don’t have sufficient fluorescence quantum yields to be studied by LIF spectroscopy. In the future, we will use CRDS combined with laser ablation nozzles to study such free radicals.

\subsubsection{Principle of cavity ring-down spectroscopy (CRDS)}

$$
\text { Cavity Ring-down spectroscopy (CRDS) is a multipass absorption }
$$
spectroscopic technique useful for observing relatively weak spectroscopic transitions. The most significant advantage of the CRDS technique is its high sensitivity. In a traditional absorption experiment, absorption of light by a sample is determined by BeerLambert law.

$$
\mathrm{I}=\mathrm{I}_{0} \cdot \exp (-\sigma \mathrm{NL})
$$

where $\mathrm{I}_{0}$ and $\mathrm{I}$ are the intensity of incident and transmitted light, $\sigma$ is the absorption cross section of the molecule at a given wavelength, $\mathrm{N}$ is the number density of the molecules, and $\mathrm{L}$ is the path length of the region where light interacts with the absorbing species. Instrumental sensitivity of an absorption experiment is often limited by the path length and/or the sample concentration, which makes detecting weak transitions infeasible. CRDS provides a significant increase in instrumental sensitivity by increasing the path length, which makes it ideal to study weak transitions. The principle behind CRDS is a

cavity defined by two highly reflective mirrors that are carefully aligned such that light injected into this cavity through one of the mirrors will be reflected back and forth 
between the mirrors greatly increasing the effective path length of the light through the sample (Figure 2.9). A very small portion of the trapped photons leaks through each of the mirrors on each round trip. The light leaking out of the cavity decreases exponentially with consecutive round trips and the intensity of the radiation leaked out is measured as a function of time and recorded. The decay curve of the intensity as a function of time is fit to an exponential function, yielding the ring-down time.

Beer-Lambert law can be adapted to calculate the fractional loss of the intensity of light in a cavity.

$$
\left.I_{t}=I_{0} \cdot \exp \left[-\left(\frac{\text { loss }}{\text { reflection }}\right)\left(\frac{\text { number of reflections }}{\text { round trip }}\right) \text { (number of round trips }\right)\right]
$$

The number of reflections per round trip is 2 , because of two mirrors in a cavity. The number of round trips (n) in time $t$ would then be given by

$$
\mathrm{n}=\frac{\mathrm{tc}}{2 \mathrm{~L}}
$$

where $\mathrm{L}$ is the length of the ring down cavity and $\mathrm{c}$ is the speed of light. If $\mathrm{R}$ is taken as the reflectivity of the mirrors, then the loss per reflection would be (1-R). Eq. (3.2) can then be modified as

$$
\mathrm{I}_{\mathrm{t}}=\mathrm{I}_{0} \cdot \exp \left[-(1-\mathrm{R})\left(\frac{\mathrm{tc}}{\mathrm{L}}\right)\right]
$$

The intensity of light $\left(\mathrm{I}_{\mathrm{t}}\right)$ then decays with a time constant, viz, the empty-cavity ringdown time, $\tau_{0}$, which is defined as the time for the intensity to decay to the $1 / \mathrm{e}$ of the initial intensity $\left(\mathrm{I}_{0}\right)$. In an empty cavity, $\tau_{0}$ is given by 


$$
\tau_{0}=\frac{\mathrm{L} / \mathrm{C}}{(1-\mathrm{R})}
$$

When an absorbing sample is introduced inside the cavity, the loss of intensity of light due to absorption is given by

absorption loss $=($ loss per round trip $)($ number of round trip $)=(2 \sigma \mathrm{Nl}) \frac{\mathrm{tc}}{2 \mathrm{~L}}$

where $l$ is the length of the absorbing sample inside the cavity which could be smaller or equal to the length of the cavity L. The total loss in the intensity of light is then given by

$$
\text { total loss }=(1-\mathrm{R})\left(\frac{\mathrm{tc}}{\mathrm{L}}\right)+(\sigma \mathrm{Nl})\left(\frac{\mathrm{tc}}{\mathrm{L}}\right)=[(1-\mathrm{R})+(\sigma \mathrm{N} 1)] \frac{\mathrm{tc}}{\mathrm{L}}
$$

Beer-Lambert law for a rind-down cavity with an absorber is therefore:

$$
\mathrm{I}_{\mathrm{t}}=\mathrm{I}_{0} \cdot \exp \left\{-[(1-\mathrm{R})+\sigma \mathrm{Nl}]\left(\frac{\mathrm{tc}}{\mathrm{L}}\right)\right\}
$$

The ring-down time of the cavity with the absorbing sample, $\tau_{\mathrm{abs}}$, is given by

$$
\tau_{\mathrm{abs}}=\frac{\mathrm{L} / \mathrm{C}}{(1-\mathrm{R})+\sigma \mathrm{Nl}}
$$

Eqn. (3.5) and (3.9) can then be combined to give

$$
\sigma \mathrm{Nl}=\frac{\mathrm{L}}{\mathrm{C}}\left(\frac{1}{\tau_{\mathrm{abs}}}-\frac{1}{\tau_{0}}\right)
$$

There are two main benefits of CRDS when compared with typical absorption spectroscopy experiments. The first is the greatly increased effective path length and hence the extremely high sensitivity. For a typical flow-cell CRDS experimental setup, 
$\mathrm{L}=\mathrm{l}=0.5 \mathrm{~m}, \mathrm{R}=99.995 \%$, the empty-cavity ring-down time is $33 \mu \mathrm{s}$ and the effective path length is $10 \mathrm{~km}$. Thanks to the long path length, a minimum detectable absorption coefficient of $\sim 0.3$ ppm per pass can be achieved.

The second major advantage of CRDS is the insensitivity to fluctuations in incident radiation intensity. Unlike in traditional multipass absorption spectroscopy, in which intensity of the transmission is used to determine the absorbance, in CRDS, it is determined from change of the decay constant when the absorbing sample is introduced into the cavity. The intensity of the incident beam, therefore, doesn't directly affect the absorption cross section measurement. 


\subsubsection{Experimental setup}

The experimental setup consists of a pulsed laser source, motorized ablation nozzle extension with general valve nozzle and an optical cavity. Second harmonic output $(532 \mathrm{~nm})$ from an Nd:YAG laser (Spectra-Physics Quanta-Ray 270, repetition rate $=20 \mathrm{~Hz}$ ) is used to pump a tunable dye laser (Sirah, Precision Scan). If needed, the visible output from the dye laser can be extended into the near-IR (NIR) and mid-IR (MIR) regions by stimulated Raman scattering (SRS) or difference-frequency generation (DFG), respectively. The laser beam is collimated by a telescope consisting of two confocal lenses ( $\mathrm{f}=75$ and $50 \mathrm{~mm}$ ) and before being injected into the ring-down cavity. A $100 \mu \mathrm{m}$ pinhole is placed at the focal point of the telescope for mode selection. The ringdown cell is $67 \mathrm{~cm}$ in length and it is formed by a pair of commercial cavity ring-down mounts and mirrors (Los Gatos Research) attached to the ends of two arms of a vacuum chamber evacuated by a mechanical booster pump (Edwards, $\left.1200 \mathrm{~m}^{3} \mathrm{~h}^{-1}\right)^{62-64}$. The mirrors are kept clean by continually flushing with $\mathrm{N}_{2}$. The transmitted radiation leaking from the exiting CRD mirror is collected by a focusing lens and detected by a PMT (Hamamatsu, H10721-01, for UV-visible) or an InGaAs Photodiode (Thorlabs, PDA400, for IR). Long-pass filters are used in front of the detectors to block background radiation. The ring-down signal is acquired with an oscilloscope (Tektronix, DPO3014) and sent to a PC for processing and recording. Figure 2.10 shows a schematic for jet-cooled experimental apparatus. For the ablation laser source, another non-q-switched YAG laser will be used ${ }^{65}$.

A LabVIEW program has been developed for data acquisition, fitting the ring-down curve to an exponential function, calculating absorbance, as well as 
controlling wavelength scan of the Sirah laser. Ring-down curves are averaged on the oscilloscope (typically by 32 times) before sent to the PC via a USB port. Further averaging of ring-down curves (typically by 32 times) may be performed by the LabVIEW program. Extraction of ring-down times is based on a Levenberg-Marquardt algorithm (programmed and complied in C and embedded in the LabVIEW program) allowing the shot to shot (at a repetition rate of $20 \mathrm{~Hz}$ ) analysis of up to 10,000 data points. The program also allows fitting the transient decay in several (typically three) sections, offering the possibility of extracting multiple ring-down times thereby dealing with nonexponential behavior. At each wavelength, multiple averaged ring-down curves are fit, and the ring-down times derived, based on which the standard deviation (rootmean-square) is calculated ${ }^{64}$.

In order to remove background absorption and absorption due to the precursor, CRD signals with the ablation laser on and off were obtained at each laser wavelength, which is done by TTL control pulses generated by the LabVIEW program and sent via a serial port of the PC. The CRD spectrum with ablation off is then subtracted from that with ablation on to obtain the net spectrum due to free radicals. Delay and pulse generators (Stanford Research Systems, DG645, and DG535) are used to synchronize the Nd:YAG laser pulses, the dye laser scan, jet expansion, and data acquisition. Figure 2.9 and Table 2.4 is illustrating the time aspects and the time scale used in the CRDS experiment. 


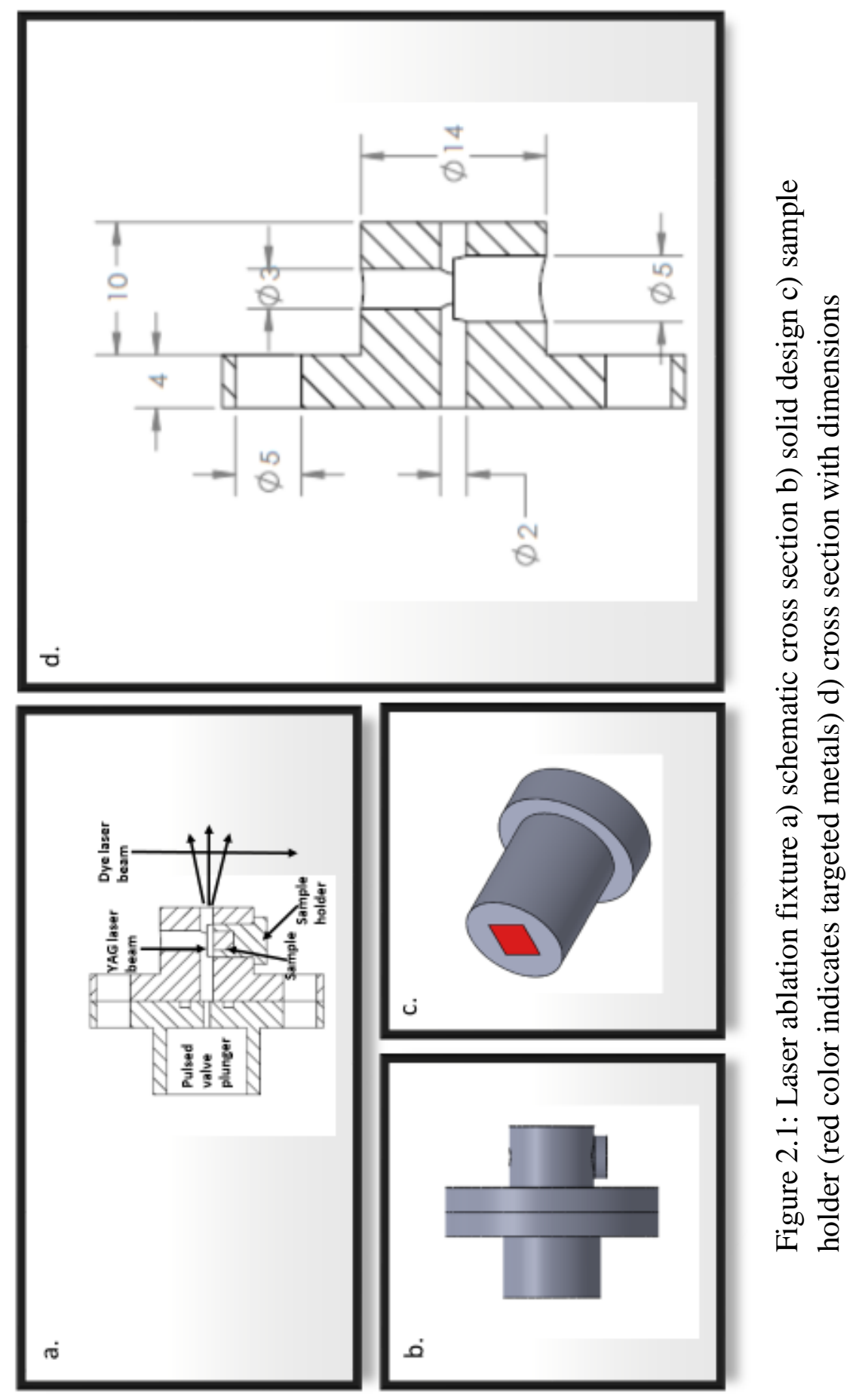




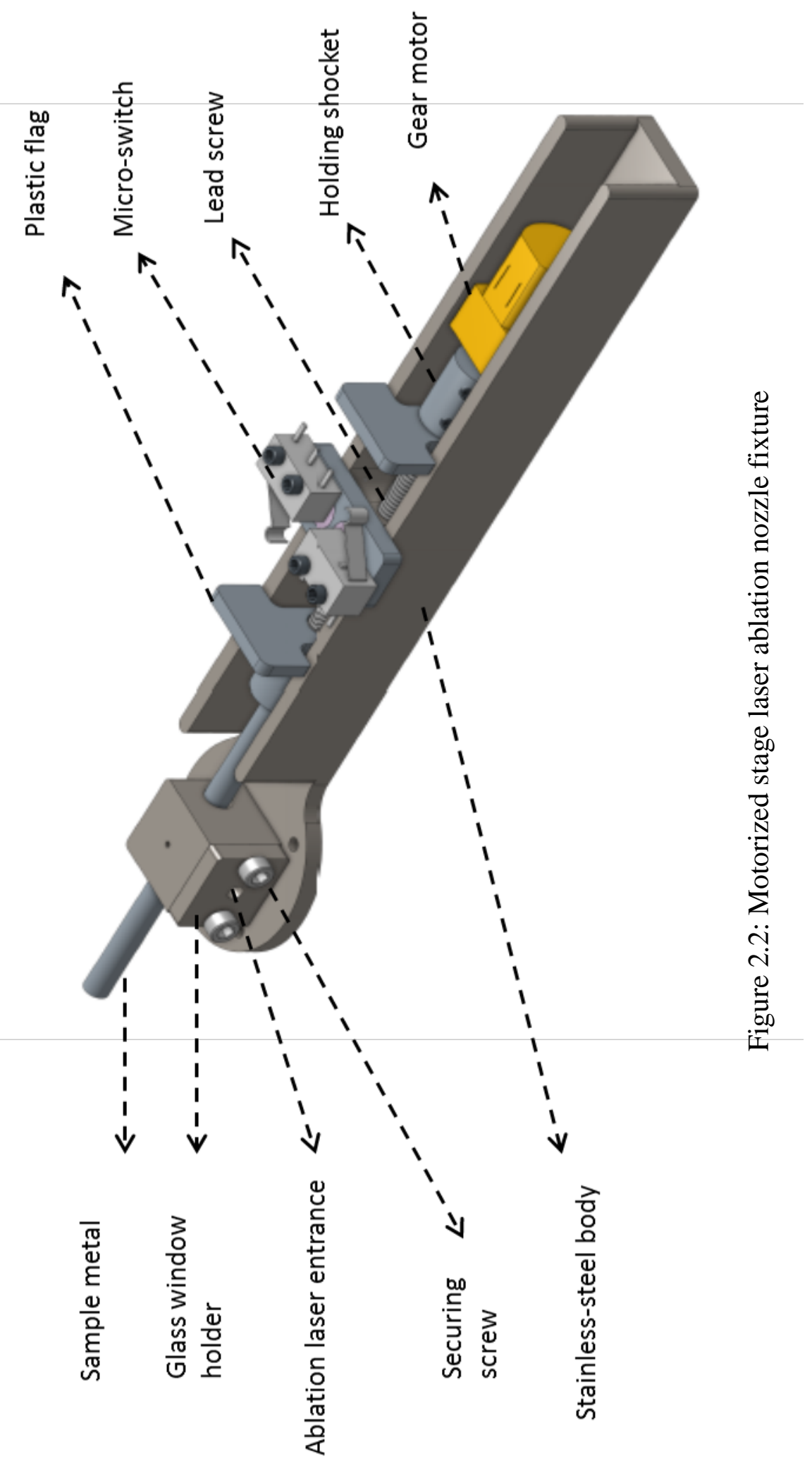




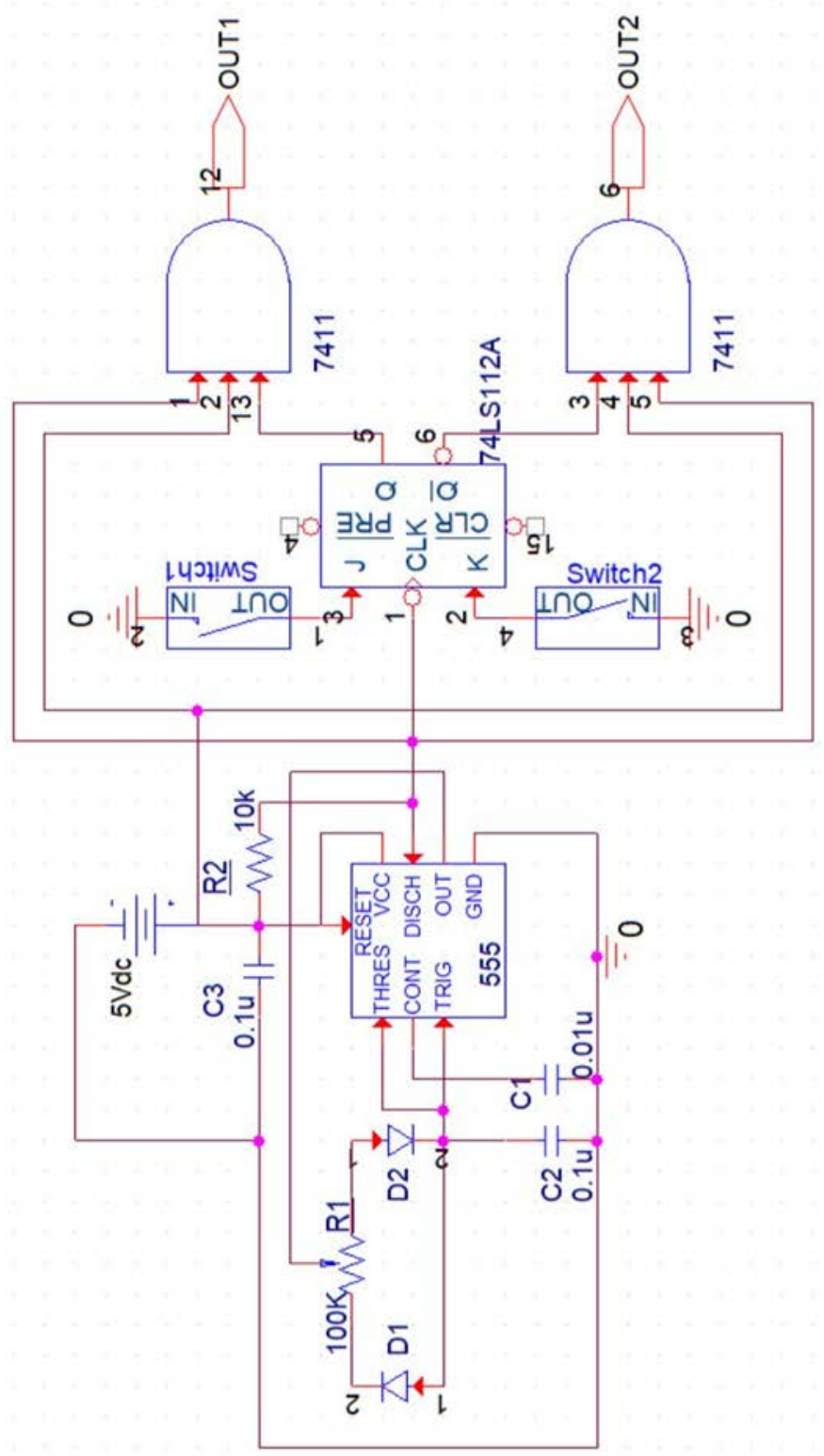

Figure 2.3: Block diagram of electrical circuit for gear motor control 
Table 2.1: Truth table of JK flipflop

\begin{tabular}{|c|c|c|c|c|c|c|c|}
\hline \multirow[t]{2}{*}{ Configurations } & \multicolumn{5}{|c|}{ Inputs } & \multicolumn{2}{|c|}{ Outputs } \\
\hline & $\underline{\text { PRE }}$ & CLR & CLK & $\mathbf{J}$ & $\mathbf{K}$ & $\mathbf{Q}_{0}$ & $\underline{\mathbf{Q}_{0}}$ \\
\hline 2 & $\mathbf{L}$ & H & $\bar{x}$ & $x$ & $x$ & H & $\mathbf{L}$ \\
\hline 3 & $\mathbf{H}$ & $\mathbf{L}$ & $x$ & $x$ & $x$ & $\mathbf{L}$ & $\mathbf{H}$ \\
\hline 4 & $\mathbf{L}$ & $\mathbf{L}$ & $\bar{x}$ & $x$ & $x$ & H & H \\
\hline 5 & $\mathbf{H}$ & $\mathbf{H}$ & $\downarrow$ & $\mathbf{L}$ & $\mathbf{L}$ & $\overline{Q_{0}}$ & $\underline{\mathbf{Q}_{0}}$ \\
\hline 6 & $\mathbf{H}$ & $\mathbf{H}$ & $\downarrow$ & $\mathbf{H}$ & $\mathbf{L}$ & $\mathbf{H}$ & $\mathbf{L}$ \\
\hline 7 & $\mathbf{H}$ & $\mathbf{H}$ & $\downarrow$ & $\mathbf{L}$ & $\mathbf{H}$ & $\mathbf{L}$ & H \\
\hline 8 & $\mathbf{H}$ & H & $\downarrow$ & H & $\mathbf{H}$ & Toggle & Toggle \\
\hline 9 & $\mathbf{H}$ & $\mathbf{H}$ & $\mathbf{H}$ & $x$ & $x$ & $\overline{Q_{0}}$ & $\underline{\mathbf{Q}_{0}}$ \\
\hline
\end{tabular}

Table 2.2: Motor driver module motor driving modes

\begin{tabular}{|c|l|c|c|}
\hline DC MOTOR & MODE & IN1 & IN2 \\
\hline MOTOR-A & Forward & $1 /$ PWM & 0 \\
\hline & Reversion & 0 & $1 /$ PWM \\
\hline & Standby & 0 & 0 \\
\hline & Brake & 1 & 1 \\
\hline \hline
\end{tabular}




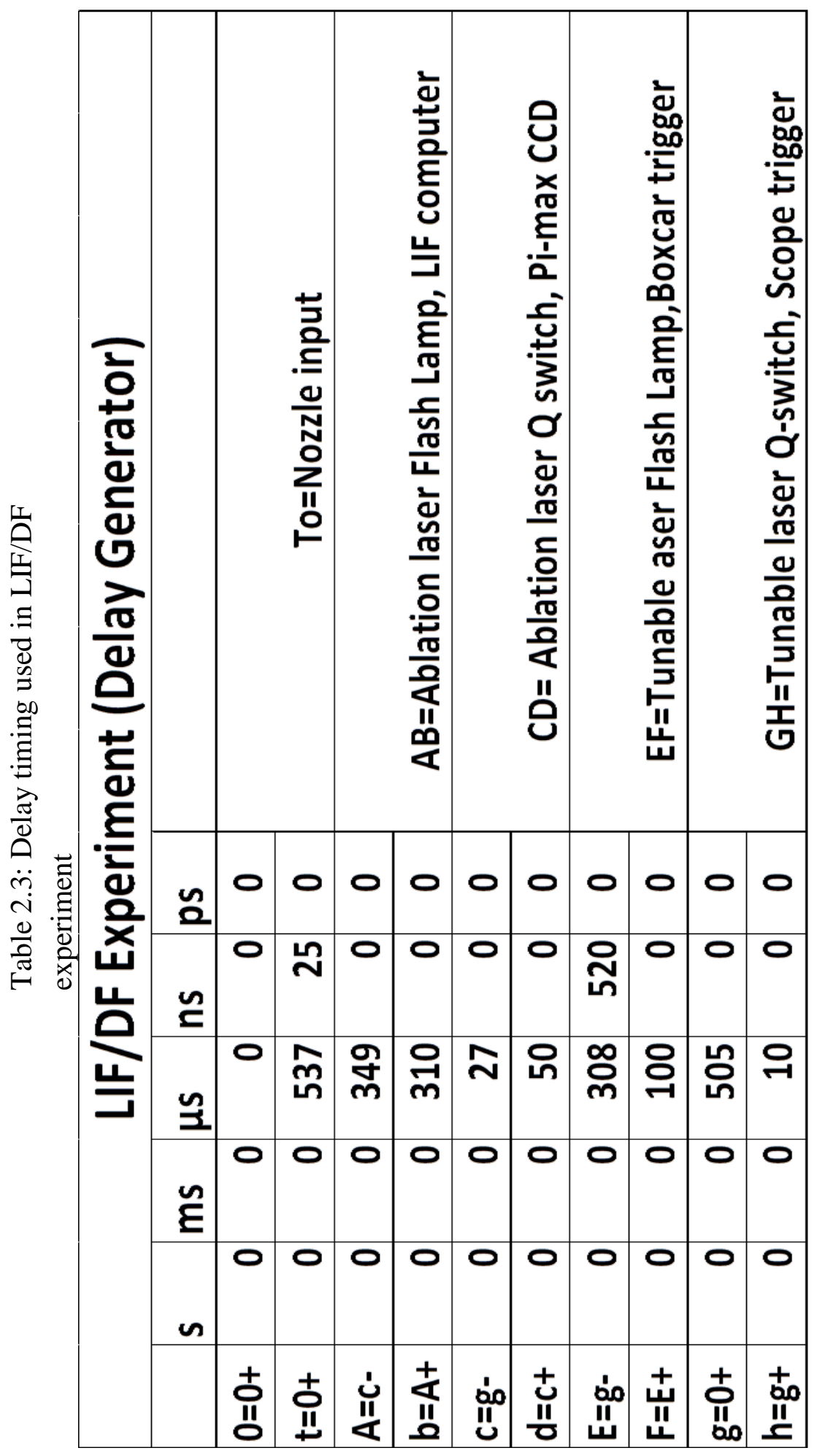




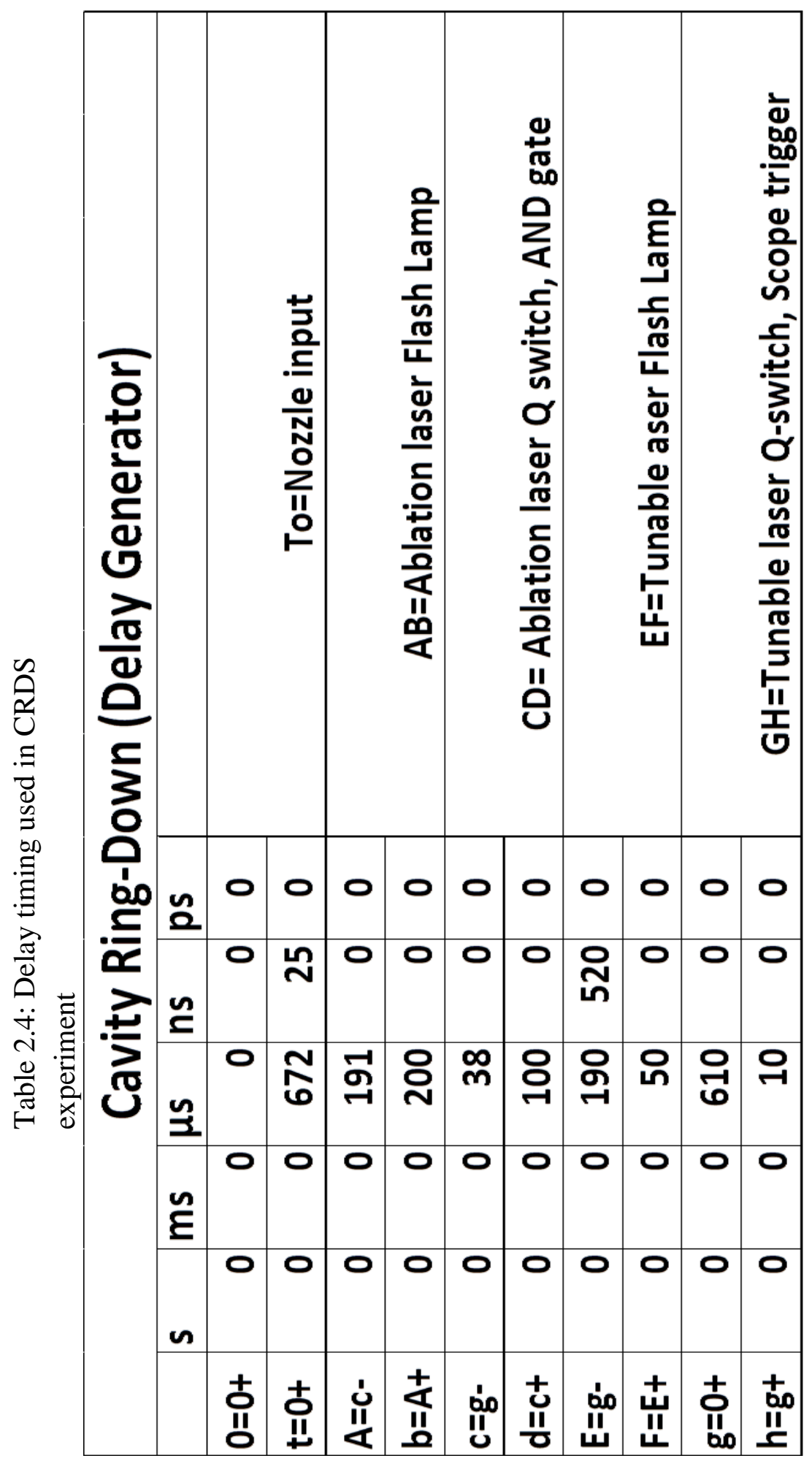




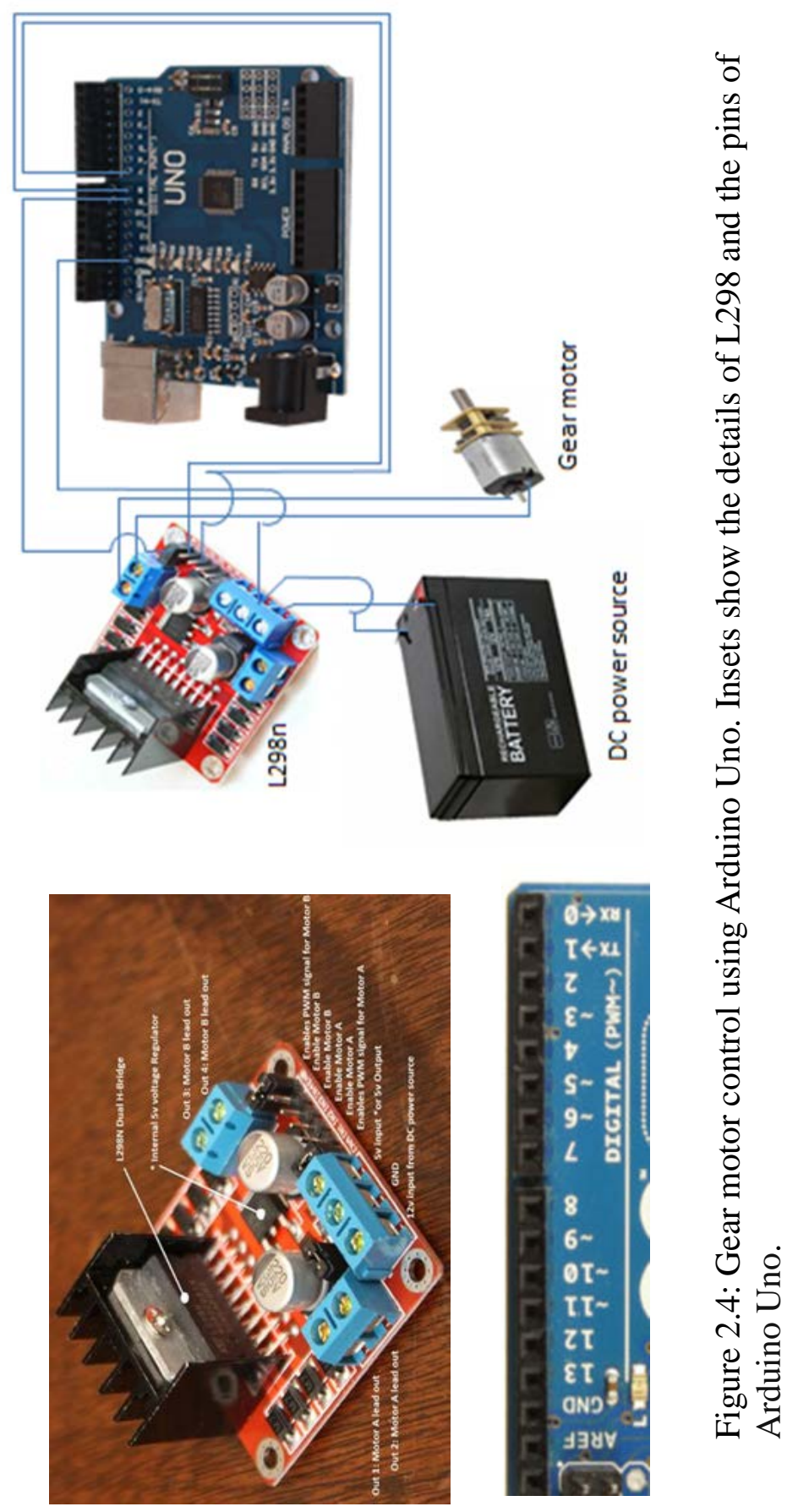




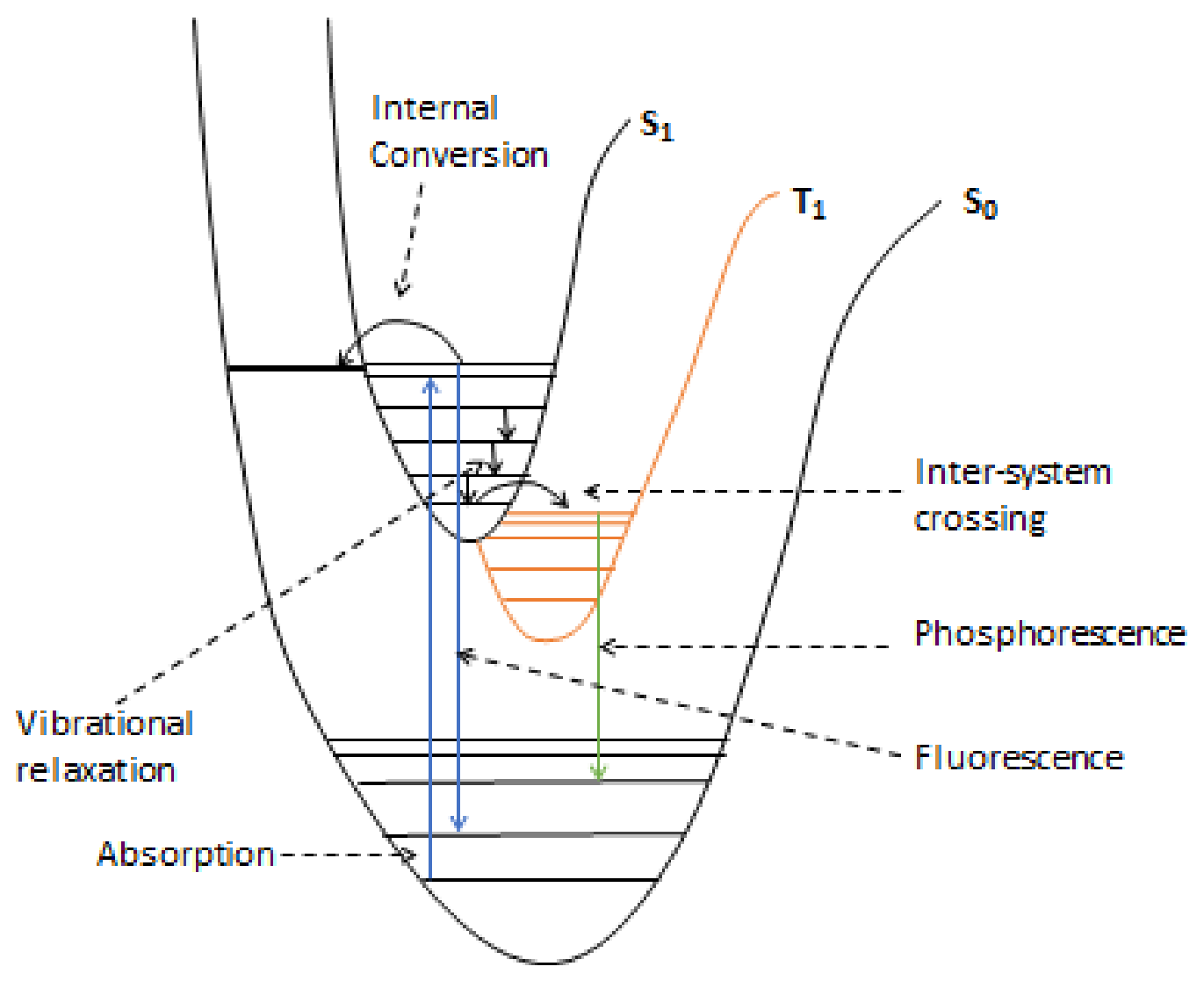

Figure 2.5: Decay process which can occur following an absorption process 


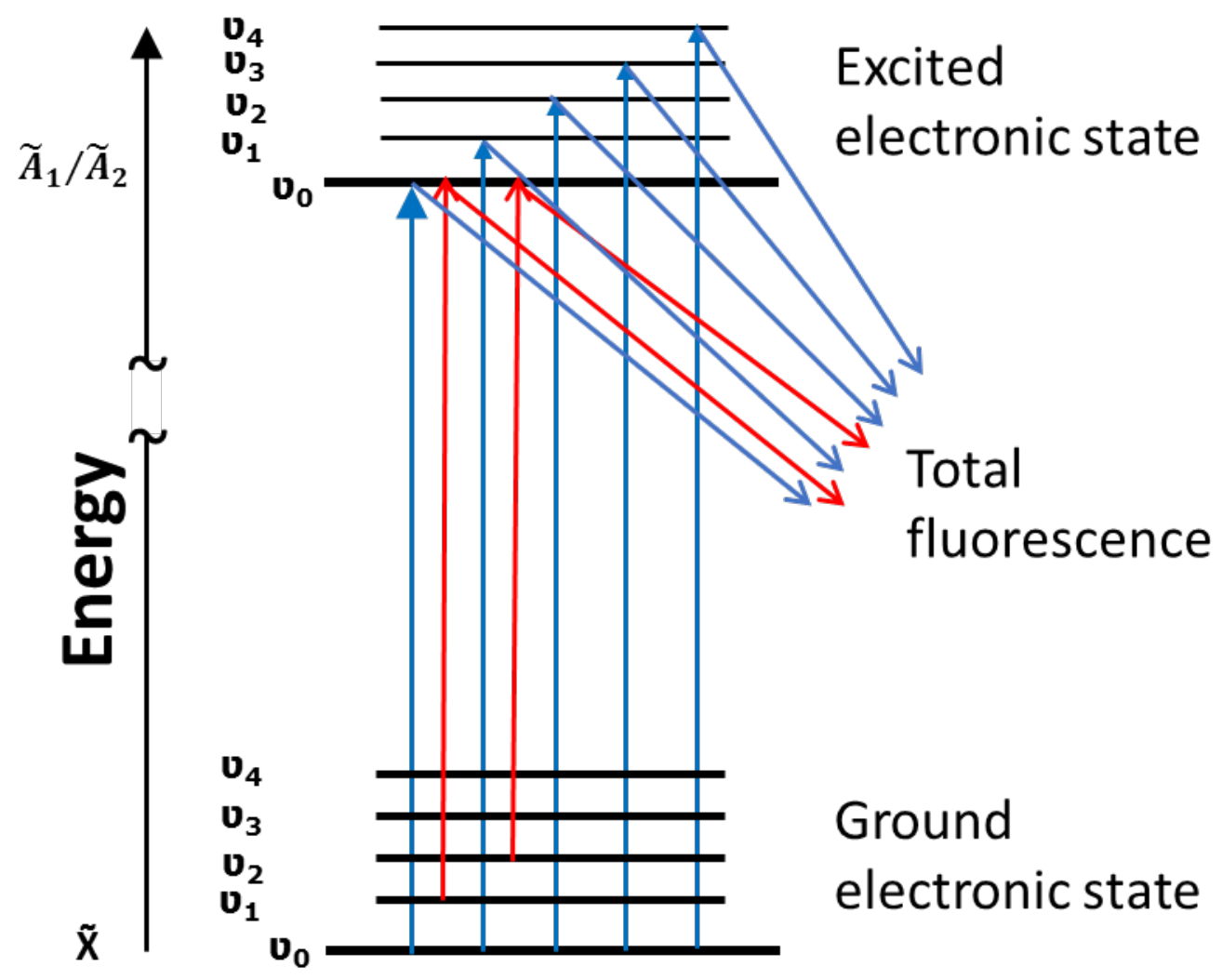

Figure 2.6: Schematic diagram showing the fundamental transitions involved LIF excitation spectroscopy. Red arrows indicate hot band transitions 


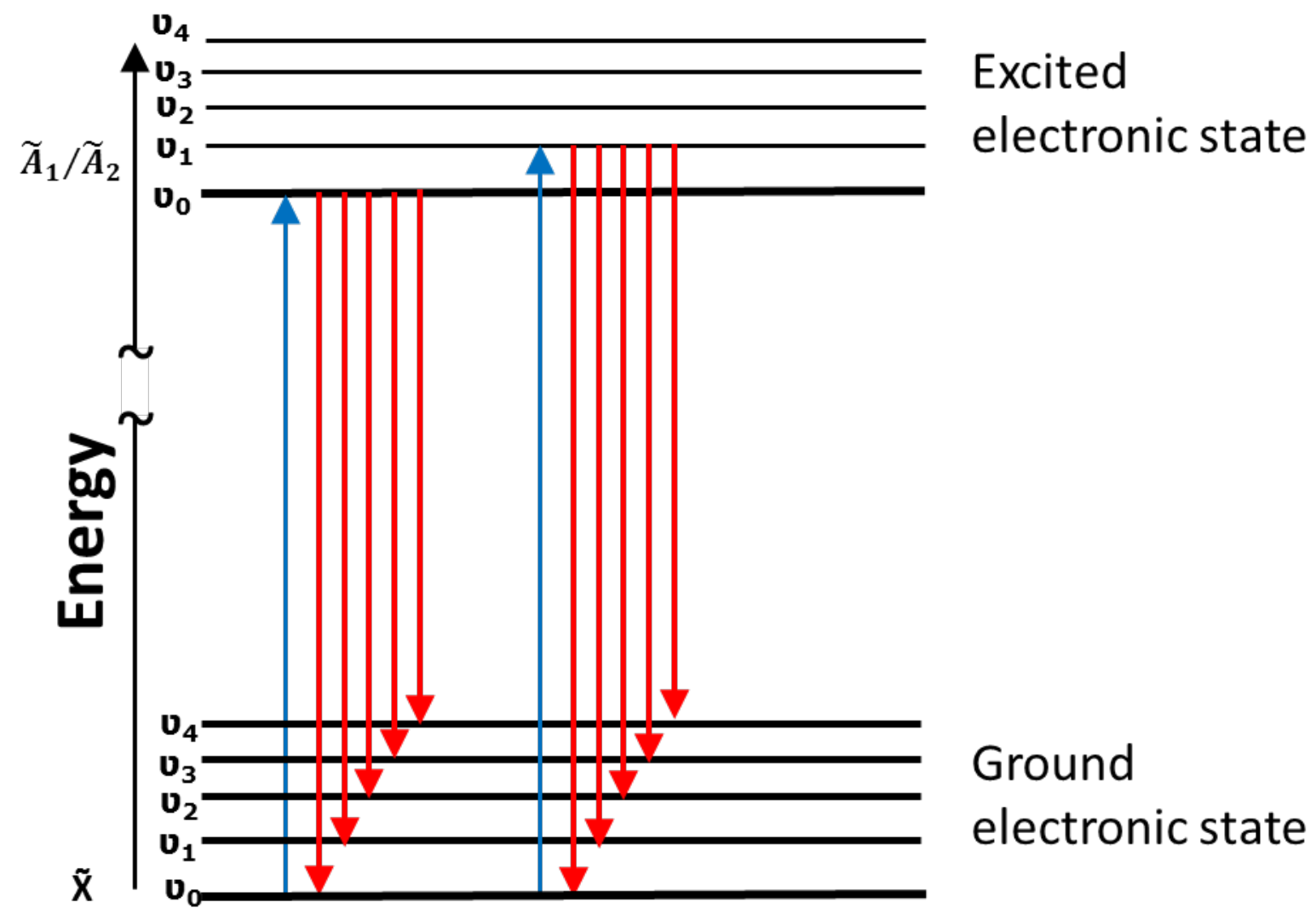

Figure 2.7: Transitions involved in laser-induced dispersed fluorescence (DF) spectroscopy. The red transition represents the laser excitation, or pump, transition 


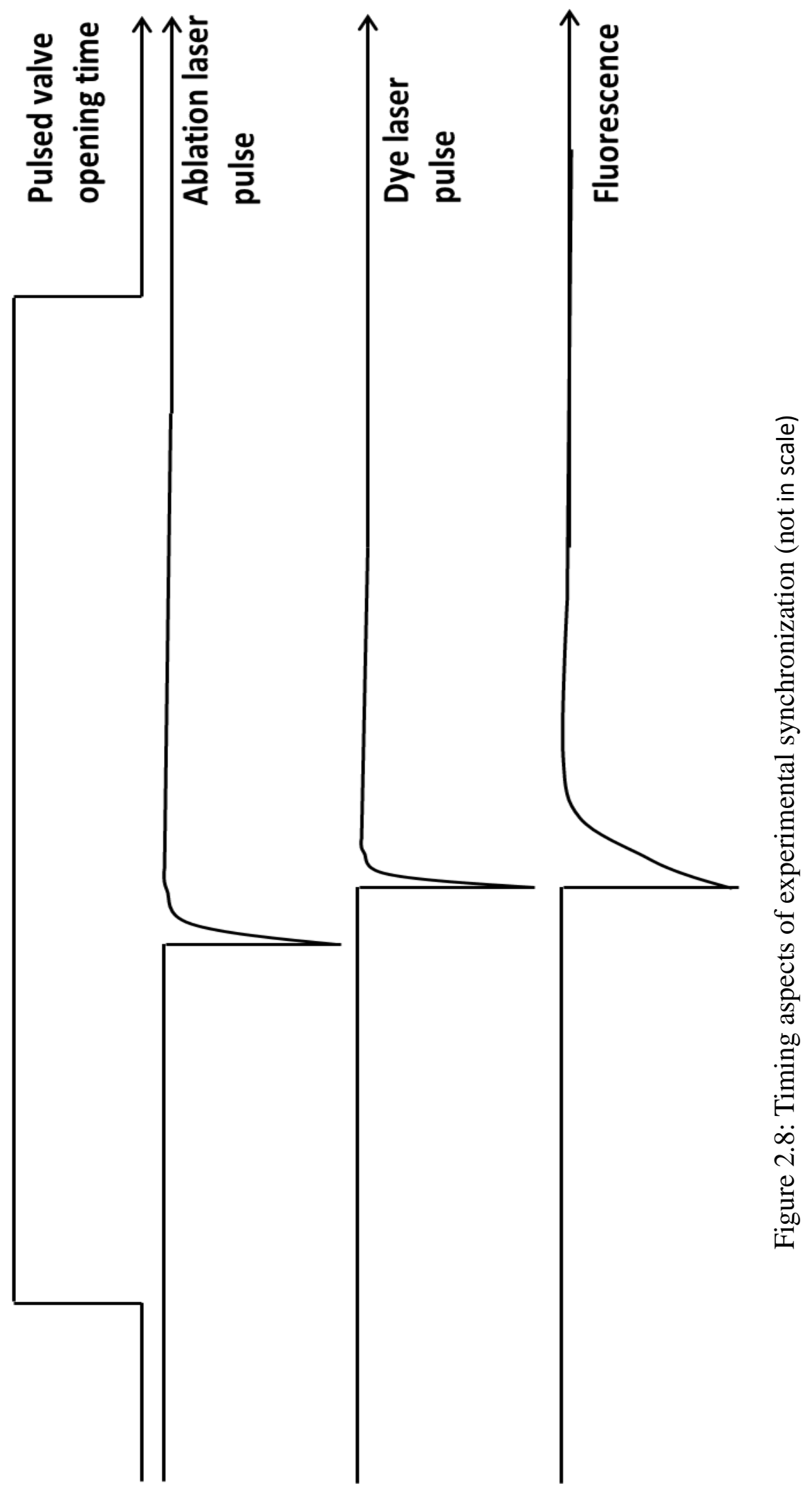




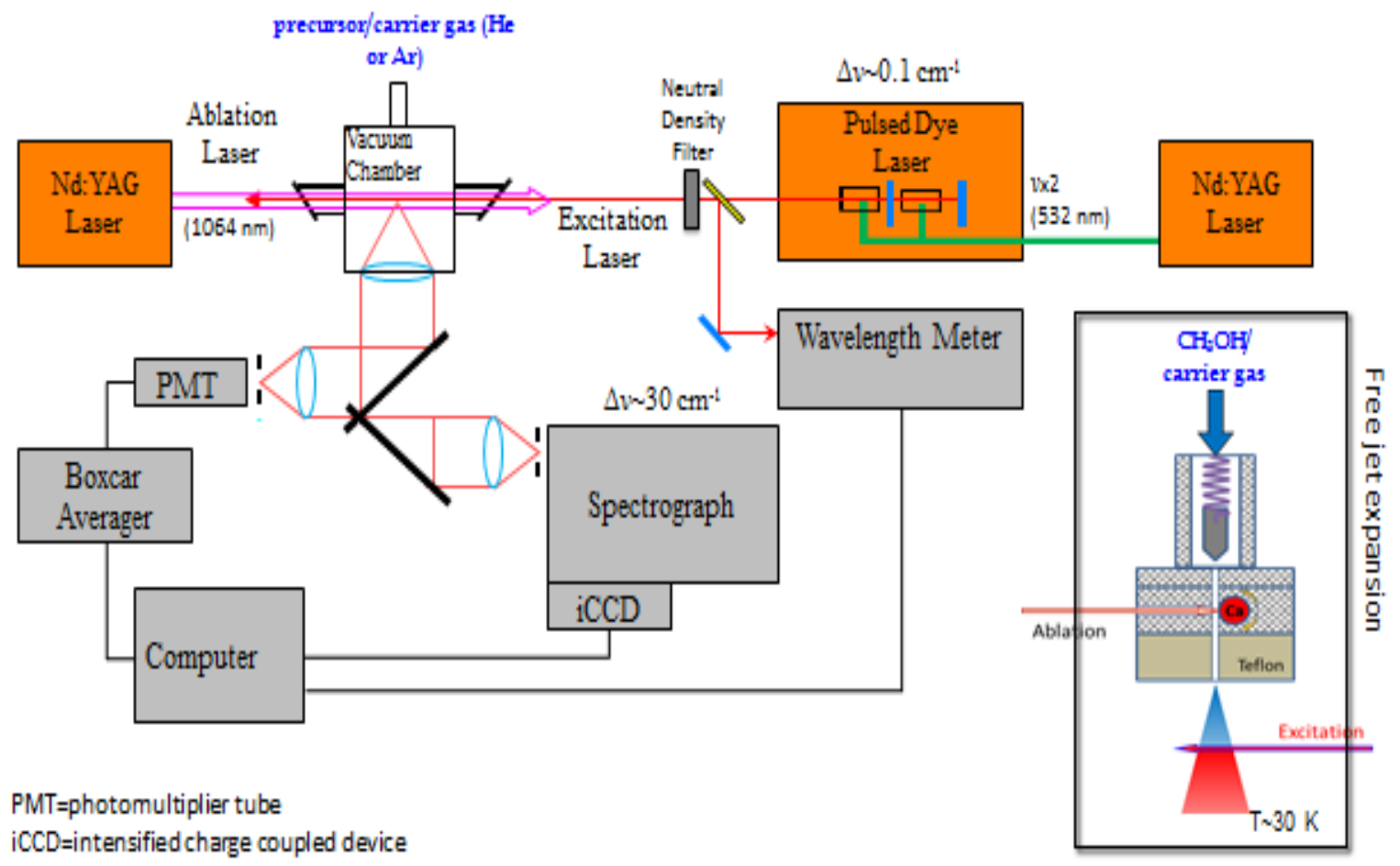

Figure 2.9: schematic diagram of LIF/DF experiment 

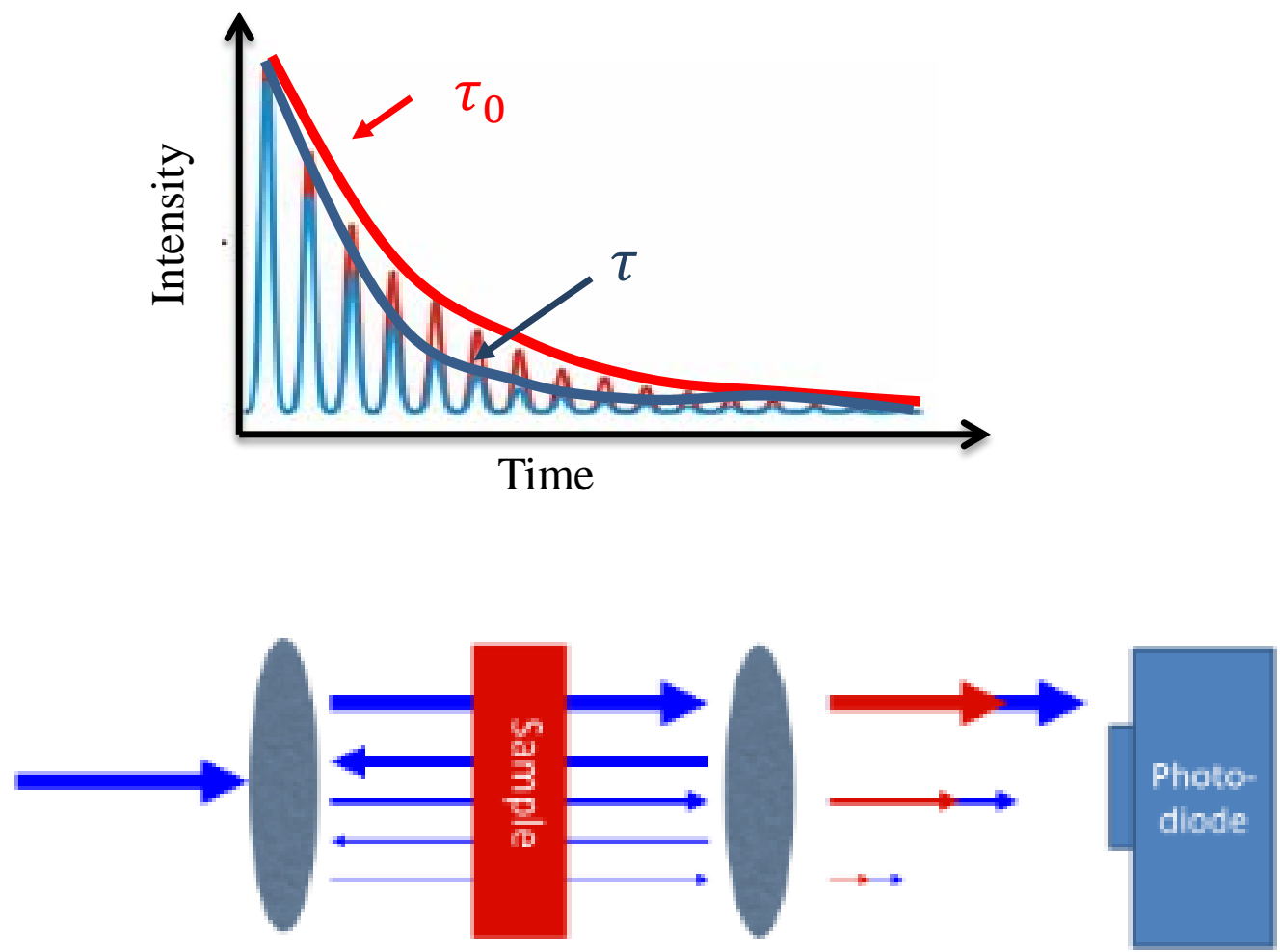

Figure 2.10: Principles of CRDS. Ring-down cavity is defined by two highly reflective mirrors and the sample is introduced into the cavity. Transmitted photons leaking from exiting mirror are indicated by blue arrows when the cavity is empty and by red arrows when it's filled with absorber. 


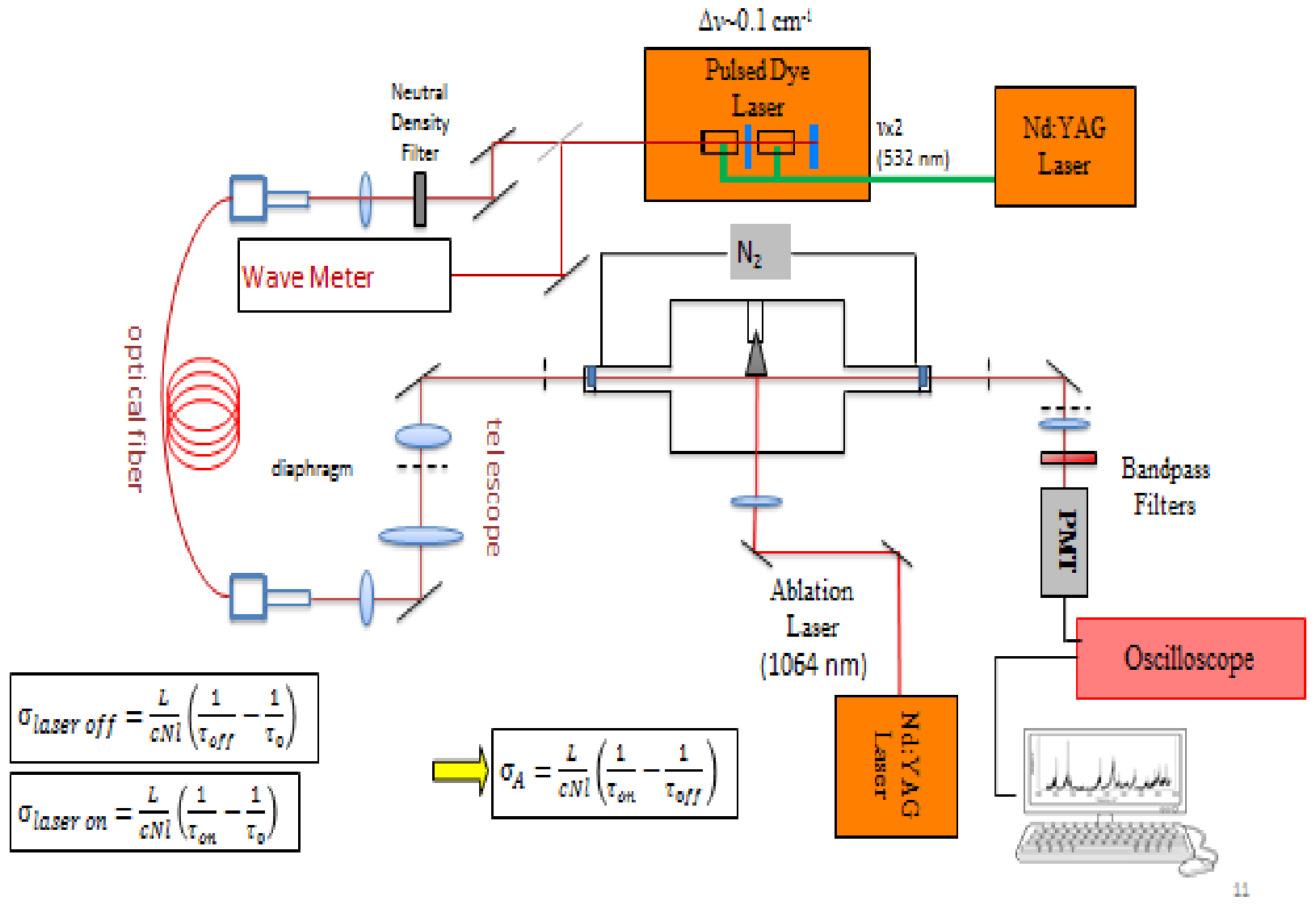

Figure 2.11: schematic diagram of cavity ring-down spectroscopy experiment 


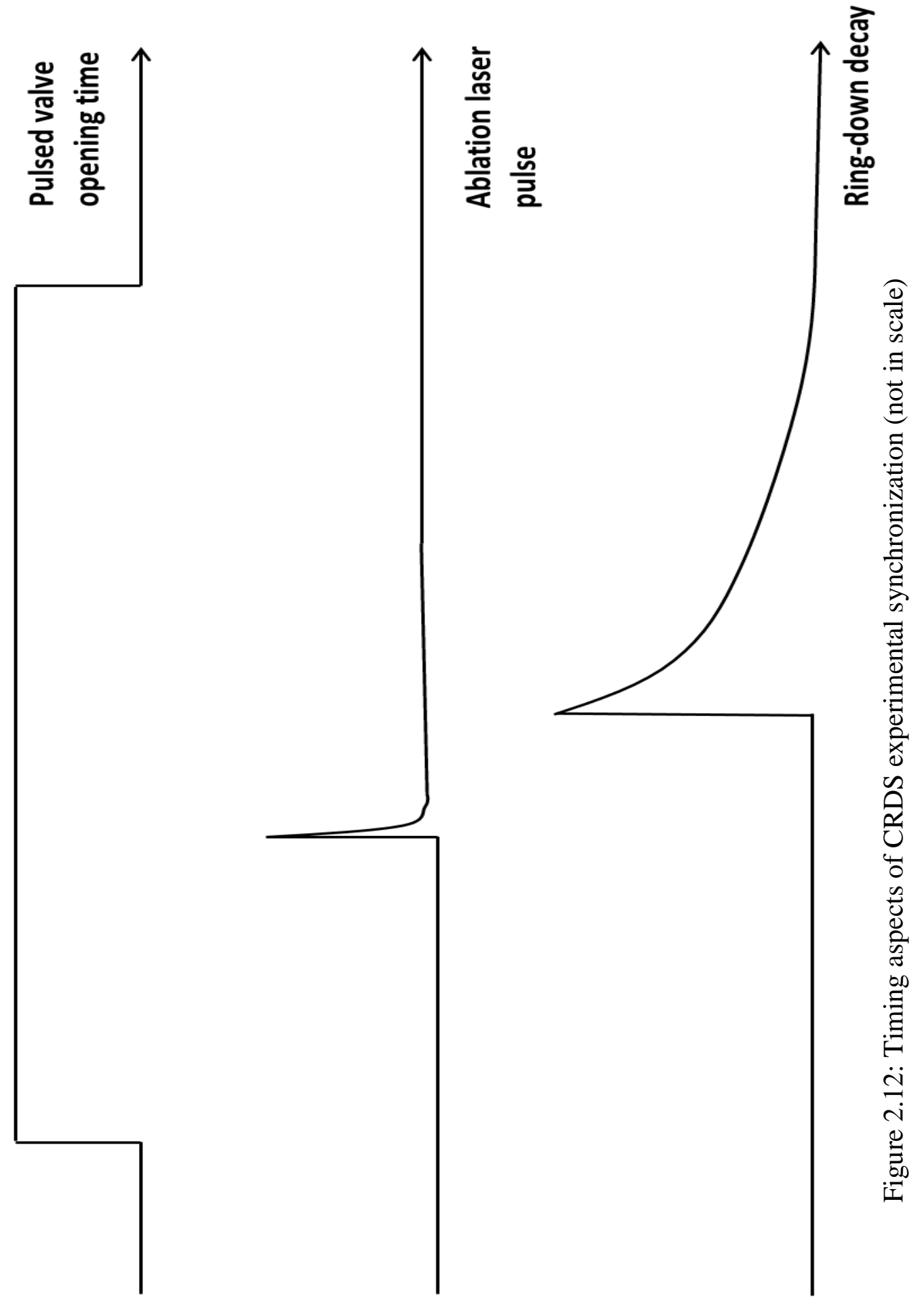




\section{CHAPTER III}

\section{LASER-INDUCED FLUORESCENCE AND DISPERSED- FLUORESCENCE SPECTROSCOPY OF JET-COOLED}

\section{CALCIUM METHOXIDE}

Metal-containing free radicals are essential intermediates in metal-molecule reactions, which are of significance at both the fundamental and applied levels. Studies on the mechanism of these reactions and the roles of the free radicals that they involve enhance our understanding of catalytic properties of metals, especially in bond activation. Specifically, there have been a large number of laser spectroscopy investigations of monovalent derivatives of the IIA and IIB group metals in the gas phase, which revealed the nature of molecular bonding and electronic structure of these metal-containing radicals. Typically, they are produced in Broida ovens ${ }^{28}$ or under supersonic-jet- cooled conditions, ${ }^{2}$, 29, 66, 67, and interrogated with laser-induced fluorescence (LIF) as well as dispersed fluorescence (DF) spectroscopy.

Recently, alkaline earth monoalkyl (MR) and monoalkoxide (MOR) free radicals, e.g., $\mathrm{CaCH}_{3}, \mathrm{CaOCH}_{3}, \mathrm{SrCH}_{3}$, and $\mathrm{SrOCH}_{3}$, have been proposed as candidates for laser cooling of polyatomic molecules ${ }^{4,68}$. These molecules can be regarded as methyl substitutions of metal monohydrides (MH) and hydroxides (MOH), respectively. Generally 
speaking, the criteria for selecting candidate molecules and transitions for direct laser cooling include ${ }^{69,70}$ : (1) existence of strong vibronic transitions to ensure a sufficient rate of photon scattering for rapid laser cooling, (2) availability and convenience of lasers to excite such transitions. (3) absence of spontaneous emission to intermediate electronic states that may terminate the cooling cycle, and (4) within the Born-Oppenheimer approximation a highly diagonal Franck-Condon (FC) matrix for vibronic transitions between involved electronic states to avoid population leakage. .Electronic transitions between the ground $(\tilde{X})$ and the two lowest-energy electronic excited $(\tilde{A}$ and $\tilde{B})$ states of MOR radicals possess high oscillator strength, thanks to their atomic-like nature. They lie in the visible or near-IR spectral region that can be covered by commercially available external cavity diode lasers. The $\tilde{A}$ state is particularly suitable for laser cooling because of the absence of intermediate electronic states between the $\tilde{A}$ and $\tilde{X}$ states. The last of the criteria for direct laser cooling of molecules involves the FC matrix. For MR and MOR free radicals, transitions to lowestenergy electronic excited states correspond to electron promotion to a nearly non-bonding orbital, a fact that suggests the existence of semi-closed transition loops for laser cooling. FC factors for the $\tilde{A}-\tilde{X}$ the transition of MOR, as well as MR radicals with $\mathrm{M}=\mathrm{Sr}$ and Ca, have been calculated $a b$ initio $^{68}$. It was predicted that the FC matrices are essentially diagonal for low vibrational energy levels. The origin $\left(v^{\prime}=0 \leftarrow v^{\prime \prime}=0\right)$ transition can therefore be used for laser cooling. Surprisingly, experimental information related to FC factors of these molecules is scarce. To the best of our knowledge, our previous DF measurement of the calcium ethoxide $\left(\mathrm{CaOC}_{2} \mathrm{H}_{5}\right)$ radical provides the only experimental vibronic spectrum of alkaline earth monoalkoxide radicals produced under jet-cooled conditions ${ }^{3}$, although rotationally resolved LIF spectra of their $\tilde{A}^{2} E-\tilde{X}^{2} A_{1}$ transition have been reported by 
different authors ${ }^{48,71}$ including a recent investigation ${ }^{71}$ of $\mathrm{CaOH}$ and $\mathrm{CaOCH}_{3}$ that also was motivated by an interest in their laser cooling. Recently we recorded LIF and DF spectra of a series of calcium monoalkoxide radicals. The present chapter reports experimental and computational studies on the smallest of them, namely, the calcium methoxide $\left(\mathrm{CaOCH}_{3}\right)$ radical.

For molecules the size of $\mathrm{CaOCH}_{3}$ or smaller, one would expect that quantum chemical calculations should provide a very useful guide for understanding the line positions and intensities in their spectra, particularly $\tilde{A}-\tilde{X}$ transition in $\mathrm{CaOCH}_{3}$. Of course, quantum chemical calculation almost always assumes the Born-Oppenheimer approximation (BOA), which separates electronic and nuclear motion. The degree of validity of BOA is open to question for open-shell, polyatomic molecules like $\mathrm{CaOCH}_{3}$, for more than one reason. Metal atoms give rise to moderately close-lying electronic states and symmetry, as occurs in $\mathrm{CaOCH}_{3}$, produces degenerate states. In addition, the quantum chemical calculations almost always ignore spin-orbit (SO) coupling, which, if treated at all, is done in a phenomenological way. $\mathrm{CaOCH}_{3}$ is a good molecule to investigate these approximations, as their breakdown in this molecule should be modest. Certainly the breakdown will become more pronounced for heavier metal atoms. Therefore the understanding of the degree of BOA breakdown and its ramifications is an important topic to be probed.

Nominally, $\mathrm{CaOCH}_{3}$, as well as other metal mono-methoxides, belong to the $C_{3 v}$ point group. However, their first electronic excited $\left(\tilde{A}^{2} E\right)$ state orbitally degenerate and hence subject to the Jahn-Teller (JT) vibronic interactions which, if sufficiently strong, could distort the equilibrium geometry and lower the molecular symmetry to $C_{s}$. As 
demonstrated later in the present paper, the $\mathrm{JT}$ effect in $\mathrm{CaOCH}_{3}$ has both linear and quadratic contributions, with the latter analogous to the Renner-Teller (RT) effect in $\tilde{A}^{2} \Pi$ state $\mathrm{CaOH}$. Molecular constants determined from the previous analysis ${ }^{71-73}$ of the rotationally resolved LIF spectra $\tilde{A}^{2} E-\tilde{X}^{2} A_{1}$ origin band suggest that the JT effect in the $\tilde{A}^{2} E$ state is weak and the molecules retains $C_{3 v}$ symmetry in this state but further investigation is warranted to see if there exist any observable spectral effects. The $\tilde{A}^{2} E$ state also interacts with the nearby $\widetilde{B}_{2} A_{1}$ state via the pseudo-Jahn-Teller (pJT) coupling, which is analogous to vibronic interaction between the $\tilde{A}^{2} \Pi$ and the $\tilde{B}^{2} \Sigma^{+}$states of the linear triatomic $\mathrm{CaOH}$ radical $^{74,75}$. The $\mathrm{pJT}$ effect is expected to alter the vibrational structure of the $\tilde{A}^{2} E$ state and affect the vibronic spectra of the $\tilde{A}^{2} E-\tilde{X}^{2} A_{1}$ transition. Of course, the SO interaction splits the $\tilde{A}^{2} E$ state into two SO components, further complicating the vibronic spectra. As for $\mathrm{CaOH}$, the $\mathrm{SO}$ constant of $\mathrm{CaOCH}_{3}$ is positive, which implies that the upper SO component of the $\tilde{A}$ the state is an $\mathrm{E}_{3 / 2}$ state, while the lower one is an $E_{1 / 2}$ state. Due to the similarity between the spin-vibronic structures of $\mathrm{CaOH}$ and $\mathrm{CaOCH}_{3}$, the latter may be referred to as a "pseudo-linear molecule" when molecular rotation is omitted. The present work includes both experimental and computational investigations on $\mathrm{CaOCH}_{3}$. First, we report LIF and DF spectra of the $\tilde{A}^{2} E-\tilde{X}^{2} A_{1}$ transition, from which vibrational frequencies and intensities can be determined. Second, quantum chemical calculations using complete active space selfconsistent field (CASSCF) and coupled-cluster singles and doubles (CCSD) methods have been carried out. The computational work yields harmonic frequencies of vibrational modes of both the $\tilde{A}^{2} E$ and $\tilde{X}^{2} A_{1}$ states. In addition, FC factors and vibrational branching ratios (VBRs) for vibronic transitions between these two electronic states were computed. It is 
important to note that for the emission spectroscopy, VBRs are determined by not only FC factors but also the transition frequencies $(v)$ because the Einstein A coefficient is proportional to $v^{3}$. Experimentally, VBRs correspond to normalized relative intensities of different vibronic bands in the DF spectra. Comparison between the experimental spectra and simulations using the calculated vibrational frequencies and intensities allows the assignment of most observed vibronic transitions. However, there exist experimentally observed transitions that are not predicted in the simulation. In particular, transitions to the CaOC bending $\left(v_{8}\right)$ levels, a JT and pJT-active mode, are symmetry-forbidden under BOA but were observed in both LIF and DF spectra in the present work. Therefore, further calculations have been undertaken to try to explain these additional transitions by including the effect of vibronic coupling and SO interactions.

CaOC bending levels have special significance in the search for the permanent electric dipole moment of the electron (eEDM) and time-reversal-symmetry-violating (TRSV) interactions. As proposed by Kozyryev1 and Hutzler ${ }^{76}$, degenerate vibrational and rotational motions of linear molecules (e.g., $\mathrm{CaOH}$ ) and pseudo-linear molecules (e.g., $\left.\mathrm{CaOCH}_{3}\right)$ provide energy-level doublets that enable full polarization and internal comagnetometers. This allows the observation of the reversal of the EDM interaction without changing any lab fields and hence provides crucial robustness to systematic effects. In particular, vibrational states of the $\mathrm{CaOH}$ or $\mathrm{CaOC}$ bending mode with opposite parties related to the vibrational angular moment $(l)$ can be easily polarized in electric fields. Highprecision measurements of energy level structure of these states and transition intensities are therefore critical to future laser cooling of $\mathrm{CaOCH}_{3}$ and similar linear and "pseudo- linear" molecules and detection of TRSV interactions. In spite of the lack of rotational resolution, 
the present paper reports the first step toward quantitative understanding of energy level structure of these TRS-related vibrational states and transitions in which they are involved.

\subsubsection{Experimental (LIF and DF)}

Our LIF/DF apparatus for the study of jet-cooled metal-containing free radicals produced with laser ablation has been described in a previous publication ${ }^{3}$. Recently, a new laser-ablation nozzle has been built and employed for efficient, stable, and enduring production of metal-containing molecules. The laser-ablation nozzle features a motorized stage that can drive simultaneously the rotational and translational motions of a metal rod ${ }^{57}$. The continuous motion of the metal rod ensures that each laser shot ablates a fresh spot on its surface and hence the stability of the metal vapor. In the present work, $\mathrm{CaOCH}_{3}$ radicals were produced by $1064 \mathrm{~nm}$ laser ablation of a calcium rod (ESPI metals) in the presence of methanol (Sigma-Aldrich) under jet-cooled conditions. Fundamental output of an Nd:YAG laser (Continuum, Powerlite Precision II 8000) was used as the ablation source. Both Helium and Argon (150 psi above atmosphere) were used as carrier gases. Approximately twice as large fluorescence signal was obtained using Argon, compared to He; however, the Helium carrier gas provides the lower vibrational temperature. The results presented in this paper were obtained using only Helium because of the relative absence of hot bands. The seeded flow expanded through a pinhole valve $(0.3 \mathrm{~mm}$ in diameter) into the vacuum chamber (stagnation pressure=20 millitorr). A $12 \mathrm{~mm}$ thick Teflon extension with a $1.5 \mathrm{~mm}$ diameter orifice at the center was attached to the supersonic nozzle for additional vibrational cooling ${ }^{77}$. A dye laser (Spectra-Physics, Cobra Stretch) pumped by 
the second harmonic of an Nd:YAG laser (Spectra-Physics GCR-4) output was used to excite the $\tilde{A}^{2} E-\tilde{X}^{2} A_{1}$ transition of $\mathrm{CaOCH}_{3}$. For the LIF experiment, fluorescence was collected by a lens system perpendicular to both the excitation laser beam and the jet expansion and focused onto a photomultiplier tube (PMT, Hamamatsu, H10721- 01). The spectral linewidth of the LIF spectra is $\sim 0.1 \mathrm{~cm}^{-1}$, limited mainly by the linewidth of the pulsed dye laser ( $\Delta v=\sim 0.06 \mathrm{~cm}^{-1}$ ) and the residual Doppler broadening. The frequency of the pulsed dye laser was calibrated by a wavemeter (HighFinesse, W7). Because the rotational structure is not resolved (rotational temperature $25 \mathrm{~K}$ ), the accuracy of the vibronic transition frequencies in the LIF spectrum is limited by the width of the rotational contour and estimated to be $\sim 1 \mathrm{~cm}^{-1}$.

For the DF experiment, fluorescence is focused into and dispersed by a monochromator (Acton Research, SpectraPro 300i) equipped with an intensified CCD (iCCD) camera (Princeton Instruments, PI-MAX 512). A typical spectral resolution of $\sim 20 \mathrm{~cm}^{-1}$ was observed for DF spectra. The wavelength of the monochromator was calibrated using a mercury arc lamp. The frequency uncertainty of the DF spectra is limited by the resolution of the monochromator to $\sim 5 \mathrm{~cm}^{-1}$.

The intensities of the recorded LIF spectra were calibrated by the wavelength-dependent sensitivity of the PMT. Intensities of the DF spectra were calibrated by the grating efficiency and the quantum efficiency of the iCCD camera, both of which are wavelength dependent. Wavelength- dependencies of the PMT sensitivity, the grating efficiency, and the quantum efficiency of the iCCD camera are taken from specifications without experimental verification, which might introduce some error in the FC factors and VBRs. However, such errors are expected to be small because of the weak wavelength-dependence of the aforementioned quantities in the relatively narrow wavelength range covered in the 
present work. The experimental intensities for the $\tilde{A}^{2} E \leftarrow \tilde{X}^{2} A_{1}\left(\tilde{A}^{2} E \rightarrow \tilde{X}^{2} A_{1}\right)$ vibronic transitions are determined using the integrated intensities of the vibronic bands in the LIF (DF) spectra.

A technical challenge in determining emission transition intensities is that dispersed fluorescence signal due to transitions to the ground vibrational level of the ground electronic state is contaminated by scattering of the LIF excitation laser because they have the same wavelength, i.e., the red-shift is zero for these emission transitions. In addition, the iCCD camera demonstrates nonlinear response with high fluorescence intensities ${ }^{62}$, for instance, for the origin band emission. Experimentally the measured intensity for the origin band transitions are therefore likelynot particularly accurate.

Several measures have been taken in an effort to improve the precision of experimental intensities in the excitation spectrum. For example, the LIF excitation laser was attenuated to $\sim 10 \mathrm{~mJ} / \mathrm{cm}^{2}$ to minimize the power-saturation of transitions. Nonetheless, we decided to perform separate pulsed-laser cavity ring-down (CRD) spectroscopy measurements of the $\tilde{A}^{2} E \leftarrow \tilde{X}^{2} A_{1} 0_{0}^{0}$ and $4_{0}^{1}$ transitions to calibrate the LIF measurements. The CRD apparatus employed the same dye laser source as the LIF but with significantly lower pulse energy $(\sim 400 \mu \mathrm{J})$. Details of the jet-cooled CRD apparatus ${ }^{78,79}$ are presented in Section 3.2.2. Thanks to the weak intra-cavity laser field and because re-emission to the ground electronic state ( $\sim 50 \mathrm{~ns})$ are significantly faster than the ring-down time ( $\sim 7 \mu$ st the peaks of the original band), power-saturation is negligible in pulsed-laser CRD spectroscopy. Comparing the LIF and CRD spectra confirms that the origin $\left(0_{0}^{0}\right)$ transitions were saturated in the LIF experiment even with the lowest laser powers. Comparing the ratio of the integrated intensities of $0_{0}^{0}$ and $4_{0}^{1}$ bands in the CRD and LIF spectra 
(Figure 3) allows a scaling factor, $S_{F}=10.75 \pm 0.66$ to be determined for the ratio of the bands' intensities. It is important to consider in some detail the uncertainty assigned to $S_{F}$, as it is the dominating error for the $0_{0}^{0}$ and $4_{0}^{1}$ band intensities. The assigned uncertainty in $S_{F}$ represents the random error in measuring the intensities of the ratio of the $0_{0}^{0}$ and $4_{0}^{1}$ bands in the CRD spectra. For the other bands error arises from two principal sources: (i) the $\mathrm{S} / \mathrm{N}$ in the spectra which is higher in CRD than in the LIF spectrum and (ii) intensity fluctuations in the $\mathrm{CaOCH}_{3}$ concentrations due to pulse-to-pulse fluctuations of the ablation laser power and its efficiency of ablation at different locations on the rotating Ca rod. The $\mathrm{S} / \mathrm{N}$ limitation $(\approx 0.002$ of the normalized LIF intensity, $\approx 0.005$ for CRD) is independent of transition intensity. The concentration fluctuations $(\approx 5 \%)$ are proportional to the transition's intensity.

Beside random errors in the CRD experiments, one must consider the existence of possible systematic errors. We can identify two possible systematic errors that might make non-negligible contributions. One is the assumption of no power saturation of the transition in the excitation spectrum. In principle, the degree of saturation could be determined by measuring the $3_{0}^{1}$ in the CRD experiments. However, we found that $3_{0}^{1}$ intensity transition was too weak to observe, which, given the $\mathrm{S} / \mathrm{N}$ on $4_{0}^{1}$, would imply an upper limit for $S_{F}$ of ,:S 2 times the value above.

Finally, it is known that in CRD spectroscopy multi-exponential ring-down can occur which limits the measured intensities of the strongest lines to less than their true values. This process is discussed in some detail in an early work ${ }^{80}$ which shows that in the limiting case of an infinitely narrow laser, these effects vanish and can be minimized when using a finite laser linewidth by considering only the early part of the ring-down curve. 
Our experimental protocol samples only the first $1 / 3$ of the ring-down curve, i.e., the faster component. Using the plots in Ref. ${ }^{79}$, we conclude that with our laser bandwidth and sample absorption an upper limit can be estimated for an increase of $S_{F}$ by a factor of ,:S 2 . The overall uncertainty of the integrated transition intensity of an individual vibronic band is calculated as the sum in quadrature of error sources listed above. Uncertainties of experimentally determined FC factors and VBRs are calculated from normalized transition intensities and their relative errors using standard error propagation analysis. (See Section 4.2 of chapter 4 for more details.) 


\subsubsection{Experimental (cavity ring-down spectroscopy)}

The CRD apparatus used in the present work is similar to the room-temperature CRD setup described in our previous papers ${ }^{62,78}$, except that the free radicals are produced by laser ablation under jet-cooled conditions as described in the main text. CRD mirrors (Los Gatos Research, $R>99.995 \%$, center wavelength=620 nm) were mounted on two arms of the vacuum chamber to form a ring-down cavity with a length of $L=76 \mathrm{~cm}$. The ring-down mirrors were purged by an $\mathrm{N}_{2}$ flow continuously to prevent contamination (mainly by the metal vapor). Transmission of the ring-down laser beam (Sirah, Precision Scan) through the cavity was focused onto a photomultiplier tube (PMT, Hamamatsu, H10721-01). The signal was acquired with an oscilloscope (Tektronix, DPO3014). The empty-cavity ring-down time $\left(\tau_{0}\right)$ was about $50 \mu \mathrm{s}$. Typically, the ring-down curves were averaged 64 times on the oscilloscope at each laser wavelength before being sent to a PC via a USB port. The averaged ring-down curves of up to 100,000 data points were fit to a biexponential decay function. The longer of the two fit time constants was used to calculate the absorption (in ppm/pass). Ring-down signal was collected at each wavelength with the ablation laser on and off. The "ablation laser off" CRD spectrum is subtracted from the “ablation laser on” spectrum to obtain the net spectrum of radicals. The ablation and ringdown laser pulses, the supersonic expansion nozzle, data acquisition and processing, and wavelength scan of the ring-down laser are all controlled by a home-made LabVIEW

program and synchronized by a digital pulse and delay generator (Stanford Research Systems, DG645) at a repetition rate of $20 \mathrm{~Hz}$. 


\subsection{Quantum chemical calculations}

In the present work, the ground $\left(\tilde{X}^{2} A_{1}\right)$ and the first excited $\left(\tilde{A}^{2} E\right)$ electronic states of $\mathrm{CaOCH}^{3}$ were calculated using CASSCF and CCSD methods with the cc-pVTZ basis set. CASSCF calculations were performed with the Gaussian 09 program package ${ }^{80}$, while CCSD calculations with the CFour software ${ }^{81}$.

The $\tilde{A}^{2} E-\tilde{X}^{2} A_{1}$ the electronic transition of $\mathrm{CaOCH}_{3}$ corresponds to the promotion of the unpaired electron in the $4 s$ orbital of $\mathrm{Ca}^{+}$to its $4 p \sigma\left(p_{x}\right.$ or $\left.p_{y}\right)$ orbitals weakly perturbed by the presence of a methoxy group ${ }^{82,83}$. The active space used in the CASSCF calculation, therefore, contains 3 electrons in 6 molecular orbitals (MOs). The chosen MOs are characterized by the $3 s, 4 s, 3 d_{z}^{2}, 4 p_{x}, 4 p_{y}$, and $4 p_{z}$ orbitals of the calcium cation, although the first one is mixed with the $2 s$ orbital of the oxygen atom (Figure 1). Of these six MOs, the first one (Ca 3s) is doubly occupied in the ground electronic state, the second one (Ca $4 s)$ is singly occupied, and the rest are virtual orbitals. The properties of $\tilde{A}^{2} E$ state are calculated by averaging over the two degenerate components of the electronic state with equal weights and restricting the molecule to $C_{3 v}$ symmetry.

Singles and doubles coupled cluster treatment using a cc-pVTZ basis set was employed for the $\tilde{X}^{2} A_{1}$ state. The JT interaction in the excited state $\tilde{A}^{2} E$ and the pJT coupling between the $\tilde{A}^{2} E$ and $\tilde{B}^{2} A_{1}$ states are treated using the equation of motion electron excitation coupled-cluster theory (EOMEE-CCSD/cc-pVTZ). The two target degenerate components of the $\tilde{A}^{2} E$ state is accessed using the same method as described in the

literature (c.f. Refs. ${ }^{83-85}$ ). $C_{s}$ groups (an abelian subgroup of the $C_{3 v}$ group) are used as they transform as ${ }^{2} A^{\prime}$ and ${ }^{2} A^{\prime \prime}$. This method has been used for studying JT active and vibronic 
coupling cases for a number of molecules. The reference state for the EOMEE-CCSD calculation is the ground state of $\mathrm{CaOCH}_{3}$.

Geometry optimization of the $\tilde{X}^{2} A_{1}$ and $\tilde{A}^{2} E$ states provide equilibrium bond lengths and bond angles, as well as rotational constants, of both states that are summarized in Table 1 . Since the $\tilde{A}^{2} E$ state is orbitally doubly degenerate and subject to the JT effect, a state averaged calculation with equal weights was carried out for its geometry optimization, which determines the minimum of the conical intersection (CI) seam. Geometric parameters of the $\tilde{A}^{2} E$ state in Table 1 are therefore for the CI geometry that belongs to the $C_{3 v}$ symmetry. The largest change in geometry upon the $\tilde{A}^{2} E \leftarrow \tilde{X}^{2} A_{1}$ excitation is in the $\mathrm{CaO}$ bond length $\left(\Delta_{\mathrm{rCaO}} \sim 30 \mathrm{~m} \AA\right)$. Therefore, one expects vibronic transitions to the $\mathrm{CaO}$ stretch levels in the LIF/DF spectra, to be the strongest non-diagonal transitions.

Compared to previous experimental values, both CASSCF and CCSD calculations predict the $\mathrm{CH}$ bond and $\mathrm{OCH}$ angle of the $\tilde{X}^{2} A_{1}$ and $\tilde{A}^{2} E$ states accurately. The calculated $\mathrm{CaO}$ and $\mathrm{CO}$ bond lengths are less accurate. However, the calculated changes in the bond lengths upon the electronic excitation are close to experimentally determined values.

Vibrational assignment of LIF and DF spectra in the present work is guided by the quantum chemical calculations (see Section 3.3.1 and Section 3.3.2). $\mathrm{CaOCH}_{3}$ has 8 vibrational modes, including 4 " $\mathrm{a}_{1}$ "'modes and 4 doubly degenerate "e" modes. Harmonic frequencies of both the $\tilde{X}^{2} A_{1}$ and the $\tilde{A}^{2} E$ state vibrational modes are listed in Table 2. Calculation of FC factors for both absorption and emission transitions under the harmonic oscillator approximation was performed using the ezSpectrum software ${ }^{84}$ The LIF 
spectrum of the $\tilde{A}^{2} E \leftarrow \tilde{X}^{2} A_{1}$ transition with $\mathrm{v}^{\prime \prime}=0$ and the DF spectrum of the $\tilde{A}^{2} E \leftarrow$ $\tilde{X}^{2} A_{1}$ transition with $\mathrm{v}^{\prime}=0$ were simulated using the calculated vibrational frequencies and FC factors. In simulating the LIF spectrum, the vibrational temperature is set to $50 \mathrm{~K}$. The Gaussian line shape with a full-width at half-maximum (FWHM) of $5 \mathrm{~cm}^{-1}$ and $25 \mathrm{~cm}^{-1}$ was used to generate the simulated LIF and DF spectra, respectively.

A diabatic spin-vibronic model was employed to simulate the spectral properties of forbidden transitions. JT coupling within the $\tilde{A}$ state was calculated by PES scanning along with the JT active modes using the CFour software. The pJT coupling is calculated using the method described by Ichino et.al ${ }^{85}$. The numerical values of these couplings are presented in chapter-4. Spin vibronic eigenvectors were computed for the $\tilde{A}$ state using SOCJT2 ${ }^{86,87}$, and vibronic transition intensities were calculated as described in chapter 4 . 


\subsection{Results}

\subsubsection{LIF Spectrum}

The experimental LIF spectrum of the $\tilde{A}^{2} E \leftarrow \tilde{X}^{2} A_{1}$ the transition of $\mathrm{CaOCH}_{3}$ (Figure 2c) features six doublets with a frequency interval of $\sim 67 \mathrm{~cm}^{-1}$ between each pair of peaks. The frequency interval is consistent with the SO splitting of the vibrational ground level of the $\tilde{A}^{2} E$ state determined in fitting the rotationally resolved $\tilde{A}^{2} E \leftarrow \tilde{X}^{2} A_{1}$ origin band in the LIF spectrum of $\mathrm{CaOCH}_{3}$. By comparison, the SO splitting of all accessed vibronic levels of the $\tilde{A}^{2} \Pi$-state $\mathrm{CaOH}$ radical is also $\sim 67 \mathrm{~cm}^{-1}$. The observation that $\mathrm{CaOH}\left(\tilde{A}^{2} \Pi\right)$ and $\mathrm{CaOCH} 3\left(\tilde{A}^{2} E\right)$ have essentially identical SO splitting again suggests that the methyl substitution has minimal influence on the electronic structure of the molecule and the vibronic interaction scheme in the $\mathrm{CaOCH} 3$ radical is similar to that of $\mathrm{CaOH}$.

The strongest doublet centered at $\sim 15920 \mathrm{~cm}^{-1}$, is assigned to the origin transition, with the higher and lower frequency peaks assigned to the $\tilde{A}^{2} E_{3 / 2} \leftarrow \tilde{X}^{2} A_{1}$ and the $\tilde{A}^{2} E_{1 / 2} \leftarrow \tilde{X}^{2} A_{1}$ transitions, respectively. By comparing to the simulation in terms of both vibrational frequencies and transition intensities, doublets centered at 502, 1156, and $1387 \mathrm{~cm}^{-1}$ to the blue of the origin band can be assigned to transitions to the $v^{\prime}=1$ levels of the $\mathrm{CaO}$ stretch mode $\left(4_{0}^{1}\right)$, the asymmetric $\mathrm{CaOC}$ stretch mode $\left(3^{1}\right)$, and the $\mathrm{CH}_{3}$ umbrella mode $\left(2^{1}\right)$, respectively. The remaining strong doublet, centered at $1001 \mathrm{~cm}^{-1}$, is assigned to a transition to the $v^{\prime}=2$ level of the $\mathrm{CaO}$ stretch mode $\left(4_{0}^{2}\right)$. 
The weaker doublet observed centered at $149 \mathrm{~cm}^{-1}$ blue of the origin band, is not predicted by either the CCSD or CASSCF simulation. The most reasonable assignment of this doublet is the transition to the $\mathrm{v}^{\prime}=1$ level of the $\mathrm{CaOC}$ bending mode $\left(8_{0}^{1}\right)$, for which the CASSCF and CCSD calculations predict a harmonic frequency of 157 and $122 \mathrm{~cm}^{-1}$, respectively. The doublet results from the splitting of the vibrational transition into two SO levels similar to the other lines in the spectrum. Due to its vibrational symmetry, transitions between the $8^{1}$ level of the $\tilde{A}^{2} E$ state and the vibrationless ground level of the $\tilde{X}^{2} A_{1}$ state is forbidden. However, such transitions often gain intensity through vibronic interactions, which we will consider later. 


\subsubsection{DF spectra obtained by pumping the origin transitions}

Figure 4c illustrates the DF spectrum obtained by pumping the $\tilde{A}^{2} E_{1 / 2} \leftarrow \tilde{X}^{2} A_{1}$ the transition of CaOCH3. In addition to the strong origin band, all observed peaks can be assigned to transitions to the $v^{\prime \prime}=1$ levels of the $\mathrm{CaO}$ stretch mode $\left(v_{4}\right)$, asymmetric $\mathrm{CaOC}$ stretch $\left(v_{3}\right)$, CH3 umbrella $\left(v_{2}\right)$, or the overtone and combination levels of these modes (See Figure 3.4c for assignment.) These transitions are predicted in the simulated DF spectra. The peak at $1450 \mathrm{~cm}^{-1}$ is assigned to the $2_{1}^{0}$ transition. It may also be assigned to the $4_{3}^{0}$ transition if only the vibrational frequency is considered. However, intensity simulation supports the present assignment. The DF spectrum obtained by pumping the $\tilde{A}^{2} E_{3 / 2} \leftarrow$ $\tilde{X}^{2} A_{1}$ transition (Figure 3.4d) also contains the aforementioned peaks. However, it has additional spectral features. First, the strongest peaks $\left(0_{0}^{0}, 4_{1}^{0}, 3_{1}^{0}\right)$ that are present in the DF spectrum obtained when pumping the $\tilde{A}^{2} E_{1 / 2} \leftarrow \tilde{X}^{2} A_{1}$ LIF transition is all accompanied by a weaker "child peak" to the red in the DF spectrum. The separation between the parent and child peaks is $\sim 67 \mathrm{~cm}^{-1}$, identical to the SO splitting of the vibronic levels of the $\tilde{A}^{2} E$ state. The presence of these "child peaks" is therefore ascribed to collision-induced population transfer from the upper $\tilde{A}^{2} E_{3 / 2}$ SO level to the lower $\tilde{A}^{2} E_{1 / 2}$ SO level ${ }^{88}$. The subsequent $\tilde{A}^{2} E_{1 / 2} \leftarrow \tilde{X}^{2} A_{1}$ transitions are red-shifted relative to their counterparts in the $\tilde{A}^{2} E_{3 / 2} \leftarrow \tilde{X}^{2} A_{1}$ transition, i.e., their "parent peaks", and the magnitude of the red-shift is equal to the SO splitting of the $\tilde{A}^{2} E$ state. Second, the DF spectrum obtained by pumping the $\tilde{A}^{2} E_{3 / 2} \leftarrow \tilde{X}^{2} A_{1}$ origin transition contains an extra peak at $278 \mathrm{~cm}^{-1}$ red-shift. This peak is absent in the DF spectrum obtained by pumping the $\tilde{A}^{2} E_{1 / 2} \leftarrow \tilde{X}^{2} A_{1}$ origin transition. 
The position of this peak matches that expected for the $v^{\prime \prime}{ }_{8}=2$ level of the $\mathrm{CaOC}$ bending mode in the simulated DF spectrum. Possible explanations for the presence of this peak, only seen when pumping the $\tilde{A}^{2} E_{3 / 2}$ origin is discussed below. As stated in Section 3.2, direct experimental determination of the intensity of the origin transition from the DF spectra is difficult due to contamination from the scattering of the excitation laser. It is therefore fixed to the value determined in the CRD experiments. Relative intensities of other transitions in emission are determined by the maintenance of their experimentally determined intensity ratios in DF, while keeping the sum of the VBRs normalized. 


\subsubsection{DF spectra obtained by pumping other vibronic bands}

DF spectra obtained by pumping strong vibronic bands in the LIF spectrum, namely, $8_{0}^{1}, 4_{0}^{1}, 4_{0}^{2}$, and $3_{0}^{1}$, are illustrated in Figure 5b-e. Compared to those obtained by pumping the origin band (Figure 5a), DF transitions from a vibronic level of the $\tilde{A}^{2} E$ state to those $\tilde{X}^{2} A_{1}$-state vibrational levels of the pumped mode gain intensity thanks to larger FC factors. Additionally, transitions to combination levels of the pumped mode and other modes may also gain intensities because of the Duschinsky mixing ${ }^{88,89}$. A simple technique that reveals this relation is to blue-shift DF spectra by the ground-state frequencies of the pumped modes. The resulting spectra are shown in Figure 6. As demonstrated there both the CaOC stretch $\left(v_{3}\right)$ and the (p)JT-active $\mathrm{CaOC}$ bending $\left(v_{8}\right)$ modes are mixed with the $\mathrm{CaO}$ stretch $\left(v_{4}\right)$ mode, which can be explained by the elongation of the $\mathrm{CaO}$ bond length upon the electronic excitation, and hence the large mixing between the $\mathrm{CaO}$ stretch and other modes. To a lesser extent, the $v_{3}$ and $v_{8}$ modes are also mixed because both involve the same atoms and the frequency of the $3^{1}$ the level is close to the $8^{8}$ level. Such mixing manifests itself in two features in the DF spectra. First, a weak transition to the $8_{1} 3_{1}$ level is observable in the DF spectra when the $8_{0}^{1}$ band is pumped (see Figure 5b). Another observation that can be attributed to the mixing between these two modes is the long $v "{ }_{8}$ progression in the DF spectra obtained by pumping the $3_{0}^{1}$ transition (see Figure 3.5e). Because of the $e$ symmetry of the $v_{8}$ mode, only transitions to the $8_{v^{\prime \prime}}$ levels with even $v^{\prime \prime}$ transitions are allowed, as they have $a_{1}$ components. 
When the $4_{0}^{1}$ (CaO stretch) band in the LIF spectrum is pumped, the DF spectrum contains, among others, a strong peak at $1143 \mathrm{~cm}^{-1}$, designated by an " $\mathrm{X}$ " in Figure 3.5c. This peak defies any reasonable assignment to transitions of $\mathrm{CaOCH}_{3}$ especially when one considers that it is absent in the DF spectra obtained by pumping the $4_{0}^{2}$ band (see Figure 5d). It is probably due to impurity. Similarly, another peak in the spectrum obtained by pumping the $4_{0}^{1}$ band, labeled " $\mathrm{Y}$ " in Figure 3.5c cannot be assigned either and also is attributed to impurity. 


\subsection{Discussion}

\subsubsection{Comparison of CASSCF and CCSD calculations for the $\widetilde{A}$ and $\widetilde{X}$}

\section{states with experiment}

The simulated spectra using both CASSCF and CCSD results reproduce a number of features of the experimentally recorded spectra quite well. The calculated vibrational frequencies are listed in Table 3.2 in comparison with the experimental values. In general, the CCSD calculation predicts both the $\tilde{A}^{2} E$ and $\tilde{X}^{2} A_{1}$-state vibrational frequencies somewhat more accurately than the CASSCF calculation, especially for the CaOC stretch $\left(v_{3}\right)$ mode. The CCSD calculation predicts the $\tilde{X}^{2} A_{1}-\tilde{A}^{2} E$ transition frequency with fortuitous accuracy: $15918 \mathrm{~cm}^{-1}$ compared to the experimental value of $15925 \mathrm{~cm}^{-1}$.

Both CASSCF and EOM-CCSD calculations predict that the harmonic frequencies of vibrational modes in the $\tilde{A}^{2} E$ and the $\tilde{X}^{2} A_{1}$ states are quite similar, suggesting similar PESs for these two states. Indeed, vibrational frequencies determined in the experimental LIF and DF spectra have verified the prediction. Consequently, the calculated FC matrix for the $\tilde{A}^{2} E-\tilde{X}^{2} A_{1}$ transition of $\mathrm{CaOCH}_{3}$ is highly diagonal. Experimental values for the line intensities determined in the LIF and DF spectra are listed in Table 3.3 and Table 3.4 in comparison with calculated ones. In general, the observed intensities of the strong transitions agree well with those predicted by both the CASSCF (calc. 1) and CCSD (calc. 2) calculations. In Table 3.4, both FC factors and normalized VBRs from calculations are listed. If one pumps the $0_{0}^{0}$ band of the $\tilde{A}$ state, roughly $90 \%$ of the spontaneous emission occurs via the $0_{0}^{0}$ band, with most of the remainder via the $4_{1}^{0}$ 
(mainly) and the $3_{1}^{0}$ bands. However, it should be noted that the weaker bands, putatively involving $v_{8}$, are predicted to have no intensity while lines are clearly observed in the spectrum.

\subsubsection{Corrections to the quantum chemical results}

The nominally “forbidden” bands observed in both the LIF and DF spectra are attributed to transitions to the $v_{8}$ levels. They deserve further comments because of their critical roles in the spin-vibronic analysis as well as in the proposed search for eEDM and TRSV interactions (see Section 3.1). A fundamental reason why these bands may not be predicted accurately by the quantum chemical calculations is that the calculations assume the BOA and the harmonic approximation, and neglect SO coupling. The clear implication is that the appearance of these bands results from the limitation of these approximations. Therefore their understanding should be pursued since open-shell polyatomic molecules containing heavier atoms are a place where these approximations might be expected to fail to an even greater extent. 


\subsubsection{Vibronic coupling within and between the $\widetilde{A}$ and $\widetilde{B}$ states}

Possible vibronic interactions which are not taken into account by the $a b$ initio calculations are briefly summarized here. These interactions conventionally have been attributed to either JT or pJT interactions. The JT interactions, which we consider first, occur within the $\tilde{A}$ electronic state and are sub-divided into linear and quadratic JT effects, depending on whether they arise from terms in the power series expansion of the potential that are linear or quadratic in the normal coordinates. There is one linear term for each of the four $e$ JT-active modes which include $v_{5}-v_{8}$. The quadratic terms can be segregated according to the normal coordinates involved and are typically referred to as (a) quadratic JT, if they involve only a single JT mode, (b) bilinear terms including a JT-active and a totally symmetric vibrational mode, and (c) cross-quadratic ones involving two different JTactive $e$ vibrational modes. To these terms, we add the phenomenological SO coupling term,

$a \| L z S z$, which is diagonal in our spin-vibronic $\tilde{A}$ state basis with values $a \| \Lambda \Sigma$, where $a \|$ is the SO constant along the 3-fold molecular symmetry axis, and $\Lambda$ and $\Sigma$ are the projections of the electronic orbital (L) and spin (S) angular momenta upon the same axis.

As is described in detail in the chapter 4, we construct the spin-vibronic Hamiltonian matrix o with our SOCJT2 software and obtain the spin-vibronic eigenvalues and eigenfunctions.

For the SOCJT2 calculation, the various JT coupling terms can be parameterized in terms of the derivatives of the adiabatic PESs with respect to the normal coordinates. These derivatives are determined numerically by scanning the PES using EOMEE-CCSD methods. The SO coupling constant is taken from the experimental value reported for $\mathrm{CaOH}$ or the measured intervals in the CaOCH3 LIF spectrum, which yield essentially equal results of $a / /=67 \mathrm{~cm}^{-1}$. 
Although technical and quantitative details of our spin-vibronic analysis are given in the chapter 4, it is beneficial to present here the overall structure of the energy levels of (non-rotating) $\mathrm{CaOCH}_{3}$ radical and describe how the JT and SO interactions alter the energy level structure and induce vibronic transitions that are forbidden under BOA (Figure3.7). In the absence of vibronic interactions (Figure 3.7a), the origin transition ( $v^{\prime}=0$ $\left.\leftarrow v^{\prime \prime}=0\right)$ of the $\tilde{A}^{2} E-\tilde{X}^{2} A_{1}$ system is allowed, while the $\left(v^{\prime}{ }_{8}=1 \leftarrow v^{\prime \prime}=0\right)$ transition is forbidden because of its vanishing FC constant. The linear (first-order) JT effect lowers the vibrationless ground $\left(v^{\prime}=0\right)$ level of the $\tilde{A}^{2} E$ state, and splits the $v^{\prime}{ }_{8}=1$ level into two vibronic levels (Figure 7b). The upper and lower components of the $v^{\prime}{ }_{8}=1$ vibronic level are of $e$ and $a_{1}+a_{2}$ symmetry, respectively. The quadratic (second-order) JT effect further lowers the $v^{\prime}=0$ level, but it remains unsplit. The quadratic JT effect lowers the $e$ sub-level of the $v^{\prime}{ }_{8}=1$ vibronic level and splits its $a_{1}+a_{2}$ sub-levels (Figure 3.7c). According to quantum chemical calculations and vibronic analysis (see below), the energy ordering of these three components (from low to high) is: $a_{1}, e, a_{2}$. Finally, when the SO interaction is taken into account (Figure 3.7d), both the $v^{\prime}=0$ level and the $e$ sub-level of the $v_{8}^{\prime}=1$ level are split into two $\mathrm{SO}$ components. Since the $\mathrm{SO}$ constant of $\mathrm{CaOCH}_{3}$ is positive, the $e_{3 / 2}$ component of the $v^{\prime}=0$ level is higher in energy than its $e_{1 / 2}$ component. For the $e$ sub-level of the $v^{\prime}{ }_{8}=1$ level, the energy ordering is the opposite. The SO interaction also couples the $a 1$ and $a 2$ sub-levels of the $v^{\prime} 8=1$ level so that the former (latter) sub-level shifts down (up). Both of these two sub-levels are $e_{1 / 2}$ levels $(|\Omega|=1=2)$.

The linear JT interaction couples the $v_{8}^{\prime}=1$ sub-levels to those of $v^{\prime}=0$ and, to a much lesser extent, energy levels of totally symmetric vibrational modes $\left(v_{1}-v_{4}\right)$, following the selection rule of $\Delta j=0$, where $j=l+\Lambda=2$ is the JT quantum number. ( $l$ is the vibrational 
angular momentum quantum number.) Due to the mixing of vibrational wave functions, transitions from the $v^{\prime \prime}=0$ level to the two $j=1 / 2$ sub-levels of the $v_{8}^{\prime}=1$ level gain intensities (solid arrows in Figure 7d). When the quadratic JT effect is involved, the vibronic coupling scheme becomes more complicated. In particular, interactions between $\Delta v=2$ levels are possible.33 The quadratic JT effect mixes vibronic wave functions with $\Delta j$ $= \pm 3$ so that transitions from the $v^{\prime \prime}=0$ level to the two $j=3 / 2$ sub-levels of the $v_{8}^{\prime}=1$ level are also allowed (dashed arrows in Figure 7d), although their intensities are small for the CaOCH3 according to our calculations.

To estimate the intensity of the nominally forbidden 810 LIF transition, the vibronic eigenfunctions are determined by a SOCJT2 calculation using the values of the JT parameters listed in chapter 4 . In order to be observed the $8_{0}^{1}$ band needs to borrow intensity from the allowed vibronic $\tilde{A}^{2} E-\tilde{X}^{2} A_{1}$ transitions from the zero-point level of the $\tilde{X}$ state. The only such allowed transitions within the $\tilde{A}$ state is to the vibrationless $\left(v^{\prime}=0\right)$ level and the totally symmetric vibrations. The latter has smaller oscillator strength than the former, so the most significant intensity "borrowing" is from the vibrationless level of the $\tilde{A}$ state. In addition, we consider the effect on the $8_{0}^{1}$ eigenfunctions from pJT mixing of the nearby $\tilde{B}^{2} A_{1}$ state, which experimentally has $\mathrm{T}_{00}=1757 \mathrm{~cm}^{-1}$ higher than that of the $\tilde{A}$ state. The coupling mechanism of the pJT effect is similar to that of the linear JT effect. The $v_{8}^{\prime}=1 \leftarrow$ $v^{\prime \prime}=0$ transition of the $\tilde{A}^{2} E-\tilde{X}^{2} A_{1}$ system also gains intensity via such a pJT interaction. The pJT derivative coupling term again can be calculated using CFour. Using these values, perturbation theory indicates that only the vibrationless level of the $\tilde{B}$ state provides a nonnegligible admixture of intensity into the $8_{0}^{1}$ transition but somewhat surprisingly the predicted intensity via pJT borrowing is actually greater than that via JT borrowing. 
Calculated intensities for the observed transitions are listed in Table 3 along with the experimental intensities. Since the calculated JT splittings of each spin-component are not resolved (see Figure 7 experimentally the calculated intensities of the $8_{0}^{1}$ band are obtained by summing the 4 nearly degenerate JT levels within each of the two spin-vibronic components. From Table 6 (“calc. 3", see also chapter 4) it is clear that while the vibronic coupling does provide intensity for $8_{0}^{1}$, there is still a quite significant discrepancy between the calculated and observed intensities. The calculated values of both spin components of the $8_{0}^{1}$ transition is about an order of magnitude too low compared to experiment, and while the higher-frequency spin component is predicted to be (slightly) weaker than the lower one, the calculated intensity ratio $(\sim 1: 1)$ is less than the experimental one $(4.5: 1)$. This problem could be solved by scaling the JT mixing coefficients to values which best reproduce the observed intensities. However, we find such a scaling requires values over an order of magnitude larger than the ones calculated by CFour. 


\subsubsection{Spin-Orbit coupling}

Faced with these results, we considered other possible intensity borrowing mechanisms. In particular, we considered SO coupling since it is a moderately strong interaction in $\mathrm{CaOCH}_{3}$. As with pJT coupling, the SO interaction can couple the relatively closely lying $\tilde{A}^{2} E$ and $\tilde{B}^{2} A_{1}$ states. For symmetry reasons, only the perpendicular components of the SO interaction couple these two states. In principle, the SO coupling interaction can be treated by the same Van-Vleck transformation as used for the pJT coupling in the chapter 4 . However, it has not been included previously in such treatments because the SO coupling only adds a constant term of the form, $\mathrm{S}^{2}-S^{2}$, to the effective $\tilde{A}$ state Hamiltonian, which does not shift the spectroscopically observed frequencies of a doublet state. Moreover, since the pJT and SO interactions do not couple a given $\tilde{A}$ state level to the same $\tilde{B}$ level, treating the two interactions together produces no additional terms from the Van Vleck transformation.

Therefore we treat the SO interaction separately since arguably that produces a clearer physical picture of this previously neglected interaction. We write the perpendicular terms of the SO Hamiltonian that couple the $\tilde{A}$ and $\tilde{B}$ states as:

$$
H_{S O}^{P}=a_{\perp}\left(L_{x} S_{x}+L_{y} S_{y}\right)
$$

$H_{S O}^{P}$ couples the $\tilde{A}$ and $\tilde{B}$ levels with the same vibrational quantum number $v$ and for which $\Lambda$ and $\Sigma$ are aligned anti-parallel. Note that there is no coupling between the $\tilde{B}$ state and the $\tilde{A}$ state when $\Lambda$ and $\Sigma$ are parallel. We deduce, by first-order perturbation theory, that for the anti-parallel spin and orbital components of a given vibrational level of the perturbed $\tilde{A}$ state, the new $\tilde{A}$ state basis functions can be written as 


$$
|\Lambda, l, j, \Sigma, \Omega\rangle^{\prime}=\left| \pm 1, l, l \pm \frac{1}{2}, \mp \frac{1}{2},-l \pm \frac{1}{2}\right\rangle+C_{A B}\left|0, l, l, \mp \frac{1}{2},-l \mp \frac{1}{2}\right\rangle
$$

where $\Omega=\Lambda+\Sigma-l, 33$ and

$$
C_{A B}=\frac{a_{\perp}}{\sqrt{2} \Delta E_{B-A}} \approx \frac{67}{\sqrt{2} \times 1757}=2.65 \times 10^{-2}
$$

where $\Delta E_{B-A}$ is the energy separation between the two electronic states. In Eq. (3), it is assumed

that the unpaired electron is entirely localized in an unperturbed $4 p$ orbital on $\mathrm{Ca}$, which yields

$a_{\perp}=a_{\|}$. The experimental values for $a_{\|}$and the energy separation, $\Delta E_{B-A}$, are used in Eq. (3). A derivation of Eq. (2) and Eq. (3) is presented in Section 5.5 of chapter 5. The $\tilde{A}$ state $v_{8}=1$ anti-parallel components can couple with those of $v=0$ of the $\tilde{A}$ state via the liner JT interaction, $H_{L J T}^{1}$. The effective coupling matrix element within the $\tilde{A}$ state becomes

$$
\left\langle\tilde{A}, v_{8}=1\left|H_{L J T}\right| \tilde{A}, v=0\right\rangle=k_{8}+2 C_{A B} \lambda_{A B}=0.85+2 \times 0.0265 \times 150 \mathrm{~cm}^{-1} \approx 8.8 \mathrm{~cm}^{-1}
$$

Where $k_{8}=0.85 \mathrm{~cm}^{-1}$ is the LJT coefficient for $\tilde{A}-$ state $v_{8}$, and

$$
\lambda_{A B}=\left\langle\tilde{B}, v_{8}=1\left|H_{L J T}\right| \tilde{A}, v=0\right\rangle=\left\langle\tilde{A}, v_{8}=1\left|H_{L J T}\right| \tilde{B}, v=0\right\rangle=150 \mathrm{~cm}^{-1}
$$


is calculated for the (linear) pJT term between the $\tilde{A}$ and $\tilde{B}$ state. The factor of 2 in front of $\lambda_{A B}$ on the right-hand-side of Eq. (4) reflects the fact that both the $v=0$ and the $v=1$ levels of the $\tilde{A}$ state is coupled to the $\tilde{B}$-state vibrational levels with the same $v$ (see Eq. (3) and Eq. (5)). The observed and calculated intensities of the individual spin components are given in Table 6 (“calc. 4" and "calc. 5"). In calc. 4, only diagonal SO correction is included, while in calc. 5, both diagonal and off-diagonal SO corrections are included. Clearly, both the overall intensity and relative spin-component intensities (calculated, 3.7, and experimental, 5.3) of the 810 spin components show reasonably good agreement between calc. 5 and observed values, likely confirming the SO mixing as the most important mechanism producing intensity to the $8_{0}^{1}$ transition. The overall FC factors and VBRs of other vibronic transitions calculated in this manner with JT coupling, pJT coupling, and complete SO corrections included are listed in Table 3 and Table 4 in comparison with experimental values. 


\subsubsection{The $8_{2}^{0}$ band in DF spectra}

The other observation involving $v_{8}$ is the appearance (when pumping the $\tilde{A}^{2} E_{3 / 2} 0_{0}^{0}$, but not when pumping $\tilde{A}^{2} E_{1 / 2} 0_{0}^{0}$ ) in the DF spectrum of a band whose frequency is consistent with emission to $2 v_{8}$ in the $\tilde{X}$ state. The possibility of this band being assigned to an impurity, as suggested earlier for the $\mathrm{X}$ and $\mathrm{Y}$ bands, is largely ruled out because the emission transition to the $8_{2}$ level was observed when LIF transitions to the $v_{4}^{\prime}$ $=1$ and $v_{3}^{\prime}=1$ levels were pumped (see Figure 3.5c and Figure 3.5e).

However, the FC factor for the $8_{2}^{0}$ transition, under the harmonic-oscillator approximation and BOA, is calculated to be essentially zero $\left(1 \times 10^{-4}\right)$, and would be exactly zero if the $\tilde{A}$ and $\tilde{X}$ states have identical geometries. However, this calculated value is about two orders of magnitude smaller than the experimentally observed value of $8 \times 10^{-3}$. In addition, it would predict comparably intense emissions when pumping to either the $\tilde{A}^{2} E_{1 / 2}$ or $\tilde{A}^{2} E_{3 / 2}$ component, something not observed experimentally. We can include the corrections for SO coupling and vibronic interaction in predicting the transition intensities in the DF spectra. Using the same Hamiltonian and parameters as we did for the $8_{1}^{0}$ calculations, we obtain the results given in Tables 4.12 and 4.13 for the intensities of the $8_{2}^{0}$ transitions pumping the $\tilde{A}^{2} E_{1 / 2}$ and $\tilde{A}^{2} E_{3 / 2} 0_{0}^{0}$ bands, respectively. The corrections are not negligible as we now obtain calculated intensities almost an order of magnitude larger than those without SO and vibronic interactions. These corrections arise almost entirely from the quadratic JT and pJT terms in the Hamiltonian and show a significantly larger intensity ratio

$(\approx 2: 5)$ for pumping the $\tilde{A}^{2} E_{3 / 2}$ compared to pumping the $\tilde{A}^{2} E_{1 / 2} 0_{0}^{0}$ band. Nonetheless, the overall calculated intensity is still an order of magnitude smaller than the experimentally 
observed one. Therefore we doubt that either vibronic coupling or SO coupling is a sufficient explanation for the strength of the $8_{2}^{0}$ transition observed experimentally. Moreover, for any intensity borrowing mechanism within the $\tilde{A}$ state, it would be impossible for the VBRs of the $8_{1}^{0}$ vs $8_{2}^{0}$ and $8_{1}^{0}$ vs $8_{2}^{0}$ to switch between the LIF and DF spectra. (This statement does not hold if unique perturbations exist between the $v_{8}$ levels in the $\tilde{A}$ or $\tilde{X}$ state by a third state, e.g. the $\tilde{B}$ state. However, a mechanism for such perturbations of appropriate magnitude is not at all obvious.)

Given this situation, we believe that the most likely explanation for the DF band observed $278 \mathrm{~cm}^{-1}$ red of the origin when pumping $\tilde{A}^{2} E_{3 / 2} 0_{0}^{0}$, is a result of intensity borrowing by the $8_{2}^{0}$ Level from $v_{3}$ and $v_{4}$ in the $\tilde{X}$ state. There is clear evidence of coupling between these levels elsewhere in the emission spectrum (see Section 4.3). The $8_{2}^{0}$ emission occurs relatively strongly when pumping the $4_{0}^{1}$ and $3_{0}^{1}$ bands. Indeed a long progression including $8_{4}^{0}$ and $8_{6}^{0}$ appears when pumping the latter band. Presumably the mixing of the $8_{v^{\prime \prime}}^{0}\left(v^{\prime \prime}\right.$ is even) and the totally symmetric levels is caused by anharmonic coupling in the $\tilde{X}$ state. Therefore we have used the routines available in CFour to calculate cubic and quartic coupling coefficients between $8_{2}$ and both $3_{1}$ and $4_{1}$ in the $\tilde{X}$ state. These values are listed in Table 4.14 and 4.15 of chapter 4 and one can see that they are quite significant. Combining these values with the cubic and quartic normal coordinate operators and the use of perturbation theory allows one to estimate the admixture of $3_{1}$ and $4_{1}$ characters into the $a_{1}$ component of the nominal $8_{2}$ wavefunctions and estimate qualitatively via perturbation theory its intensity borrowing from these allowed bands. The resulting estimate for the intensity of the $8_{2}^{0}$ transition is on the order of $10 \%$ of the $4_{1}^{0}$ transition, corresponding to a 
net intensity of about $0.2 \%$ of the origin, consistent with the observed value of $<1 \%$ given in Table 3.4.

The final question involves the dependence of the $8_{2}^{0}$ emission upon which SO component of the origin band is pumped. Why is the $8_{2}^{0}$ emission observed only from the $\tilde{A}^{2} E_{3 / 2}$ level but not the $\tilde{A}^{2} E_{1 / 2}$ level? While it is not presently possible to calculate anharmonic terms for the degenerate $\tilde{A}$ state with the CFour software, we believe that such terms are likely responsible for the spin-dependence. Note that Figure 5e shows a relatively strong spin dependence of the $3^{1} 8^{2}$ emission intensity on the pumped SO components of the $3_{1}^{0}$ band. A stronger $3^{1} 8^{2}$ emission is observed when pumping to the $\tilde{A}^{2} E_{3 / 2} 3^{1}$ level than the $\tilde{A}^{2} E_{1 / 2} 3^{1}$ level. Such dependence could be transferred to the origin band by the anharmonic terms in the $\tilde{A}$ state. Further investigation of the $v_{8}$ levels of the $\tilde{X}$ state as well as the $\tilde{A}$ state by rotationally resolved spectroscopy would be useful for sorting out the details of the intensity borrowing mechanism ${ }^{90}$. 


\subsection{Comparison with previous experimental values of VBRs}

Recently, Kozyryev et al.13 reported VBRs of the $\tilde{A}^{2} E \rightarrow \tilde{X}^{2} A_{1}$ and $\tilde{B}^{2} A_{1} \rightarrow$ $\tilde{X}^{2} A_{1}$ transitions of $\mathrm{CaOCH}_{3}$ measured by DF using a continuous-wave (cw) excitation laser, under jet-cooled conditions to excite individual rotational level. In Table 3.5, VBRs determined in the present work are compared with those reported by Kozyryev et al. (Note that authors of Ref. ${ }^{71}$ did not adopt the Herzberg notation in numbering the vibrational modes.) Inspecting Table 5, we see that there is generally good consistency between the present results and those of Ref. ${ }^{71}$. However, it must be noted that our measurements have a significantly larger uncertainty. Nonetheless, they represent confirmation of most of the previous results using a largely independent approach, e.g. pulsed vs cw laser, use of CRD spectroscopy to determine the $0_{0}^{0}$ band intensity, etc.

There are, however, two matters concerning the $0_{0}^{0}$ band that deserves further comment. While the $0_{0}^{0}(e 1=2)$ VBRs agree with experimental error, the values of Ref. ${ }^{71}$ do lie towards the upper end of our expected error. We mentioned earlier that there were two possible systematic errors, both of which could increase the observed emission from $0_{0}^{0}$ and hence it's VBR. (See Section 2.) If one considers upper limits estimated for either effect we find that the origin VBR increases to 0.928 , which is near that of Ref. ${ }^{71}$.

There is also a relatively large discrepancy between the VBRs for the $3_{1}^{0}$ bands

determined in our work and reported in Ref. ${ }^{71}$. The VBR for the $\tilde{A}^{2} E_{3 / 2} \rightarrow \tilde{X}^{2} A_{1} 3_{1}^{0}$ band is reported as 0.006 in Ref. 13, while the $\tilde{A}^{2} E_{1 / 2} \rightarrow \tilde{X}^{2} A_{1} 3_{1}^{0}$ band was not observed. (The lower VBR value is also much more consistent with that predicted by our quantum chemical calculations.) In the present work, these VBRs from experiment are 0.025 and 0.028 , respectively. The reasons for this discrepancy are unidentified at this stage but might be 
attributed to difference in experimental apparatuses or conditions. A cw laser was used in Ref. ${ }^{71}$ to excite individual rotational lines in the electronic transitions, whereas a pulsed laser was used in our work, which might affect the measurements of intensities of weak transitions. Alternatively, an "impurity" band like $\mathrm{X}$ or $\mathrm{Y}$ in Figure 5 might overlap the position of the $3_{1}^{0}$ band in our DF spectra. However, we have tried without success varying conditions to alter the intensity of these $3_{1}^{0}$ bands compared to other emission bands. Nonetheless, we have considered the hypothetical situation where the $3_{1}^{0}$ relative intensity is reduced to that reported in Ref. 13 . We find a value of the $0_{0}^{0}$ VBR increased to 0.915 , while the calculation also demonstrates that the VBRs of the other bands are little changed. The other point concerns the VBR of the $0_{0}^{0}\left(e_{3 / 2}\right)$ band which Ref. 13 finds to be larger than $0_{0}^{0}$ $\left(e_{1 / 2}\right)$, with the difference beyond their uncertainty. (Our results with larger uncertainties cannot distinguish between the $e_{1 / 2}$ and $e_{3 / 2}$ values.) We find that surprising for the following reason. Our excitation spectrum shows that $8_{1}^{0}$ is clearly observable. Moreover, our vibronic analysis shows that, mainly due to energy approximate, $8_{1}^{0}\left(e_{3 / 2}\right)$ steals more intensity from the origin than does $8_{1}^{0}\left(e_{1 / 2}\right)$ and indeed does a reasonable job of predicting its magnitude. Clearly, this stolen intensity should result in diminished emission on the two $0_{0}^{0}$ band spin-orbit components with the decrease for the $e_{3 / 2}$ component significantly greater. VBRs for the origin transitions, $\tilde{A}^{2} E_{1 / 2} \rightarrow \tilde{X}^{2} A_{1}$ and $\tilde{A}^{2} E_{3 / 2} \rightarrow \tilde{X}^{2} A_{1}$ are not always consistent with Ref. ${ }^{71}$ within the error bars. This may be explained by the fact that several weak emission transitions were observed in our DF spectra but are absent in the DF spectra reported in Ref. ${ }^{71}$. 


\subsection{Implications to direct laser cooling of $\mathrm{CaOCH}_{3}$ and TRSV}

\section{measurements}

We now discuss the implication of the determined FC factors to direct laser cooling of the $\mathrm{CaOCH}_{3}$ radical. In such an experiment, pre-cooled molecules are excited to the $\tilde{A}^{2} E_{1 / 2}$ state. Besides the origin transitions, spontaneous emission to both the $4_{1}$ level and even the $3_{1}$ level is significant. If a cooling laser is used to excite the origin band and two re-pumping lasers are employed to return the population from the $4_{1}$ and $3_{1}$ levels to the cooling cycle, the sum of VBRs for spontaneous emission transitions to $\tilde{X}^{2} A_{1}$-state vibrational levels other than $0_{0}, 3_{1}$ and $4_{1}$ is 0.012 , which implies that a molecule will experience on average $\sim 84$ scattering events before it decays to vibrational "dark" states that are not addressed by the re-pumping lasers.

Measurement of energy level structure of fundamental and overtone levels of the Ca-O-C bending mode $\left(v_{8}\right)$ and transitions involving them is a prerequisite of the proposed investigation of TRSV interactions and the detection of eEDM.18 The $v^{\prime \prime}{ }_{8}=1$ state is the proposed eEDM measurement state. Population transfer from the ground vibrational

level of the $\tilde{X}^{2} A_{1}$ state to the $v^{\prime \prime}{ }_{8}=1$ state can be achieved using stimulated Raman adiabatic passage (STIRAP)39-42 using $v_{8}^{\prime}=1$ as the intermediate state. As demonstrated in the present work, JT, pJT, and SO interactions significantly increase the efficiency of STIRAP process by breaking the BOA and harmonic oscillator approximations and mixing the $v_{8}$ states with non-degenerate totally symmetric vibrational states $\left(v_{3}\right.$ and $\left.v_{4}\right)$. 


\subsection{Conclusions}

We report vibrationally resolved LIF and DF spectra of the $\tilde{A}^{2} E \rightarrow \tilde{X}^{2} A_{1}$ transition of the calcium methoxide radical. The vibrational assignment has been made on the basis of CASSCF- and CC-calculated vibrational frequencies and FC factors for the allowed vibronic transitions. It has been found that both ab initio methods predict the vibrational frequencies to a significant degree of accuracy. These calculations also do a reasonably good job for the intensity of the allowed, strong transitions. However other forbidden transitions are weakly observed in the spectrum which is not predicted to have intensity by either calculation. These vibronic transitions, $8_{1}^{0}$ in the LIF spectra and $8_{2}^{0}$ in the DF spectra, are not predicted under the BOA and harmonic oscillator approximations. The former, observed in the excitation spectrum, are ascribed to the pJT and SO interaction between the $\tilde{A}^{2} E$ and the $\widetilde{B}^{2} A_{1}$ states. Correspondingly, the latter observed in DF spectra is due to emission from the origin band to levels involving $8_{v^{\prime \prime}}\left(v^{\prime \prime}=\right.$ even) are attributed primarily to the anharmonic coupling of these levels with $3_{1}$ and $4_{1}$ in the $\tilde{X}$ state. Such nominally "forbidden” but observed transitions are important for two reasons. They alter the efficiency of laser cooling, and they offer an opportunity to access levels of particular significance to the detection of TRSV interactions. In the present work, relative intensities were determined in LIF and DF measurements, but our measurements have two limitations. First, the fluorescence signal is contaminated by the excitation laser scattering. Second, the excitation transitions of the origin band are power saturated. The second issue could be largely avoided by using continuous-wave (cw) excitation laser sources. 
However, weak transitions could not be observed in the LIF/DF experiment using cw excitation sources. In the present work, we compensate for these two issues by making use of the jet-cooled pulsed-laser CRD spectra. The signal-to-noise ratio of the current CRD measurement is somewhat lower than the LIF experiment. It can be significantly improved if cw laser sources are used. To achieve higher accuracy in determining the intensities, a two-beam CRD spectroscopy technique may be applied ${ }^{91}$. The frequency of the first laser is fixed and in resonance with a certain transition, e.g., the $\tilde{A}^{2} E \rightarrow \tilde{X}^{2} A_{1}$ origin transition, whereas that of the second laser is tuned. The transition intensities, measured using the second (scanning) laser, are normalized by that of the first (fixed frequency) laser. Uncertainty in transition intensity measurement induced by the fluctuation of ablation laser power, free radical production, and other experimental factors can, therefore, be largely eliminated. 
Table 3.1: Geometric parameters, rotational constants, and $\tilde{A}-\tilde{X}$ excitation energy of the $\mathrm{CaOCH}_{3}$ radicals

\begin{tabular}{|c|l|l|l|l|l|l|}
\hline & \multicolumn{2}{|c|}{${\text { Calc. } 1^{\mathrm{a}}}$} & \multicolumn{2}{c|}{ Calc. $2^{\mathrm{b}}$} & \multicolumn{2}{c|}{ expt. $^{\mathrm{c}}$} \\
\cline { 2 - 7 } & $\tilde{X}^{2} A_{1}$ & $\tilde{A}^{2} E^{d}$ & $\tilde{X}^{2} A_{1}$ & $\tilde{A}^{2} E^{d}$ & $\tilde{X}^{2} A_{1}$ & $\tilde{A}^{2} E^{d}$ \\
\hline$r_{\mathrm{CaO}}(\AA)$ & 2.012 & 1.982 & 2.139 & 2.113 & 1.962 & 1.942 \\
$r_{\mathrm{CO}}(\AA)$ & 1.372 & 1.373 & 1.414 & 1.420 & 1.411 & 1.411 \\
$r_{\mathrm{CH}}(\AA)$ & 1.090 & 1.090 & 1.095 & 1.094 & 1.094 & 1.092 \\
$\angle \mathrm{OCH}($ deg. $)$ & 111.9 & 111.7 & 111.5 & 111.1 & 111.3 & 111.0 \\
\hline$A(\mathrm{~cm}-1)$ & 5.447 & 5.440 & 5.366 & 5.355 & 5.375 & 5.367 \\
$B(\mathrm{~cm}-1)$ & 0.115 & 0.117 & 0.104 & 0.105 & 0.116 & 0.118 \\
\hline$\Delta E A-X(\mathrm{~cm}-1) e$ & 15359 & \multicolumn{5}{|c|}{15918} \\
\hline
\end{tabular}

${ }^{a}$ Calculated at the CAS(3,6)/cc-pVTZ level of theory.

${ }^{b}$ Calculated at the EOMEE-CCSD/cc-pVTZ level of theory.

${ }^{c}$ Ref. 14.

${ }^{a}$ Geometry optimization is at the conical intersection.

${ }^{e}$ Defined as energy separation between the ground state minimum and the conical intersection of the $\tilde{A}^{2} \mathrm{E}$ state. 
Table 3.2: Harmonic vibrational frequencies of CaOCH3 in the $\tilde{X}^{2} A_{1}$ and $\tilde{A}^{2} E$ states (in cm ${ }^{-}$ ${ }^{1}$ ). Vibrational modes are numbered following the Herzberg convention.

\begin{tabular}{|c|c|c|c|r|r|r|l|c|}
\hline mode & sym. & \multicolumn{4}{|c|}{$\tilde{X}^{2} A_{1}$ state freq } & \multicolumn{2}{|c|}{$\tilde{A}^{2} E$ state freq } & description \\
\cline { 3 - 9 } & & calc. $1^{a}$ & calc. $2 b$ & expt. & calc. $1^{\text {a }}$ & calc. $2^{\mathrm{b}}$ & expt. & \\
\hline $\mathrm{v}_{1}$ & $a_{1}$ & 3078 & 3001 & & 3095 & 3016 & & sym. CH stretch \\
\hline $\mathrm{v}_{2}$ & $a_{1}$ & 1622 & 1504 & 1450 & 1611 & 1502 & 1387 & $\mathrm{CH}_{3}$ umbrella \\
\hline $\mathrm{v}_{3}$ & $a_{1}$ & 1276 & 1185 & 1157 & 1280 & 1181 & 1156 & asym. CaOC stretch \\
\hline $\mathrm{v}_{4}$ & $a_{1}$ & 487 & 508 & 490 & 507 & 512 & 502 & $\mathrm{CaO}$ stretch \\
\hline $\mathrm{v}_{5}$ & $e$ & 3106 & 3052 & & 3127 & 3074 & & asym. CH stretch \\
\hline $\mathrm{v}_{6}$ & $e$ & 1616 & 1523 & & 1616 & 1523 & & $\mathrm{CH}_{3}$ scissoring \\
\hline $\mathrm{v}_{7}$ & $e$ & 1290 & 1190 & & 1291 & 1189 & & $\mathrm{CH}_{3}$ rock \\
\hline $\mathrm{v}_{8}$ & $e$ & 152 & 124 & & 157 & $122 c$ & 149 & $\mathrm{CaOC}_{3}$ bending \\
\hline
\end{tabular}

$a$ Calculated at the CAS(3,6)/cc-pVTZ level of theory.

$b$ Calculated at the EOMEE-CCSD/cc-pVTZ level of theory.

${ }^{C}$ Including JT and pJT effects shifts this band to a predicted value of $140 \mathrm{~cm}^{-1}$. 


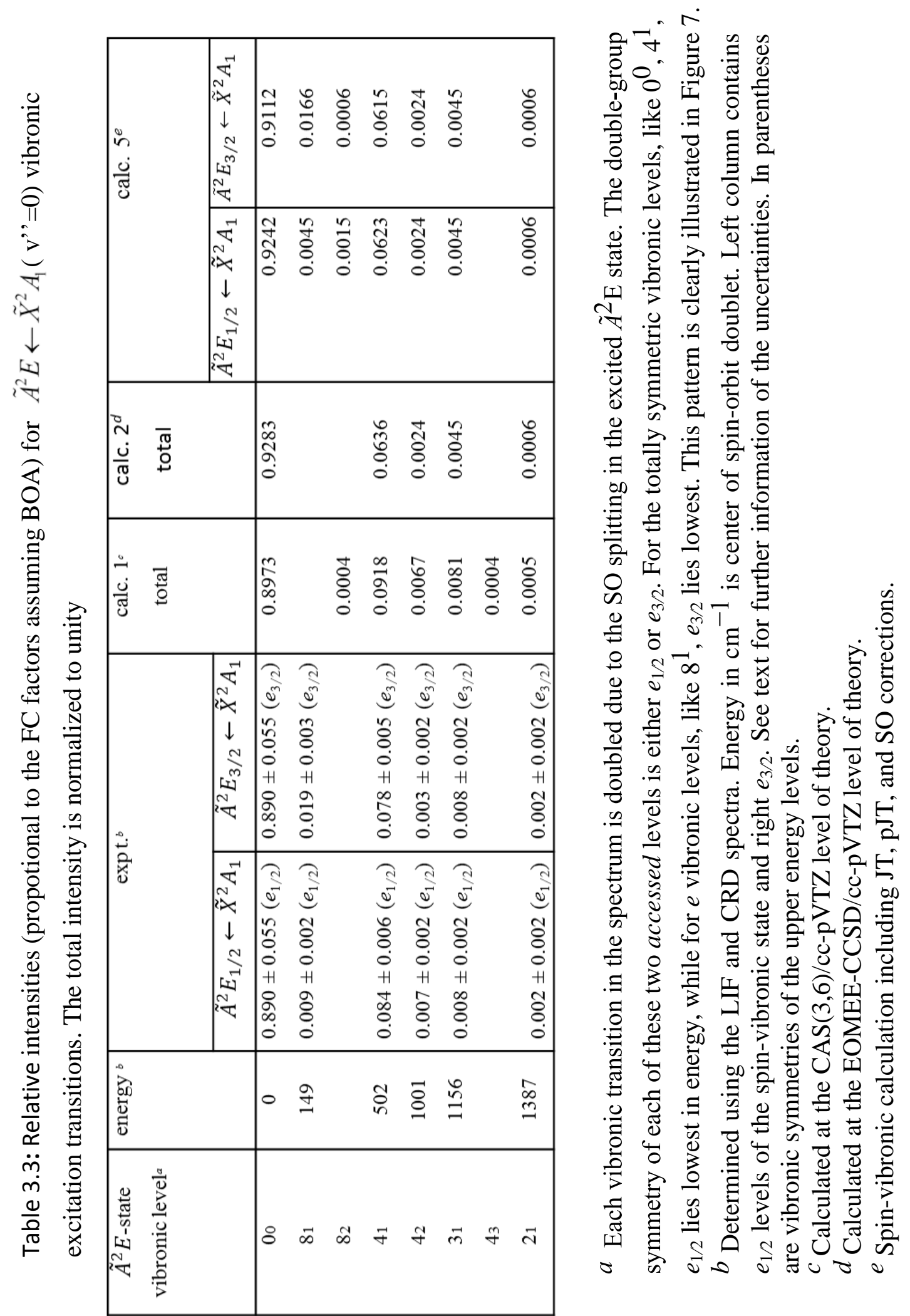




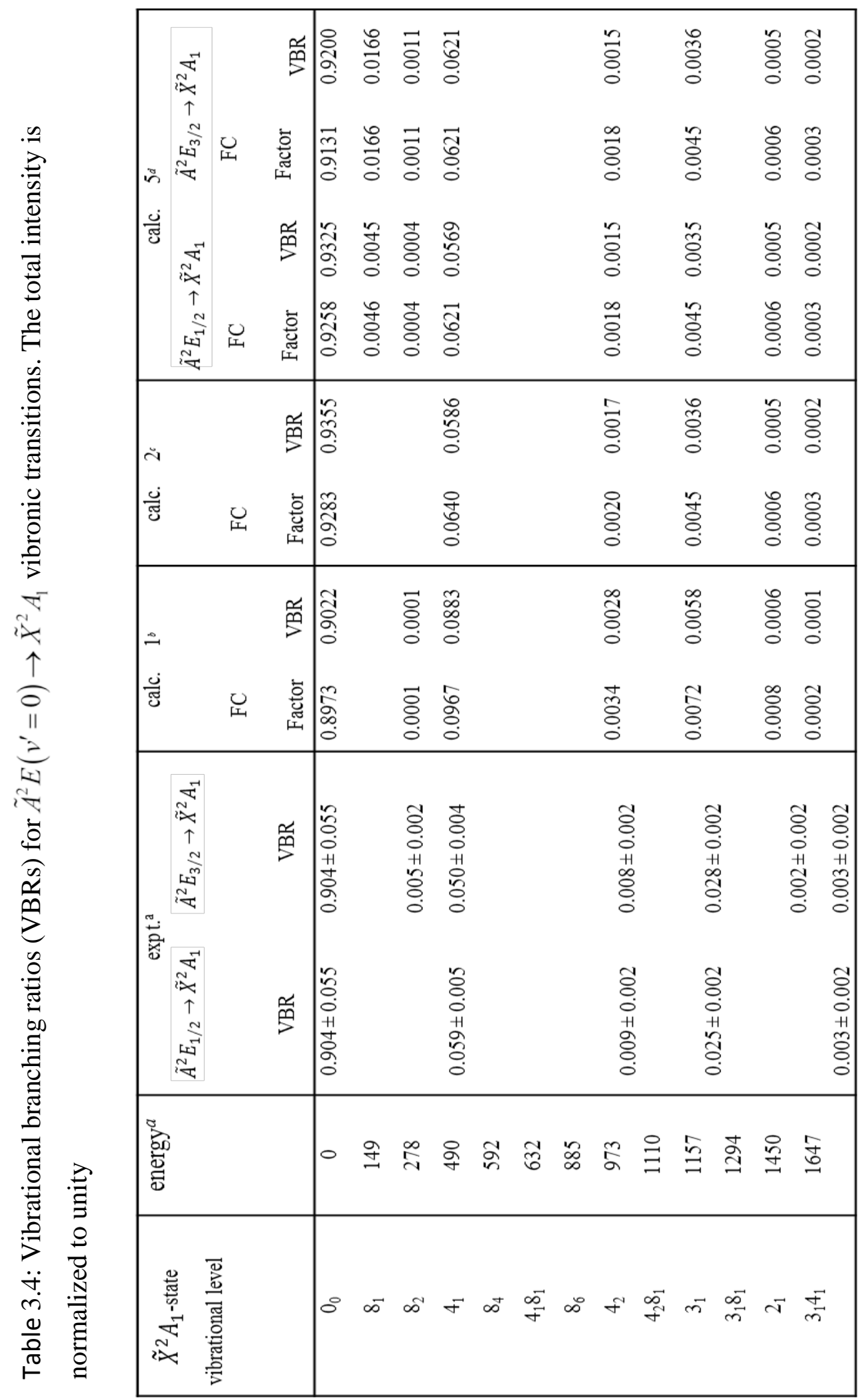


${ }^{a}$ Determined using the DF spectra. Left column refers to emission from the $e_{1 / 2}$ spin-vibronic level of the $A^{\tilde{}}$ state, left $e_{3 / 2}$. See text for further information about uncertainties.

${ }^{b}$ Calculated at the CAS(3,6)/cc-pVTZ level of theory.

${ }^{c}$ Calculated at the EOMEE-CCSD/cc-pVTZ level of theory.

${ }^{d}$ Spin-vibronic calculation including JT, pJT, and SO corrections.

Table 3.5: Comparison of VBRs for $\tilde{A}^{2} E \rightarrow \tilde{X}^{2} A_{1}$ emission transitions determined in the present work reported in Ref. 13.

\begin{tabular}{|c|cc|cc|}
\hline$\tilde{X}^{2} A_{1}$-state & \multicolumn{2}{|c|}{$\tilde{A}^{2} E_{1 / 2} \rightarrow \tilde{X}^{2} A_{1}$} & \multicolumn{2}{c|}{$\tilde{A}^{2} E_{3 / 2} \rightarrow \tilde{X}^{2} A_{1}$} \\
vibrational levels & this work & Ref. 13 & this work & Ref. 13 \\
\hline $0_{0}$ & $0.904 \pm 0.055$ & $0.931 \pm 0.003$ & $0.904 \pm 0.055$ & $0.945 \pm 0.004$ \\
$8_{2}$ & & & $0.005 \pm 0.002$ & \\
$4_{1}$ & $0.059 \pm 0.005$ & $0.063 \pm 0.003$ & $0.050 \pm 0.004$ & $0.055 \pm 0.004$ \\
$4_{2}$ & $0.009 \pm 0.002$ & & $0.008 \pm 0.002$ & \\
$3_{1}$ & $0.025 \pm 0.002$ & $0.006 \pm 0.003$ & $0.028 \pm 0.002$ & \\
$2_{1}$ & & & 0.002 & \\
$3_{1} 4_{1}$ & $0.003 \pm 0.002$ & & $0.003 \pm 0.002$ & \\
\hline
\end{tabular}


Table 3.6: Calculated relative intensities for the $\tilde{A}^{2} E \leftarrow \tilde{X}^{2} A_{1} 0_{0}^{0}$ and $8_{0}^{1}$ excitation transitions

\begin{tabular}{|c|c|c|c|c|c|}
\hline$\tilde{A}^{2} E$-state & calc. 2 & calc. 3 & calc. 4 & calc. 5e & expt. \\
vibronic level $^{\mathrm{a}}$ & & & & & \\
\hline $00\left(\mathrm{e}_{1 / 2}\right)$ & & 0.9356 & 0.9336 & 0.9324 & 0.890 \\
$00\left(\mathrm{e}_{3 / 2}\right)$ & 0.9283 & 0.9178 & 0.9336 & 0.9194 & 0.890 \\
$81\left(\mathrm{e}_{3 / 2}\right)$ & 0 & 0.0019 & 0.0030 & 0.0168 & 0.019 \\
$81\left(\mathrm{e}_{1 / 2}\right)$ & & 0.0019 & 0.0028 & 0.0046 & 0.009 \\
\hline
\end{tabular}

${ }^{a}$ Each vibronic transition in the spectrum is doubled due to the SO splitting in the excited $\tilde{A}^{2} \mathrm{E}$

state. The double-group symmetry of each of these two accessed levels is either $e_{1 / 2}$ or $e_{3 / 2}$. For the totally symmetric vibronic levels, like $0^{0}, 4^{1}, e_{1 / 2}$ lies lowest in energy, while for $e$ vibronic levels, like $8^{1}, e_{3 / 2}$ lies lowest. This pattern is clearly illustrated in Figure 7. In parentheses are vibronic symmetries of the upper energy levels.

${ }^{b}$ Calculated at the EOMEE-CCSD/cc-pVTZ level of theory.

${ }^{c}$ Spin-vibronic calculations including JT and diagonal SO coupling.

${ }^{d}$ Spin-vibronic calculations including JT, pJT coupling and diagonal SO coupling.

${ }^{e}$ Spin-vibronic calculations including JT, pJT coupling and complete SO corrections. 


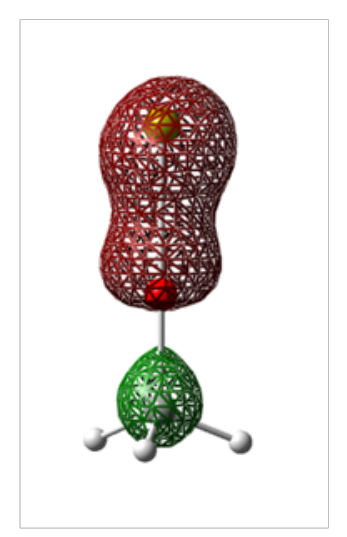

$\mathrm{Ca} 3 s+\mathrm{O} 2 s$

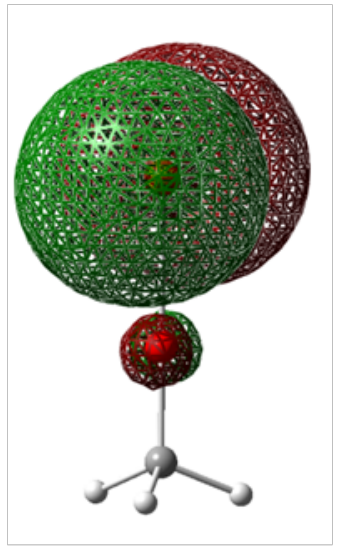

Ca $4 p_{\mathrm{x}}$

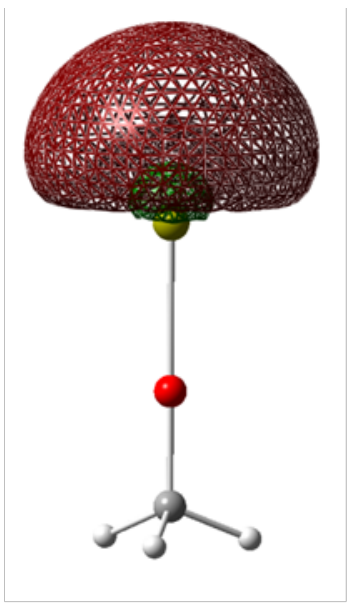

Ca $4 s$

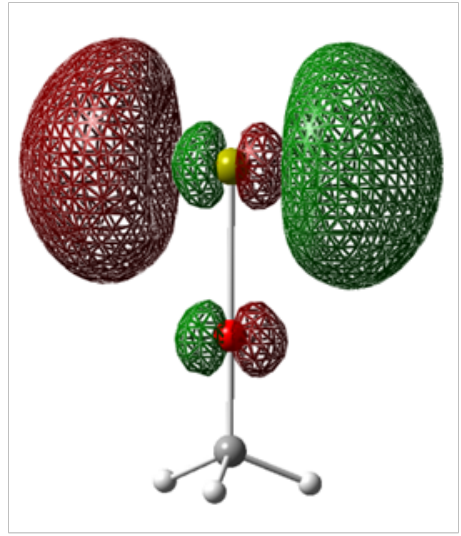

$\mathrm{Ca} 4 p_{\mathrm{y}}$

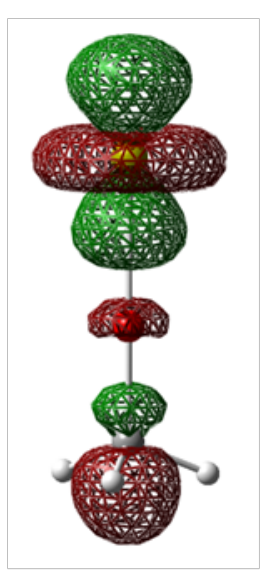

$\mathrm{Ca} 3 d_{z}^{2}$

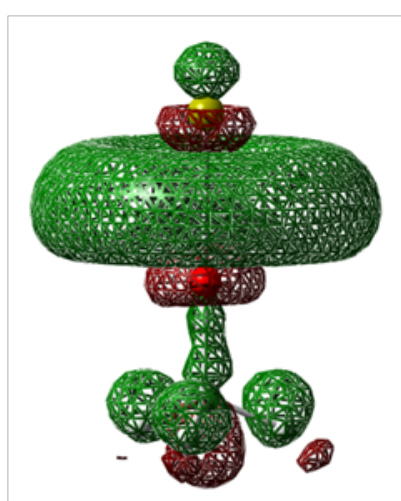

Ca $4 p_{z}$

Figure 3.1: The molecular orbitals of $\mathrm{CaOCH}_{3}$ included in CASSCF calculation 
(c)
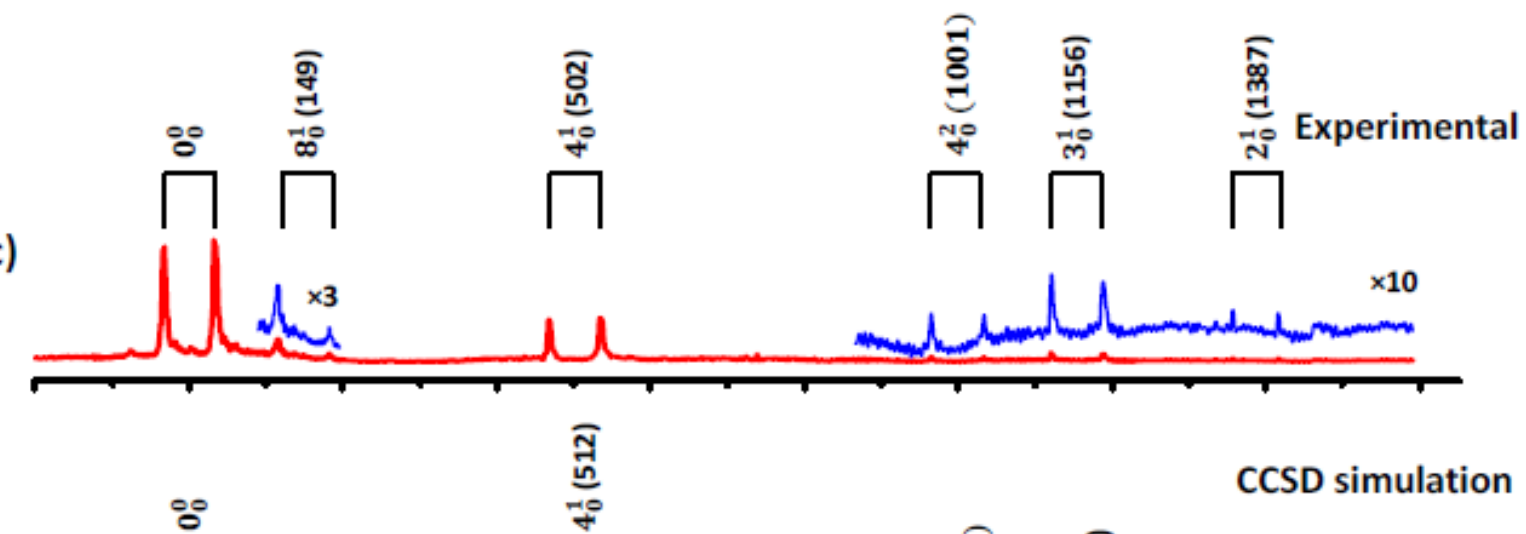

(b)
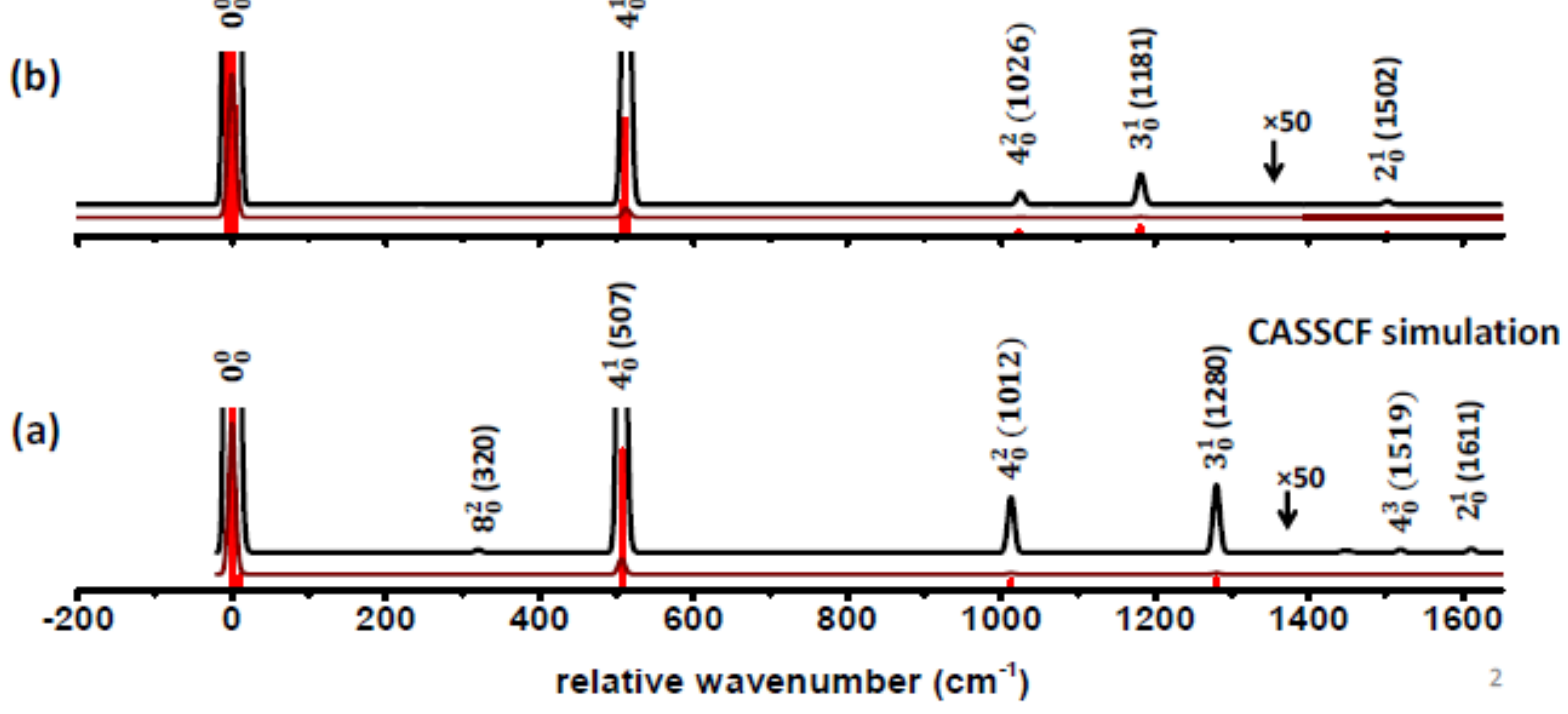

Figure 3.2: (a and b) simulated and (c) experimental LIF spectra of the $\tilde{A}^{2} E \leftarrow \tilde{X}^{2} A_{1}$ transition of $\mathrm{CaOCH}_{3}$. Wavenumbers are relative to the origin band at $15925 \mathrm{~cm}^{-1}$. Numbers in parentheses are relative wavenumbers in $\mathrm{cm}^{-1}$. The peaks in each doublet correspond to transitions to the $\tilde{A}^{2} E_{1 / 2}$ (lower frequency) and $\tilde{A}^{2} E_{3 / 2}$ (higher frequency) SO components. 


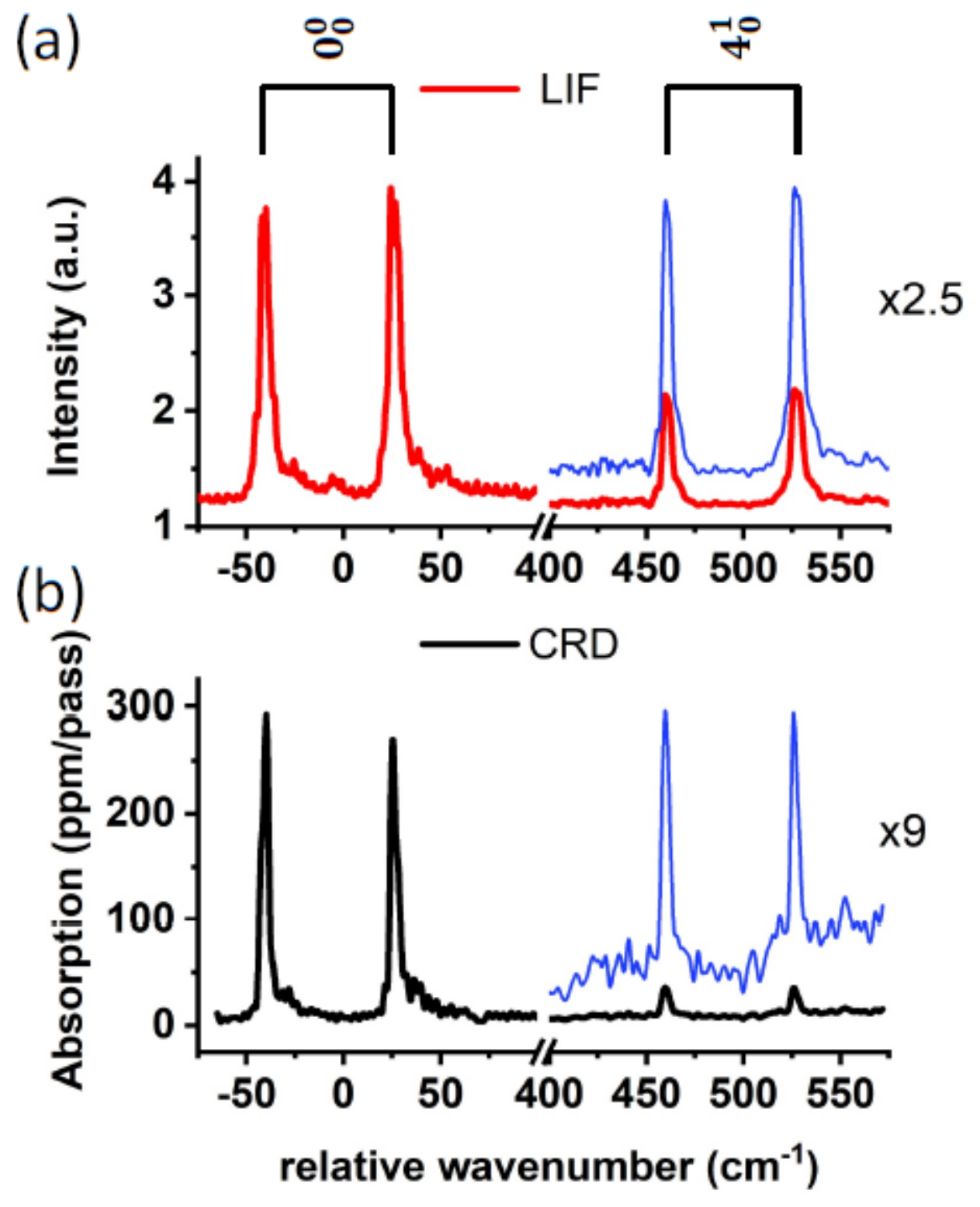

Figure 3.3: Comparison of (a) LIF and (b) CRD spectra of the origin and $4^{1}$ band transitions of $\mathrm{CaOCH}_{3}$ that shows the power saturation of the origin transitions in the LIF experiment 


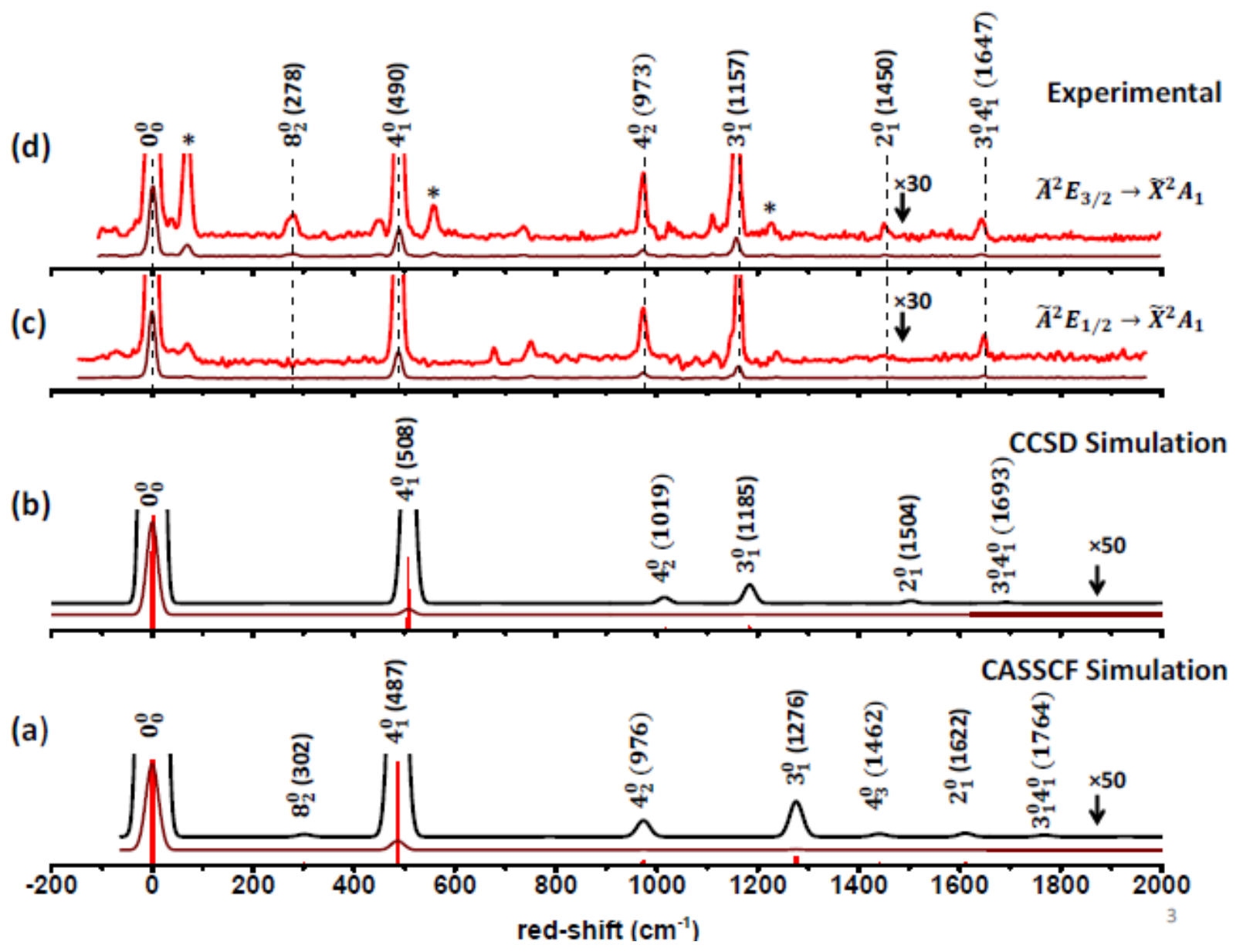

Figure 3.4: (a and b) Simulated DF spectrum of the $\tilde{A}^{2} E \leftarrow \tilde{X}^{2} A_{1}$ origin transition of $\mathrm{CaOCH}_{3}$. DF spectra obtained by pumping the (c) $\tilde{A}^{2} E_{1 / 2} \leftarrow \tilde{X}^{2} A_{1}$ and (d) $\tilde{A}^{2} E_{3 / 2} \leftarrow$ $\tilde{X}^{2} A_{1}$ origin transitions. Numbers in parentheses are red-shift in $\mathrm{cm}^{-1}$. The asterisked peaks in (d) are due to collision-induced $\tilde{A}^{2} E_{3 / 2} \leftarrow \tilde{X}^{2} A_{1}$ population transfer following laser excitation. (See text). 


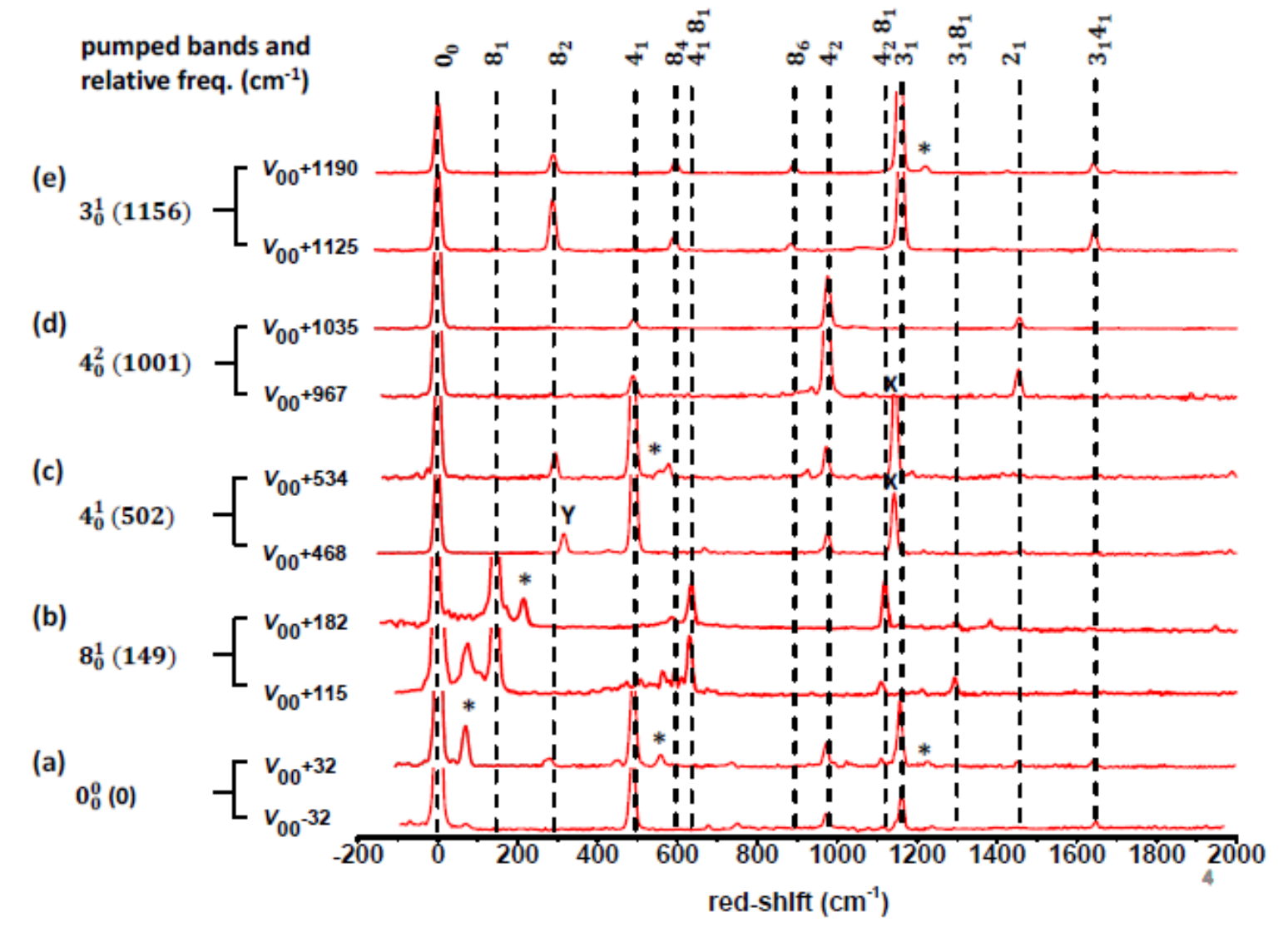

Figure 3.5: DF spectra obtained by pumping different vibronic bands of the $\tilde{A}^{2} E \leftarrow \tilde{X}^{2} A_{1}$ transition. The asterisked peaks are due to collision-induced $\tilde{A}^{2} E_{3 / 2} \leftarrow \tilde{A}^{2} E_{1 / 2}$ population transfer following laser excitation. " $\mathrm{X}$ " and "Y" denote unidentified transitions. (See text) 


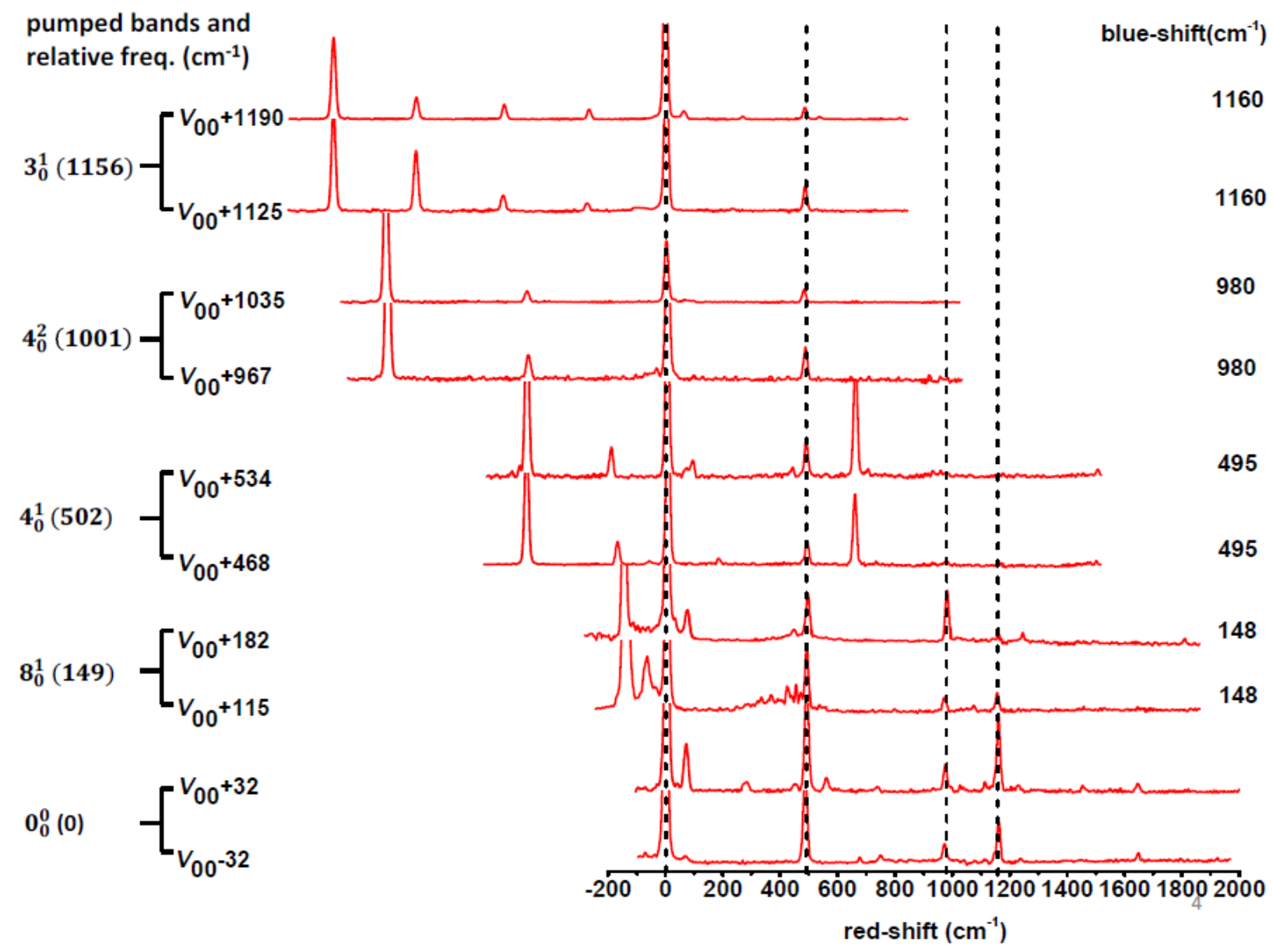

Figure 3.6: DF spectra obtained by pumping different vibronic bands of the $\tilde{A}^{2} E \leftarrow$ $\tilde{X}^{2} A_{1}$ transition. Spectra of bands other than origin band in the LIF spectrum are blueshifted. " $X$ " and "Y" denote unidentified transitions. (See text) 


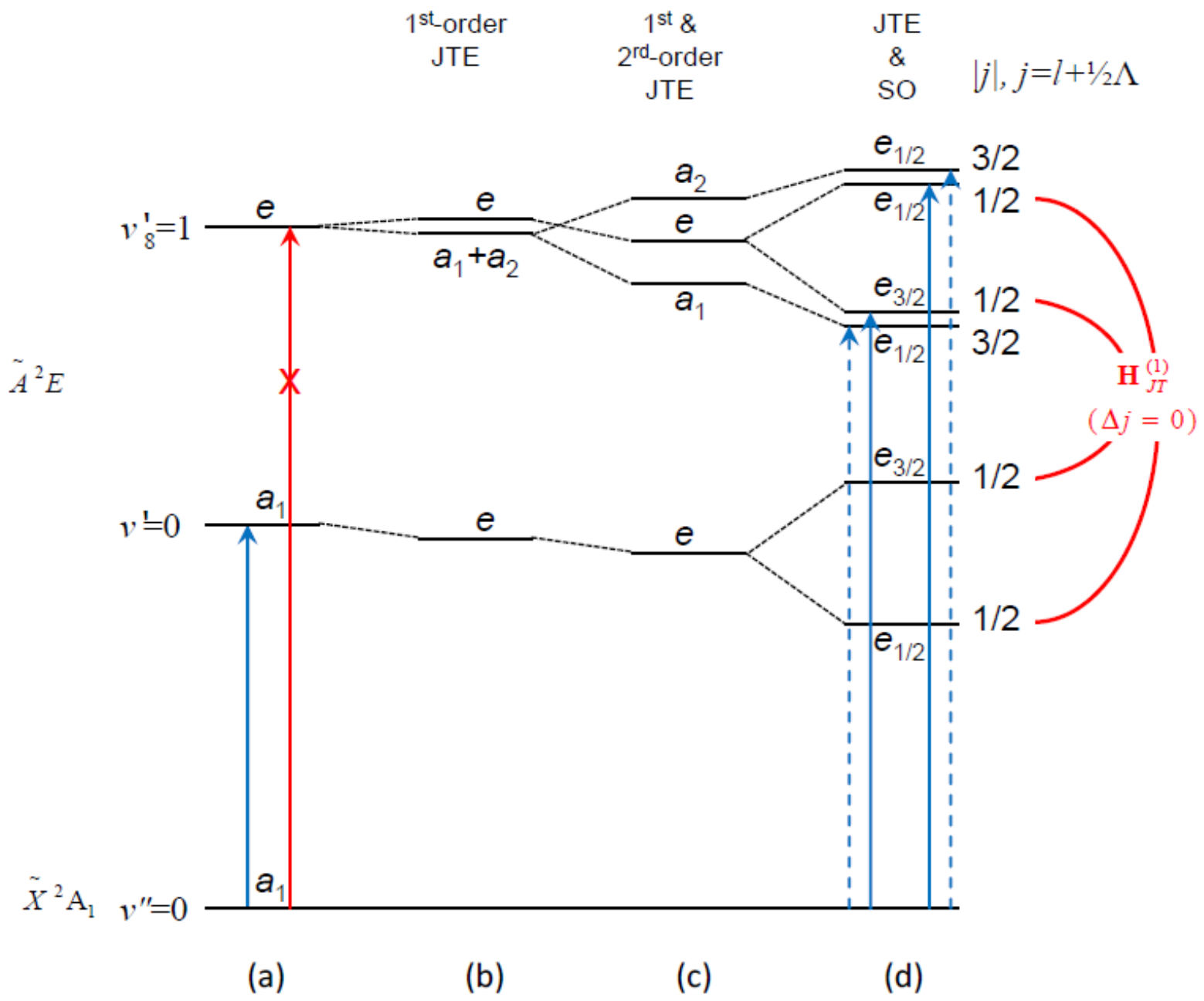

Figure 3.7: Qualitative energy level scheme of the spin-vibronic energy levels of the $\tilde{A}^{2} E$ state of $\mathrm{CaOCH}_{3}$ and $\tilde{A}^{2} E \leftarrow \tilde{X}^{2} A_{1}$ vibronic transitions. (a) in the absence of JT and SO interactions. (b) With the linear JT effect included. (c) With both linear and quadratic JT effect included. (d) With both JT and SO interaction included 


\section{CHAPTER IV}

\section{THEORY OF PSEUDO-JAHN-TELLER MOLECULES}

\subsection{Estimated relative errors of experimentally determined Franck- \\ Condon (FC) factors}

The Franck-Condon (FC) factor of the $i^{\text {th }}$ vibronic band in an electronic transition system with $N$ vibronic bands can be calculated as:

$$
F C_{i}=\frac{I_{i}}{\sum_{n=1}^{N} I_{n}}
$$

where $I_{n}$ 's are the integrated line intensities of the vibronic bands in the excitation spectrum so that $I_{n}$ is proportional to $F C_{n}$ and independent of the transition frequency. The error of $F C_{i}$, denoted by $\sigma_{F C i}$, can be calculated from experimental errors of the line intensities, $\sigma_{I n}$ 's, using the standard error propagation relation:

$$
\sigma_{F C_{i}}^{2}=\sum_{n=1}^{N} \sigma_{I_{n}}^{2}\left(\frac{\partial F C_{i}}{\partial I_{n}}\right)^{2}=\sigma_{I_{i}}^{2}\left(\frac{\partial F C_{i}}{\partial I_{i}}\right)^{2}+\sum_{n \neq i} \sigma_{I_{n}}^{2}\left(\frac{\partial F C_{n}}{\partial I_{n}}\right)^{2}
$$


The two derivatives on the right side of Eq. (4.1.2) can be easily evaluated:

$$
\frac{\partial F C_{i}}{\partial I_{i}}=\frac{\sum_{n=1}^{N} I_{n}-I_{i}}{\left(\sum_{n=1}^{N} I_{n}\right)^{2}}=\frac{\sum_{n \neq i} I_{n}}{\left(\sum_{n=1}^{N} I_{n}\right)^{2}}
$$

and

$$
\frac{\partial F C_{n}}{\partial I_{n}}=-\frac{I_{i}}{\left(\sum_{n=1}^{N} I_{n}\right)^{2}} \text { for } n \neq i
$$

Substitution of the two derivatives into Eq. (4.1.2) gives

$$
\begin{array}{r}
\sigma_{F C_{i}}^{2}=\sigma_{I_{i}}^{2} \cdot\left[\frac{\sum_{n \neq i} I_{n}}{\left(\sum_{n=1}^{N} I_{n}\right)^{2}}\right]^{2}+\sum_{n \neq i} \sigma_{I_{n}}^{2}\left[\frac{I_{i}}{\left(\sum_{n=1}^{N} I_{n}\right)^{2}}\right]^{2} \\
=\frac{1}{\left(\sum_{n=1}^{N} I_{n}\right)^{4}}\left[\sigma_{I_{i}}^{2} \cdot\left(\sum_{n=1}^{N} I_{n}-I_{i}\right)^{2}+I_{i}^{2} \sum_{n \neq i} \sigma_{I_{n}}^{2}\right]
\end{array}
$$


The normalized integrated line intensities $\left(I_{n}{ }^{\prime}\right)$ and their corresponding errors are defined as:

$$
I_{n}^{\prime}=\frac{I_{n}}{\sum_{n=1}^{N} I_{n}}
$$

and

$$
\sigma_{I_{n}^{\prime}}=\frac{\sigma_{I_{n}}}{\sum_{n=1}^{N} I_{n}}
$$

Eq. (4.1.5) then becomes:

$$
\sigma_{F C_{i}}^{2}=\sigma_{I_{i}^{\prime}}^{2}\left(1-I_{i}^{\prime}\right)^{2}+\left(I_{i}^{\prime}\right)^{2} \sum_{n \neq i} \sigma_{I_{n}^{\prime}}^{2}
$$




\subsection{Vibronic Hamiltonian}

The well-known spin-free non-relativistic Hamiltonian of a molecule is:

$$
H=T_{n}+T_{e}+V_{n e}+V_{n n}+V_{e e}
$$

In static Born-Huang basis, the Hamiltonian takes a nice matrix form:

$$
H=\left[E_{\tilde{X}}+T_{n}\right] l+V
$$

The potential energy matrix is expanded in terms of dimensionless normal coordinate's $q_{i}$. In the past literature, generally Cartesian normal coordinates have been used to expand the potential energy matrix $V$ site papers here. We have used the cylindrical form of the normal coordinates for degenerate modes.

$$
\begin{gathered}
V=V_{1}+V_{2}+V_{3} \\
V_{1}=\left(\begin{array}{cccc}
0 & 0 & 0 & \cdots \\
0 & \Delta_{1} I_{n} & 0 & \cdots \\
0 & 0 & \Delta_{2} I_{n} & \cdots \\
\vdots & \vdots & \vdots & \ddots
\end{array}\right) \\
V_{2}=\frac{1}{2} I\left(\begin{array}{ccc}
n-p \\
\sum_{i}^{n} \omega_{i} q_{i}^{2}+\sum_{i}^{p} \omega_{i} q_{i+} q_{i-}
\end{array}\right)
\end{gathered}
$$




$$
V_{3}=\left(\begin{array}{cccc}
\sum_{i} \kappa_{i ; 0} q_{i} & \sum_{i} \lambda_{i}^{01} q_{i} & \sum_{i} \lambda_{i}^{02} q_{i} & \ldots \\
\sum_{i} \lambda_{i}^{01} q_{i} & \sum_{i} \kappa_{i ; 1} q_{i} & \sum_{i} \lambda_{i}^{12} q_{i} & \ldots \\
\sum_{i} \lambda_{i}^{02} q_{i} & \sum_{i} \lambda_{i}^{12} q_{i} & \sum_{i} \kappa_{i ; 2} q_{i} & \cdots \\
\vdots & \vdots & \vdots & \ddots
\end{array}\right)
$$

Diagonalization of $H$ gives the complete vibronic eigenvectors within the nonrelativistic framework. In the above equation, each electronic sub-block is further projected onto a basis of vibrational wave functions. In the case of no vibronic interactions, (the crude Born-Oppenheimer approximation), the hamiltonian $(H)$, Eq. (4.2.2) is block diagonal for each electronic state. It is to note that all the electronic states are expanded in the same complete set of normal coordinates, $q_{i}$, that are appropriate for the ground state. $I_{n}$ is an identity matrix of the same dimension as the truncated vibrational basis. $I$ is an identity matrix that spans the complete electronic $\otimes$ vibrational space.

Each element of this matrix spans the vibrational space and is too large to obtain it's eigenvalues by direct diagonalization without approximations. The pseudoJahn-Teller coupling between the $\tilde{X}$ and the $\tilde{A}$ state and the $\tilde{B}$ are pretty small and henceforth, will be ignored. The energy separation between the $\tilde{X}$ and the $\tilde{A}$ state is calculated, using EOMEE-CCSD/ccPVTZ, to be $15918 \mathrm{~cm}^{-1}$, compared to $2827 \mathrm{~cm}^{-1}$ between the $\tilde{A}$ and the $\tilde{B s t a t e}$ and thus, the pseudo-Jahn-Teller coupling between the $\tilde{A}$ and the $\tilde{B s h o u l d ~ d o m i n a t e . ~ T h e ~ c o r r e s p o n d i n g ~ e x p e r i m e n t a l ~} T_{0}$ values are $15925 \mathrm{~cm}^{-1}$ and 1757 
$\mathrm{cm}^{-1}$. By ignoring the pseudo-Jahn-Teller coupling between the $\tilde{X}$ and the $\tilde{A}$ and $\tilde{B}$ states we can block diagonalize this matrix and separate the $\tilde{X}$ states. The second approximation that we have made is that we have treated the pseudo-Jahn-Teller coupling between the $\tilde{A}$ and the $\tilde{B}$ states perturbatively, using the Van-Vleck transformation. This leads to the following simpler form of potential energy matrix $V$ :

$$
V=\left[\begin{array}{cc}
V_{\tilde{A}_{+}} & V_{\tilde{A}_{+-}} \\
V_{A_{-+}} & V_{\tilde{A}_{-}}
\end{array}\right]
$$

where,

$$
\begin{gathered}
V_{\tilde{A}+}=V_{\tilde{A}-}=\frac{1}{2} \sum_{i=1}^{4} \omega_{i}^{\prime} q_{i}^{2}+\frac{1}{2} \sum_{i=5}^{8} \omega_{i}^{\mathrm{eff}}\left(q_{i+} q_{i-}\right)+\sum_{i=1}^{4} d_{i} q_{i} \\
\omega_{i}^{\mathrm{eff}}=\omega_{i}+\Delta_{\omega_{i}} \\
V_{\tilde{A}_{+-}=}=\sum_{i=5}^{8} k_{i} q_{i-}+\sum_{i=5}^{8} g_{i i}^{\mathrm{eff}} q_{i+}^{2}+\sum_{i=5}^{7} \sum_{j=i+1}^{8} g_{i j} q_{i+} q_{j+}+\sum_{i=1}^{4} \sum_{j=5}^{8} b_{i j} q_{i} q_{j-} \\
g_{i i}^{\mathrm{eff}}=g_{i i}+\Xi_{i}^{A B}
\end{gathered}
$$




$$
\begin{gathered}
V_{\alpha \beta}=\sum_{i=1}^{4} \lambda_{i}^{\alpha \beta} q_{i}+\sum_{i=5}^{8} \lambda_{i}^{\alpha \beta} q_{i-} \\
\Delta_{\omega_{i}}=\frac{\left(\lambda_{i}^{A_{a} B}\right)^{2}+\left(\lambda_{i}^{A_{b} B}\right)^{2}}{\left(E_{\tilde{A}}-E_{\tilde{B}}\right)} \\
\Xi_{i}^{A B}=\frac{\left(\lambda_{i}^{A_{a} B}\right)^{2}-\left(\lambda_{i}^{A_{b} B}\right)^{2}}{\left(E_{\tilde{A}}-E_{\tilde{B}}\right)}
\end{gathered}
$$$$
\text { ) }
$$

By including the nuclear kinetic energy (excluding rotation) we can define the classic JahnTeller Hamiltonian to second order

$$
H_{J T}=T_{n}+V
$$

The conventional harmonic frequencies $\left(\omega_{i}\right)$ and Jahn-Teller coupling coefficients $k_{i}, g_{i j}$ and $b_{i j}$ are related to the derivative of adiabatic electronic potential energy surface ( $\mathcal{C}$ ) as described in Table S.1. The pseudo Jahn Teller coupling between $A^{\tilde{N}}$ and $B^{\tilde{B}}$ states is defined as

$$
\begin{aligned}
\lambda_{i}^{A_{a} B} & =\left.\frac{\partial}{\partial q_{i}}\left(\left\langle\Lambda_{A ; a}\left|\mathscr{H}_{e}\right| \Lambda_{B}\right\rangle\right)\right|_{R_{0}} \\
\lambda_{i}^{A_{b} B} & =\left.\frac{\partial}{\partial q_{i}}\left(\left\langle\Lambda_{A ; b}\left|\mathscr{H}_{e}\right| \Lambda_{B}\right\rangle\right)\right|_{R_{0}}
\end{aligned}
$$


The second-order contribution obtained by the pseudo-Jahn-Teller coupling of the $\tilde{A}$ and $\tilde{B}$ are also included in the effective vibrational frequency $\omega_{i}^{\text {eff }}$ and the quadratic Jahn-Teller coupling constant $g_{i i}$. Similar corrections due to the smaller cross quadratic and bilinear couplings (although negligible) are also included.

We use CFOUR (quantum chemistry package) to calculate these coefficients. CFOUR uses Cartesian representation for degenerate components of electronic states and degenerates normal modes. The two degenerate components of the $\tilde{A}^{2} E$ electronic state are distinguished as they transform differently in the $C_{s}$ symmetry group, an abelian subgroup of the $C_{3 v}$ group, with one component transforming as $A^{\prime}$ irreducible representation and the other as $A^{\prime \prime}$. The relation between the Cartesian and cylindrical representations is:

$$
\begin{gathered}
\left|\Lambda_{ \pm}\right\rangle=\frac{1}{\sqrt{2}}\left[\left|\Lambda_{A ; a}\right\rangle \pm i\left|\Lambda_{A ; b}\right\rangle\right] \\
q_{i \pm}=q_{i a} \pm i q_{i b}
\end{gathered}
$$

We calculate the intrastate and interstate coupling constants by perturbing along the totally symmetric mode $\left(A^{\prime}\right)$ and then use finite differences to calculate first and second derivatives. Table 4.2 lists the values of harmonic frequency, linear and quadratic JahnTeller coupling constants, $\kappa_{i}$ and $g_{i i}$, the pseudo-Jahn-Teller coupling constant for the coupling between $\tilde{A}$ and $\tilde{B}$ states, $\Xi_{i}$ and the correction to harmonic frequency $\Delta_{\omega i}$. 


\subsection{Intensity calculation}

The eigenvalues and eigenvectors of the spin-vibronic Hamiltonian are obtained using SOCJT 2. The spin-vibronic eigenvectors, when taking Jahn-Teller coupling into account, are of the form

$$
\left|\Psi_{\tilde{A}}^{n}\right\rangle=\sum_{i} c_{i}^{n}\left|\Lambda_{A ; i}\right\rangle \prod_{k=1}^{4}\left|v_{k ; A}^{i}\right\rangle \prod_{k=5}^{8}\left|v_{k, A}^{i}, l_{k, A}^{i}\right\rangle\left|S, \Sigma_{i}\right\rangle
$$

The index $n$ enumerates the eigenvectors in increasing order of energy. Table S.5 gives the energy and labels of the states of relevance.

So the dipole moment for the transition between the $n^{\text {th }} \tilde{A}$ state spin-vibronic level and the $\tilde{X}$ state vibrationless level would be given by

$f\left\langle\Psi_{\tilde{X}}\left|\hat{\mu}_{\alpha}\right| \Psi_{\tilde{A}}^{n}\right\rangle=\sum_{i} c_{i}^{n}\left\langle\Lambda_{X}\left|\prod_{k=1}^{4}\left\langle v_{k ; X}\left|\prod_{k=5}^{8}\left\langle v_{k, X}, l_{k, X}\left|\hat{\mu}_{\alpha}\right| \Lambda_{A}\right\rangle \prod_{k}^{n-p}\right| v_{i ; A}\right\rangle \prod_{k}^{p}\right| v_{i, A}, l_{i, A}\right\rangle$

When the pseudo-Jahn-Teller term was eliminated from the matrix, to create the matrix in Eq. (S.3.7), the latter's basis set was altered to include a contribution from the $\tilde{B \text { state. }}$ Including this contribution gives us the complete transition moment:

$$
\left\langle\Psi_{X 0_{0}}\left|\hat{\mu}_{\alpha}\right| \Psi_{\tilde{A}}^{n}\right\rangle=\left.c_{0}^{n}\left\langle\Lambda_{X}\left|\hat{\mu}_{\alpha}\right| \Lambda_{A ; i}\right\rangle\right|_{R_{0}}+\sum_{a} \frac{\left.c_{1 a}^{n} \lambda_{a}^{A_{i(a)} B}\left\langle\Lambda_{X}\left|\hat{\mu}_{\alpha}\right| \Lambda_{B}\right\rangle\right|_{R_{0}}}{\sqrt{2}\left(E_{\Lambda_{B}}-E_{\Lambda_{A}}\right)}
$$


where $c_{0}$ is the coefficient of the vibrationless basis function and $c_{1 \alpha}$ is the coefficient of the term

$$
\left|v_{\alpha}=1\right\rangle \prod_{\substack{k=1 \\ k \neq \alpha}}^{3 N-6}\left|v_{k}=0\right\rangle
$$

The vibronic eigenvectors are obtained using SOCJT2. The transition dipole matrix elements are calculated using EOMEE-CCSD/cc-pVTZ. Table S.7 lists the values of the electronic transition dipole matrix element. Table 4.8-Table 4.13 presents in detail, the intensity calculations for both excitation and emission spectra using this formulation. 


\subsection{Vibronic and Spin-Orbit interactions between the $\widetilde{B}^{2} A_{1}$ and the $\widetilde{A}^{2} E$ states}

The SO Hamiltonian can be written

$$
H_{S O}=a_{\|} L_{z} S_{z}+a_{\perp}\left(L_{x} S_{x}+L_{y} S_{y}\right)
$$

If we introduce the spherical tensor operators

$$
T_{0}^{1}(L)=L_{z} \text { and } T_{ \pm 1}^{1}(L)=\mp \frac{1}{\sqrt{2}}\left(L_{x} \pm i L_{y}\right)
$$

and the same for $S$ then

$$
H_{S O}=a_{\|} T_{0}^{1}(L) T_{0}^{1}(S)+a_{\perp} \sum_{q= \pm 1}(-1)^{q} T_{q}^{1}(L) T_{-q}^{1}(S)
$$

does not couple an $E$ and an $A$ state for symmetry reasons so we neglect it and we write the perturbing Hamiltonian

$$
H_{p}=a_{\perp} \sum_{q= \pm 1}(-1)^{q} T_{q}^{1}(L) T_{-q}^{1}(S)
$$

Using the Wigner-Eckart theorem, the product basis set, and the hypothesis of pure precession

$$
\begin{aligned}
& \left\langle L=1 \Lambda \pm 1\left|T_{ \pm 1}^{1}(L)\right| L=1 \Lambda\right\rangle=\mp 1 \\
& \left\langle S=\frac{1}{2}, \Sigma \pm 1\left|T_{ \pm 1}^{1}(S)\right| S=\frac{1}{2}, \Sigma\right\rangle=\frac{\mp 1}{\sqrt{2}}
\end{aligned}
$$


Therefore the two required equivalent matrix elements of the sum in Eq. (4.4) are of the form

$$
(-1)^{q}\left\langle 1, \pm 1\left|T_{\mp 1}^{1}(L)\right| 1,0\right\rangle\left\langle\frac{1}{2}, \mp \frac{1}{2}\left|T_{ \pm 1}^{1}(S)\right| \frac{1}{2}, \pm \frac{1}{2}\right\rangle=\frac{1}{\sqrt{2}}
$$

with $\mathrm{q}= \pm 1$. Hq, therefore, couples the $\tilde{A}^{2} E$ and $\tilde{B}^{2} A_{1}$ levels with the same $\mathrm{v}$ and for which $\Lambda$ and $\Sigma$ are aligned anti-parallel. Note that there is no coupling between the $\tilde{B}$ state and the

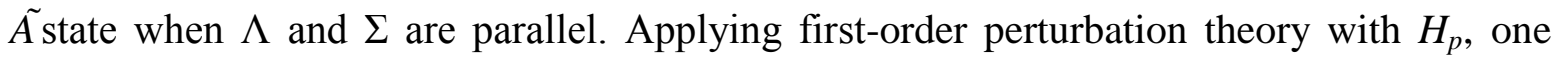
reaches Eq. (2) and Eq.(3) for mixing between the $\tilde{A a n d} \tilde{B}$ wavefunction via (linear) pJT coupling. 
Table 4.1: Harmonic frequency and Jahn Teller coupling parameters in cylindrical coordinates. $\mathcal{H}_{e}$ is the electronic potential at the symmetric configuration, which includes Coulomb interaction, exchange interaction etc.

\begin{tabular}{|c|c|c|}
\hline Symbols & Description & Definition \\
\hline$d_{i}$ & Gradient along symmetric mode & $\left.\frac{\partial}{\partial q_{i}}\left(\left\langle\Lambda_{ \pm}\left|\mathscr{H}_{e}\right| \Lambda_{ \pm}\right\rangle\right)\right|_{R_{0}}$ \\
$k_{i}$ & Linear JT coupling constant & $\left.\frac{\partial}{\partial q_{i \pm}}\left(\left\langle\Lambda_{ \pm}\left|\mathscr{H}_{e}\right| \Lambda_{\mp}\right\rangle\right)\right|_{R_{0}}$ \\
$\omega_{i}$ & Harmonic force constant for JT active mode & $\left.\frac{\partial^{2}}{\partial q_{i,+} \partial q_{i,-}}\left(\left\langle\Lambda_{ \pm}\left|\mathscr{H}_{e}\right| \Lambda_{ \pm}\right\rangle\right)\right|_{R_{0}}$ \\
$\omega_{i}^{\prime}$ & Harmonic force constant for JT inactive mode & $\left.\frac{\partial^{2}}{\partial q_{j}^{2}}\left(\left\langle\Lambda_{ \pm}\left|\mathscr{H}_{e}\right| \Lambda_{ \pm}\right\rangle\right)\right|_{R_{0}}$ \\
$g_{i i}$ & Quadratic JT coupling constant & $\left.\frac{\partial^{2}}{\partial q_{i \pm \pm}^{2}}\left(\left\langle\Lambda_{ \pm}\left|\mathscr{H}_{e}\right| \Lambda_{\mp}\right\rangle\right)\right|_{R_{0}}$ \\
$g_{i j}$ & Cross-Quadratic JT coupling constant & $\left.\frac{\partial^{2}}{\partial q_{i, \pm} \partial q_{j, \pm}}\left(\left\langle\Lambda_{ \pm}\left|\mathscr{H}_{e}\right| \Lambda_{\mp}\right\rangle\right)\right|_{R_{0}}$ \\
$b_{i j}$ & Bilinear JT coupling constant & $\left.\frac{\partial^{2}}{\partial q_{i, p m} \partial q_{j}}\left(\left\langle\Lambda_{ \pm}\left|\mathscr{H}_{e}\right| \Lambda_{\mp}\right\rangle\right)\right|_{R_{0}}$ \\
\hline
\end{tabular}




\begin{tabular}{|c|c|c|}
\hline$\subseteq$ & $\stackrel{5}{\square}$ & શે ले సે \\
\hline 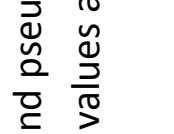 & 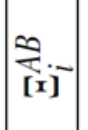 & 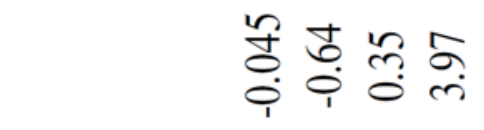 \\
\hline 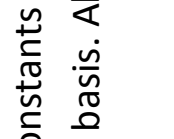 & 年 & 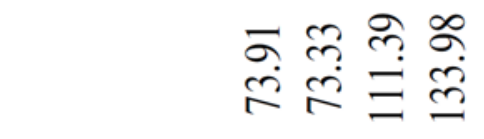 \\
\hline 㤎 & - & 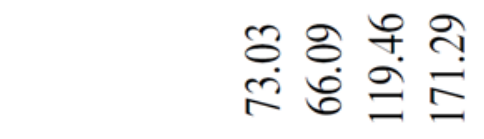 \\
\hline 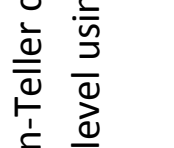 & $: \approx$ & 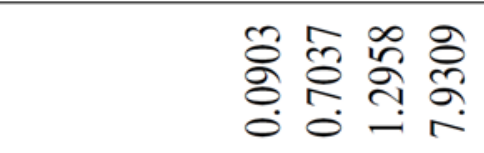 \\
\hline 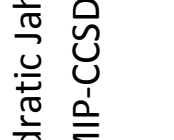 & $\therefore$ & 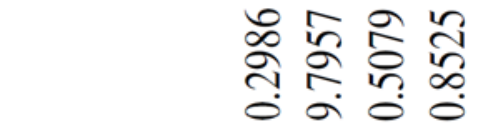 \\
\hline 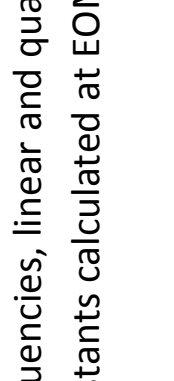 & 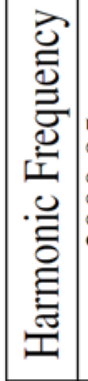 & 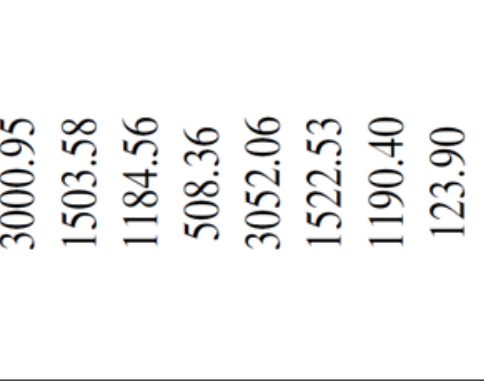 \\
\hline 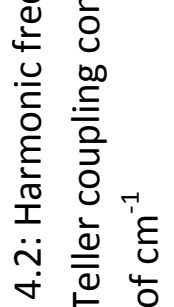 & 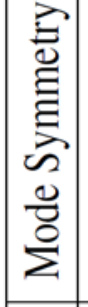 & E $\bar{E}$ E 00 \\
\hline 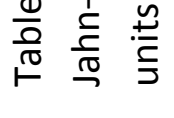 & $\sum_{0}^{\frac{0}{2}}$ & 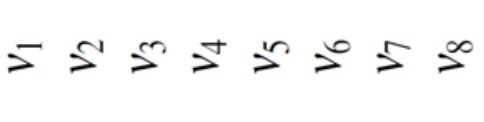 \\
\hline
\end{tabular}


Table 4.3: Cross-quadratic coupling constants calculated at EOMIP-CCSD level using cc- pVTZ basis. All values are in units of $\mathrm{cm}^{-1}$.

\begin{tabular}{|c|c|c|}
\hline Mode & Mode & $g_{i j}$ \\
\hline$v_{5}$ & $v_{6}$ & 0.63 \\
$v_{5}$ & $v_{7}$ & 0.09 \\
$v_{5}$ & $v_{8}$ & -10.30 \\
$v_{6}$ & $v_{7}$ & -0.46 \\
$v_{6}$ & $v_{8}$ & -0.03 \\
$v_{7}$ & $v_{8}$ & -1.14 \\
\hline
\end{tabular}

Table 4.4: Bilinear coupling constants calculated at EOMIP-CCSD level using cc-pVTZ basis. All values are in units of $\mathrm{cm}^{-1}$.

\begin{tabular}{|c|c|c|}
\hline Mode & Mode & $b_{i j}$ \\
\hline$v_{5}$ & $v_{1}$ & 0.38 \\
$v_{5}$ & $v_{2}$ & 0.30 \\
$v_{5}$ & $v_{3}$ & 0.15 \\
$v_{5}$ & $v_{4}$ & 0.28 \\
$v_{6}$ & $v_{1}$ & -0.07 \\
$v_{6}$ & $v_{2}$ & -0.86 \\
$v_{6}$ & $v_{3}$ & -0.50 \\
$v_{6}$ & $v_{4}$ & 1.89 \\
$v_{7}$ & $v_{1}$ & 0.03 \\
$v_{7}$ & $v_{2}$ & 0.04 \\
$v_{7}$ & $v_{3}$ & -0.02 \\
$v_{7}$ & $v_{4}$ & 0.35 \\
$v_{8}$ & $v_{1}$ & 0.33 \\
$v_{8}$ & $v_{2}$ & -0.38 \\
$v_{8}$ & $v_{3}$ & -0.03 \\
$v_{8}$ & $v_{4}$ & 0.14 \\
\hline
\end{tabular}




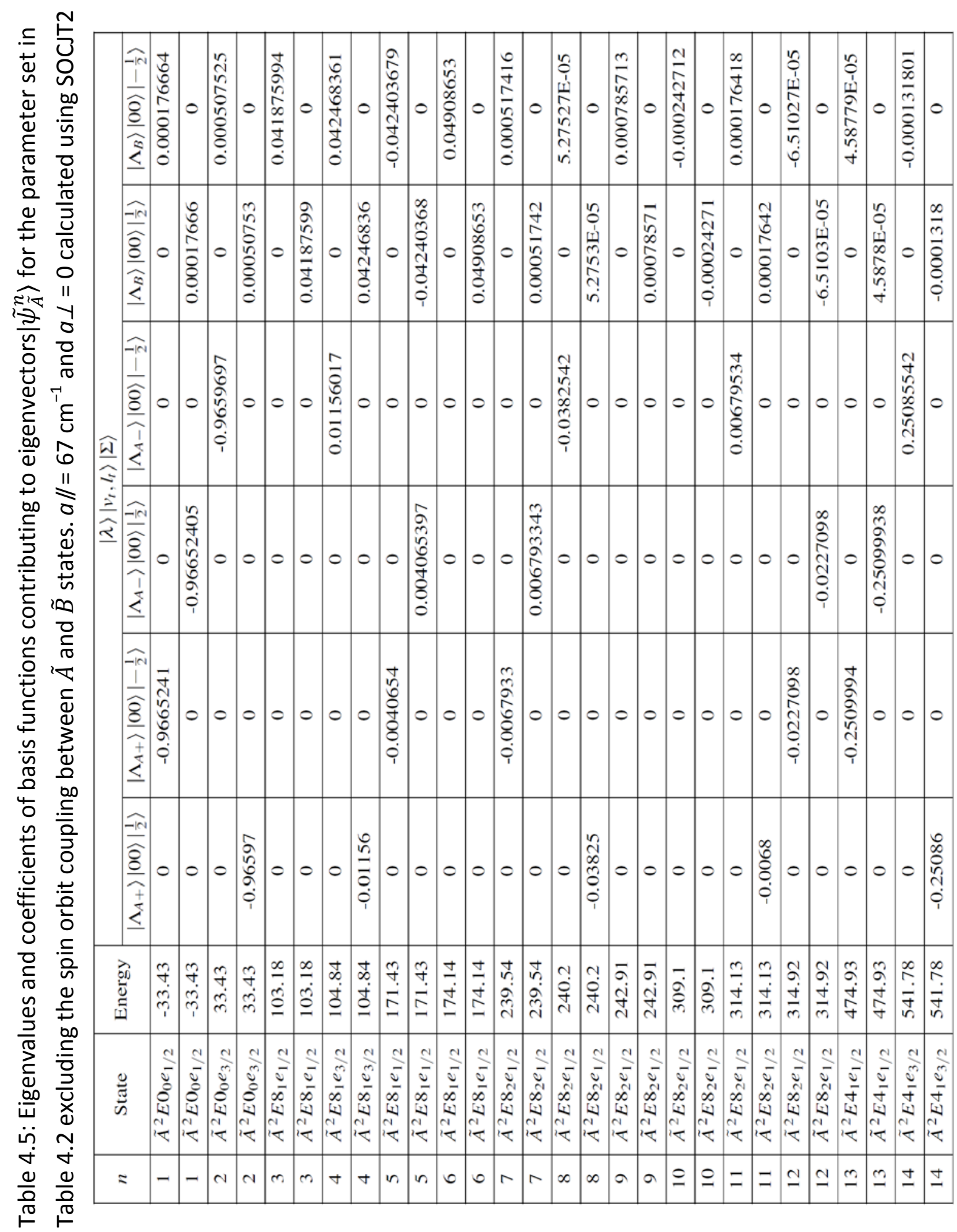




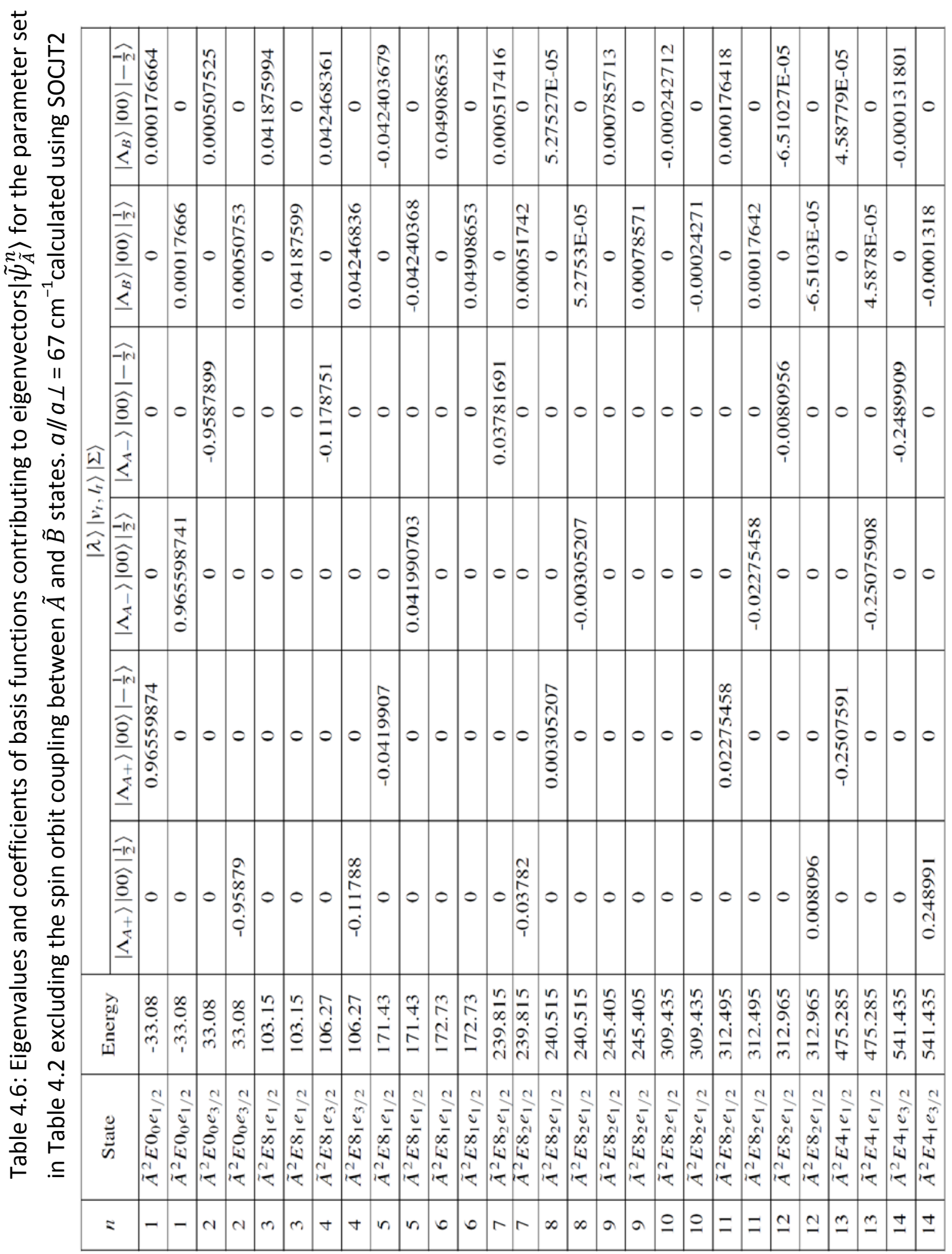


Table 4.7: Electronic Transition Dipole Moment from $\Lambda_{X}$ to $\Lambda_{A}$ and $\Lambda_{B}$ states

\begin{tabular}{|c|c|}
\hline & Electronic Transition Dipole moment (in Debye) \\
\hline$\left\langle\Lambda_{X}\left|\mu_{x}\right| \Lambda_{A ; a}\right\rangle$ & 0 \\
\hline$\left\langle\Lambda_{X}\left|\mu_{y}\right| \Lambda_{A ; a}\right\rangle$ & 2.3728 \\
\hline$\left\langle\Lambda_{X}\left|\mu_{z}\right| \Lambda_{A ; a}\right\rangle$ & 0 \\
\hline$\left\langle\Lambda_{X}\left|\mu_{x}\right| \Lambda_{A ; b}\right\rangle$ & 2.3728 \\
\hline$\left\langle\Lambda_{X}\left|\mu_{y}\right| \Lambda_{A ; b}\right\rangle$ & 0 \\
\hline$\left\langle\Lambda_{X}\left|\mu_{z}\right| \Lambda_{A ; b}\right\rangle$ & 0 \\
\hline$\left\langle\Lambda_{X}\left|\mu_{x}\right| \Lambda_{B}\right\rangle$ & 0 \\
\hline$\left\langle\Lambda_{X}\left|\mu_{y}\right| \Lambda_{B}\right\rangle$ & 0 \\
\hline$\left\langle\Lambda_{X}\left|\mu_{z}\right| \Lambda_{B}\right\rangle$ & 1.9241 \\
\hline
\end{tabular}




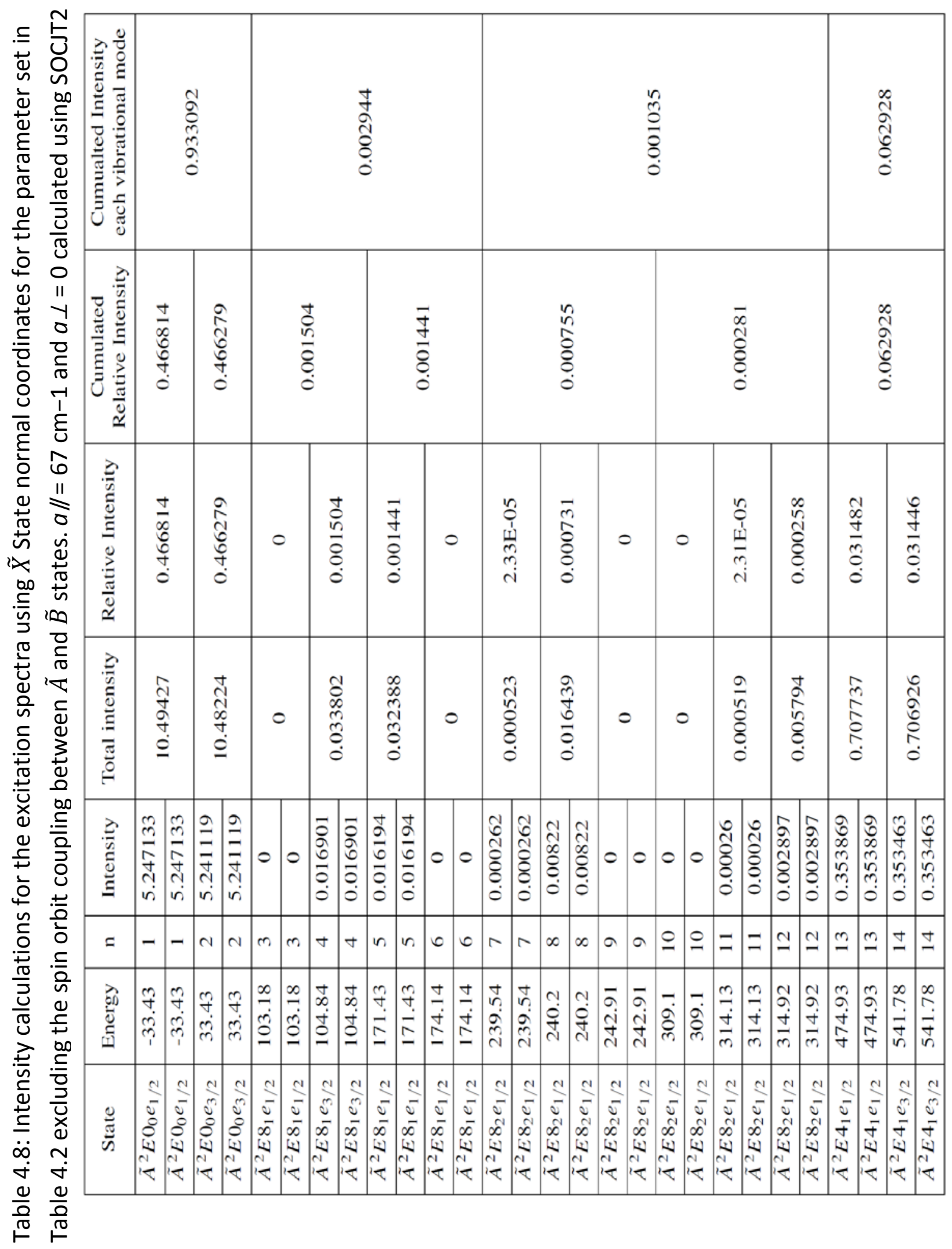


Table 4.9: Intensity calculations for $\tilde{X} \leftarrow \tilde{A} 0_{0} E_{1 / 2}$ emission spectra using $\tilde{X}$ state normal coordinates for the parameter set in Table 5.2 excluding the spin orbit coupling between $\tilde{A}$ and $\tilde{B}$ states. $a / /=67 \mathrm{~cm}-1$ and $a \perp=0$ calculated using SOCJT2

\begin{tabular}{|c|c|c|c|c|}
\hline State & Energy & $\left|\left\langle\tilde{X}^{2} E a_{b}|\mu| \tilde{A}^{2} E 0_{0}\right\rangle\right|^{2}$ & $\begin{array}{c}\text { Normalized } \\
\text { Transition Moment }\end{array}$ & $\begin{array}{c}\text { Relative } \\
\text { Intensity }\end{array}$ \\
\hline$\tilde{X}^{2} A_{1} 0_{0}$ & 0 & 10.49427 & 0.933677 & 0.9378 \\
\hline$\tilde{X}^{2} A_{1} 8_{1}$ & 123.90 & 0.032574 & 0.002898 & 0.0028 \\
\hline$\tilde{X}^{2} A_{1} 8_{2}$ & 247.81 & 0.00555 & 0.000494 & 0.0005 \\
\hline$\tilde{X}^{2} A_{1} 4_{1}$ & 508.36 & 0.707332 & 0.062931 & 0.0573 \\
\hline$\tilde{X}^{2} A_{1} 4_{2}$ & 1016.716 & 0.023865 & 0.001836 & 0.0015 \\
\hline
\end{tabular}


Table 4.10: Intensity calculations for $\tilde{X} \leftarrow \tilde{A} 0_{0} E_{3 / 2}$ emission spectra using $\tilde{X}$ state normal coordinates for the parameter set in Table 5.2 excluding the spin orbit coupling between $\tilde{A}$ and $\tilde{B}$ states. $a / /=67 \mathrm{~cm}^{-1}$ and $a \perp=0$ calculated using SOCJT2

\begin{tabular}{|c|c|c|c|c|}
\hline State & Energy & $\left|\left\langle\tilde{X}^{2} E a_{b}|\mu| \tilde{A}^{2} E 0_{0}\right\rangle\right|^{2}$ & $\begin{array}{c}\text { Normalized } \\
\text { Transition Moment }\end{array}$ & $\begin{array}{c}\text { Relative } \\
\text { Intensity }\end{array}$ \\
\hline$\tilde{X}^{2} E 0_{0}$ & 0 & 10.48224 & 0.932722 & 0.9369 \\
\hline$\tilde{X}^{2} E 8_{1}$ & 123.90 & 0.033884 & 0.003015 & 0.0030 \\
\hline$\tilde{X}^{2} E 8_{2}$ & 247.81 & 0.01488 & 0.001324 & 0.0013 \\
\hline$\tilde{X}^{2} E 4_{1}$ & 508.36 & 0.707332 & 0.062939 & 0.0574 \\
\hline$\tilde{X}^{2} E 4_{2}$ & 1016.716 & 0.023838 & 0.001834 & 0.0015 \\
\hline
\end{tabular}




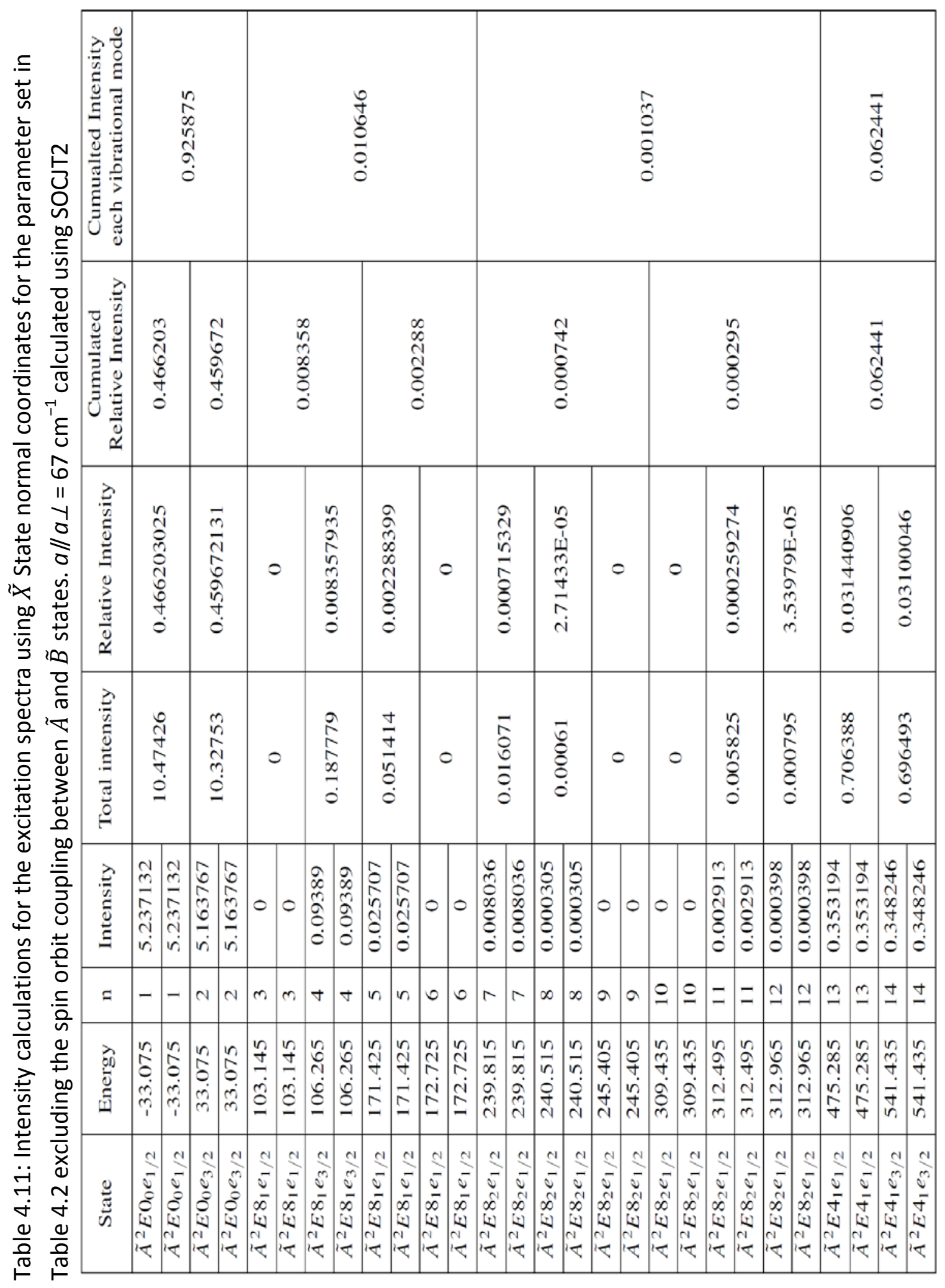


Table 4.12: Intensity calculations for $\tilde{X} \leftarrow \tilde{A} 0_{0} E_{1 / 2}$ emission spectra using $\tilde{X}$ state normal coordinates for the parameter set in Table 4.2 excluding the spin orbit coupling between $\tilde{A}$ and $\tilde{B}$ states. $a \| a \perp=67 \mathrm{~cm}^{-1}$ calculated using SOCJT2

\begin{tabular}{|c|c|c|c|c|}
\hline State & Energy & $\left|\left\langle\tilde{X}^{2} E a_{b}|\mu| \tilde{A}^{2} E 0_{0}\right\rangle\right|^{2}$ & $\begin{array}{c}\text { Normalized } \\
\text { Transition Moment }\end{array}$ & $\begin{array}{c}\text { Relative } \\
\text { Intensity }\end{array}$ \\
\hline$\tilde{X}^{2} A_{1} 0_{0}$ & 0 & 10.47426 & 0.93249 & 0.0 .9363 \\
\hline$\tilde{X}^{2} A_{1} 8_{1}$ & 123.90 & 0.051876 & 0.004618 & 0.0045 \\
\hline$\tilde{X}^{2} A_{1} 8_{2}$ & 247.81 & 0.00499 & 0.000444 & 0.0004 \\
\hline$\tilde{X}^{2} A_{1} 4_{1}$ & 508.36 & 0.701441 & 0.062447 & 0.0573 \\
\hline$\tilde{X}^{2} A_{1} 4_{2}$ & 1016.716 & 0.023819 & 0.001832 & 0.0015 \\
\hline
\end{tabular}


Table 4.13: Intensity calculations for $\tilde{X} \leftarrow \tilde{A} 0_{0} E_{3 / 2}$ emission spectra using $\tilde{X}$ state normal coordinates for the parameter set in Table 4.2 excluding the spin orbit coupling between $\tilde{A}$ and $\tilde{B}$ states. $a \| a \perp=67 \mathrm{~cm}^{-1}$ calculated using SOCJT2

\begin{tabular}{|c|c|c|c|c|}
\hline State & Energy & $\left|\left\langle\tilde{X}^{2} E a_{b}|\mu| \tilde{A}^{2} E 0_{0}\right\rangle\right|^{2}$ & $\begin{array}{c}\text { Normalized } \\
\text { Transition Moment }\end{array}$ & $\begin{array}{c}\text { Relative } \\
\text { Intensity }\end{array}$ \\
\hline$\tilde{X}^{2} A_{1} 0_{0}$ & 0 & 10.32753 & 0.919728 & 0.9241 \\
\hline$\tilde{X}^{2} A_{1} 8_{1}$ & 123.90 & 0.187506 & 0.016699 & 0.0163 \\
\hline$\tilde{X}^{2} A_{1} 8_{2}$ & 247.81 & 0.01242 & 0.001106 & 0.0010 \\
\hline$\tilde{X}^{2} A_{1} 4_{1}$ & 508.36 & 0.701441 & 0.062467 & 0.0570 \\
\hline$\tilde{X}^{2} A_{1} 4_{2}$ & 1016.716 & 0.023485 & 0.001807 & 0.0015 \\
\hline
\end{tabular}




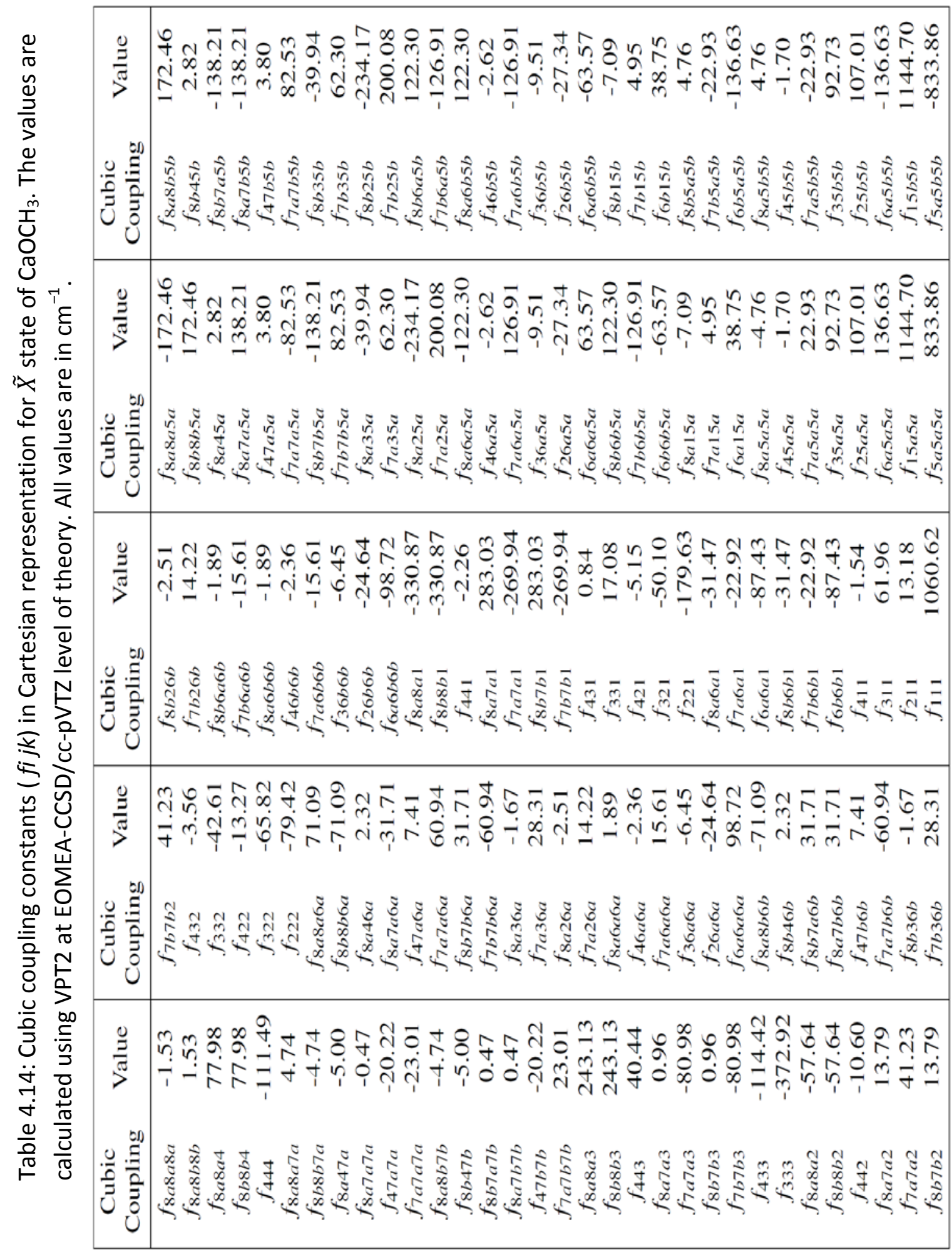




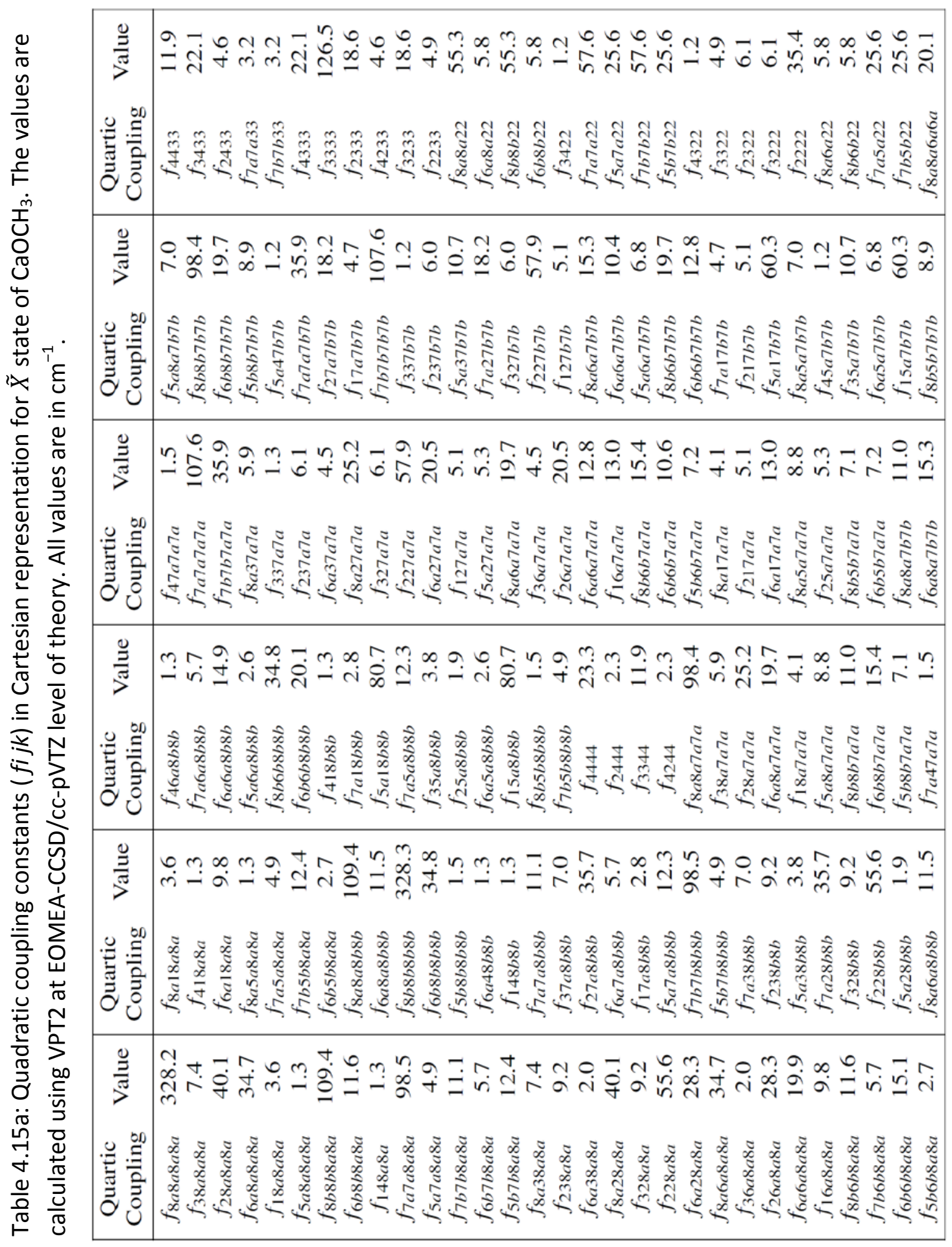




\begin{tabular}{|c|c|c|}
\hline & 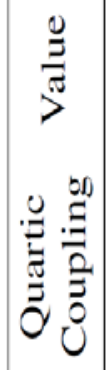 & 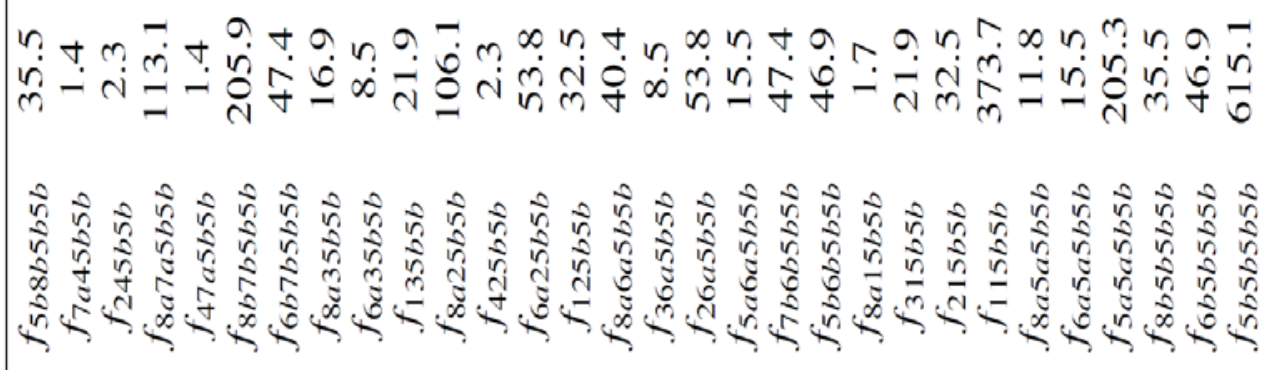 \\
\hline$\pi$ & 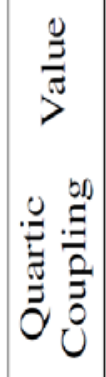 & 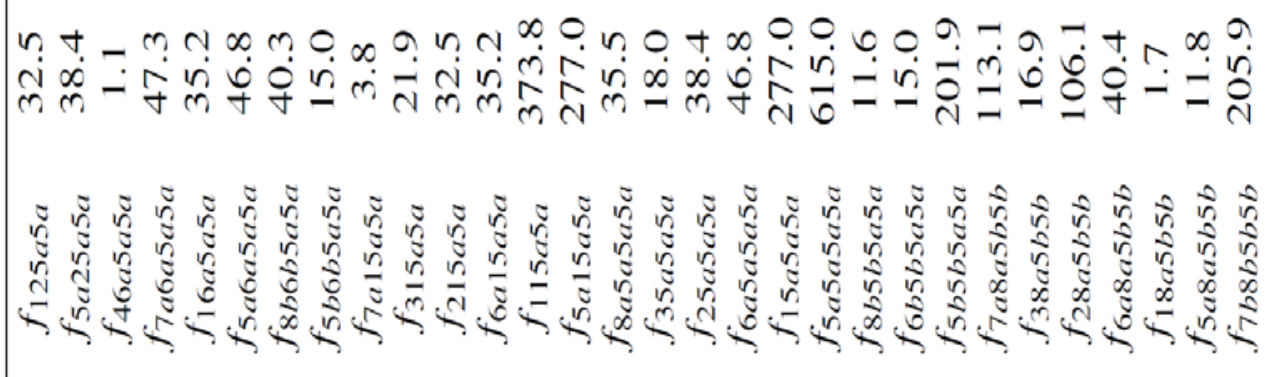 \\
\hline$\overline{\overline{\bar{\alpha}}}$ & 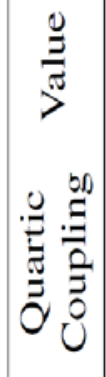 & 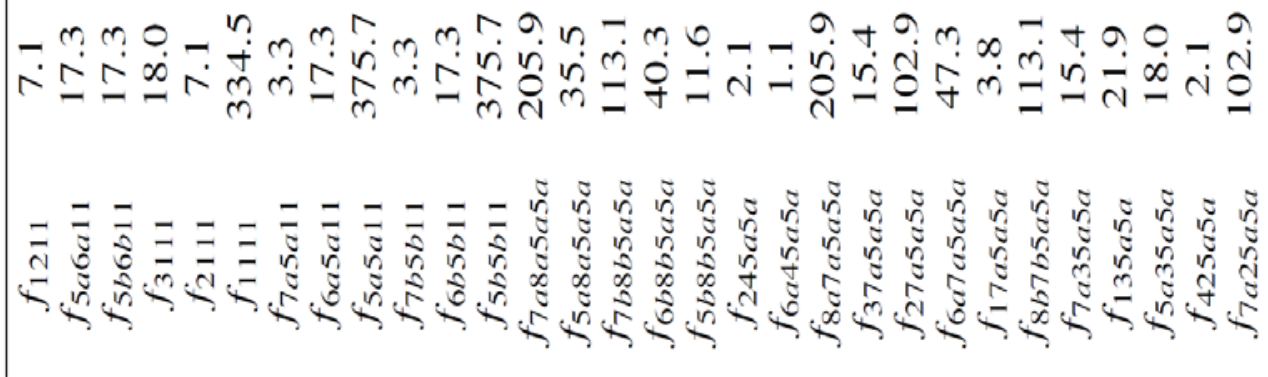 \\
\hline 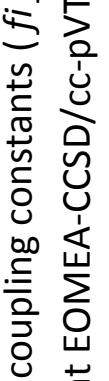 & 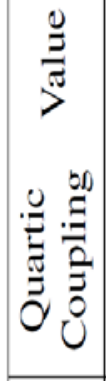 & 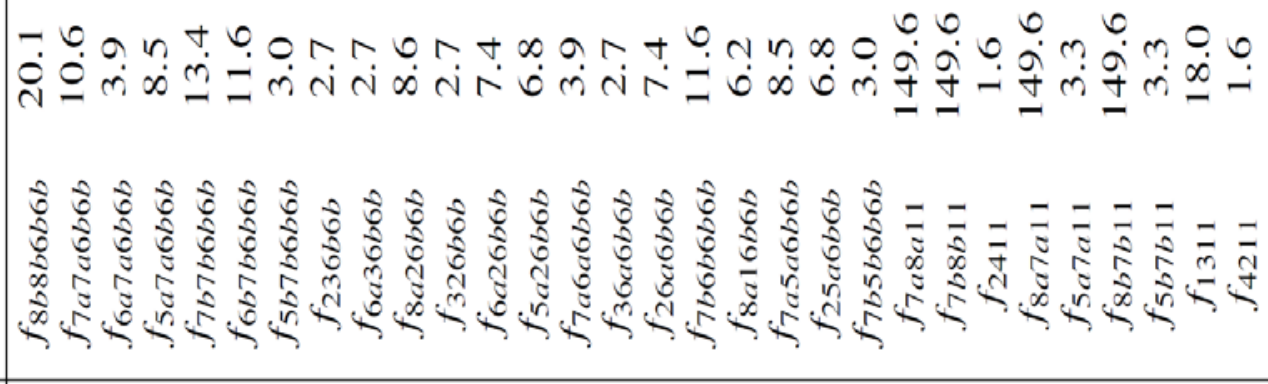 \\
\hline$\frac{2}{a}$ & 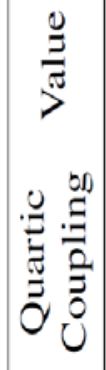 & 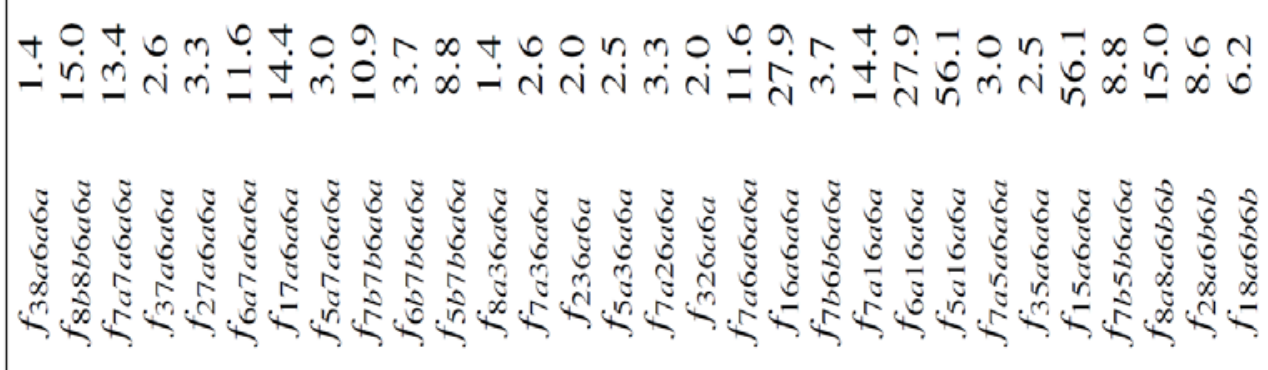 \\
\hline
\end{tabular}


Table 4.16: Frank Condon factors $f v^{\prime} v^{\prime \prime}=\left|\left\langle v^{\prime} \mid v^{\prime \prime}\right\rangle\right|^{2}$ for $\tilde{A} \leftarrow \tilde{X} 0_{0}$ transition

\begin{tabular}{|c|c|}
\hline Transition & $f_{v^{\prime} v^{\prime \prime}}$ \\
\hline $0^{0}$ & 0.928265 \\
$8 a^{1}$ & 0 \\
$8 b^{1}$ & 0 \\
$8 a^{2}$ & $2.166117 \mathrm{E}-05$ \\
$8 a^{1} 8 b^{1}$ & 0 \\
$8 b^{2}$ & $2.166117 \mathrm{E}-05$ \\
$4^{1}$ & 0.064009 \\
$4^{2}$ & $1.980168 \mathrm{E}-03$ \\
$3^{1}$ & $4.473643 \mathrm{E}-03$ \\
$4^{3}$ & $3.593974 \mathrm{E}-05$ \\
$2^{1}$ & $6.264468 \mathrm{E}-04$ \\
\hline
\end{tabular}

Table 5.17: Frank Condon factors $f v^{\prime} v^{\prime \prime}=\left|\left\langle v^{\prime} \mid v^{\prime \prime}\right\rangle\right|^{2}$ for $\tilde{X} \leftarrow \tilde{A} 0_{0}$ transition

\begin{tabular}{|c|c|}
\hline Transition & $f_{v^{\prime} v^{\prime \prime}}$ \\
\hline $0^{0}$ & 0.928265 \\
$8 a^{1}$ & 0 \\
$8 b^{1}$ & 0 \\
$8 a^{2}$ & $2.2186 \mathrm{E}-05$ \\
$8 a^{1} 8 b^{1}$ & 0 \\
$8 b^{2}$ & $2.2186 \mathrm{E}-05$ \\
$4^{1}$ & 0.063628 \\
$4^{2}$ & $2.418058 \mathrm{E}-03$ \\
$3^{1}$ & $4.495803 \mathrm{E}-03$ \\
$4^{3}$ & $6.693465 \mathrm{E}-05$ \\
$2^{1}$ & $2.218604 \mathrm{E}-05$ \\
\hline
\end{tabular}




\section{CHAPTER V}

\section{LASER-INDUCED FLUORESCENCE AND DISPERSED FLUORESCENCE SPECTROSCOPY OF JET-COOLED}

\section{CALCIUM ETHOXIDE}

Metal-containing free radicals are important intermediates in metal-surface reactions and interactions between metals and organic molecules. Among these metalcontaining radicals, monovalent derivatives of alkaline earth metals have been well studied spectroscopically in the gas phase ${ }^{2,29,66,67}$ (in Broida ovens ${ }^{28}$ or under supersonic jet-cooled conditions. They are of special interest to theoretical molecular spectroscopists too. Many such molecules, including the organometallic monomethyl radicals such as $\mathrm{MgCH}_{3}{ }^{92}, \mathrm{CaCH}_{3}{ }^{93}$, and $\mathrm{SrCH}_{3}{ }^{93}$, as well as their corresponding methoxide radicals $\left(\mathrm{MgOCH}_{3}, \mathrm{CaOCH}_{3}{ }^{48,71}\right.$, and $\left.\mathrm{SrOCH}_{3}{ }^{94}\right)$, have $\mathrm{C}_{3 v}$ symmetry and their first electronically excited $\left(\tilde{A}^{2} E\right)$ state would be orbitally degenerate if there were no spin-vibronic interactions. The $\tilde{A}^{2} E$ state, however, is subject to spin-orbit (SO) and Jahn-Teller (JT) interactions, both of which remove the degeneracy. Its potential energy surface (PES) is distorted by the JT interaction and further split by the SO interaction into two components, $E_{1 / 2}$ and $E_{3 / 2}$. Because both interactions compete for the orbital angular momentum, the SO splitting between the $\mathrm{E}_{1 / 2}$ and $\mathrm{E}_{3 / 2}$ states is quenched by the JT effect ${ }^{95}$. 
For alkaline earth molecules, the molecular symmetry is lowered from $\mathrm{C}_{3 v}$ to $\mathrm{C}_{\mathrm{s}}$ or $\mathrm{C}_{1}$ and the JT effect is reduced to pseudo-Jahn-teller (pJT) effect. In the $\mathrm{C}_{\mathrm{s}}$ symmetry case, the $\tilde{A}^{2} E$ state of organometallic monomethyl radicals is split into two states with $A^{\prime}$ and $A^{\prime \prime}$ symmetries. The energy separation between these two states is determined by SO and vibronic interactions, as well as the difference between their zeropoint energies (ZPEs) ${ }^{96,97}$. The vibronic structures of the pJT molecules may also be significantly different from those of the JT ones. Historically, energy level structure and intramolecular dynamics of JT-active molecules have been studied extensively by molecular spectroscopy, with both vibronic or even rotational resolution ${ }^{80,86}$. There are, however, much fewer spectroscopic works on the pJT molecules. Bernath and coworkers first reported laser-induced fluorescence (LIF) and dispersed fluorescence (DF) spectra of alkaline earth monoalkoxide radicals (M-O-R; M = Ca, Sr, and Ba). These radicals were made in a Broida-type oven by the reaction of a metal vapor with an oxidant (alcohol, acetone, or acetaldehyde). The observed DF spectra revealed the ground-state vibrational structure, while the energy separation between the nearly degenerate $\tilde{A}_{1}$ and $\tilde{A}_{2}$ states was directly measured in the LIF experiment. In Chapter 3 we reported the LIF and DF of $\mathrm{CaOCH}_{3}$ which reveals clearly the vibronic structure of it. In the present chapter LIF and DF spectra of $\mathrm{CaOCH}_{2} \mathrm{CH}_{3}$ have been reported and quantum chemical calculations using active space self-consistent field (CASSCF) and coupled-cluster (CC) methods have been carried out. The computational work provides harmonic frequencies of vibrational modes of both ground and excited states. Comparison between the experimental spectra and simulation using the calculation of vibrational frequencies and FC constants allows assignment of most observed vibronic transitions. Experimentally observed transitions 
that were not predicted in the simulation can be attributed to the pJT interaction. The spectroscopic and computational results will help to scrutinize the $\mathrm{CaOCH}_{2} \mathrm{CH}_{3}$ as potential candidates for the laser-cooling of nonlinear polyatomic molecules.

\subsection{Experimental}

$\mathrm{CaOC}_{2} \mathrm{H}_{5}$ radicals were produced by $1064 \mathrm{~nm}$ laser ablation of calcium metal rod with an Nd:YAG laser (Continuum, Powerlite Precision II 8000) in the presence of ethanol under supersonic jet-cooled conditions. Our motorized stage laser ablation nozzle can drive a metal rod in a channel both rotationally and translationally has been described in previous publications. A Teflon extension was attached to the supersonic nozzle to ensure the lowest vibrational temperature. The methanol was stored in a stainless steel reservoir at room temperature and was entrained in a helium flow by passing high-pressure helium (backing pressure 180 psi above atmosphere) through the reservoir. The seeded flow was then expanded through a pinhole valve (0.3 mm diameter) into the vacuum chamber (stagnation pressure $=20$ millitirr). A $12 \mathrm{~mm}$ thick Teflon extension with a $1.5 \mathrm{~mm}$ diameter orifice at the center was attached to the supersonic nozzle for additional vibrational cooling. A dye laser (Spectra-Physics, Cobra Stretch) pumped by the second harmonic of an Nd:YAG laser (Spectra-Physics GCR-4) output was used to excite the transitions. For the LIF experiment, the laser-induced fluorescence was collected by a lens system perpendicular to both the excitation laser beam and the jet expansion, focused onto a photomultiplier tube (PMT). The spectral linewidth of the LIF

spectra is $\sim 0.1 \mathrm{~cm}^{-1}$, limited mainly by the linewidth of the pulsed dye laser $\left(\sim 0.06 \mathrm{~cm}^{-1}\right)$, 
and the residual Doppler width. The frequency of the pulsed dye laser was calibrated by a wavemeter (HighFinesse, W7). Accuracy of the vibronic transition frequencies in the LIF spectra is limited mainly by the width of the rotational contour and estimated to be $\sim 1 \mathrm{~cm}^{-1}$.

For the DF experiment, fluorescence is focused into and dispersed by a monochromator (Acton Research, SpectraPro 300i) equipped with an intensified CCD camera (Princeton Instruments, PI-MAX 512). A typical spectral resolution of $\sim 20 \mathrm{~cm}^{-1}$ was observed for DF spectra. The wavelength of the monochromator was calibrated using a mercury arc lamp. Frequency uncertainty of the DF spectra is limited by their linewidth and is estimated to be $\sim 5 \mathrm{~cm}^{-1}$.

For the maximum accuracy of the intensity determination, we used a similar technique that has been described in chapter 3. We separately used the CRDS experiment to calibrate the observed intensities in LIF (see Figure 5.6). Details about CRD can be found in chapter 3 . 


\subsection{Quantum chemical calculations}

In the present work, the ground $\left(\tilde{X}^{2} A^{\prime}\right)$ and the first two excited electronic ( $\tilde{A}_{1}$ and $\tilde{A}_{2}$ ) states of $\mathrm{CaOC}_{2} \mathrm{H}_{5}$ were calculated using the complete active space selfconsistent field (CASSCF) method with the cc-PVTZ basis set. All ab initio calculations have been performed with the Gaussian 09 program package. The $\tilde{A}_{1} \leftarrow \tilde{X}, \tilde{A}_{2} \leftarrow \tilde{X}$, and $\tilde{B} \leftarrow \tilde{X}$ electronic transitions of $\mathrm{CaOC}_{2} \mathrm{H}_{5}$ correspond to promotion of the unpaired electron in the $4 \mathrm{~s}$ orbital of $\mathrm{Ca}^{+}$to the three components of its $4 \mathrm{p}$ orbital (px; py; pz) perturbed by the presence of an ethoxy group ${ }^{98,99}$. These four orbitals (4s; 4px; 4py; 4pz) were therefore included in the active space used in the present work. In addition, the 3pz and $3 \mathrm{~d}_{\mathrm{z}}^{2}$ orbitals were included for the convergence of the CASSCF calculations because of mixing between orbitals ${ }^{3}$. In total, the active space contains 3 electrons in 6 molecular orbitals (MOs) characterized by the 3pz; $4 \mathrm{~s} ; 4 \mathrm{px} ; 4 \mathrm{py} ; 4 \mathrm{pz}$, and $3 \mathrm{~d}_{\mathrm{z}}{ }^{2}$ orbitals of calcium. Of these six MOs, the lowest-energy one (Ca 3pz) is doubly occupied in the ground electronic state, the second-lowest one (Ca 4s) is singly occupied, and the rest are virtual orbitals. Figure 5.1 illustrates the MOs used in the CASSCF calculations. Moving the d orbital out of the active space or adding more MOs does not change the calculated results significantly. Neither does use cc-PVDZ basis set instead of cc-PVTZ. Geometry optimization of the ground $(\tilde{X})$ and the $\tilde{A}_{1} / \tilde{A}_{2}$ states provided equilibrium bond lengths and bond angles that are summarized in Table 1, along with the rotational constants of both states. The $\tilde{A}_{1}$ and the $\tilde{A}_{2}$ states, originating from the $\tilde{A}^{2} E$ state of $\mathrm{CaOC}_{2} \mathrm{H}_{5}$, are nearly degenerate and strongly coupled. A state-averaged calculation with equal weights for the two states was therefore carried out for geometry optimization, which determines the minimum of the conical intersection (CI) seam. Geometric parameters of the $\tilde{A}_{1} / \tilde{A}_{2}$ 
state in Table 1 is hence for the CI. The largest change in geometry upon the $\tilde{A}_{1} / \tilde{A}_{2}-\tilde{X}$ excitation is the $\mathrm{CaO}$ bond length (30 m $\AA$ ). One, therefore, expects strong transitions to the $\mathrm{CaO}$ stretch levels. The same calculation suggests that the symmetries of the $\tilde{A}_{1}$ and the $\tilde{A}_{2}$ states are $A^{\prime}$ and $A^{\prime \prime}$, respectively. The vibrational assignment of the DF spectra in the present work (Section 5.5) is guided by quantum chemical calculations. Vibrational frequencies were calculated at the CASSCF(3,6)/cc-PVTZ level of theory. Calculation of FCFs was performed using the ezSpectrum software ${ }^{100}$. Both the harmonic frequencies of the $\tilde{X}$ state and $\tilde{A}_{1} / \tilde{A}_{2}$-state vibrational frequencies listed in Table 2. Gaussian lineshape with full-width at half-maximum (FWHM) of $5 \mathrm{~cm}^{-1}$ was used to generate the calculated spectrum of $\tilde{A}_{1}^{2} A^{\prime} / \tilde{A}_{2}^{2} A^{\prime \prime} \leftarrow \tilde{X}^{2} A^{\prime}$ and $30 \mathrm{~cm}^{-1}$ was used to generate the calculated spectrum of the $\tilde{A}_{1}^{2} A^{\prime} / \tilde{A}_{2}^{2} A^{\prime \prime} \rightarrow \tilde{X}^{2} A^{\prime}$ transition in Figure 5.2 and 5.3 respectively. The energy separation between the minimum of the $\sim \mathrm{X}$ state and the CI of the $\tilde{A}_{1}$ and the $\tilde{A}_{2}$ states (without zero-point-energy corrections) was found to be 15,390 $\mathrm{cm}^{-1}$, which shall be compared to the experimentally observed $\tilde{A}_{1}^{2} A^{\prime} \leftarrow \tilde{X}^{2} A^{\prime}$ and the $\tilde{A}_{2}^{2} A^{\prime \prime} \leftarrow \tilde{X}^{2} A^{\prime}$ origin band transition frequencies: 15,850 and $15,913 \mathrm{~cm}^{-1}$, respectively. The relatively small discrepancy between the experimental and calculated excitation energies suggests that dynamic electron correlation corrections to CASSCF calculations of the ground and the excited electronic states cancel to a large extent. We also performed CCSD calculation similar to CaOCH3 and detailed can be found elsewhere ${ }^{101}$. 


\subsection{Results}

\subsubsection{LIF spectrum}

The experimental LIF spectrum of the $\tilde{A} \leftarrow \tilde{X}$ transition of $\mathrm{CaOC}_{2} \mathrm{H}_{5}$ features six doublets with a frequency interval of about $67 \mathrm{~cm}^{-1}$ between each pair of peaks. The left and right peaks of each pair represent $\widetilde{A_{1}^{2}} \hat{A} \leftarrow \tilde{X}^{2} \dot{A}$ and $\widetilde{A_{2}^{2}} \dot{A} \leftarrow \tilde{X}^{2} \dot{A}$ transition respectively (see Figure 5.2).

The strongest doublet centered at $15882 \mathrm{~cm}^{-1}$ is assigned to the origin transition. Three pair of doublets centered at 390,532 and $1170 \mathrm{~cm}^{-1}$ to the blue of the origin band can be assigned to transition to the $\hat{v}=1$ levels of the $\mathrm{CaO}$ stretch mode $\left(12_{0}^{1}\right)$, the OCC bending mode $\left(11_{0}^{1}\right)$ and the CO stretch mode $\left(8_{0}^{1}\right)$, respectively.

Two pairs of weaker doublets observed with centered of $93 \mathrm{~cm}^{-1}$ and $130 \mathrm{~cm}^{-1}$ blue of the origin band is not predicted by either CCSD or CASSCF simulation. The most reasonable assignment of this doublet is the transition to the $\dot{v}=1$ level of the CaOC bending mode in plane $\left(13_{0}^{1}\right)$ and out of the plane $\left(21_{0}^{1}\right)$ for which the CASSCF and CCSD calculations predict a harmonic frequency of $93 \mathrm{~cm}^{-1}$ and $140 \mathrm{~cm}^{-1}$ respectively. The doublet results from the splitting of the vibrational transition into two SO levels like the other lines in the spectrum. Due to the vibrational symmetry, transitions from vibrationless ground level of the $\tilde{X}^{2} A$ to the $13^{1}$ level of the $\widetilde{A_{1}^{2}} \hat{A}$ state and $21^{1}$ level of the $\widetilde{A_{2}^{2}} \dot{A}$ state are forbidden. However, such transitions often gain intensity through vibronic interactions. 
As stated in section 2, the origin transitions are power-saturated therefore, jet-cooled pulsed-laser CRD spectroscopy measurements have been undertaken for better determination of the VBRs of the origin transitions. LIF and CRD spectra of the origin, $12_{0}^{1}$ and $11_{0}^{1}$ are compared in figure 5.3. The determined saturation factor for the origin transitions in the LIF experiment is 2.75. Therefore, FC factors for non-origin transitions determined from the LIF spectrum are reduced by a factor of 2.75 relatives to the origin transitions followed by normalization. The saturation-free FC factors are listed in Table 3. The FC factor, i.e., VBR of the origin transitions so determined $(f=.841)$ is estimated to have a relative error of $10 \%$ (determined from the error of 3 repeated experimental trace) mainly due to the fluctuation of free radical concentration in the jet expansion and the issue involving the CRD calibration to avoid effects of saturation. 


\subsubsection{DF spectra obtained by pumping the origin transitions}

Figure 5.3c and 5.3e illustrate the DF spectra obtained by pumping the $\tilde{A}_{1} \leftarrow \tilde{X}$ and $\tilde{A}_{2} \leftarrow \tilde{X}$ origin bands, respectively ${ }^{3}$. Because these two spectra have the same lower electronic state, the same strong transitions are present in both of them. In both spectra, close to the zero-redshift peak (the $\tilde{A}_{1} \rightarrow \tilde{X}$ or $\tilde{A}_{2} \rightarrow \tilde{X}$ origin band), there is a peak shifted by $\sim 65 \mathrm{~cm}^{-1}$ with respect to the origin band. In the $\tilde{A}_{1} \rightarrow \tilde{X}$ spectrum it is blue-shifted, while it is redshifted in the $\tilde{A}_{2} \rightarrow \tilde{X}$ spectrum. In addition, several other weaker peaks are observable in the DF spectra either blue-shifted (in the $\tilde{A}_{1} \rightarrow \tilde{X}$ spectrum) or redshifted (in the $\tilde{A}_{2} \rightarrow \tilde{X}$ spectrum) by $\sim 65 \mathrm{~cm}^{-1}$ from stronger peaks. The interval of $\sim 65 \mathrm{~cm}^{-1}$ corresponds to the energy separation between the $\sim \mathrm{A}_{1}$ and $\sim \mathrm{A}_{2}$ zeropoint levels determined in the LIF spectrum. The weaker "satellite" peaks, asterisked in Figure. 5.3c and 3e are therefore attributed to collisional induced population transfer between the $\tilde{A}_{1}$ and the $\tilde{A}_{2}$ states. Previously, Bernath and coworkers ${ }^{2}$ reported relaxation from the $\tilde{A}_{2}$ state to the $\tilde{A}_{1}$ state after laser excitation to the $\tilde{A}_{2}$ state of $\mathrm{CaOC}_{2} \mathrm{H}_{5}$ and other similar alkaline earth monoalkoxide radicals produced in a Broida oven. In that work, $\tilde{A}_{1} \rightarrow \tilde{X}$ and $\tilde{A}_{2} \rightarrow \tilde{X}$ transitions possess similar intensities in the DF spectra. In the present DF spectra of the jet-cooled $\mathrm{CaOC}_{2} \mathrm{H}_{5}$, peaks due to population transfer are much weaker than their "parent” peaks, indicating much less efficient relaxation or redistribution of excited-state population. Almost all strong peaks in the DF spectra are reproduced in the simulated spectrum and can be assigned on the basis of the calculated 
vibrational frequencies and FCFs. The peaks at $386 \mathrm{~cm}^{-1}$ and $514 \mathrm{~cm}^{-1}$ were observed in the previous work by Bernath et al. ${ }^{2}$ using a Broida oven. The center frequencies are determined in the present jet-cooled experiment with higher precision thanks to the better resolution. In Ref. ${ }^{2}$ these two peaks were assigned to transitions to the $\mathrm{CaO}$ stretch $\left(v_{12}\right)$ and OCC bending $\left(v_{11}\right)$ modes, respectively. The present CASSCF calculation predicts these two vibrational modes to have vibrational frequencies of 367 and $514 \mathrm{~cm}^{-1}$ and large FCFs (Figure 5.3a), which confirms the previous assignments. Two relatively weaker peaks were observed at 916 and $1170 \mathrm{~cm}^{-1}$ in the present jet-cooled experiment. CASSCF calculation predicts two transitions with considerable intensities at 974 and $1294 \mathrm{~cm}^{-1}$. The former transition is due to CC stretch $\left(v_{10}\right)$ while the latter due to CO stretch $\left(v_{8}\right)$, both of which are in conjunction with $\mathrm{CaO}$ bond stretch. The peaks at 916 and $1170 \mathrm{~cm}^{-1}$ are therefore assigned to the CC stretch and the CO stretch, respectively. In the $\tilde{A}_{1} \rightarrow \tilde{X}$ spectrum, one peak is observable at $93 \mathrm{~cm}^{-1}$ that is absent in the $\tilde{A}_{2} \rightarrow \tilde{X}$ spectrum. This difference can be explained by the fact that the $\tilde{A}_{1}$ and $\tilde{A}$ states have different symmetries ( $A^{\prime}$ and $A^{\prime \prime}$ ) and are coupled by the pJT effect, which distorts the $\mathrm{PESs}^{96,97}$. Although the $A^{\prime}$ state PES retains its $\mathrm{C}_{\mathrm{s}}$ symmetry, the $A^{\prime \prime}$ state PES is reduced to $\mathrm{C}_{1}$ at its minima. The displacement is along coordinates of the pJT modes. Such distortion of PESs and the lowering of symmetry result in different FCFs for transitions from the zero-energy levels of the $A^{\prime}$ and the $A^{\prime \prime}$ states to the ground-state vibrational levels of the pJT modes. Frequency calculation at the CASSCF(3,6)/cc-PVTZ level of theory shows that both the symmetric $\left(a^{\prime}\right)$ and the anti-symmetric $\left(a^{\prime \prime}\right)$ pJT modes are Ca-O-C bending, with the $a^{\prime}$ mode in the Cs plane and the $a^{\prime \prime}$ mode out of the plane. The predicted harmonic frequencies of the $a^{\prime}\left(v_{13}\right)$ and the $a^{\prime \prime}\left(v_{21}\right)$ pJT 
modes of the ground state are 96 and $140 \mathrm{~cm}^{-1}$, respectively. The observed peak at $93 \mathrm{~cm}^{-}$ ${ }^{1}$ is therefore assigned to the in-plane Ca-O-C bending mode. The FCF calculation predicts that the first overtone of this mode has stronger intensity than the fundamental (Figure 5.3a). The discrepancy between the experimental observation and the prediction may be explained again by the distortion of $\tilde{A}_{1} / \tilde{A}_{2}$-state PESs. Because of the vibronic coupling, transition intensities of pJT active modes are no longer determined only by their symmetries and FCFs. Instead, vibronic symmetries need to be taken into account. Transition to symmetric ( $a^{\prime}$ ) pJT mode was observed only in the $\tilde{A}_{1} \rightarrow \tilde{X}$ spectrum, but not the $\tilde{A}_{2} \rightarrow \tilde{X}$ spectrum. Such observation suggests that the $\tilde{A}_{1}$ state is of $A^{\prime}$ symmetry, while the $\tilde{A}_{2}$ state is of $A^{\prime \prime}$ symmetry, which is consistent with the CASSCF calculations (Section 3).

In the present work, we are reporting mainly the VBRs and FC factors. In addition to CASSCF we also reports the CCSD simulation ( $3 \mathrm{~b}$ and 3d) in the present work. As stated in 2, the experimental determination of the origin-transition FC factor from the DF spectra is difficult due to the contamination of the scattering of the excitation laser. It is therefore fixed to the value determined in the LIF and CRD experiment (.841). FC factors of the other transitions are determined by the maintenance of their experimentally determined intensity ratios while keeping the FC factors normalized. 


\subsubsection{DF spectra obtained by pumping other vibronic bands}

DF spectra obtained by pumping strong vibronic bands in the LIF spectrum, namely, $13_{0}^{1}, 21_{0}^{1}, 12_{0}^{1}, 11_{0}^{1}, 8_{0}^{1}$, are represented in 5b-f. Compared to those obtained by pumping the origin band (5a), DF transition from a vibronic level of the $\tilde{A}$ state to those $\tilde{X}$-state vibrational levels of the pumped mode gain intensity. Additional because of the Duschinsky mixing transitions to combination levels of the pumped mode and other modes may also gain intensities.

A simple technique that reveals this relation is to blue-shift DF spectra by the ground-state frequencies of the pumped modes. The resulting spectra are shown in Figure 5.4 and 5.5. As demonstrated, there are both $\mathrm{CaO}$ stretch $\left(v_{12}\right)$ and (p)JT -active CaOC bending $\left(v_{13}\right)$ in-plane modes are mixed with the OCC bending $\left(v_{11}\right)$ mode, which can be explained by the elongation of the $\mathrm{CaO}$ bond length upon the electronic excitation, and hence the large mixing between the $\mathrm{CaO}$ stretch and other modes.

When the $11_{0}^{1}$ (OCC bending) band in the LIF spectrum is pumped, the DF spectrum contained, among others, a strong peak at $1130 \mathrm{~cm}-1$ for $V_{00}+502$ and 1160 for $V_{00}+568$. They are assigned as "Y" and "Z" respectively. These two transitions are not line up with strong transition around $1170 \mathrm{~cm}^{-1}$. One possible reason of the shifting is probably fermi-resonance. But as we do not have any experimental evidence or theoretical support, that's why we assigned them as unanalyzed transitions. Another peak in the spectrum obtained by pumping $13_{0}^{1}$ labeled ' $\mathrm{Y}$ ', in $5 \mathrm{~b}$ cannot assigned either can be attributed to impurity. 


\subsection{Implications to direct laser cooling of $\mathrm{CaOC}_{2} \mathrm{H}_{5}$ and TRSV}

\section{measurements}

We now discuss the implication of the determined FC factors to direct laser cooling of the $\mathrm{CaOC}_{2} \mathrm{H}_{5}$ radical. In such an experiment, pre-cooled molecules are excited to the $\widetilde{A_{1}}$ state. Besides the origin transitions, spontaneous emission to both the $12_{1}$ level and even the $11_{1}$ level is significant. If a cooling laser is used to excite the origin band and two re-pumping lasers are employed to return the population from the $4_{1}$ and $3_{1}$ levels to the cooling cycle, the sum of VBRs for spontaneous emission transitions to $\tilde{X}^{2} A_{1}$-state vibrational levels other than $0_{0}, 11_{1}$ and $12_{1}$ is 0.083 , which implies that a molecule will experience on average $\sim 20$ scattering events before it decays to vibrational “dark” states that are not addressed by the re-pumping lasers.

Measurement of energy level structure of fundamental and overtone levels of the Ca-O-C bending mode ( $v_{13}$ and $\left.v_{21}\right)$ and transitions involving them is a prerequisite of the proposed investigation of TRSV interactions and the detection of eEDM. The $v^{\prime \prime}{ }_{13}=1$ state is the proposed eEDM measurement state. Population transfer from the ground vibrational level of the $\tilde{X}^{2} A_{1}$ state to the $v^{\prime \prime}{ }_{13}=1$ state can be achieved using stimulated Raman adiabatic passage (STIRAP)39-42 using $v^{\prime}{ }_{13}=1$ as the intermediate state. 


\subsection{Conclusion}

We report vibrationally resolved LIF and DF spectra of the $\tilde{A}_{1} / \tilde{A}_{2} \leftarrow \tilde{X}$ transition of the calcium methoxide radical. Vibrational assignment has been made on the basis of CASSCF- and CC-calculated vibrational frequencies and FC factors for the allowed vibronic transitions. It has been found that both $a b$ initio methods predict the vibrational frequencies to a significant degree of accuracy. These calculations also do a reasonably good job for the intensity of the allowed, strong transitions. However other forbidden transitions are weakly observed in the spectrum which is not predicted to have intensity by either calculation. In the present work, relative intensities were determined in LIF and DF measurements, but our measurements have two limitations. First, the fluorescence signal is contaminated by the excitation laser scattering. Second, the excitation transitions of the origin band are power saturated. The second issue could be largely avoided by using continuous-wave (cw) excitation laser sources.

A separate cavity-ring down experiment has been performed to compensate these two issues by making use of the jet-cooled pulsed-laser CRD spectra. The signal-to-noise ratio of the current CRD measurement is somewhat lower than the LIF experiment. It can be significantly improved if cw laser sources are used. 


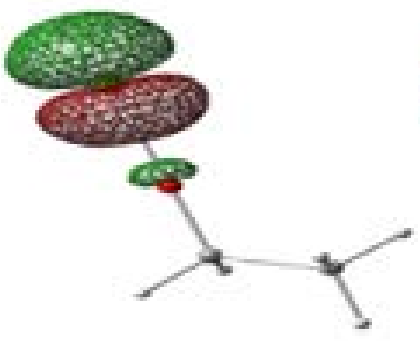

$\mathrm{Ca} 3 p_{1}$

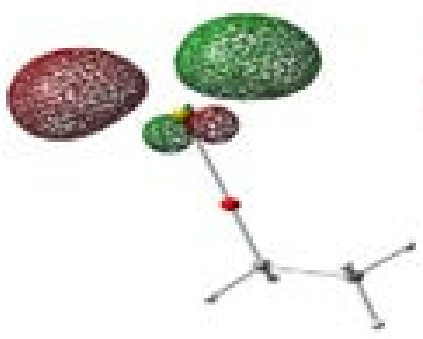

$\mathrm{Ca} 4 p_{x}$

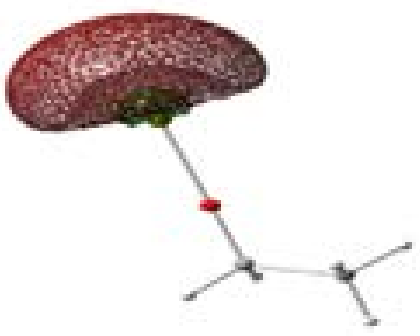

$\mathrm{Ca} 45$

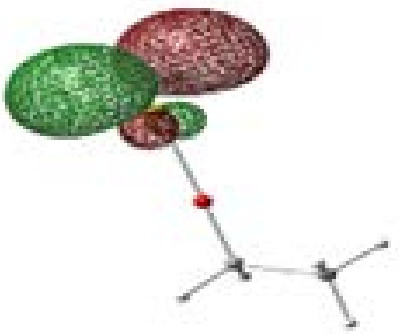

$\mathrm{Ca} 4 p_{y}$

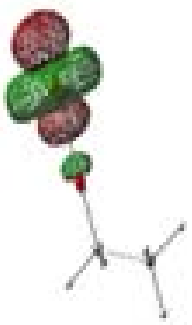

$\mathrm{Ca} 3 d_{2} 2$

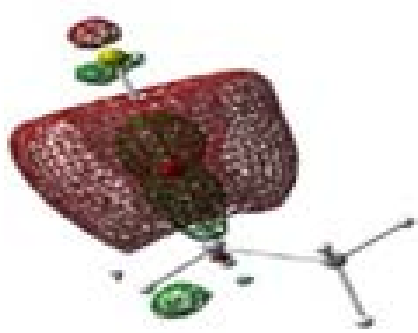

$\mathrm{Ca} 4 p_{x}$

Figure 5.1: The molecular orbitals of $\mathrm{CaOCH}_{2} \mathrm{H}_{3}$ included in CASSCF calculation 
(d)

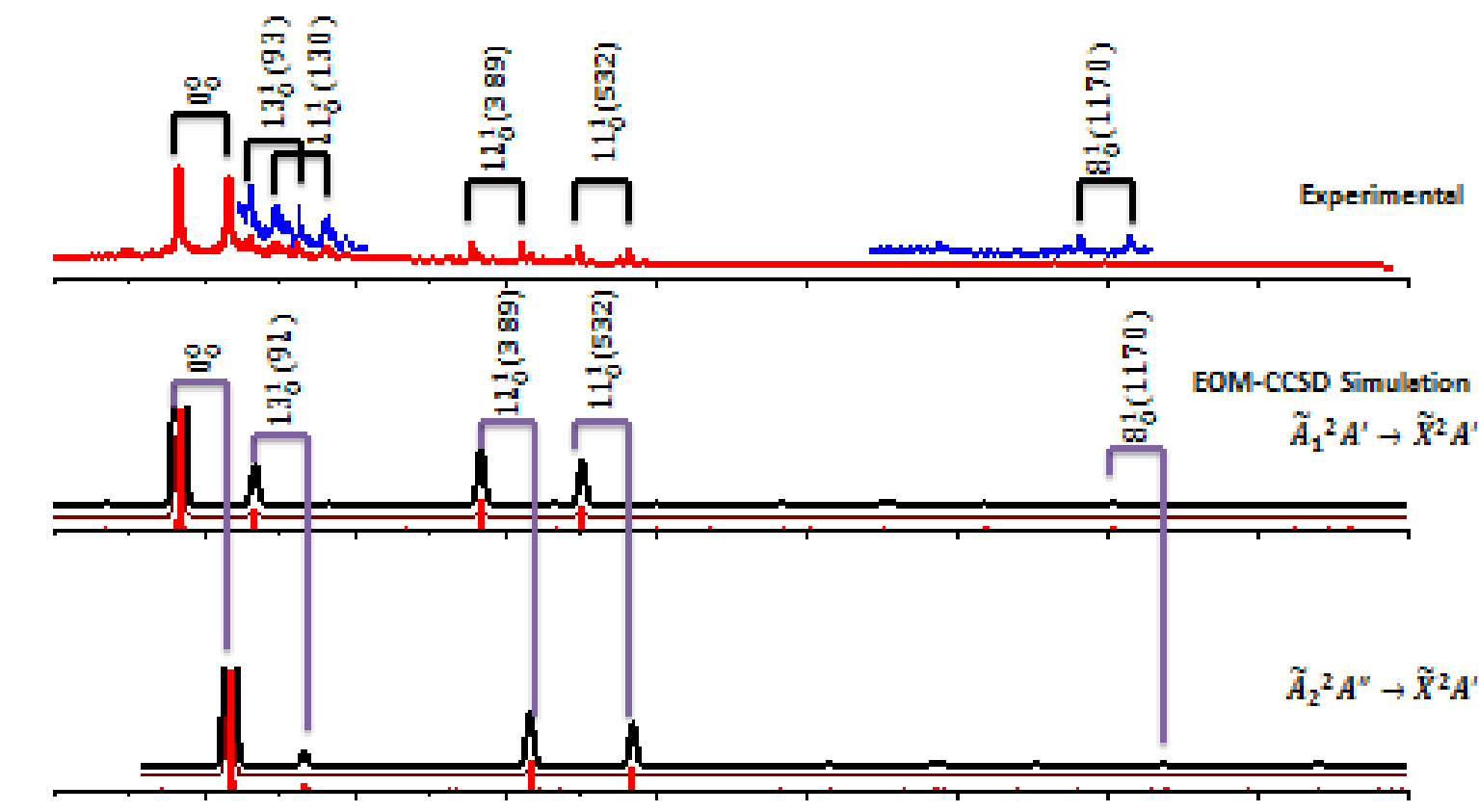

(a)

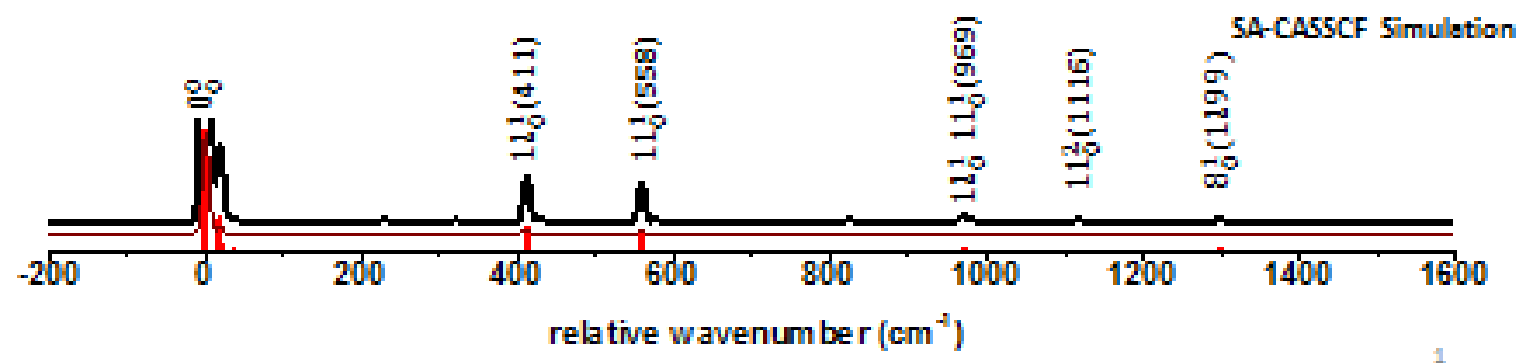

Figure 5.2: (a) Simulated by state averaged CASSCF method (b and c) Simulated for the $\tilde{A}_{1}^{2} A^{\prime} \leftarrow \tilde{X}^{2} A^{\prime}$ and $\tilde{A}_{2}^{2} A^{\prime \prime} \leftarrow \tilde{X}^{2} A^{\prime}$ transition of Ca-ethoxide. Wavenumbers are relative to the origin band at $15882 \mathrm{~cm}^{-1}$. Numbers in parentheses are relative wavenumbers in $\mathrm{cm}^{-1}$. 

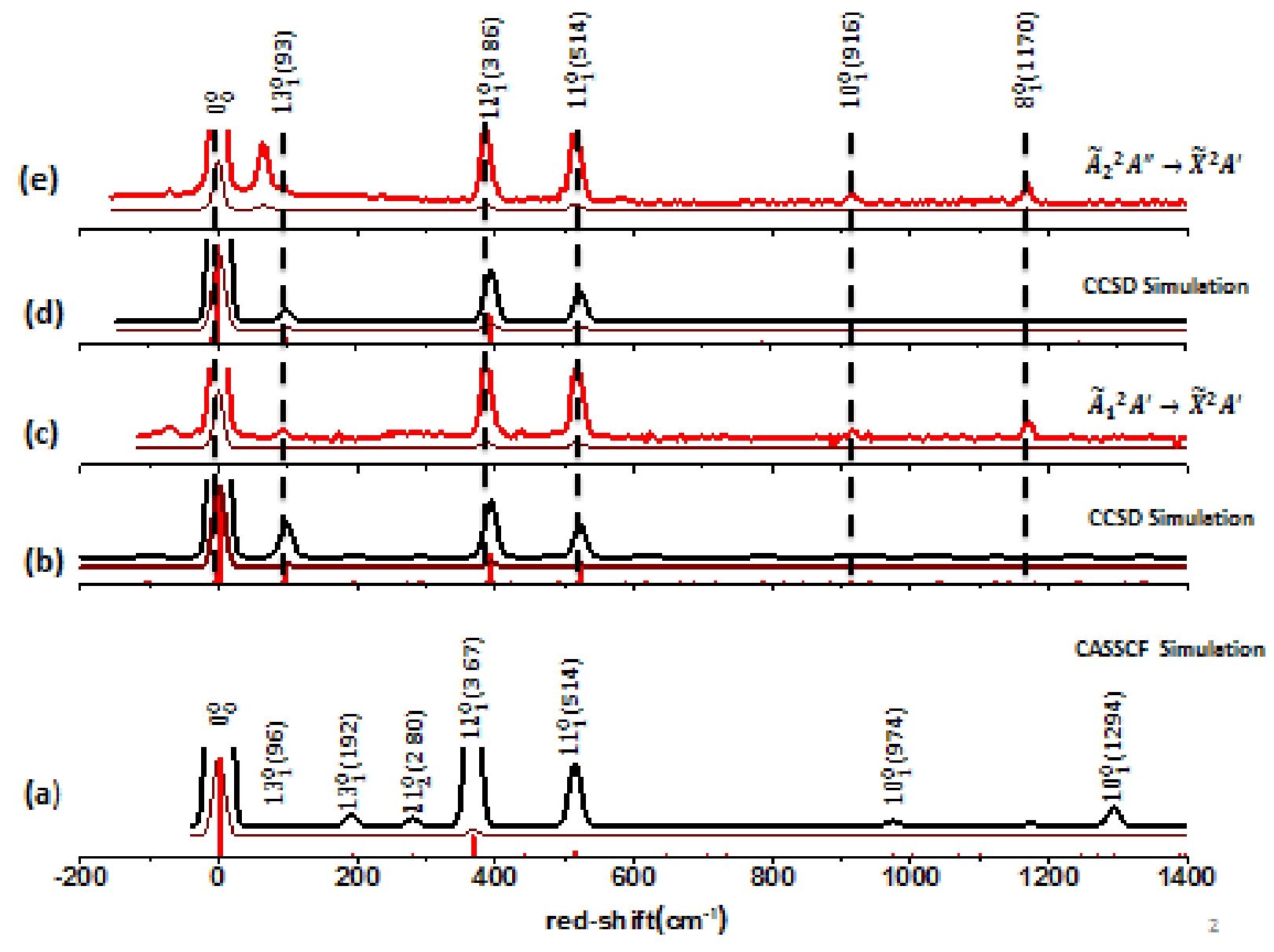

Figure 5.3: (a) simulated by state average CASSCF method. (b and d) simulated for the $\tilde{A}_{1}^{2} A^{\prime} \rightarrow \tilde{X}^{2} A^{\prime}$ and $\tilde{A}_{2}^{2} A^{\prime \prime} \rightarrow \tilde{X}^{2} A^{\prime}$ transition of Ca-ethoxide. DF spectra by pumping the (c) $\tilde{A}_{1}^{2} A^{\prime} \leftarrow \tilde{X}^{2} A^{\prime}$ and (e) $\tilde{A}_{2}^{2} A^{\prime \prime} \leftarrow \tilde{X}^{2} A^{\prime}$ origin transitions. Numbers in the parentheses are red-shift in $\mathrm{cm}^{-1}$. The asterisked peaks in (d) are due to collision-induced population transfer. 


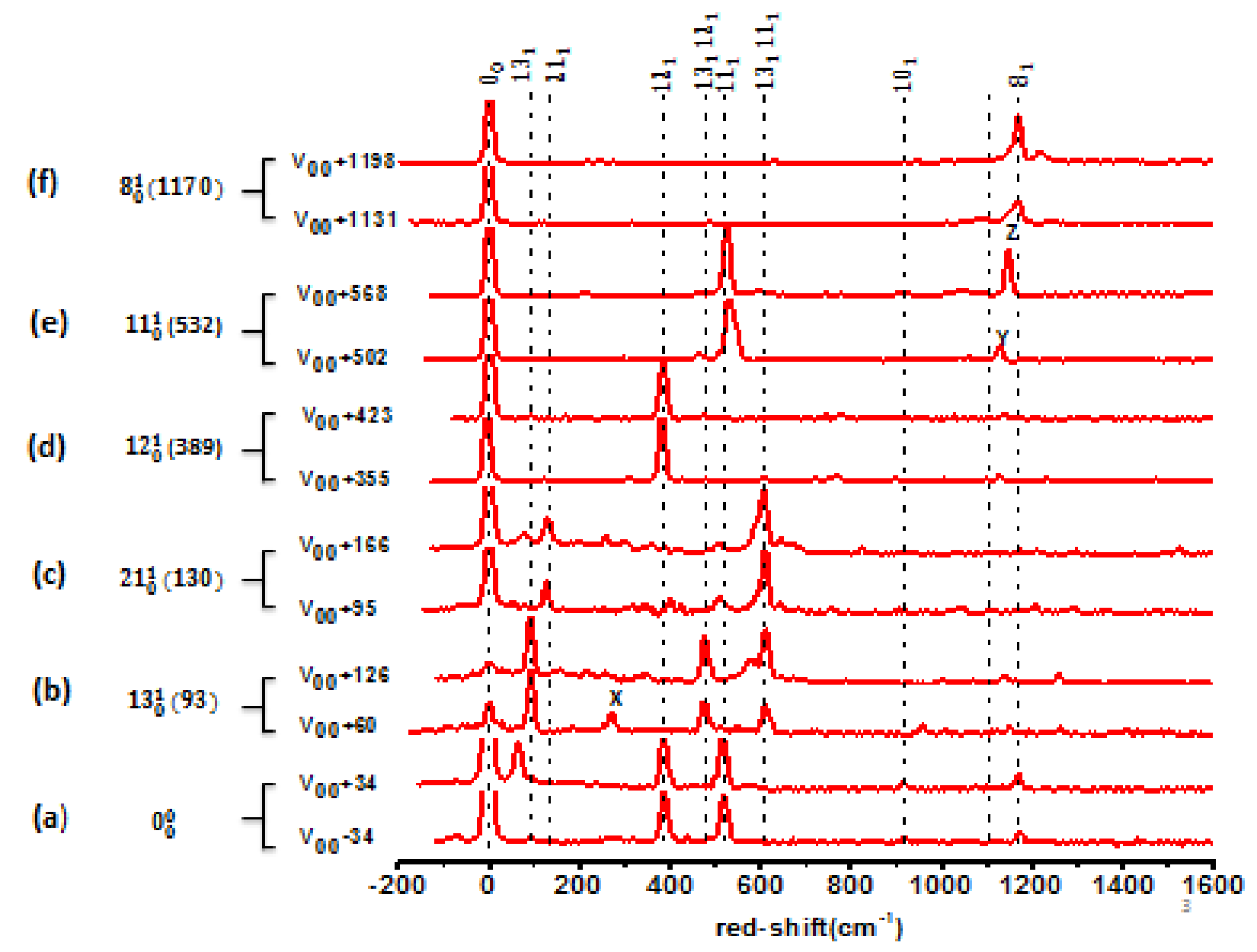

Figure 5.4: DF spectra by pumping different vibronic bands of the $\tilde{A}_{1} / \tilde{A}_{2} \leftarrow \tilde{X}$ transitions of ca-ethoxide. Numbers in the parentheses are red-shift in $\mathrm{cm}^{-1}$. "X", "Y" and “Z” denotes unidentified transitions. 


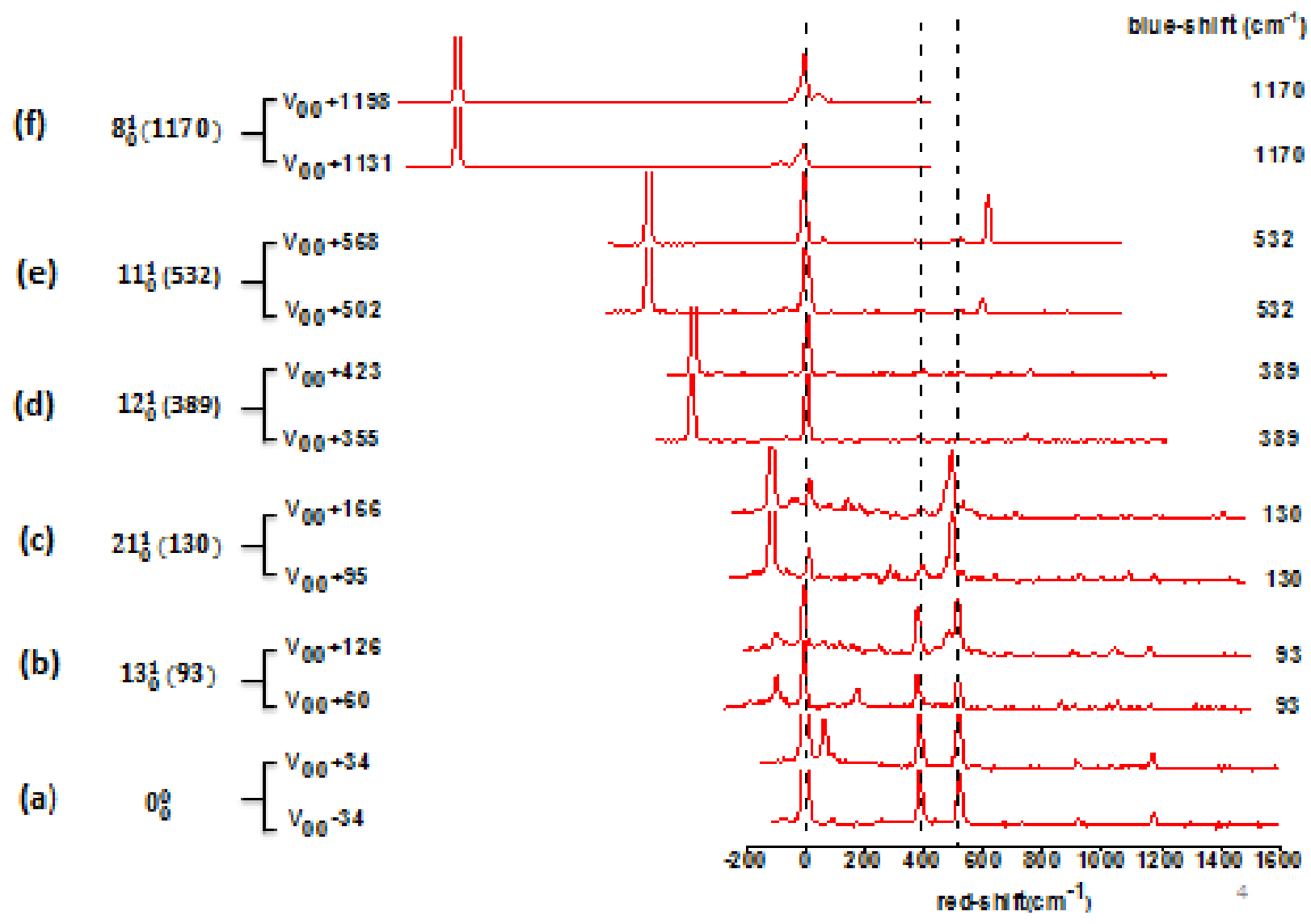

Figure 5.5: DF spectra by pumping different vibronic bands of the $\tilde{A}_{1} / \tilde{A}_{2} \leftarrow \tilde{X}$ transitions of ca-ethoxide. The numbers in the parentheses are red-shift in $\mathrm{cm}^{-1}$. Spectra of bands other than origin band in the LIF spectrum are blue-shifted. 


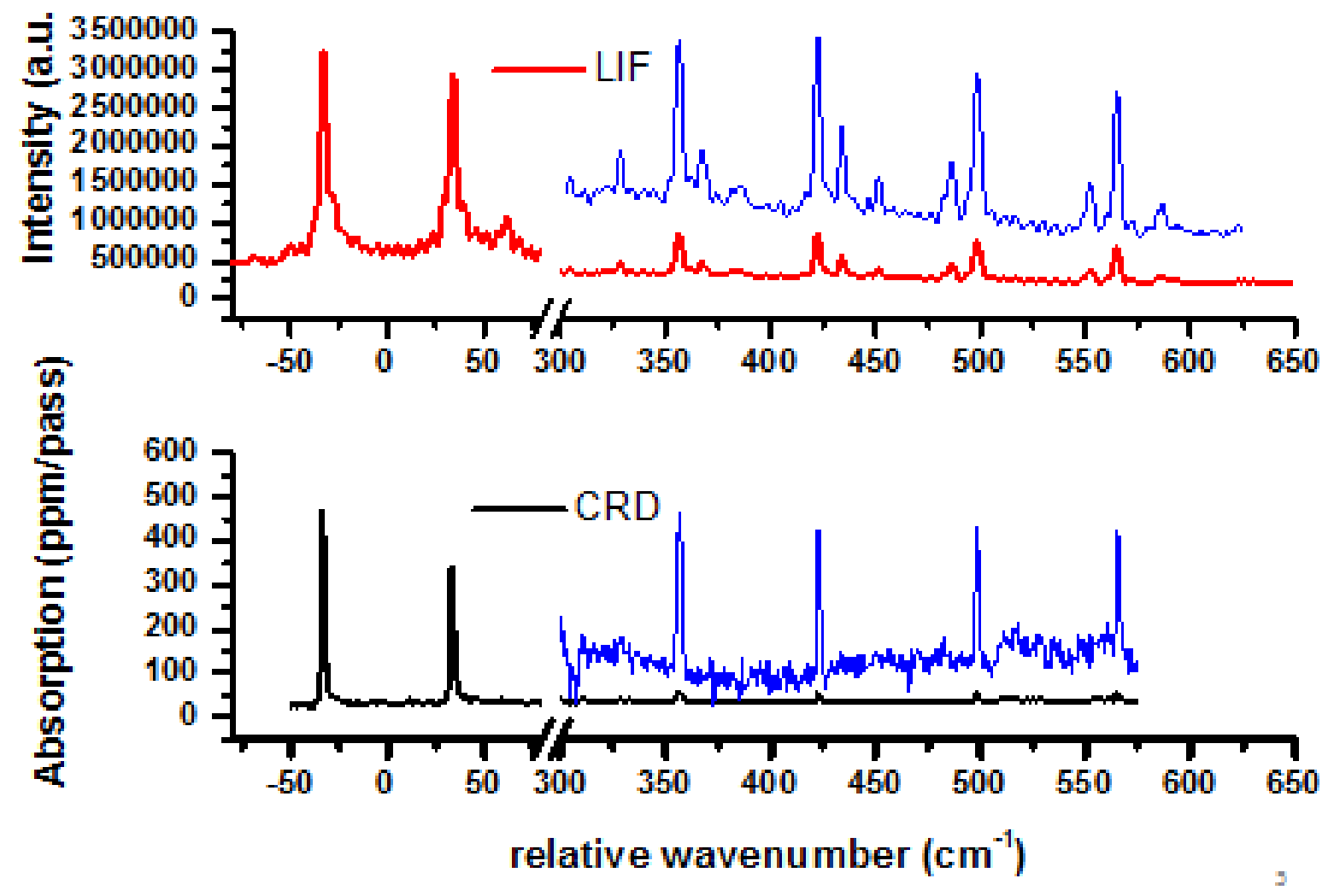

Figure 5.6: Comparison of (a) LIF and (b) CRD spectra of the origin and band transitions of $\mathrm{CaOCH}_{2} \mathrm{CH}_{3}$ that shows the power saturation of the origin transitions in the LIF experiment 
Table 5.1: Geometric parameters, rotational constants, and $\tilde{A}-\tilde{X}$ excitation energy of the $\mathrm{CaOCH}_{2} \mathrm{CH}_{3}$ radicals.

\begin{tabular}{|c|c|c|c|c|}
\hline & \multicolumn{2}{|c|}{ CASSCF(3,6)/cc-PVTZ } & \multicolumn{2}{c|}{ CCSD/EOM-CCSD/cc-PVDZ } \\
\hline & $\tilde{X}$ state & $\tilde{A}_{1} / \tilde{A}_{2}$ state & $\tilde{X}$ state & $\tilde{A}_{1} / \tilde{A}_{2}$ state \\
\hline $\mathrm{rCaO}(\AA)$ & 2.014 & 1.984 & 2.115 & 2.087 \\
\hline $\mathrm{rCO}(\AA)$ & 1.372 & 1.375 & 1.417 & 1.424 \\
\hline $\mathrm{rCC}(\AA)$ & 1.523 & 1.521 & 1.532 & 1.530 \\
\hline$\angle \mathrm{CaOC}(\mathrm{deg})$. & 179 & 179 & 179 & 179 \\
\hline$\angle \mathrm{OCC}(\mathrm{deg})$. & 112 & 112 & 112 & 112 \\
\hline $\mathrm{A}\left(\mathrm{cm}^{-1}\right)$ & 0.816 & 0.821 & .778 & .773 \\
\hline $\mathrm{B}\left(\mathrm{cm}^{-1}\right)$ & 0.066 & 0.067 & .062 & .063 \\
\hline $\mathrm{C}\left(\mathrm{cm}^{-1}\right)$ & 0.063 & 0.064 & .059 & .059 \\
\hline$\Delta E^{\tilde{A}_{1} / \tilde{A}_{2} \leftarrow \tilde{X}}$ \\
$\left(\mathrm{~cm}^{-1}\right)^{*}$ & \multicolumn{2}{|r|}{$15390^{\mathrm{a}}$} & & $15869^{\mathrm{a}}$ \\
\hline
\end{tabular}


Table 5.2: Harmonic vibrational frequencies of $\mathrm{CaOCH}_{2} \mathrm{CH}_{3}$ in the $\tilde{X}^{2} A_{1}$ and $\tilde{A}^{2} E$ states (in $\mathrm{cm}^{1}$ ). Vibrational modes are numbered following the Herzberg convention.

\begin{tabular}{|l|l|l|l|l|l|l|l|l|}
\hline mode & sym. & \multicolumn{3}{|l|}{-state freq. } & \multicolumn{3}{l|}{$\widetilde{A_{1}} / \widetilde{A_{2}}$ state freq. } & description \\
\hline & & Calc. 1 & Calc. 2 & Expt. & Calc. 1 & Calc.2 & Expt. & \\
\hline$v_{1}$ & $a^{\prime}$ & 3198 & 3132 & & 3202 & 3136 & & \\
\hline$v_{2}$ & $a^{\prime}$ & 3141 & 3049 & & 3145 & 3052 & & \\
\hline$v_{3}$ & $a^{\prime}$ & 3080 & 2992 & & 3096 & 3010 & & \\
\hline$v_{4}$ & $a^{\prime}$ & 1658 & 1522 & & 1658 & 1520 & & \\
\hline$v_{5}$ & $a^{\prime}$ & 1610 & 1494 & & 1609 & 1495 & & \\
\hline$v_{6}$ & $a^{\prime}$ & 1557 & 1427 & & 1558 & 1426 & & \\
\hline$v_{7}$ & $a^{\prime}$ & 1514 & 1393 & & 1517 & 1393 & & \\
\hline$v_{8}$ & $a^{\prime}$ & 1294 & 1216 & 1170 & 1299 & 1210 & 1170 & CO stretch \\
\hline$v_{9}$ & $a^{\prime}$ & 1174 & 1108 & & 1178 & 1109 & & \\
\hline$v_{10}$ & $a^{\prime}$ & 974 & 927 & 916 & 983 & 930 & & CC stretch \\
\hline$v_{11}$ & $a^{\prime}$ & 514 & 527 & 514 & 558 & 528 & 532 & OCC bending \\
\hline$v_{12}$ & $a^{\prime}$ & 367 & 391 & 386 & 411 & 391 & 389 & CaO stretch \\
\hline$v_{13}$ & $a^{\prime}$ & 96 & 90 & 93 & 114 & 90 & 93 & CaOC bending \\
\hline$v_{14}$ & $a^{\prime \prime}$ & 3201 & 3140 & & 3206 & 3141 & & \\
\hline$v_{15}$ & $a^{\prime \prime}$ & 3084 & 3022 & & 3104 & 3045 & & \\
\hline$v_{16}$ & $a^{\prime \prime}$ & 1597 & 1481 & & 1598 & 1480 & & \\
\hline$v_{17}$ & $a^{\prime \prime}$ & 1431 & 1308 & & 1437 & 1304 & & \\
\hline$v_{18}$ & $a^{\prime \prime}$ & 1289 & 1182 & & 1291 & 1177 & & \\
\hline$v_{19}$ & $a^{\prime \prime}$ & 861 & 805 & & 863 & 806 & & \\
\hline$v_{20}$ & $a^{\prime \prime}$ & 302 & 293 & & 303 & 288 & & \\
\hline$v_{21}$ & $a^{\prime \prime}$ & 140 & 122 & & 161 & 118 & 130 & CaOC bending \\
\hline & & & & & & & & \\
\hline
\end{tabular}




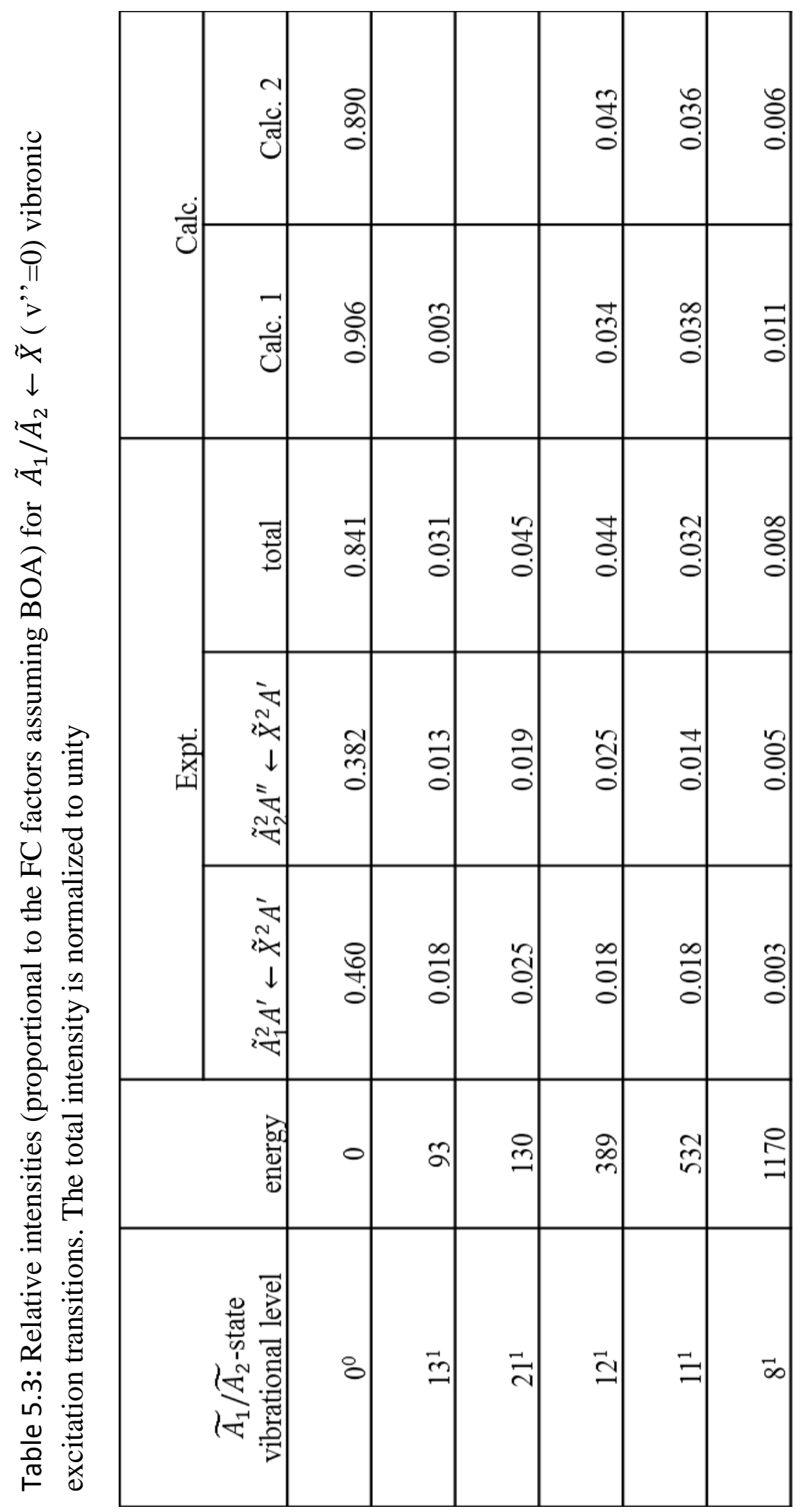




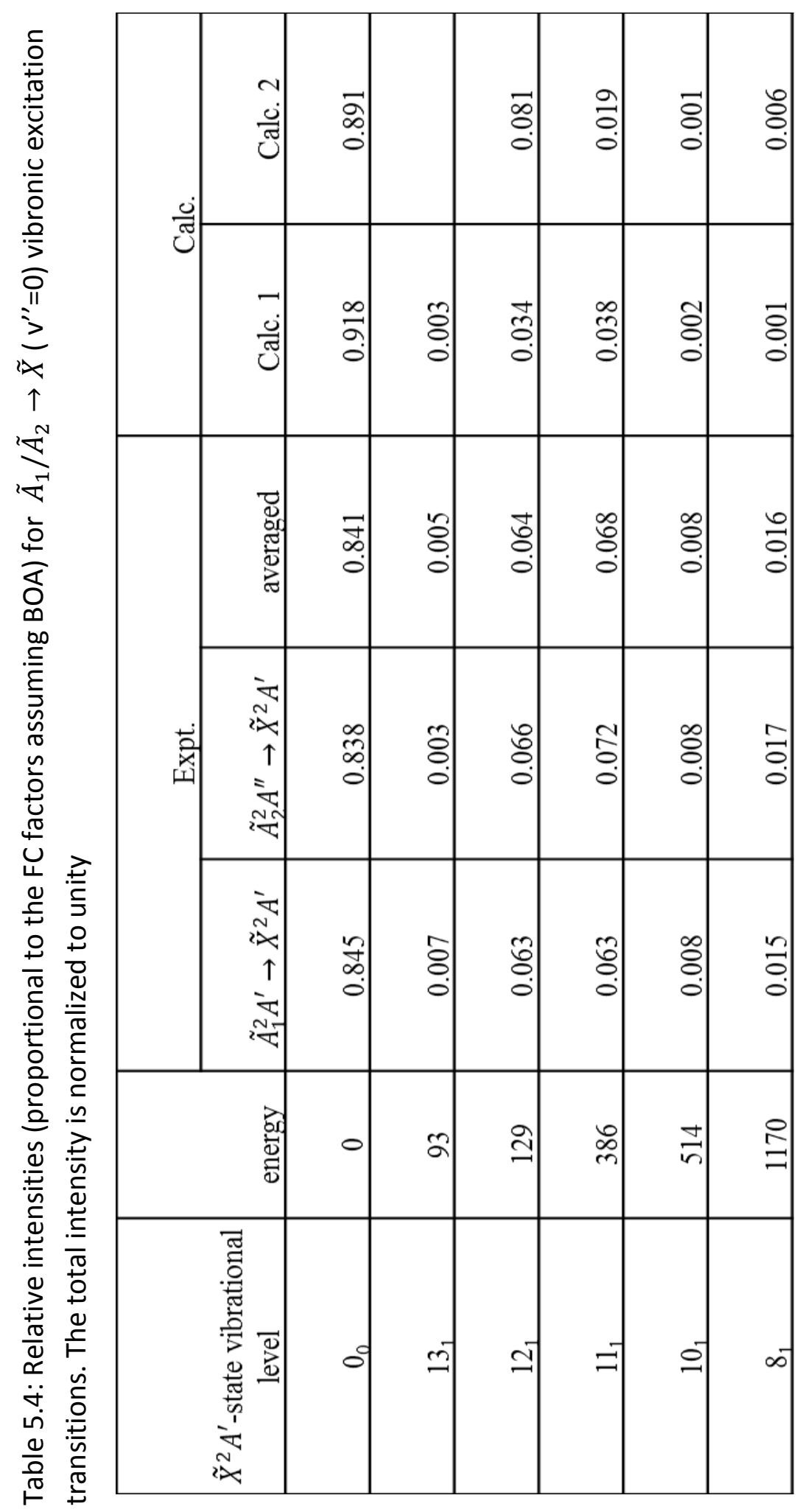




\section{CHAPTER VI}

\section{LASER-INDUCED FLUORESCENCE AND DISPERSED- FLUORESCENCE SPECTROSCOPY OF Ca-ISOPROPOXIDE}

The complicated excited-state structure of metal-containing molecules achieved great interest in theoretical molecular spectroscopy. The importance of studying these molecules has been described in chapter 1 . We also described the importance of studying these molecules as in fundamental physics as recently calcium metal-containing alkoxide radicals have been proposed as a candidate molecules for laser cooling process ${ }^{4}$. The conical intersection in the excited state, where two spin-orbit component overlap makes the theoretical study of these molecules more difficult.

An effective approach to study the PJT interaction is by asymmetric isotope or methyl substitution of JT molecules. The asymmetric isotope substitution, such as deuteration, lowers the vibrational symmetry due to mass effect but the electronic symmetry, i.e., the symmetry of the PESs, remains unchanged. Asymmetrically deuterated isotopologues, therefore, work as a "bridge" between the JT effect and the PJT effect. Asymmetric methyl substitution, which lowers the molecular symmetry from $C_{3 \mathrm{v}}$ to $C_{\mathrm{s}}$, can, therefore, be used to induce PJT effect. In chapter, we have described the effect 
of asymmetric methyl-substitution. The $\tilde{A}^{2} \mathrm{E}$ state split into two near-degenerate states, one with $A^{\prime}$ and other with $A^{\prime \prime}$ symmetry. The near degeneracy of these two states results in strong vibronic interaction (PJT effect) and the formation of a CI. Further substitution of a methyl group to ca-ethoxide lowered the molecular symmetry from $C_{s}$ to $C_{1}$. In this chapter, the harmonic frequency assignment of the molecular vibration of Caisopropoxide by laser-induced fluorescence and dispersed-fluorescence will be illustrated. The experimental condition was used similar to ca-methoxide and caethoxide.

${ }^{102}$ For the calibration of the saturation factor we performed cavity ring-down spectroscopy measurement and essentially the experimental conditions were similar except for the radical generation we used propanol in presence of calcium metal vapor and carrier gas as Helium.

As we already noticed from the study of ca-methoxide and ca-isopropoxide the couple-cluster (CCSD) method predicts the vibrational frequency and intensities much accurate the complete-active space self-consistent (CASSCF) method, so for the harmonic frequency assignment we used only CCSD method for Ca-isopropoxide. 


\subsection{Quantum chemical calculation}

Singles and doubles coupled cluster treatment using a cc-pVTZ basis set was employed for the $\tilde{X}^{2} A_{1}$ state. The JT interaction in the excited state $\tilde{A}^{2} E$ and the pJT coupling between the $\tilde{A}^{2} E$ and $\tilde{B}^{2} A_{1}$ states are treated using equation of motion electron excitation coupled-cluster theory (EOMEE-CCSD/cc-pVTZ) ${ }^{102} . C_{s}$ groups (an abelian subgroup of the $C_{3 v}$ group) are used as they transform as ${ }^{2} A^{\prime}$ and ${ }^{2} A^{\prime \prime}$. This method has been used for studying JT active and vibronic coupling cases for a number of molecules. The reference state for the EOMEE-CCSD calculation is the ground state of $\mathrm{CaOCH}\left(\mathrm{CH}_{3}\right)_{2}$.

Geometry optimization of the $\tilde{X}^{2} A_{1}$ and $\tilde{A}^{2} E$ states provide equilibrium bond lengths and bond angles, as well as rotational constants, of both states that are summarized in Table 1 . The largest change in geometry upon the $\tilde{A}_{1} / \tilde{A}_{2} \leftarrow \tilde{X}^{2}$ excitation is in the $\mathrm{CaO}$ bond length $\left(\Delta_{\mathrm{rCaO}} \sim 28 \mathrm{~m} \AA\right)$. Therefore, one expects vibronic transitions to the $\mathrm{CaO}$ stretch levels in the LIF/DF spectra, to be the strongest nondiagonal transitions. The vibrational assignment of LIF and DF spectra in the present work is guided by the quantum chemical calculations. $\mathrm{CaOCH}\left(\mathrm{CH}_{3}\right)_{2}$ has 30 vibrational modes, including 17 a' modes and 13 a' modes. Calculation of FC factors for both absorption and emission transitions under the harmonic oscillator approximation was performed using the ezSpectrum software.31 The LIF spectrum of the $\tilde{A}_{1} / \tilde{A}_{2} \leftarrow \tilde{X}$

transition and the DF spectrum of the $\tilde{A}_{1} / \tilde{A}_{2} \rightarrow \tilde{X}$ transition were simulated using the calculated vibrational frequencies and FC factors (See figure 1a,1b, 2a and 2b for CCSD simulations). 
In simulating the LIF spectrum, the vibrational temperature is set to $50 \mathrm{~K}$. The Gaussian line shape with a full-width at half-maximum (FWHM) of $5 \mathrm{~cm}^{-1}$ and $25 \mathrm{~cm}^{-1}$ was used to generate the simulated LIF and DF spectra, respectively ${ }^{3,102}$.

\subsection{Results}

\subsubsection{LIF spectrum}

The experimental LIF spectrum of the $\tilde{A}_{1} / \tilde{A}_{2} \leftarrow \tilde{X}$ transition of $\mathrm{CaOCH}\left(\mathrm{CH}_{3}\right)_{2}$ (Figure 1c) features six doublets with a frequency interval of $\sim 68 \mathrm{~cm}^{-1}$ between each pair of peaks. The strongest doublet centered at $\sim 15844 \mathrm{~cm}^{-1}$, is assigned to the origin transition, with the lower and higher frequency peaks assigned to the $\tilde{A}_{1}^{2} A^{\prime} \leftarrow \tilde{X}^{2} A^{\prime}\left(15878 \mathrm{~cm}^{-1}\right)$ and the $\tilde{A}_{2}^{2} A^{\prime \prime} \leftarrow \tilde{X}^{2} A^{\prime}\left(15810 \mathrm{~cm}^{-1}\right)$ transitions, respectively. By comparing to the simulation in terms of both vibrational frequencies and transition intensities, doublets centered at 318, 443, and $556 \mathrm{~cm}^{-1}$ to the blue of the origin band can be assigned to transitions to the $v^{\prime}=1$ levels of the $\mathrm{CaO}$ stretch combined with $\mathrm{CCCH}$ waggling $\left(15_{0}^{1}\right)$, COCC umbrella mode $\left(14_{0}^{1}\right)$, and the CO stretch $\left(13_{0}^{1}\right)$, respectively.

The weaker doublet pairs are observed centered at $83 \mathrm{~cm}^{-1}$ and $101 \mathrm{~cm}^{-1}$ blue of the origin band. The doublet centered at $101 \mathrm{~cm}^{-1}$ comparing with the simulation can be assigned as $\mathrm{CaOC}$ bending in-plane $\left(30_{0}^{1}\right)$. Another doublet centered at $83 \mathrm{~cm}^{-1}$ which is not predicted in the simulation can be most reasonable assigned as $\mathrm{CaOC}$ bending out of a plane. The doublet results from the splitting of the vibrational transition into two SO levels similar to the other lines in the spectrum. Due to its vibrational symmetry, transitions between the $17^{1}$ level of the $\tilde{A}$ state and the vibrationless ground level of the 
$\tilde{X}^{2} A^{\prime}$ state is forbidden. However, such transitions often gain intensity through vibronic interactions. In chapters 3 and 4 the vibronic transition has been discussed. In the LIF spectra, one big issue that has been introduced in chapter 3 and chapter 5 is the overestimation of the intensity of the forbidden transition and underestimation of the origin band. A separate cavity ring-down spectroscopy measurement (figure-6.5) has been performed to find out the extent of saturation. The intensity estimation by ab-initio calculation requires more work. For Ca-isopropoxide to improve the simulation the incorporation of vibronic coupling with ab-initio calculation is mandatory. You are still working on the calculation. However, the current chapter is showing a tentative assignments. 


\subsubsection{DF spectra obtained by pumping the origin transitions}

Figure 2c illustrates the DF spectrum obtained by pumping the $\tilde{A}_{1}^{2} A^{\prime} \rightarrow \tilde{X}^{2} A^{\prime}$ transition of $\mathrm{CaOCH}\left(\mathrm{CH}_{3}\right)_{2}$. In addition to the strong origin band, all observed peaks can be assigned to transitions to the $v^{\prime \prime}=1$ levels of the $\mathrm{CaO}$ stretch combined with $\mathrm{CCCH}$ waggling $\left(v_{15}\right)$, COCC umbrella mode $\left(v_{14}\right)$, and the CO stretch $\left(v_{13}\right)$. The peak at 1102 $\mathrm{cm}^{-1}$ is assigned to the $11_{1}^{0}$ transition which corresponds to CO stretch by comparing with the simulation. Another weak spectral feature has been observed around $98 \mathrm{~cm}^{-1}$ which by comparing it with simulation can be assigned as $\mathrm{CaOC}$ bending out of plane mode $\left(v_{30}\right)$. The DF spectrum obtained by pumping the $\tilde{A}_{2}^{2} A^{\prime \prime} \rightarrow \tilde{X}^{2} A^{\prime}$ transition (Figure 2d) also contains the aforementioned peaks. However, it has additional spectral features. First, the strongest peaks $\left(0_{0}^{0}, 15_{1}^{0}, 14_{1}^{0}\right)$ that are present in the DF spectrum obtained when pumping the $\tilde{A}_{2}^{2} A^{\prime \prime} \rightarrow \tilde{X}^{2} A^{\prime}$ transition are all accompanied by a weaker "child peak" to the red in the DF spectrum. The separation between the parent and child peaks is $\sim 68$ $\mathrm{cm}^{-1}$, identical to the SO splitting of the vibronic levels of the $\tilde{A}$ state. The presence of these "child peaks" is therefore ascribed to collision-induced population transfer from the upper $\tilde{A}_{2}^{2} A^{\prime \prime}$ SO level to the lower $\tilde{A}_{1}^{2} A^{\prime}$ SO level ${ }^{29}$. The subsequent $\tilde{A}_{1}^{2} A^{\prime} \rightarrow \tilde{X}^{2} A^{\prime}$ transitions are red-shifted relative to their counterparts in the $\tilde{A}_{2}^{2} A^{\prime \prime} \rightarrow \tilde{X}^{2} A^{\prime}$ transition, i.e., their "parent peaks", and the magnitude of the red-shift is equal to the SO splitting of the $\tilde{A}$ state. A band appeared around $1182 \mathrm{~cm}^{-1}$ which is the combination of $30_{0}^{1}$ and $17_{0}^{1}$ modes. The observation of a combination band in DF spectra is the effect of dushinski mixing that has been described in chapter 3 . 


\subsection{DF spectra obtained by pumping other vibronic bands}

DF spectra obtained by pumping strong vibronic bands in the LIF spectrum, namely, $17_{0}^{1}, 30_{0}^{1}, 15_{0}^{1}, 14_{0}^{1}$ and $13_{0}^{1}$, are illustrated in Figure 3b-f. Compared to those obtained by pumping the origin band (Figure 5a), DF transitions from a vibronic level of the $\tilde{A}$ state to those $\tilde{X}^{2} A^{\prime}$-state vibrational levels of the pumped mode gain intensity thanks to larger FC factors. Additionally, transitions to combination levels of the pumped

mode and other modes may also gain intensities because of the Duschinsky mixing. ${ }^{88,89}$ A simple technique that reveals this relation is to blue-shift DF spectra by the groundstate frequencies of the pumped modes. The resulting spectra are shown in Figure 4. When the $17_{0}^{1}$ (CaOC bending in-plane) band in the LIF spectrum is pumped, the DF spectrum contains, among others, a peak at $590 \mathrm{~cm}^{-1}$, designated by an "X" in Figure 5b. This peak defies any reasonable assignment to transitions of $\mathrm{CaOCH}_{3}$ especially when one considers that it is absent in the DF spectra obtained by pumping the $17_{0}^{1}$ band another component (see Figure 5d). It is probably due to impurity. Similarly, another peak in the spectrum obtained by pumping the $4_{0}^{1}$ band, labeled "Y" in Figure $5 c$ cannot be assigned either and also is attributed to impurity. 


\subsection{Conclusion}

We report vibrationally resolved LIF and DF spectra of the $\tilde{A}_{1} / \tilde{A}_{2} \leftarrow \tilde{X}$ transition of the calcium methoxide radical. The vibrational assignment has been made on the basis of CASSCF- and CC-calculated vibrational frequencies and FC factors for the allowed vibronic transitions. It has been found that both $a b$ initio methods predict the vibrational frequencies to a significant degree of accuracy. These calculations also do a reasonably good job for the intensity of the allowed, strong transitions. However other forbidden transitions are weakly observed in the spectrum which is not predicted to have intensity by either calculation. In the present work, relative intensities were determined in LIF and DF measurements, but our measurements have two limitations. First, the fluorescence signal is contaminated by the excitation laser scattering. Second, the excitation transitions of the origin band are power saturated. The second issue could be largely avoided by using continuous-wave (cw) excitation laser sources.

A separate cavity-ring down experiment has been performed to compensate these two issues by making use of the jet-cooled pulsed-laser CRD spectra. The signal-tonoise ratio of the current CRD measurement is somewhat lower than the LIF experiment. It can be significantly improved if cw laser sources are used. 
Table 6.1: Geometric parameters, rotational constants, and $\tilde{A}-\tilde{X}$ excitation energy of the $\mathrm{CaOCH}\left(\mathrm{CH}_{3}\right)_{2}$ radicals.

\begin{tabular}{|c|c|c|}
\hline & \multicolumn{2}{|c|}{$\begin{array}{c}\text { CCSD/EOM- } \\
\text { CCSD/cc-PVTZ }\end{array}$} \\
\hline & $\begin{array}{c}\widetilde{\boldsymbol{X}} \\
\text { state }\end{array}$ & $\begin{array}{c}\widetilde{\boldsymbol{A}}_{1} / \widetilde{\boldsymbol{A}}_{2} \\
\text { state }\end{array}$ \\
\hline $\mathrm{rCaO}(\AA)$ & 2.120 & 2.092 \\
\hline $\mathrm{rCO}(\AA)$ & 1.421 & 1.429 \\
\hline $\mathrm{rCC}(\AA)$ & 1.535 & 1.534 \\
\hline$\angle \mathrm{CaOC}(\mathrm{deg})$. & 179 & 179 \\
\hline$\angle \mathrm{OCC}(\mathrm{deg})$. & 110 & 110 \\
\hline $\mathrm{A}\left(\mathrm{cm}^{-1}\right)$ & .047 & .047 \\
\hline $\mathrm{B}\left(\mathrm{cm}^{-1}\right)$ & .267 & .267 \\
\hline $\mathrm{C}\left(\mathrm{cm}^{-1}\right)$ & & \\
\hline$\Delta E^{\tilde{A}_{1} / \tilde{A}_{2} \leftarrow \tilde{X}}\left(\mathrm{~cm}^{-}\right.$ & & \\
$11)$ & & \\
\hline
\end{tabular}

${ }^{\mathrm{a}}$ Compared to the experiment value of $15844 \mathrm{~cm}^{-1}$ 
(c)

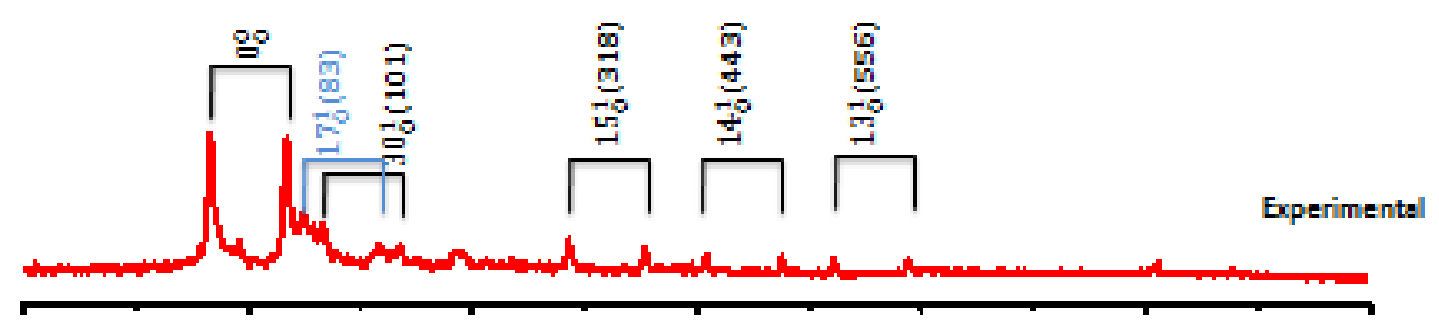

(b)

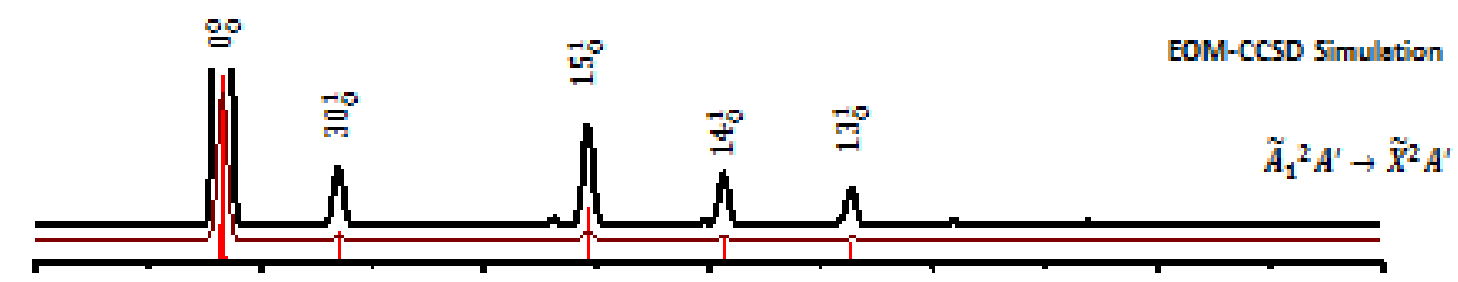

(a)

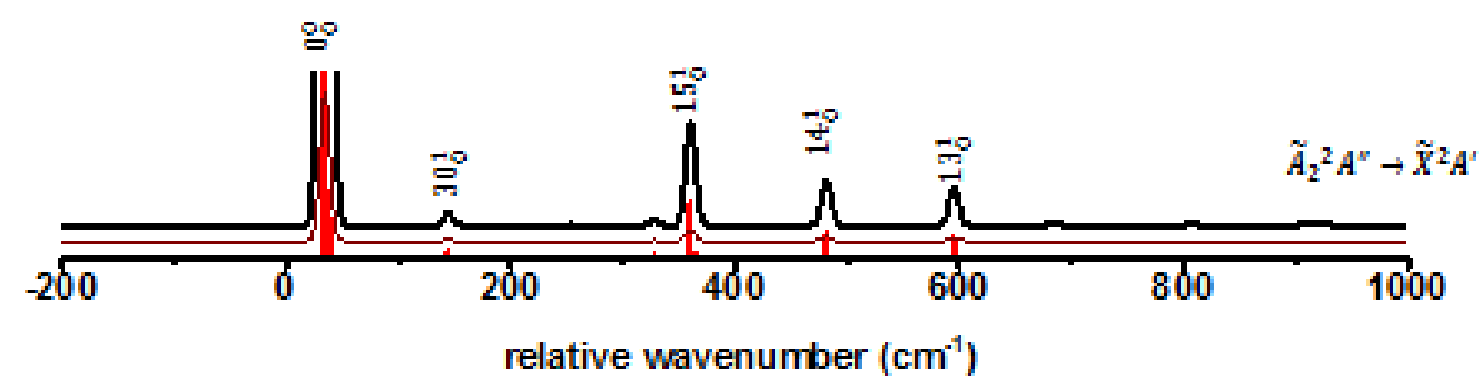

Figure 6.1: a and b is simulated for the $\tilde{A}_{1}^{2} A^{\prime} \leftarrow \tilde{X}^{2} A^{\prime}$ and $\tilde{A}_{2}^{2} A^{\prime \prime} \leftarrow \tilde{X}^{2} A^{\prime}$ transition of Ca-isopropoxide. Wavenumbers are relative to the origin band at $15844 \mathrm{~cm}^{-1}$. Numbers in parentheses are relative wavenumbers in $\mathrm{cm}^{-1}$. 


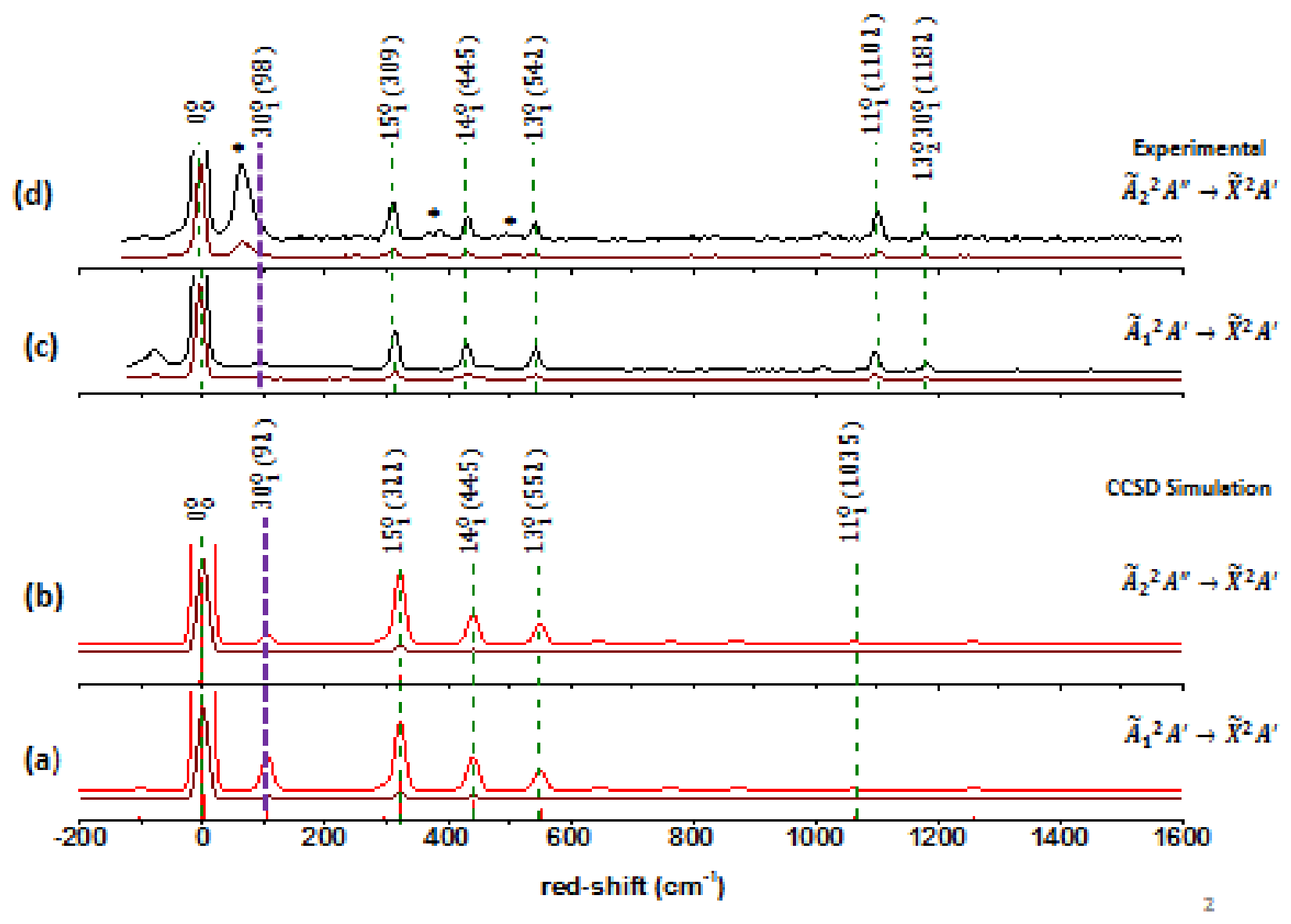

Figure 6.2: (a and b) Simulated for the $\tilde{A}_{1}^{2} A^{\prime} \rightarrow \tilde{X}^{2} A^{\prime}$ and $\tilde{A}_{2}^{2} A^{\prime \prime} \rightarrow \tilde{X}^{2} A^{\prime}$ transition of Ca-isopropoxide. DF spectra by pumping the (c) $\tilde{A}_{1}^{2} A^{\prime} \leftarrow \tilde{X}^{2} A^{\prime}$ and (d) $\tilde{A}_{2}^{2} A^{\prime \prime} \leftarrow \tilde{X}^{2} A^{\prime}$ origin transitions. Numbers in the parentheses are red-shift in $\mathrm{cm}-1$. The asterisked peaks in (d) are due to collision-induced population transfer. 


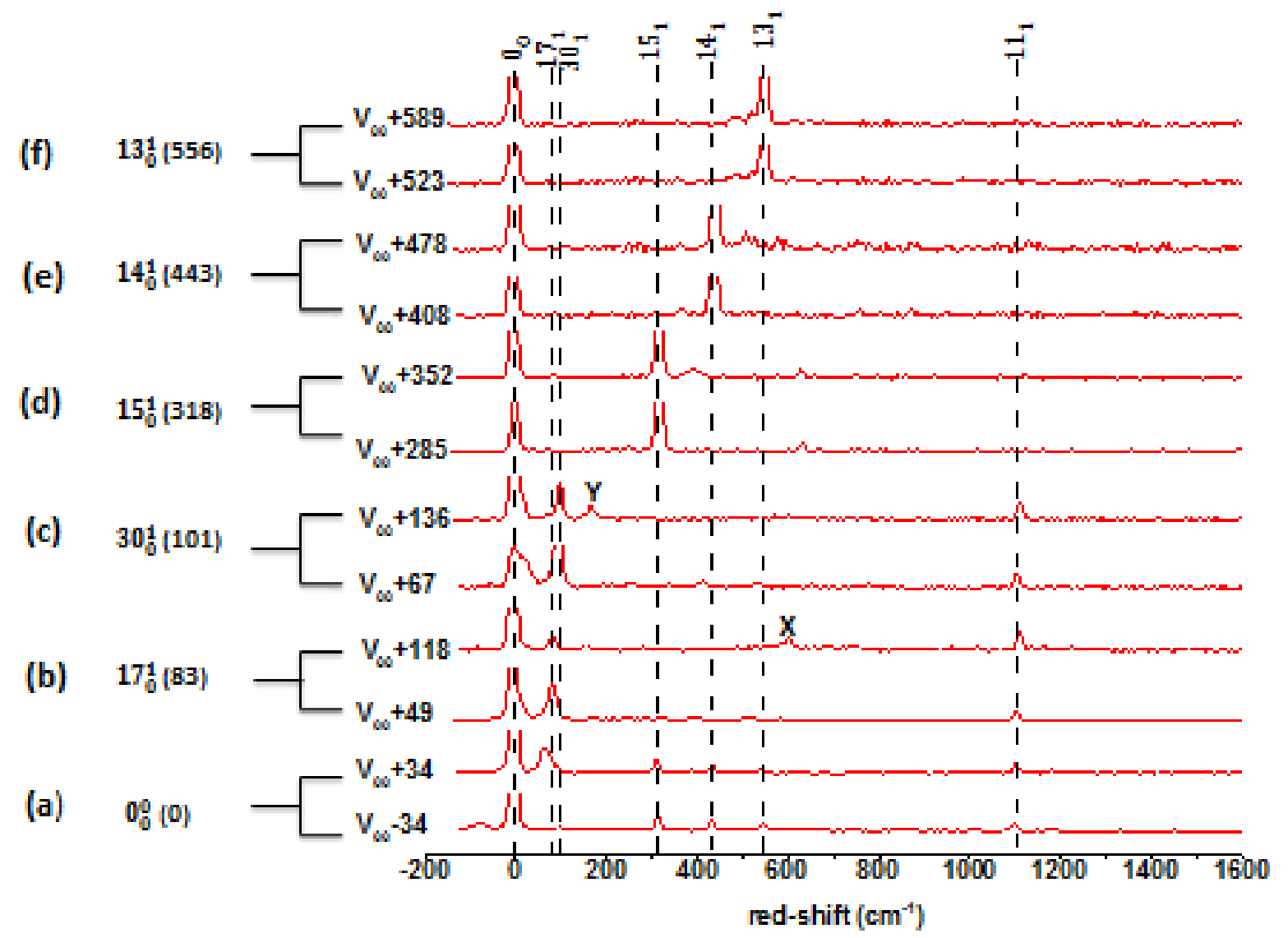

Figure 6.3: DF spectra by pumping different vibronic bands of the $\tilde{A}_{1} / \tilde{A}_{2} \leftarrow \tilde{X}$ transitions of ca-isopropoxide. Numbers in the parentheses are red-shift in $\mathrm{cm}^{-1}$. " $\mathrm{X}$ " and "Y" denotes unidentified transitions. 


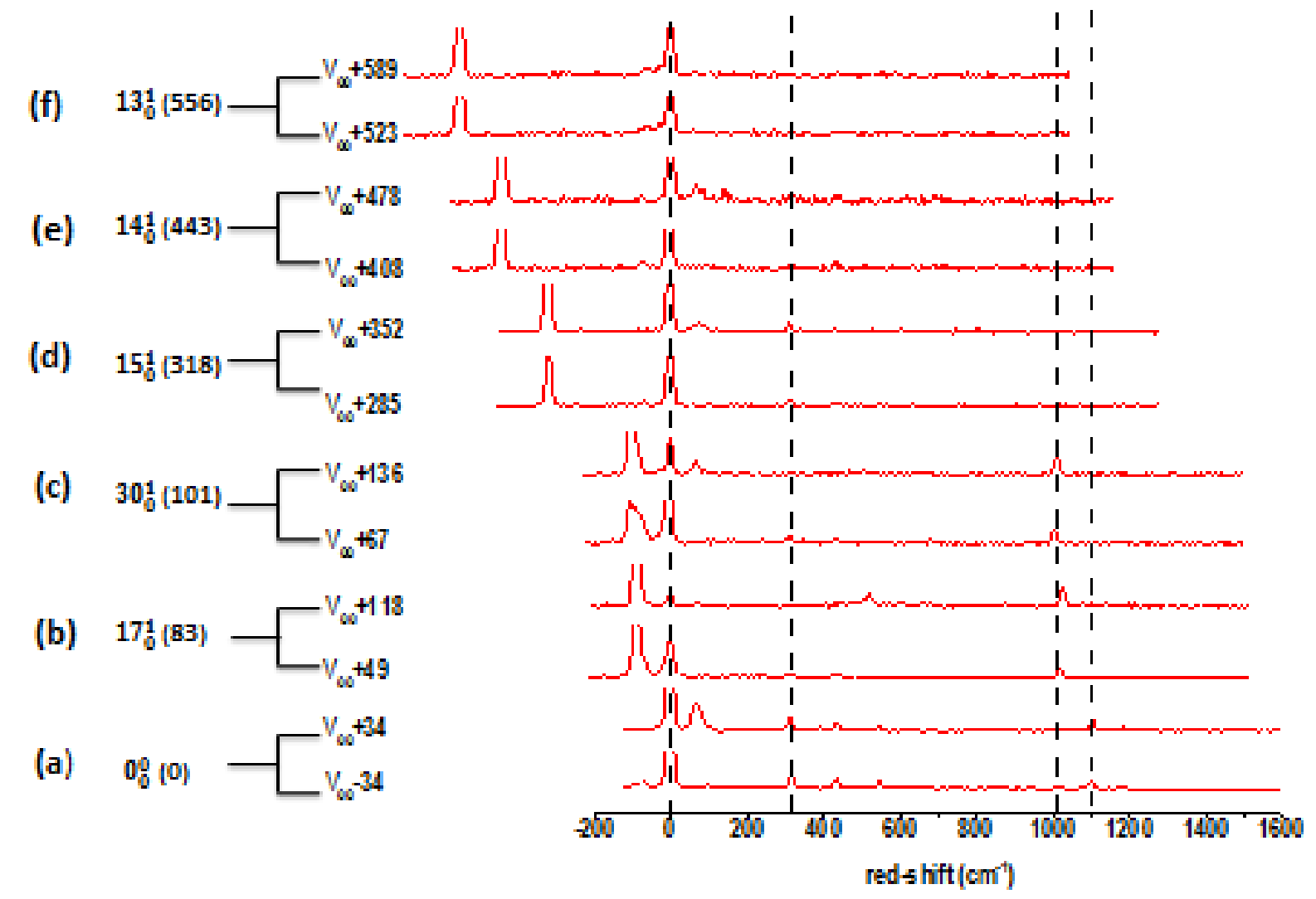

Figure 6.4: DF spectra by pumping different vibronic bands of the $\tilde{A}_{1} / \tilde{A}_{2} \leftarrow \tilde{X}$ transitions of ca-isopropoxide. Numbers in the parentheses are red-shift in $\mathrm{cm}^{-1}$. Spectra of bands other than origin band in the LIF spectrum are blue-shifted. 


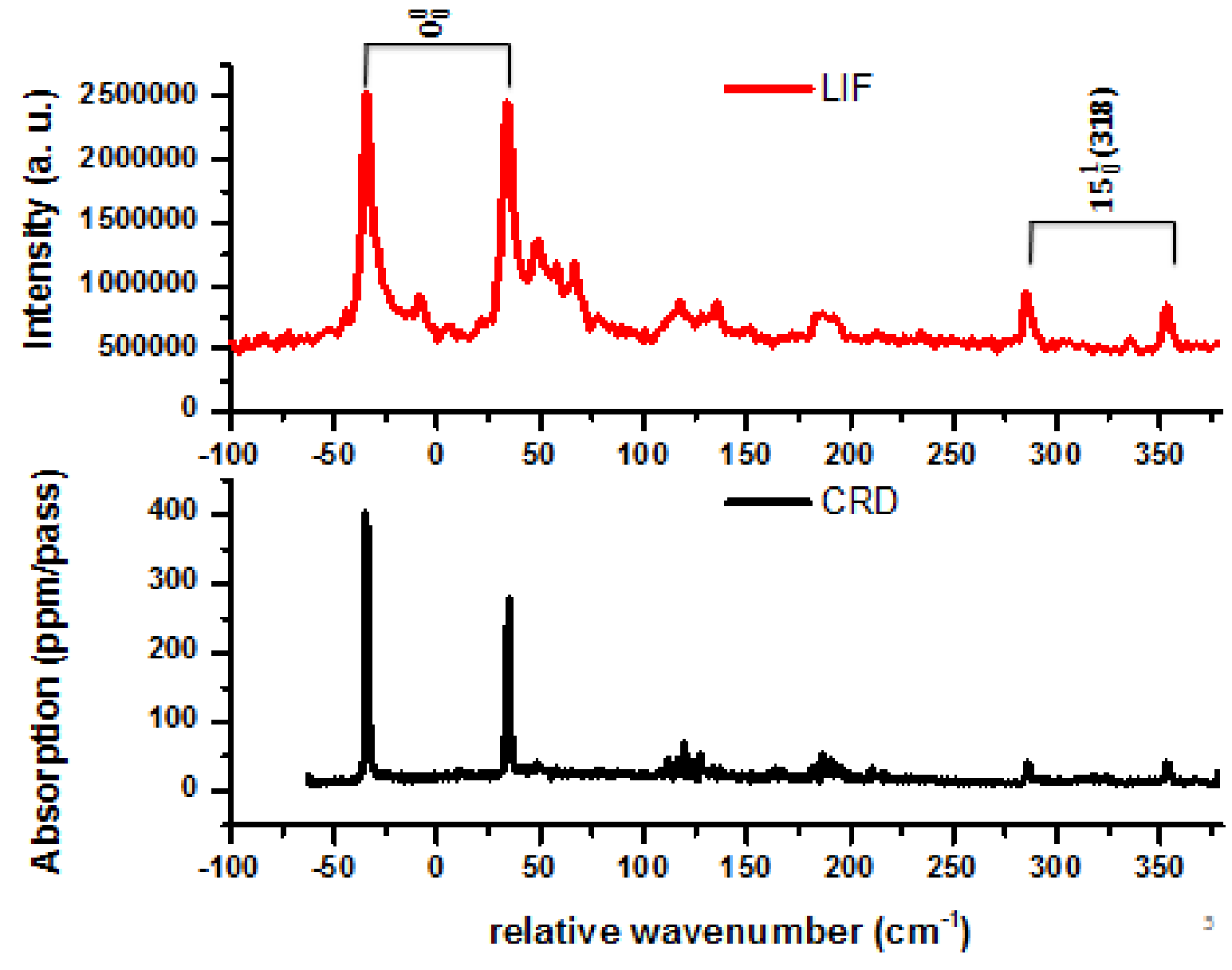

Figure 6.5: Comparison of (a) LIF and (b) CRD spectra of the origin and $15^{1}$ band transitions of $\mathrm{CaOCH}\left(\mathrm{CH}_{3}\right)_{2}$ that shows the power saturation of the origin transitions in the LIF experiment. 


\section{CHAPTER VII}

\section{CAVITY RING-DOWN SPECTROSCOPY OF JET-COOLED \\ YTTRIUM OXIDE (YO)}

Spectroscopic studies on yttrium oxide (YO) have a special interest in both astrophysics and chemical physics since the first study and analysis of the visible spectrum of the yttrium oxide (YO) molecule in $1930 \mathrm{~s}^{103,104}$. YO has long been known to astrophysicists as the observational detection of its optical absorption spectrum has been suggested as a key indicator in cool stars ${ }^{105}$. The spectroscopic data determined from laboratory experiments, $A^{2} \Pi-X^{2} \Sigma^{+}$and $B^{2} \Sigma^{+}-X^{2} \Sigma^{+}$electronic transition system in the orange and blue regions have used to interpret local physical and chemical processes in various objects. Besides the interest of studying $\mathrm{YO}$ in astrophysics, it has also been suggested as one of the benched marked molecules used for laser cooling and trapping. $\mathrm{YO}$ is one of the diatomic molecules that have been laser-cooled. One of the important criteria for choosing a candidate molecule for laser cooling is the absence of any intermediate states between two involve electronic states. In a laser cooling, presence of any intermediate state especially if there is any "dark state" terminate the cooling cycle by population leakage. Past investigations of electronic structure analysis by Uhler and Akerland $^{106}$ concluded that the spin splitting in the $X^{2} \Sigma^{+}$state was negligible and the spin-orbit splitting in the $A^{2} \Pi$ state was $428 \mathrm{~cm}^{-1}$ and the two spin orbit states are 
$A^{2} \Pi_{1 / 2}$ and $A^{2} \Pi_{3 / 2}$ where $\Pi_{3 / 2}$ component is higher in energy. Initially, the $A^{2} \Pi_{1 / 2}-$ $X^{2} \Sigma^{+}$electronic transition of YO used for laser cooling and the cooling temperature was as low as $2 \mathrm{mK}$ in a 2D-magneto optical trapping (MOT) by Jun Ye group. The ${ }^{7}$, ${ }^{107}$ population leakage to the $A^{\prime} \Delta_{3 / 2}$ of YO was limiting the cooling temperature. By rotational state microwave mixing $\mathrm{Ye}^{7}$ group was able to minimize the population loss. Recently, the same research group achieved cooling temperature down to $10 \mu \mathrm{K}$ by using the $A^{\prime} \Delta_{3 / 2}-X^{2} \Sigma^{+}$dipole-forbidden transition of YO.

The $X^{2} \Sigma^{+}, A^{2} \Pi, A^{\prime 2} \Delta_{3 / 2}, B^{2} \Sigma^{+}$and $D^{2} \Sigma^{+}$electronic states have been known for YO molecules and have been studied in jet-cooled or room-temperature conditions either using fluorescence or absorption spectroscopy. The $B^{2} \Sigma^{+}$had been studied using cavity ring-down spectroscopy by Cheung at $\mathrm{al}^{108}$. To our knowledge, there is no direct absorption spectroscopy for $A^{2} \Pi$ state or $A^{\prime 2} \Delta_{3 / 2}$ for YO. The advantage of cavity ring down spectroscopy over fluorescence spectroscopy is that fluorescence is a two steps process and easily the transition can be saturated in the process whereas cavity ring down is a single step. With cavity ring-down spectroscopy, intensity can be measured much accurately as in this technique the optical saturation is negligible due to relatively weak inter-cavity laser field and insignificant effects of excitation laser scattering ${ }^{102}$. We used cavity ring-down spectroscopy in an aim to study the $A^{\prime 2} \Delta_{3 / 2}$ and $A^{\prime 2} \Delta_{5 / 2}$ state of this molecule.

In the present work, we report the cavity ring-down spectra of both $A^{2} \Pi_{1 / 2}-X^{2} \Sigma^{+}$and $A^{2} \Pi_{3 / 2}-X^{2} \Sigma^{+}$transition of YO under jet-cooled conditions. 
Accurate molecular constants for $A^{2} \Pi$ state has determined by fitting the experimentally observed transition using p-gopher.

\subsection{Experimental}

The laser vaporization free-jet expansion and the cavity ring-down laser absorption apparatus used in this study have been described in detail in our earlier publications. Here, we used a similar chamber and nozzle as LIF described above in order to obtain jet cooled CRD rather than room temperature. YO molecules were generated by $1064 \mathrm{~nm}$ laser ablation of a yttrium rod (ESPI metals) in the presence of the mixture of oxygen and helium gas. The signal was optimized using $8 \%$ oxygen with helium. Helium carrier gas provides lower vibrational temperature ${ }^{65}$. We also tried with argon carrier gas and different percentage of oxygen. With 8\% oxygen and helium carrier gas, we observed the best spectrum in terms of $\mathrm{S} / \mathrm{N}$ ratio.

CRD mirrors (Los Gatos research, R> 99.995\%. center wavelength at $620 \mathrm{~nm}$ ) were mounted on the arms of the vacuum chamber to form a ring-down cavity and they were purged by $\mathrm{N}_{2}$ flow continuously to prevent contaminations. A similar laser that had been used in LIF experiment was used as a CRD laser. The transmission of the CRD laser was focused on a photomultiplier tube (PMT, Hamamatsu, and H10721-01). The signal was acquired with an oscilloscope (Tektronix, DPO3014). The empty cavity ring-down time was about 50us. Typically, the ring-down curves were averaged 64 times at each laser wavelength before being sent to a PC via a USB port. The averaged ringdown curves of up to 100,000 data points were fit to a bi-exponential decay function. The 
longer of the two fit time constants was used to calculate the absorption (in ppm/pass). Ring-down signal was collected at each wavelength with the ablation laser on and off. The exposition of the ablation laser on the rotating metal rod continuously generates metal vapor which contaminates the optical cavity. The on and off of the ablation laser was used to get rid of the excess metal vapor produced inside the chamber by increasing the delay time allowing the pump to suck the unreacted metal vapor before the next acquisition event. The LabVIEW program controlled the on/off of the ablation laser and the wavelength scan of the dye laser. The experimental apparatus was synchronized by a pulse delay generator (Stanford Research System, DG645) at a 20Hz repetition rate. With the pulsed pinhole expansion aforementioned the path length of the absorber where the ring-down laser exposed was about $1 \mathrm{~cm}$. To increase the length of the absorber we used the slit-jet expansion which increases the length to $4 \mathrm{~cm}$.

Our slit-jet laser ablation expansion is an alteration from the design of Terry Miller group ${ }^{109}$. Figure 7.1 representing the slit-jet nozzle. The solenoid mount and the slit body were made from aluminum. A $5.5 \mathrm{~cm}$ long aluminum poppet is actuated by two commercial general valve solenoids with a multi-channel nozzle driver circuit (General Valve Corporation, Iota one). A Viton (durometer 65A) sealing cord is trapped within the poppet. Pulsed laser ablation method was used to generate radicals in the free jet expansion. A metal holder body with the identical dimensions of the slit body placed after the slit body. The metal holder was designed in such a way that it can hold a metal of $6 \mathrm{~mm}$ width and $50 \mathrm{~mm}$ long. A gear motor (spark fun) is mounted on the side of the metal body that can hold the yttrium metal rod on the shaft of the motor. To maintain the stability of the signal the metal rod was rotated steadily with $10 \mathrm{rpm}$, so each laser shot 
ablates a fresh spot on the surface of the $\operatorname{rod}^{65}$. The rotational speed was maintained by a homemade simple electrical circuit with a potentiometer (100 KQ⿱) $)$. The potentiometer was used for the variables rotation control by changing the pulse width. The slit on the front face of the slit body has the dimensions $(50 \times .5 \times 1 \mathrm{~mm})$. The $300 \mu \mathrm{m}$ jet width of the jet throat is defined by the blades jaw. 


\subsection{Results and Discussion}

\subsubsection{Electronic structure of YO}

The presence of ${ }^{2} \Delta$ state in $A-X$ system makes the electronic structure of YO complicated. It was shown ${ }^{105}$ that the intensity of the $A^{\prime 2} \Delta-X^{2} \Sigma^{+}$transitions arose as a result of considerable mixing between $A^{\prime 2} \Delta$ and $A^{2} \Pi$ states. The $A-X$ and $B-X$ system of YO lie in the orange-red and blue-green regions respectively, and the $A^{\prime}-X$ system is in the $620-747 \mathrm{~nm}$ region. Recently, $D^{2} \Sigma^{+}-X^{2} \Sigma^{+}$electronic transitions have been observed in the 400-440 $\mathrm{nm}$ regions. For the observation of these electronic states mostly fluorescence spectroscopy has been used so far. Only the $B^{2} \Sigma^{+}-X^{2} \Sigma^{+}$systems have been studied using cavity ring down spectroscopy. The complicated structure of YO with laser cooling scheme and the summery of reported vibrational constants can be found in figure 7.2 and table 7.1 respectively. Among all this transition $A-X$ system of YO has been studied by several groups. Linton et $\mathrm{al}^{105}$ reported the photoluminescence spectra of two strong sequences of bands, starting at $597.2 \mathrm{~nm}$ and $613.2 \mathrm{~nm}$ are representing the $\Delta v=0$ sequences originating from the ${ }^{2} \Pi_{3 / 2}$ and ${ }^{2} \Pi_{5 / 2}$ components respectively.

The potential curves of the two-state aforementioned lie vertically above each other which makes the $\Delta v=1$ transitions very weak. The rotational structure of the $0-0$ band with 4-4 band head had been reported by Linton et al ${ }^{105}$. 


\subsubsection{Rotational assignment of the bands}

The bands of the ${ }^{2} \Pi \leftarrow{ }^{2} \Sigma^{+}$system are the most intense in the $\Delta v=0$ sequence. The system is including two series of sub bands of almost equal intensity. The separation between the series is $429.4690 \mathrm{~cm}^{-1}$ which corresponding to the $1 / 2$ and $3 / 2$ components of the ${ }^{2} \Pi$ state. Because of the large separation between the sub bands, it is clear that $A^{2} \Pi$ state belongs to Hund's case (a), so each sub band is comprised of six branches ${ }^{110}$. In the present case, as the spin-splitting constant in $X^{2} \Sigma^{+}$state is very small so only four branches are plausible. Figure 4.3 representing the $A^{2} \Pi_{3 / 2} \leftarrow$ $X^{2} \Sigma^{+}$transitions. For the ${ }^{2} \Pi_{3 / 2} \leftarrow{ }^{2} \Sigma^{+}$sub band the branches are designated as $P_{2}$, ${ }^{Q} P_{21}$ $+Q_{2},{ }^{R} Q_{21}+R_{2},{ }^{S} R_{21}$ and their respective intensities are in the ratios of 1:3:3:1 for the same $J$ " which is consistent with the reported emission spectra ${ }^{110}$. Figure 4.4 representing the $A^{2} \Pi_{1 / 2} \leftarrow X^{2} \Sigma^{+}$transitions In the similar manner for the ${ }^{2} \Pi_{1 / 2} \leftarrow{ }^{2} \Sigma^{+}$ sub band four branches ${ }^{O} P_{12},{ }^{P} Q_{12}+P_{1},{ }^{Q} R_{12}+Q_{1}$ and $R_{1}$ have been observed with the same intensities' ratio. Observed lines positions and branches are given in table 4.3 and 4.4. Rotational analysis confirms that all these observed lines are to a regular $A^{2} \Pi$ state. This observation is a very good agreement with the previously reported FTIR spectrum. The author reported room temperature spectra of both components of $A \rightarrow X$ and $B \rightarrow X$ . They mentioned the perturbations in the $(2,2)$ band of the $A \rightarrow X$ system. The dominant perturbations are in the $\mathrm{Q}_{2}$ branches, especially in the $A^{2} \Pi_{3 / 2}(v=0)$ state. The author claims that the perturbing state is probably the $A^{\prime 2} \Delta$ state which lies in the vicinity ( $T_{e} \approx 14700 \mathrm{~cm}^{-1}$ ) of the A state. In our observed spectrum for both $\Pi_{1 / 2}$ and $\Pi_{3 / 2}$ component, 
we have some of the extra lines which cannot be assigned with fitted spectra using pure $A^{2} \Pi$ state. These lines might be the transitions due to the perturbation of the $A^{\prime 2} \Delta$ state. 


\subsection{Effective Hamiltonian}

The analysis of the rotational structure of diatomic molecules ${ }^{2} \Sigma$ and ${ }^{2} \Pi$ can be found elsewhere ${ }^{110}$. Here we give a brief summary of the Hamiltonians we used for the fitting. The effective Hamiltonian for a ${ }^{2} \Sigma$ state can be written as following:

$$
\widehat{H}_{2_{\Sigma}}=\widehat{H}_{R O T}+\widehat{H}_{C D}+\widehat{H}_{S R}
$$

Where $\widehat{H}_{R O T}$ represents the rotational part of the Hamiltonian, $\widehat{H}_{C D}$ corresponds to the centrifugal distortion and $\widehat{H}_{S R}$ is the spin-rotation part of the Hamiltonian.

And the effective Hamiltonian for $\mathrm{a}^{2} \Pi$ can be expressed as:

$$
\widehat{H}_{2 \Sigma}=\widehat{H}_{R O T}+\widehat{H}_{S O}+\widehat{H}_{C D}+\widehat{H}_{2 \Lambda}
$$

Where $\widehat{H}_{S O}$ represents the spin-orbit and $\widehat{H}_{2 \Lambda}$ corresponds to the $\Lambda$-type doubling part of the Hamiltonian.

In case diatomic molecules Rotational Hamiltonian can be written as

$$
\widehat{H}_{R O T}=B \widehat{N}^{2}
$$

Where $\widehat{N}$ is the rotational angular momentum and B is the rotational constant, $N=J-$ $L-S$, where $J$ is the total angular momentum of the molecule, $L$ is the electron orbital angular momentum and $S$ is the electron spin angular momentum. 
As experimentally we observed lines of very high, it is necessary to include centrifugal distortion effects. Centrifugal distortion term in the Hamiltonian can be expressed as following:

$$
\widehat{H}_{C D}=D \widehat{N}^{4}
$$

where D is centrifugal distortion constant.

A very weak spin-rotation interaction had been included in the Hamiltonian for the better fitting. The spin-rotation Hamiltonian can be expressed as

$$
\widehat{H}_{S R}=\gamma \widehat{N} . \hat{S}
$$

where $\gamma$ is the spin-rotation constant.

For the ${ }^{2} \Pi$ state as we know that it has spin-orbit (SO) splitting. In order to fit the lines accurately, it was necessary to add SO interaction in the Hamiltonian. The SO Hamiltonian can be represented as

$$
\widehat{H}_{S O}=A \hat{L} . \hat{S}=A\left[L_{z} S_{z}+1 / 2\left(L_{+} S_{-}+L_{-} S_{+}\right)\right]
$$

Here A is the SO constant and $\left(L_{+} S_{-}+L_{-} S_{+}\right)$term becomes zero.

Finally, as the two SO components are separated by only about $429 \mathrm{~cm}^{-1}$ a $\Lambda$-type doubling which corresponds to the perturbation between two SO components had been added. The $\Lambda$-type doubling Hamiltonian can be expressed as

$$
\widehat{H}_{2 \Lambda}=1 / 2 \mu \hat{r}^{2}\left(J^{+} L^{-}+J^{-} L^{+}\right)
$$


Here, $\hat{r}$ is the internuclear distance operator and the $\Lambda$-type doubling has two components, 1) linear component (p) 2) quadratic component (q) that has been used in our fitting. 


\subsection{High-temperature spectra recorded using slit-jet expansion}

The slit-jet expansion has been discussed in section-. As stated above the absorber length getting 4 times by using slit jet expansion, it was expected that the signal to noise ratio will increase by an order of magnitude. But the observed spectra show different scenarios. The $\mathrm{S} / \mathrm{N}$ ratio was comparable with pin-hole expansion. We recorder the spectrum for $A^{2} \Pi_{3 / 2} \leftarrow X^{2} \Sigma^{+}$transition which is representing in figure 7.5 The recorded spectrum if we compare with the spectrum reported by FTIR emission spectra we can see that it has some similarity. The 4 branches are obvious with the absence of band head. The band head usually occurs at a very high temperature due to the overlap of many rotational lines at low resolution. Band head also occurs in vibration-rotation spectra, but as the difference between the ground and excited-state rotational constant is relatively small the head often occurs at sufficiently high $J$ that it escapes observation.

We did a simulation for this band and found the temperature is high about $400 \mathrm{~K}^{65}$. The possible reasons for getting the high-temperature spectrum in the experiment using slit are the collision among the radicals and between the metal body and the radicals were not sufficient enough to cool down and also as ablation laser was continuously heating on the surface of the nozzle body it was getting hot. 


\subsection{Improvement of the resolution and sensitivity}

The molecular constants in table 7.2 compare the fitted value from our experiment with the literature reported value. The transition frequency and the spin-orbit constant are much accurate in our data as we calibrated the frequency using wavemeter. All other constants are limited by the resolution of the current system.

With the current sensitivity of our system, it is almost impossible to study the

$A^{\prime 2} \Delta$ state. The current single to noise ratio is about 250 to 1 . For the $A^{\prime 2} \Delta$ state the transition intensity is expected to be about 50 times smaller than $A^{2} \Pi$ state. In that sense, the current $\mathrm{S} / \mathrm{N}$ ratio is not enough to detect the $A^{\prime 2} \Delta$ state. Pulsed cavity ring down method with narrower linewidth is a probable solution.

To gain the narrower linewidth Pulsed-dye amplifier (100 MHz) can be used. Another way to improve the resolution is CW laser. A ring-dye laser with $1 \mathrm{MHz}$ linewidth can be used. In our free jet expansion, the Doppler broadening is about 100 MHz. To beat the Doppler broadening it is better to use pulsed -dye amplifier.

As we know that $\mathrm{YO}$ is one of the diatomic molecules that have been laser cold and recently Jun ye group used $A^{\prime 2} \Delta-X^{2} \Sigma^{+}$transition for the cooling, it is important to determine the Franck-Condon factor accurately. We know that for accurate intensity measurement CRD is the best method but still in the CRD experiment a large error of intensity measurement is possible due to the fluctuations of the radical concentrations due to the pulse-to-pulse fluctuations of the ablation laser poser and its efficiency of ablation at different locations on the rotating yttrium metal rod. A twobeam experiment could a possible solution to get rid of the aforementioned problem. The 
diagram of the two-beam experiment is given in figure 7.6. In this experiment, a CRD laser will be used to record one of the specific transitions at a fixed frequency and another CRD laser will be used to scan other transitions. So, the intensity of the overall transition can be normalized using the fixed frequency transition intensity. 


\subsection{Conclusion}

This work presents the jet-cooled cavity ring-down spectra of $A^{2} \Pi_{1 / 2} \leftarrow$ $X^{2} \Sigma^{+}$and $A^{2} \Pi_{3 / 2} \leftarrow X^{2} \Sigma^{+}$transition for the first time by our group to the best of our knowledge. The molecular constants determined by fitting the experimental spectra are in considerable accuracy. The transitions that are not predicted in the simulation might be due to the perturbation of the $A^{\prime 2} \Delta$ state. As we know that our data is better calibrated with wave meter we can assure that the transition frequency and the spin-orbit constants are quite accurate. All other constants are mainly limited by the resolution of the system. Both sensitivity and the resolution can be improved by using a narrow linewidth laser source. 

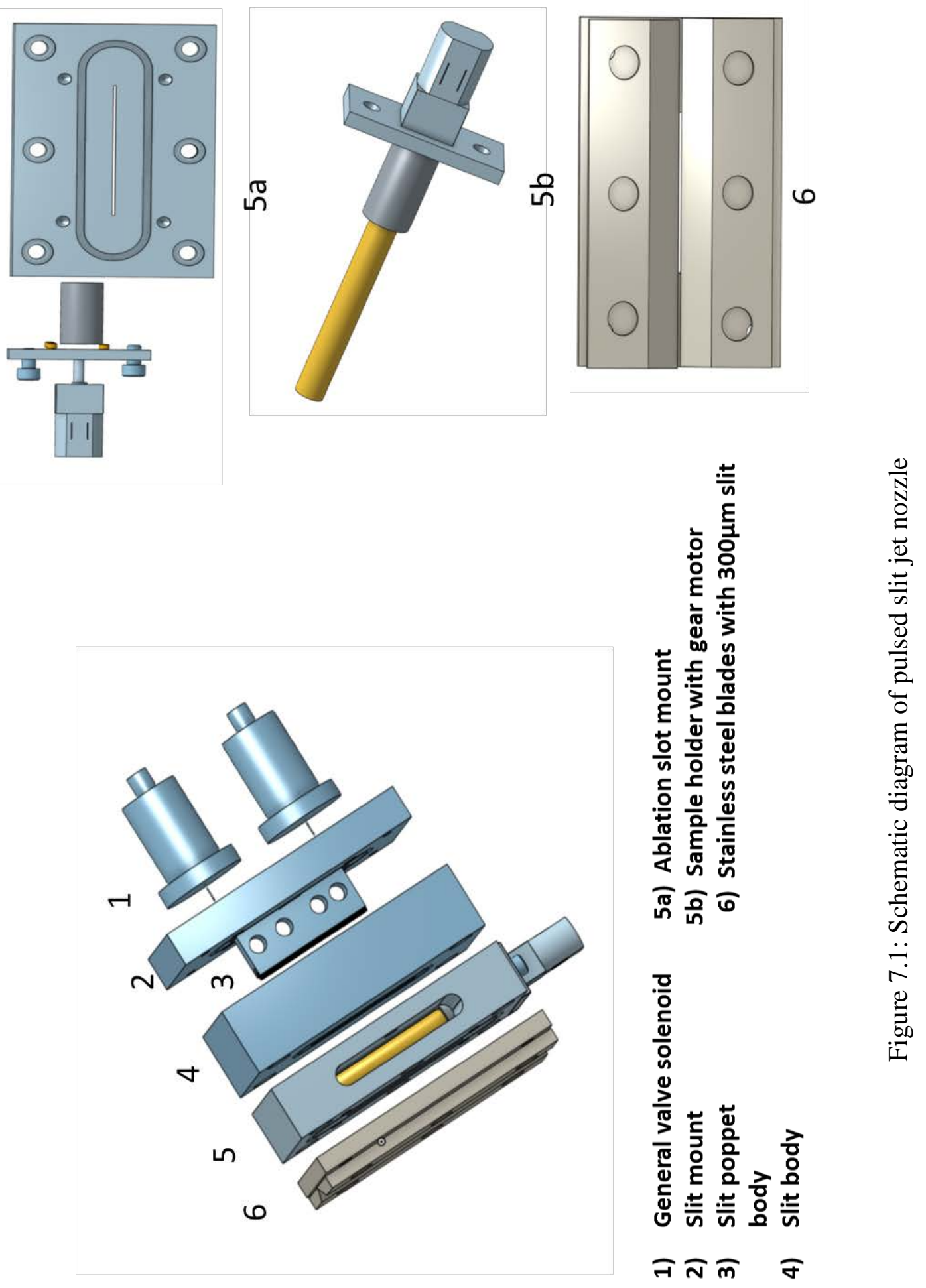


$$
\begin{aligned}
& \dot{v}=1 \frac{D^{2} \Sigma^{+}}{v=1} \\
& \dot{v}=B^{2} \Sigma^{+}
\end{aligned}
$$

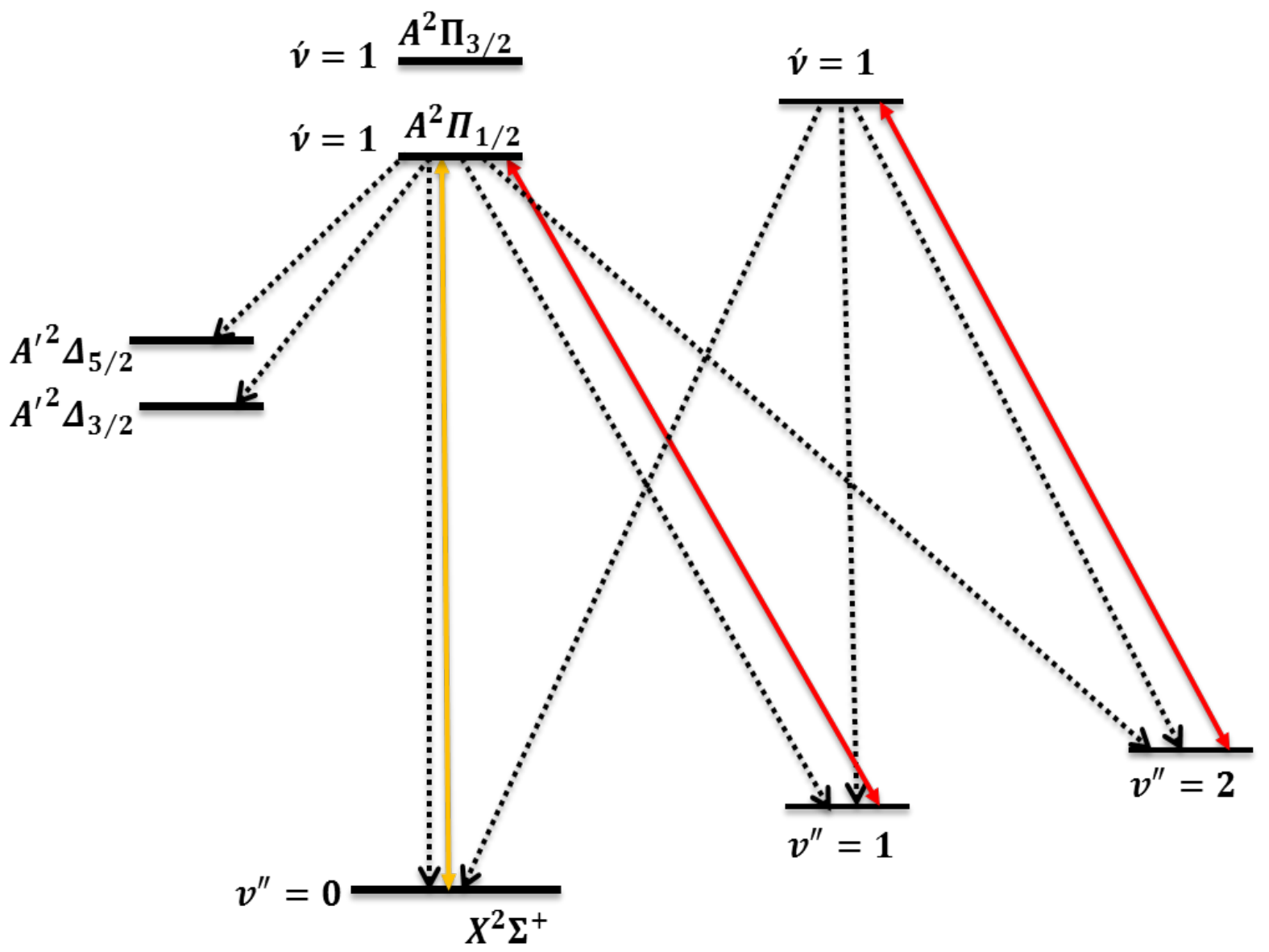

Figure 7.2: Electronic structure of YO. Solid arrows indicate cooling and re-pump laser transitions. Dashed arrows indicate decay paths. 


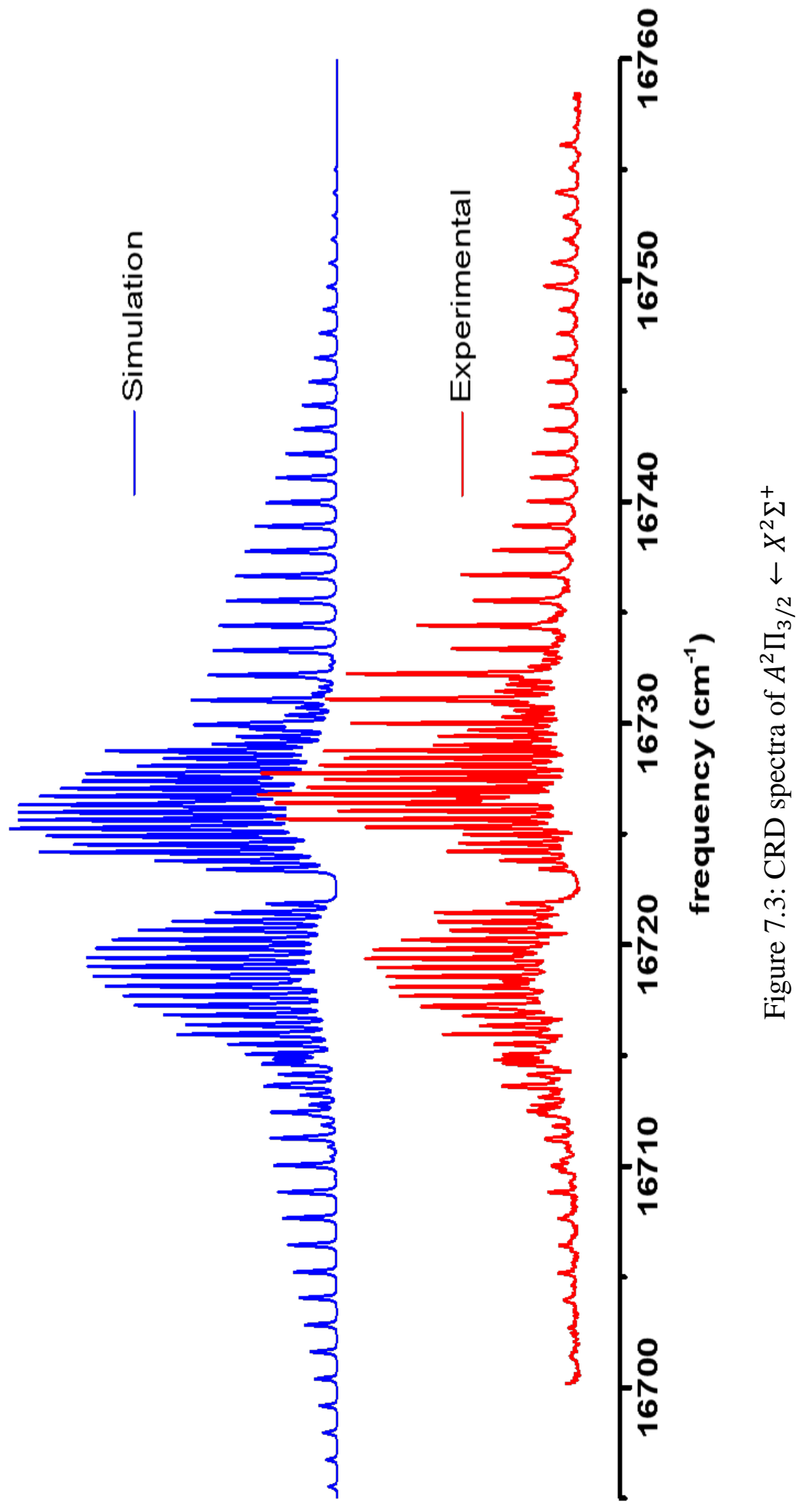




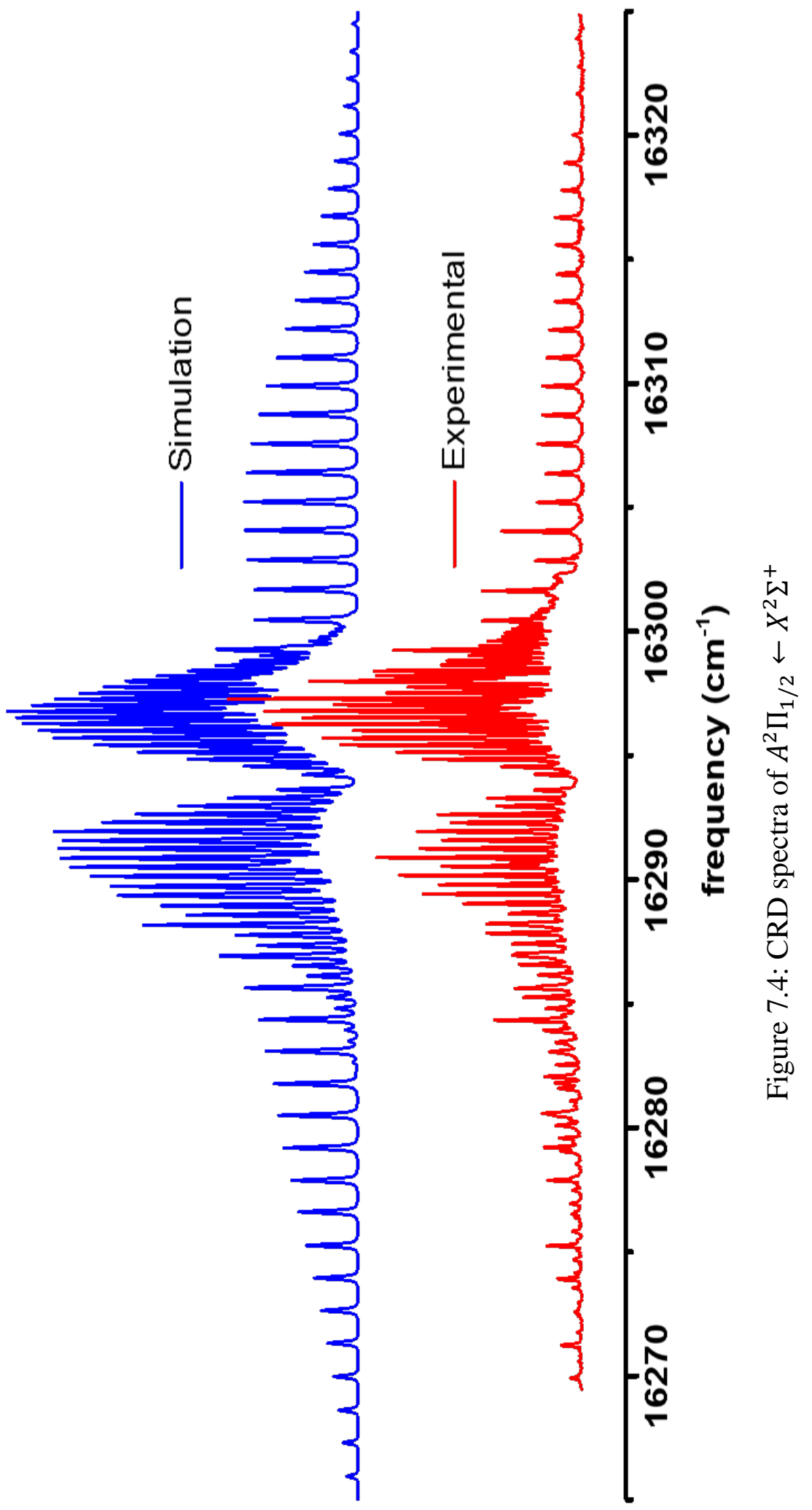




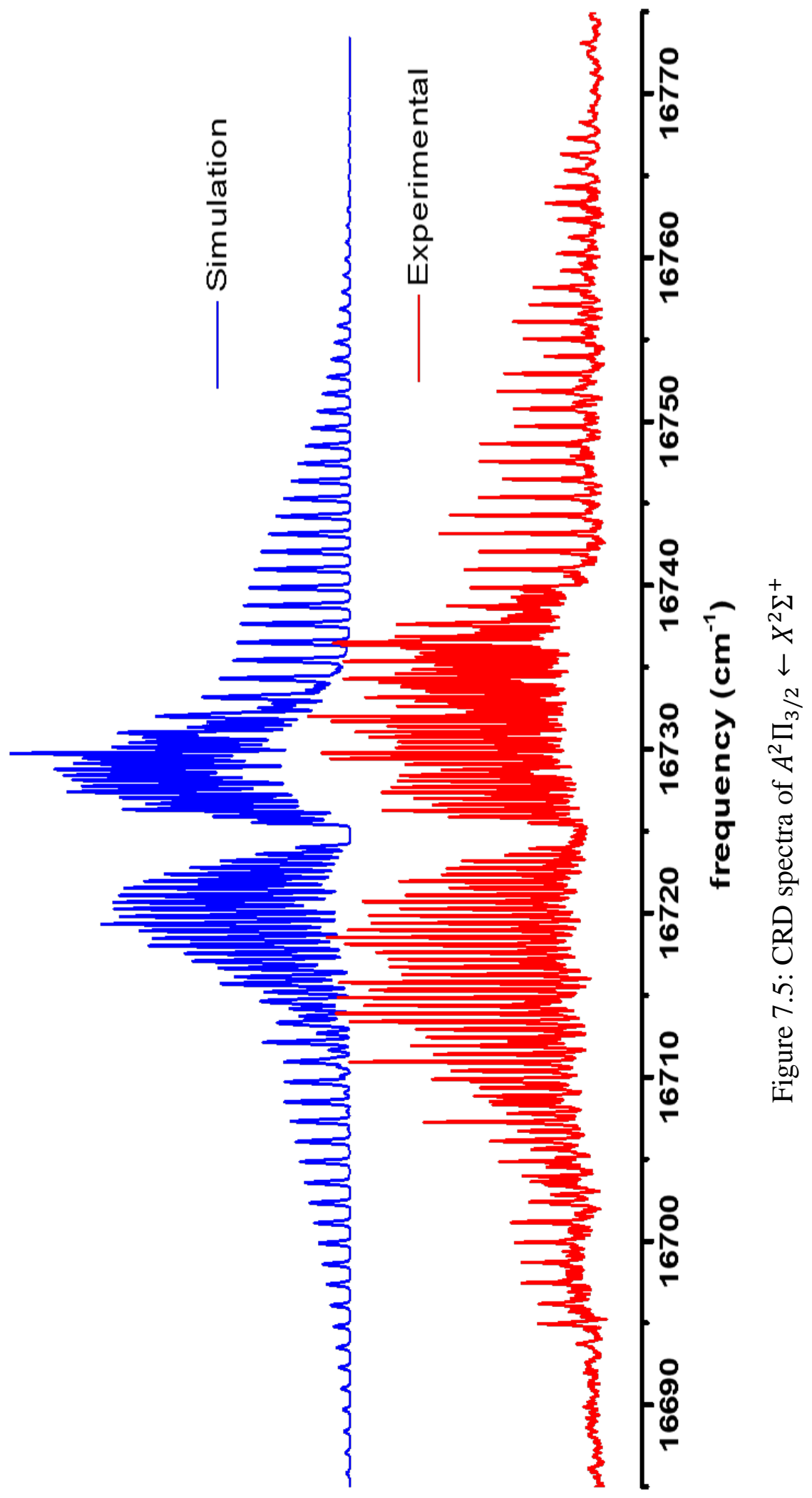




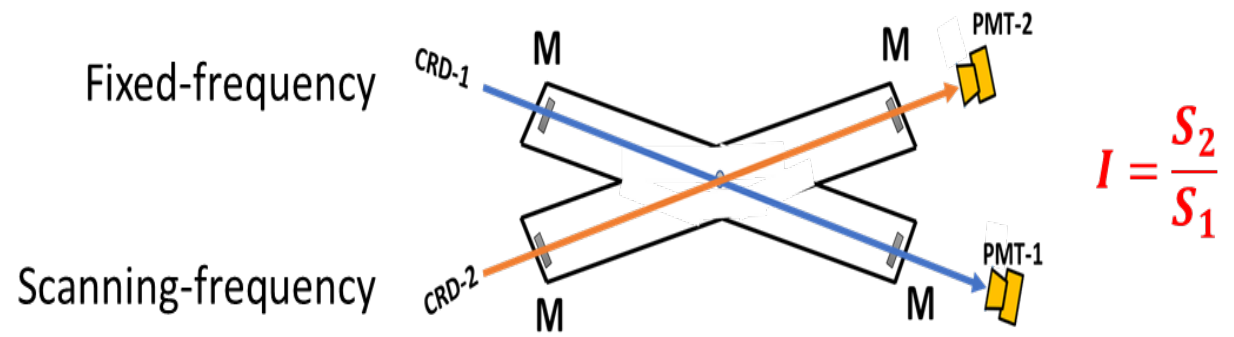

Figure 7.6: Schematic diagram of Two beam experiment. M: ring-down mirror, S1 : intensity of fixed frequency transition, S2 : Intensities of the transitions of scanning frequencies, I: normalized frequency 
Table 7.1: Electronic transition frequencies and vibrational constants of different states.

\begin{tabular}{|c|c|c|c|c|c|c|c|}
\hline State & $\mathrm{T}_{00}\left(\mathrm{~cm}^{-1}\right)$ & $\begin{array}{c}\omega_{\mathrm{e}} \\
\left(\mathrm{cm}^{-1}\right)\end{array}$ & $\begin{array}{c}\omega_{\mathrm{e}_{\mathrm{e}}} \\
\left(\mathrm{cm}^{-1}\right)\end{array}$ & $B_{e}\left(\mathrm{~cm}^{-1}\right)$ & $\alpha_{e}\left(\mathrm{~cm}^{-1}\right)$ & $\mathrm{R}_{\mathrm{e}}(\AA)$ & $\begin{array}{l}\text { Experimental } \\
\text { Technique }\end{array}$ \\
\hline$D^{2} \Sigma^{+}$ & 23969.94 & & & 0.339578 & & 1.91 & LIF \\
\hline \multirow{2}{*}{$B^{2} \Sigma^{+}$} & 20741.69 & 765.5 & & 0.3731897 & & & CRDS \\
\hline & 20791 & 765.5 & 8 & 0.3722 & & 1.8279 & Emission \\
\hline \multirow{3}{*}{$A^{2} \Pi_{3 / 2}$} & 16746.8 & 820 & 3.38 & 0.3865 & \begin{tabular}{|l|}
0.0019 \\
\end{tabular} & & Emission \\
\hline & & & & 0.3868 & 0.0020 & & $\begin{array}{l}\text { Photo- } \\
\text { luminescence }\end{array}$ \\
\hline & 16742.2 & 822.7 & 3.9 & 0.3857 & 0.0023 & 1.7956 & \\
\hline \multirow{3}{*}{$A^{2} \Pi_{1 / 2}$} & 16315.8 & 823 & 3.45 & 0.3865 & \begin{tabular}{|l|}
0.0019 \\
\end{tabular} & & \\
\hline & & & & 0.3868 & 0.0020 & & \\
\hline & 16315 & 820.7 & 3.5 & 0.3857 & 0.0023 & 1.7956 & \\
\hline$A^{\prime 2} \Delta_{5 / 2}$ & 14870.4 & 794.9 & 3.3 & & & & $\begin{array}{c}\text { Chemi- } \\
\text { luminescence }\end{array}$ \\
\hline$A^{\prime 2} \Delta_{3 / 2}$ & 14531.2 & 794 & 3.23 & & & & \\
\hline \multirow{5}{*}{$x^{2} \Sigma^{+}$} & 0 & 862 & 2.86 & 0.3892 & 0.0016 & & \\
\hline & 0 & 862.1 & 3.025 & & & & \\
\hline & 0 & 861 & 2.93 & 0.3881 & 0.0018 & 1.79 & LIF \\
\hline & 0 & 861.46 & & & & & \\
\hline & 0 & 861 & 2.93 & 0.3881 & \begin{tabular}{|l|}
0.0018 \\
\end{tabular} & 1.79 & \\
\hline
\end{tabular}


Table 7.2 : Comparison of molecular constants wit literature reported values

\begin{tabular}{|c|c|c|c|}
\hline \multicolumn{5}{|c|}{$A^{2} \Pi$ - state } \\
\hline Constants $\left(\mathrm{cm}^{-1}\right)$ & Presentwork & Ref-1 & Ref-2 \\
\hline$T_{00}$ & $16508.2914(53)$ & $16509.06254(40)$ & $16518.984(22)$ \\
\hline$B$ & $0.385654(242)$ & $0.3857644(88)$ & $0.385776(53)$ \\
\hline$D \times 10^{6}$ & $0.881(35)$ & $0.3408(18)$ & $0.3468(27)$ \\
\hline$A$ & $429.4690(85)$ & $428.61128(79)$ & $408.757(42)$ \\
\hline$A_{0} \times 10^{4}$ & $4.37(35)$ & & $-0.14969(33)$ \\
\hline$p$ & $-0.1567(10)$ & $-0.150374(42)$ & \\
\hline$q \times 10^{3}$ & $5.86(65)$ & $0.1265(66)$ & $0.388092(54)$ \\
\hline & & $X^{2} \Sigma^{+}-$state & $0.3188(28)$ \\
\hline$B$ & $0.388097(249)$ & $0.3880382(77)$ & \\
\hline$D \times 10^{6}$ & $1.15(37)$ & $0.3147(17)$ & \\
\hline$V \times 10^{4}$ & $0.527(37)$ & $0.49(19)$ & \\
\hline
\end{tabular}

1. A. Bernard, R. Bacis, and P. Luc, The Astrophys. J. 227, 338-348 (1979)

2. C. Linton, J. Mol. Spec. 69, 351-364 (1978) 
Table 7.3: Rotational line positions and branches for $A^{2} \Pi_{3 / 2} \leftarrow X^{2} \Sigma^{+}$transition

\begin{tabular}{|c|c|c|c|}
\hline $\mathbf{J}^{\prime}$ & $\mathbf{J}^{\prime \prime}$ & Position & Branch \\
\hline 7.5 & 8.5 & 16710.1588 & $\mathrm{P}_{2}(8.5)$ \\
\hline 8.5 & 9.5 & 16708.9085 & $\mathrm{P}_{2}(9.5)$ \\
\hline 10.5 & 11.5 & 16708.8202 & $\mathrm{P}_{2}(11.5)$ \\
\hline 11.5 & 12.5 & 16707.6581 & $\mathrm{P}_{2}(12.5)$ \\
\hline 12.5 & 13.5 & 16706.4148 & $\mathrm{P}_{2}(13.5)$ \\
\hline 13.5 & 14.5 & 16705.1787 & $\mathrm{P}_{2}(14.5)$ \\
\hline 14.5 & 15.5 & 16703.9605 & $\mathrm{P}_{2}(15.5)$ \\
\hline 1.5 & 2.5 & 16721.8386 & ${ }^{\mathrm{Q}} \mathrm{P}_{21}+\mathrm{Q}_{2}(2.5)$ \\
\hline 2.5 & 3.5 & 16721.4572 & ${ }^{\mathrm{Q}} \mathrm{P}_{21}+\mathrm{Q}_{2}(3.5)$ \\
\hline 3.5 & 4.5 & 16721.0358 & ${ }^{{ }^{Q}} P_{21}+Q_{2}(4.5)$ \\
\hline 4.5 & 5.5 & 16720.6184 & ${ }^{\mathrm{Q}} \mathrm{P}_{21}+\mathrm{Q}_{2}(5.5)$ \\
\hline 5.5 & 6.5 & 16720.2235 & ${ }^{{ }} P_{21}+Q_{2}(6.5)$ \\
\hline 6.5 & 7.5 & 16719.8028 & ${ }^{\mathrm{Q}} \mathrm{P}_{21}+\mathrm{Q}_{2}(7.5)$ \\
\hline 7.5 & 8.5 & 16719.3736 & ${ }^{\mathrm{Q}} \mathrm{P}_{21}+\mathrm{Q}_{2}(8.5)$ \\
\hline 8.5 & 9.5 & 16718.9545 & ${ }^{\mathrm{Q}} \mathrm{P}_{21}+\mathrm{Q}_{2}(9.5)$ \\
\hline 9.5 & 10.5 & 16718.5357 & ${ }^{\mathrm{Q}} \mathrm{P}_{21}+\mathrm{Q}_{2}(10.5)$ \\
\hline 10.5 & 11.5 & 16718.1108 & ${ }_{\mathrm{Q}} \mathrm{P}_{21}+\mathrm{Q}_{2}(11.5)$ \\
\hline 11.5 & 12.5 & 16717.6728 & ${ }^{\mathrm{Q}} \mathrm{P}_{21}+\mathrm{Q}_{2}(12.5)$ \\
\hline 12.5 & 13.5 & 16717.2384 & ${ }^{\mathrm{Q}} \mathrm{P}_{21}+\mathrm{Q}_{2}(13.5)$ \\
\hline
\end{tabular}




\begin{tabular}{|c|c|c|c|}
\hline 13.5 & 14.5 & 16716.7975 & ${ }^{\mathrm{Q}} \mathrm{P}_{21}+\mathrm{Q}_{2}(14.5)$ \\
\hline 14.5 & 15.5 & 16716.3705 & ${ }^{Q} P_{21}+Q_{2}(15.5)$ \\
\hline 15.5 & 16.5 & 16715.9375 & ${ }^{\mathrm{Q}} \mathrm{P}_{21}+\mathrm{Q}_{2}(16.5)$ \\
\hline 16.5 & 17.5 & 16715.4841 & ${ }^{\mathrm{Q}} \mathrm{P}_{21}+\mathrm{Q}_{2}(17.5)$ \\
\hline 17.5 & 18.5 & 16715.0417 & ${ }^{\mathrm{Q}} \mathrm{P}_{21}+\mathrm{Q}_{2}(18.5)$ \\
\hline 18.5 & 19.5 & 16714.5803 & ${ }^{2} \mathrm{P}_{21}+\mathrm{Q}_{2}(19.5)$ \\
\hline 19.5 & 20.5 & 16714.1056 & ${ }^{\mathrm{Q}} \mathrm{P}_{21}+\mathrm{Q}_{2}(20.5)$ \\
\hline 20.5 & 21.5 & 16713.6170 & ${ }^{\mathrm{Q}} \mathrm{P}_{21}+\mathrm{Q}_{2}(21.5)$ \\
\hline 1.5 & 1.5 & 16723.4380 & ${ }^{\mathrm{R}} \mathrm{Q}_{21}+\mathrm{R}_{2}(2.5)$ \\
\hline 2.5 & 2.5 & 16723.8252 & ${ }^{\mathrm{R}} \mathrm{Q}_{21}+\mathrm{R} 2(3.5)$ \\
\hline 3.5 & 3.5 & 16724.2224 & ${ }^{\mathrm{R}} \mathrm{Q}_{21}+\mathrm{R}_{2}(4.5)$ \\
\hline 4.5 & 4.5 & 16724.5854 & ${ }^{\mathrm{R}} \mathrm{Q}_{21}+\mathrm{R}_{2}(5.5)$ \\
\hline 5.5 & 5.5 & 16724.9484 & ${ }^{\mathrm{R}} \mathrm{Q}_{21}+\mathrm{R}_{2}(6.5)$ \\
\hline 6.5 & 6.5 & 16725.3266 & ${ }^{\mathrm{R}} \mathrm{Q}_{21}+\mathrm{R}_{2}(7.5)$ \\
\hline 7.5 & 7.5 & 16725.6900 & ${ }^{\mathrm{R}} \mathrm{Q}_{21}+\mathrm{R}_{2}(8.5)$ \\
\hline 8.5 & 8.5 & 16726.0476 & ${ }^{\mathrm{R}} \mathrm{Q}_{21}+\mathrm{R}_{2}(9.5)$ \\
\hline 9.5 & 9.5 & 16726.4168 & ${ }^{\mathrm{R}} \mathrm{Q}_{21}+\mathrm{R}_{2}(10.5)$ \\
\hline 10.5 & 10.5 & 16726.7669 & ${ }^{\mathrm{R}} \mathrm{Q}_{21}+\mathrm{R}_{2}(11.5)$ \\
\hline 11.5 & 11.5 & 16727.1019 & ${ }^{\mathrm{R}} \mathrm{Q}_{21}+\mathrm{R}_{2}(12.5)$ \\
\hline 12.5 & 12.5 & 16727.4435 & ${ }^{\mathrm{R}} \mathrm{Q}_{21}+\mathrm{R}_{2}(13.5)$ \\
\hline 13.5 & 13.5 & 16727.7384 & ${ }^{\mathrm{R}} \mathrm{Q}_{21}+\mathrm{R}_{2}(14.5)$ \\
\hline & & & \\
\hline
\end{tabular}




\begin{tabular}{|c|c|c|c|}
\hline 10.5 & 9.5 & 16734.4057 & ${ }^{\mathrm{s}} \mathrm{R}_{21}(9.5)$ \\
\hline 11.5 & 10.5 & 16735.5377 & ${ }^{S} \mathrm{R}_{21}(10.5)$ \\
\hline 12.5 & 11.5 & 16736.6950 & ${ }^{\mathrm{S}} \mathrm{R}_{21}(11.5)$ \\
\hline 13.5 & 12.5 & 16737.8056 & ${ }^{\mathrm{S}} \mathrm{R}_{21}(12.5)$ \\
\hline 14.5 & 13.5 & 16738.9042 & ${ }^{\mathrm{S}} \mathrm{R}_{21}(13.5)$ \\
\hline 15.5 & 14.5 & 16740.0200 & ${ }^{\mathrm{s}} \mathrm{R}_{21}(14.5)$ \\
\hline 16.5 & 15.5 & 16741.0943 & ${ }^{s} \mathrm{R}_{21}(15.5)$ \\
\hline 17.5 & 16.5 & 16742.1909 & ${ }^{\mathrm{S}} \mathrm{R}_{21}(16.5)$ \\
\hline 18.5 & 17.5 & 16743.2489 & ${ }^{s} \mathrm{R}_{21}(17.5)$ \\
\hline 19.5 & 18.5 & 16744.3242 & ${ }^{S} \mathrm{R}_{21}(18.5)$ \\
\hline 20.5 & 19.5 & 16745.4187 & ${ }^{\mathrm{S}} \mathrm{R}_{21}(19.5)$ \\
\hline 21.5 & 20.5 & 16746.4931 & ${ }^{\mathrm{S}} \mathrm{R}_{21}(20.5)$ \\
\hline 22.5 & 21.5 & 16747.5969 & ${ }^{\mathrm{S}} \mathrm{R}_{21}(21.5)$ \\
\hline 23.5 & 22.5 & 16748.6602 & ${ }^{\mathrm{S}} \mathrm{R}_{21}(22.5)$ \\
\hline 24.5 & 23.5 & 16749.7035 & ${ }^{\mathrm{S}} \mathrm{R}_{21}$ (23.5) \\
\hline 25.5 & 24.5 & 16750.7809 & ${ }^{s} \mathrm{R}_{21}(24.5)$ \\
\hline 26.5 & 25.5 & 16751.8229 & ${ }^{\mathrm{S}} \mathrm{R}_{21}(25.5)$ \\
\hline 27.5 & 26.5 & 16752.8793 & ${ }^{\mathrm{S}} \mathrm{R}_{21}$ (26.5) \\
\hline 28.5 & 27.5 & 16753.9681 & ${ }^{\mathrm{S}} \mathrm{R}_{21}$ (27.5) \\
\hline
\end{tabular}


Table 7.4: Rotational line positions and branches for $A^{2} \Pi_{1 / 2} \leftarrow X^{2} \Sigma^{+}$transition

\begin{tabular}{|c|c|c|c|}
\hline $\mathbf{J}^{\prime}$ & J" & Position & Branch \\
\hline 10.5 & 11.5 & 16279.21 & $\mathrm{P}_{12}(11.5)$ \\
\hline 11.5 & 12.5 & 16277.89 & $\mathrm{P}_{12}(12.5)$ \\
\hline 12.5 & 13.5 & 16276.38 & $\mathrm{P}_{12}(13.5)$ \\
\hline 13.5 & 14.5 & 16275.25 & $\mathrm{P}_{12}(14.5)$ \\
\hline 14.5 & 15.5 & 16273.91 & $\mathrm{P}_{12}(15.5)$ \\
\hline 15.5 & 16.5 & 16273.55 & $\mathrm{P}_{12}(16.5)$ \\
\hline 16.5 & 17.5 & 16272.57 & $\mathrm{P}_{12}(17.5)$ \\
\hline 17.5 & 18.5 & 16271.23 & $\mathrm{P}_{12}(18.5)$ \\
\hline 18.5 & 19.5 & 16269.91 & $\mathrm{P}_{12}(19.5)$ \\
\hline 1.5 & 2.5 & 16293.28 & ${ }^{\mathrm{P}} \mathrm{Q}_{12}+\mathrm{P}_{1}(2.5)$ \\
\hline 2.5 & 3.5 & 16292.95 & ${ }^{\mathrm{P}} \mathrm{Q}_{12}+\mathrm{P}_{1}(3.5)$ \\
\hline 3.5 & 4.5 & 16292.62 & ${ }^{\mathrm{P}} \mathrm{Q} 12+\mathrm{P} 1(4.5)$ \\
\hline 4.5 & 5.5 & 16292.29 & ${ }_{\mathrm{PQ}} \mathrm{Q}_{12}+\mathrm{P}_{1}(5.5)$ \\
\hline 5.5 & 6.5 & 16291.94 & ${ }^{\mathrm{P}} \mathrm{Q}_{12}+\mathrm{P}_{1}(6.5)$ \\
\hline 6.5 & 7.5 & 16291.59 & ${ }^{\mathrm{P}} \mathrm{Q}_{12}+\mathrm{P}_{1}(7.5)$ \\
\hline 7.5 & 8.5 & 16291.24 & ${ }^{\mathrm{P}} \mathrm{Q}_{12}+\mathrm{P}_{1}(8.5)$ \\
\hline 8.5 & 9.5 & 16290.89 & ${ }^{\mathrm{P}} \mathrm{Q}_{12}+\mathrm{P}_{1}(9.5)$ \\
\hline 9.5 & 10.5 & 16290.53 & ${ }^{\mathrm{P}} \mathrm{Q}_{12}+\mathrm{P}_{1}(10.5)$ \\
\hline 10.5 & 11.5 & 16290.16 & ${ }^{\mathrm{P}} \mathrm{Q}_{12}+\mathrm{P}_{1}(11.5)$ \\
\hline
\end{tabular}




\begin{tabular}{|c|c|c|c|}
\hline 11.5 & 12.5 & 16289.8 & ${ }^{\mathrm{P}} \mathrm{Q}_{12}+\mathrm{P}_{1}(12.5)$ \\
\hline 12.5 & 13.5 & 16289.42 & ${ }^{\mathrm{P}} \mathrm{Q}_{12}+\mathrm{P}_{1}(13.5)$ \\
\hline 13.5 & 14.5 & 16289.04 & ${ }^{\mathrm{P}} \mathrm{Q}_{12}+\mathrm{P}_{1}(14.5)$ \\
\hline 14.5 & 15.5 & 16288.65 & ${ }^{\mathrm{P}} \mathrm{Q}_{12}+\mathrm{P}_{1}(15.5)$ \\
\hline 15.5 & 16.5 & 16288.23 & ${ }^{\mathrm{P}} \mathrm{Q}_{12}+\mathrm{P}_{1}(16.5)$ \\
\hline 16.5 & 17.5 & 16287.83 & ${ }^{\mathrm{P}} \mathrm{Q}_{12}+\mathrm{P}_{1}(17.5)$ \\
\hline 17.5 & 18.5 & 16287.42 & ${ }^{\mathrm{P}} \mathrm{Q}_{12}+\mathrm{P}_{1}(18.5)$ \\
\hline 18.5 & 19.5 & 16286.98 & ${ }^{\mathrm{P}} \mathrm{Q}_{12}+\mathrm{P}_{1}(19.5)$ \\
\hline 19.5 & 20.5 & 16286.57 & ${ }^{\mathrm{P}} \mathrm{Q}_{12}+\mathrm{P}_{1}(20.5)$ \\
\hline 20.5 & 21.5 & 16286.15 & ${ }^{\mathrm{P}} \mathrm{Q}_{12}+\mathrm{P}_{1}(21.5)$ \\
\hline 21.5 & 22.5 & 16285.72 & ${ }^{\mathrm{P}} \mathrm{Q}_{12}+\mathrm{P}_{1}(22.5)$ \\
\hline 22.5 & 23.5 & 16285.26 & ${ }^{\mathrm{P}} \mathrm{Q}_{12}+\mathrm{P}_{1}(23.5)$ \\
\hline 23.5 & 24.5 & 16284.81 & ${ }^{\mathrm{P}} \mathrm{Q}_{12}+\mathrm{P}_{1}(24.5)$ \\
\hline 24.5 & 25.5 & 16284.34 & ${ }^{\mathrm{P}} \mathrm{Q}_{12}+\mathrm{P}_{1}(25.5)$ \\
\hline 25.5 & 26.5 & 16283.91 & ${ }^{\mathrm{P}} \mathrm{Q}_{12}+\mathrm{P}_{1}(26.5)$ \\
\hline 1.5 & 1.5 & 16294.55 & ${ }^{\mathrm{Q}} \mathrm{R}_{12}+\mathrm{Q}_{11}(1.5)$ \\
\hline 2.5 & 2.5 & 16294.85 & ${ }_{\mathrm{Q}} \mathrm{R}_{12}+\mathrm{Q}_{11}(2.5)$ \\
\hline 3.5 & 3.5 & 16295.14 & ${ }^{\mathrm{Q}} \mathrm{R}_{12}+\mathrm{Q}_{11}(3.5)$ \\
\hline 4.5 & 4.5 & 16295.42 & ${ }^{\mathrm{Q}} \mathrm{R}_{12}+\mathrm{Q}_{11}(4.5)$ \\
\hline 5.5 & 5.5 & 16295.71 & ${ }^{\mathrm{Q}} \mathrm{R}_{12}+\mathrm{Q}_{11}(5.5)$ \\
\hline 6.5 & 6.5 & 16296 & ${ }^{\mathrm{Q}} \mathrm{R}_{12}+\mathrm{Q}_{11}(6.5)$ \\
\hline 7.5 & 7.5 & 16296.27 & ${ }^{\mathrm{Q}} \mathrm{R}_{12}+\mathrm{Q}_{11}(7.5)$ \\
\hline
\end{tabular}




\begin{tabular}{|c|c|c|c|}
\hline 8.5 & 8.5 & 16296.53 & ${ }^{\mathrm{Q}} \mathrm{R}_{12}+\mathrm{Q}_{11}(8.5)$ \\
\hline 9.5 & 9.5 & 16296.8 & ${ }^{\mathrm{Q}} \mathrm{R}_{12}+\mathrm{Q}_{11}(9.5)$ \\
\hline 10.5 & 10.5 & 16297.04 & ${ }^{Q} \mathrm{R}_{12}+Q_{11}(10.5)$ \\
\hline 11.5 & 11.5 & 16297.28 & ${ }^{\mathrm{Q}} \mathrm{R}_{12}+\mathrm{Q}_{11}(11.5)$ \\
\hline 12.5 & 12.5 & 16297.53 & ${ }^{\mathrm{Q}} \mathrm{R}_{12}+\mathrm{Q}_{11}(12.5)$ \\
\hline 13.5 & 13.5 & 16297.76 & ${ }^{\mathrm{Q}} \mathrm{R}_{12}+\mathrm{Q}_{11}(13.5)$ \\
\hline 14.5 & 14.5 & 16298 & ${ }^{\mathrm{Q}} \mathrm{R}_{12}+\mathrm{Q}_{11}(14.5)$ \\
\hline 15.5 & 15.5 & 16298.22 & ${ }^{\mathrm{Q}} \mathrm{R}_{12}+\mathrm{Q}_{11}(15.5)$ \\
\hline 16.5 & 16.5 & 16298.43 & ${ }^{\mathrm{Q}} \mathrm{R}_{12}+\mathrm{Q}_{11}(16.5)$ \\
\hline 17.5 & 17.5 & 16298.63 & ${ }^{\mathrm{Q}} \mathrm{R}_{12}+\mathrm{Q}_{11}(17.5)$ \\
\hline 18.5 & 18.5 & 16298.83 & ${ }^{\mathrm{Q}} \mathrm{R}_{12}+\mathrm{Q}_{11}(18.5)$ \\
\hline 19.5 & 19.5 & 16299.04 & ${ }^{\mathrm{Q}} \mathrm{R}_{12}+\mathrm{Q}_{11}(19.5)$ \\
\hline 20.5 & 20.5 & 16299.24 & ${ }^{\mathrm{Q}} \mathrm{R}_{12}+\mathrm{Q}_{11}(20.5)$ \\
\hline 21.5 & 21.5 & 16299.42 & ${ }^{Q_{R_{12}}+Q_{11}(21.5)}$ \\
\hline 22.5 & 22.5 & 16299.61 & ${ }^{\mathrm{Q}} \mathrm{R}_{12}+\mathrm{Q}_{11}(22.5)$ \\
\hline 23.5 & 23.5 & 16299.78 & ${ }^{\mathrm{Q}} \mathrm{R}_{12}+\mathrm{Q}_{11}(23.5)$ \\
\hline 24.5 & 24.5 & 16299.93 & ${ }^{\mathrm{Q} \mathrm{R}_{12}+\mathrm{Q}_{11}(24.5)}$ \\
\hline 25.5 & 25.5 & 16300.11 & ${ }^{\mathrm{Q}} \mathrm{R}_{12}+\mathrm{Q}_{11}(25.5)$ \\
\hline 26.5 & 26.5 & 16300.25 & ${ }^{\mathrm{Q}} \mathrm{R}_{12}+\mathrm{Q}_{11}(26.5)$ \\
\hline 6.5 & 5.5 & 16301.64 & $\mathrm{R}_{1}(5.5)$ \\
\hline 7.5 & 6.5 & 16302.85 & $\mathrm{R}_{1}(6.5)$ \\
\hline 8.5 & 7.5 & 16304.03 & $\mathrm{R}_{1}(7.5)$ \\
\hline
\end{tabular}




\begin{tabular}{|r|r|r|c|}
\hline 9.5 & 8.5 & 16305.21 & $\mathrm{R}_{1}(8.5)$ \\
\hline 10.5 & 9.5 & 16306.37 & $\mathrm{R}_{1}(9.5)$ \\
\hline 11.5 & 10.5 & 16307.55 & $\mathrm{R}_{1}(10.5)$ \\
\hline 12.5 & 11.5 & 16308.71 & $\mathrm{R}_{1}(11.5)$ \\
\hline 13.5 & 12.5 & 16309.88 & $\mathrm{R}_{1}(12.5)$ \\
\hline 14.5 & 13.5 & 16311.03 & $\mathrm{R}_{1}(13.5)$ \\
\hline 15.5 & 14.5 & 16312.15 & $\mathrm{R}_{1}(14.5)$ \\
\hline 16.5 & 15.5 & 16313.29 & $\mathrm{R}_{1}(15.5)$ \\
\hline 17.5 & 16.5 & 16314.4 & $\mathrm{R}_{1}(16.5)$ \\
\hline 18.5 & 17.5 & 16315.55 & $\mathrm{R}_{1}(17.5)$ \\
\hline 19.5 & 18.5 & 16316.67 & $\mathrm{R}_{1}(18.5)$ \\
\hline 20.5 & 19.5 & 16317.77 & $\mathrm{R}_{1}(19.5)$ \\
\hline 21.5 & 20.5 & 16318.87 & $\mathrm{R}_{1}(20.5)$ \\
\hline 22.5 & 21.5 & 16320 & $\mathrm{R}_{1}(21.5)$ \\
\hline
\end{tabular}




\section{CHAPTER VIII}

\section{FUTURE WORKS}

The current human knowledge of macro- and micro-cosmoses is mainly based on (special and general) relativity, quantum mechanics, and SM. In order to verify known physical laws, test their applicability and limitations, and, more importantly, discover new physical phenomena and physical laws, it is critical for physicists to go beyond SM. While collider-based high-energy physics has proven to be a powerful tool for the development of new physics, tabletop high-precision laser spectroscopy of atoms and molecules provides an alternative approach that can be equally potent and more costeffective. Unknown physics can be uncovered in high-precision laser spectroscopy studies because it affects the detectable energy level structure and dynamics of atoms and molecules. Thanks to advent and advance of a myriad of new technologies in recent years, including frequency stabilization techniques that provide unprecedented laser linewidth and frequency accuracy, optical frequency combs, atomic and molecular clocks, and laser-cooling and trapping of atoms and molecules, modern high-precision laser spectroscopy has reached the feV level energy precision, equivalent to high-energy physics at the PeV level.

One of the most important aspects of molecular spectroscopy for new physics is the detection of TRSV interactions, ${ }^{111}$ which leads to the electron's electric dipole moment (eEDM), astringent test to SM and other CP-violating models. Recently, Hutzler et al. ${ }^{112}$ 
proposed using laser-cooled linear and symmetry polyatomic molecules containing alkaline-earth or alkaline-earth-like metal atoms with an ionic, monovalent chemical bond for detection of TRSV. The metal center of these molecules is sensitive to a wide range of symmetry-violating physics. In particular, alkaline-earth(-like) monohydroxides (MOHs) have been chosen as first candidate molecules for the TRSV measurement for the following reasons.

(1) Searches for new physics with molecules typically involve measuring phases that accumulate as the molecule processes in electromagnetic fields. Long precession time or coherence time (as long as tens of seconds) is therefore needed for experimental sensitivity. In order to achieve long coherent time, molecules produced in supersonic beams or cryogenic buffer gas beams (CBGBs) need to be laser-cooled and trapped in a magneto-optical trap (MOT) to reach milliKelvin temperature level. Due to their unique chemical bonding, MOHs are amenable to laser cooling. In the ground $\left(\tilde{X}^{2} \Sigma^{+}\right)$and firstexcited $\left(\tilde{A}^{2} \Pi\right)$ electronic states of $\mathrm{MOH}$, the unpaired electron is localized on the $s$ and $p \pi$ orbitals of the valence shell of the alkaline-earth metal atom. Moreover, orbital hybridization induced by the bond pushes the M orbitals away from the bonding region. Therefore, the electronic motion of MOHs is largely localized on the alkaline-earth metal atom and mostly decoupled from the vibrational modes of the molecule. As a result, laser-cooling and trapping techniques similar to those for atoms can be applied to MOHs.

(2) Degenerate vibrational and rotational motions of these linear molecules provide energy-level doublets that enable full polarization and internal co-magnetometers, which allows for the reversal of the EDM interaction without changing any lab fields, and hence 
provide crucial robustness to systematic effects. For instance, vibrational states of the MO-H bending mode with opposite parties can be easily polarized in electric fields.

High-precision measurements of energy level structure of relevant states and transition intensities are a prerequisite of future laser cooling of $\mathrm{MOHs}$ and detection of TRSV. Especially important are the fundamental and overtone levels of the aforementioned $\mathrm{M}-\mathrm{O}-\mathrm{H}$ bending mode $\left(v_{2}\right)$. Being a degenerate vibrational mode, $v_{2}$ levels in orbitally non-degenerate $\Sigma$ electronic states are split to $v+1$ levels with $|l|=0,1 \ldots v$, where $v$ is the vibrational quantum number and $l$ is the vibrational angular momentum quantum number. The Coriolis interaction further splits the $+l$ and $-l$ levels. The population on the $\left(v_{1} v_{2}{ }_{2} v_{3}\right)=\left(01^{1} 0\right)$ level of the $\tilde{X}^{2} \Sigma^{+}$state will be used for the proposed TRSV measurement. In orbitally doubly degenerate electronic states ( $\Pi, \Delta$, etc.), $v_{2}$ vibrational levels are split into six spin-vibronic levels by the spin-orbit (SO) and the Renner-Teller (RT) effects. ${ }^{113}$ 


\section{High-precision laser-spectroscopy measurements of MOH molecules}

\subsection{Measurement of spin-rovibrational structure of the ground $\left(\widetilde{X}^{2} \Sigma^{+}\right)$ and the first-excited $\left(\widetilde{A}^{2} \Pi\right)$ electronic states of MOHs}

These two states are directly involved in both laser cooling and the TRSV measurement. Energy-level structure of the $\tilde{A}^{2} \Pi$ and $\tilde{X}^{2} \Sigma^{+}$states will be mapped out in $\tilde{A}^{2} \Pi \leftarrow \tilde{X}^{2} \Sigma^{+}$laser-induced fluorescence (LIF) and $\tilde{A}^{2} \Pi \rightarrow \tilde{X}^{2} \Sigma^{+}$dispersed fluorescence (DF) measurements, respectively, using an existing apparatus in the Liu Lab. A pulsed laser will be used for the LIF survey scan that provides the vibrational structure of the $\tilde{A}^{2} \Pi$ state, followed by high-resolution LIF scans using a continuous-wave (cw) laser to reveal the rotational and fine structure. Resolution and frequency precision of the LIF measurement is limited by the laser linewidth, whereas those of the DF measurement is limited by the resolution of the spectrograph to several wavenumbers. To determine the $\tilde{X}^{2} \Sigma^{+}$-state energy level structure with better resolution and frequency precision, a second “dump” laser (cw or pulsed) will be added to the LIF experiment to carry out a stimulated emission pumping (SEP) measurement, whose resolution and precision is determined mainly by the dump laser (Fig. 1a).

Moreover, we will design and develop a new high-sensitivity spectroscopic technique for the measurement of the $\tilde{X}^{2} \Sigma^{+}$-state energy level structure, namely, SEP-cavity ring-up (SEP-CRU) spectroscopy. In the SEP-CRU experiment, a cw laser will be used to excite (or "pump”) $\tilde{X}^{2} \Sigma^{+}$-state population to an excited energy level, e.g., the vibrational ground level of the $\tilde{A}^{2} \Pi$ state. Like in the SEP experiment, a second laser (cw or pulsed) will be used to stimulate (or "dump") the excited-state population to 
the ground state. A high-finesse Fabry-Pérot cavity will be constructed to trap the dump laser beam, and the leak-out photons will be detected (Fig. 1b). The decay of the dump laser transmission can be fit to determine the ring-down time $(\tau) . \tau$ increases when the dump laser frequency is in resonance with an $\tilde{A}^{2} \Pi \rightarrow \tilde{X}^{2} \Sigma^{+}$transition (Fig. 1c). 


\subsection{Detection of the $\widetilde{A^{\prime 2}} \Delta$ dark state of MOHs}

Dark states play a critical role in the laser cooling of atoms and molecules. Population loss due to relaxation to dark states determines the maximum averaged a number of scattering events an atom or molecule can experience. In MOHs, the dark electronic state relevant to laser-cooling is the $\widetilde{A}^{\prime 2} \Delta$ state that is in close proximity to the $\tilde{A}^{2} \Pi$ as well as $\tilde{B}^{2} \Sigma^{+}$states. Since it is “dark”, the $\widetilde{A}^{\prime 2} \Delta$ state doesn't fluoresce significantly and cannot be detected by LIF spectroscopy with a high signal-to-noise ratio (SNR).

However, it can be detected using direct-absorption techniques such as cavity ring-down (CRD) spectroscopy. In a proof-of-principle experiment, $\tilde{A}^{2} \Pi \leftarrow \tilde{X}^{2} \Sigma^{+}$ the transition of the YO molecule, one of the only three diatomic molecules that have been laser-cooled, was detected using a pulsed laser (Fig. 8.1d).

The pulsed-CRD spectroscopy has a sensitivity comparable to LIF spectroscopy. A cwCRD spectroscopy apparatus is under construction, which is expected to increase the SNR by two to three orders of magnitude.

\subsection{Measurement of FCFs}

Accurate determination of FCFs for both $\tilde{A}^{2} \Pi \leftarrow \tilde{X}^{2} \Sigma^{+}$and $\tilde{A}^{2} \Pi \rightarrow \tilde{X}^{2} \Sigma^{+}$ transitions is critical to the proposed project. The efficiency of laser cooling is determined by FCFs, among other factors. The $\tilde{A}^{2} \Pi \rightarrow \tilde{X}^{2} \Sigma^{+}$transition FCFs also determine the number of re-pumping lasers required in laser cooling. Furthermore, the efficiency of 
population transfer to the $\tilde{X}^{2} \Sigma^{+}\left(01^{1} 0\right)$ level, used for the TRSV measurement, is determined by FCFs for the $\tilde{A}^{2} \Pi\left(0 v_{2} 0\right) \leftarrow \tilde{X}^{2} \Sigma^{+}(000)$ and $\tilde{A}^{2} \Pi\left(0 v_{2} 0\right) \rightarrow \tilde{X}^{2} \Sigma^{+}\left(01^{1} 0\right)$ transitions. Measured FCFs will help choose the intermediate spin-ro-vibronic energy level for the most efficient population transfer to the $\tilde{X}^{2} \Sigma^{+}\left(01^{1} 0\right)$ level.

Traditionally, FCFs of the $\tilde{A}^{2} \Pi \leftarrow \tilde{X}^{2} \Sigma^{+}$and $\tilde{A}^{2} \Pi \rightarrow \tilde{X}^{2} \Sigma^{+}$transitions of MOHs are determined in LIF and DF measurements, respectively. However, the accuracy of the FCFs so determined is limited by the interference of the scattering of the excitation laser. In addition, when a pulsed excitation laser is used, the FC-favored transitions may easily be saturated, which leads to under-evaluated FCFs for these transitions. The aforementioned CRD and SEP-CRU techniques can be applied to measure FCFs of the $\tilde{A}^{2} \Pi \leftarrow \tilde{X}^{2} \Sigma^{+}$and $\tilde{A}^{2} \Pi \rightarrow \tilde{X}^{2} \Sigma^{+}$transitions, respectively. In both experiments, the transition intensity is measured as a change in $\tau$. Therefore, detected laser scattering, if any, does not affect the measured transition intensity. Because the inter-cavity field of the CRD or CRU laser is relatively weak, a saturation of transitions can be avoided. FCFs can, therefore, be determined in CRD and SEP-CRU experiments much more precisely than LIF/DF measurements. In the SEP-CRU experiment, the CRU signal can be normalized by the total detected fluorescence following excitation, which suppresses noise introduced by fluctuations in the concentration of molecules and the pump laser power. 
(a)

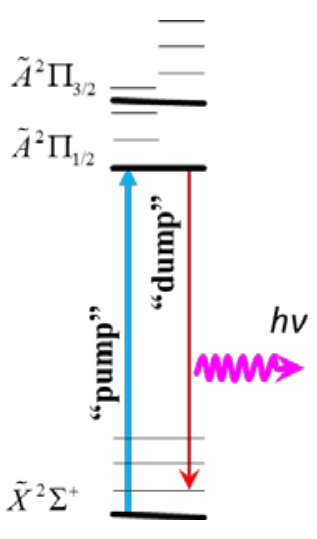

(b)
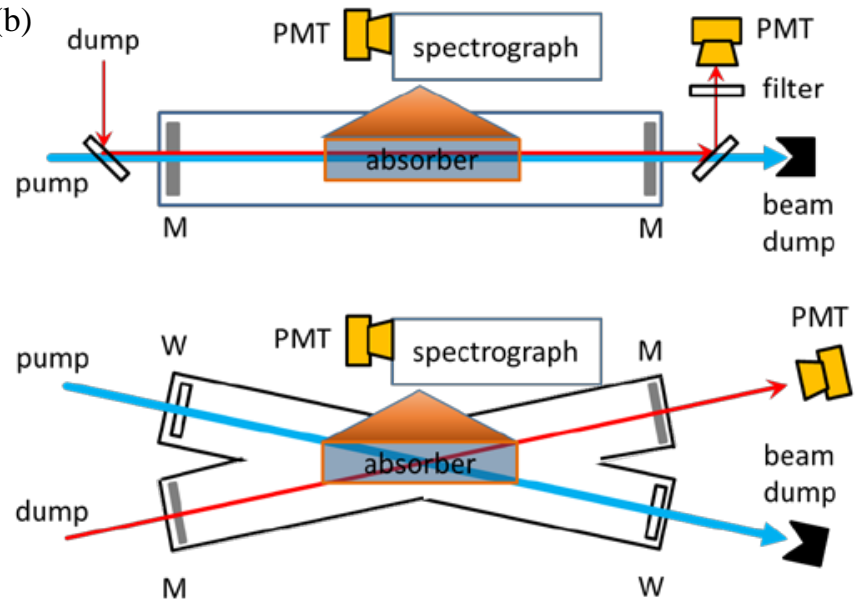

W

(c)
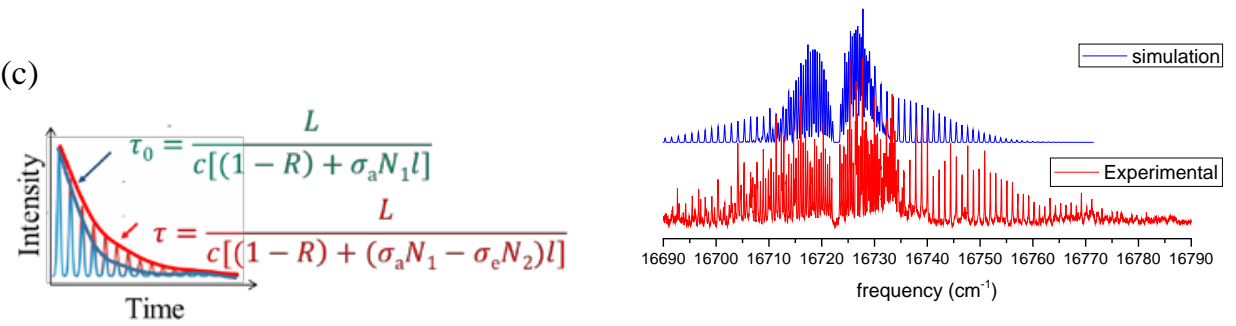

Figure 8.1 (a) Principle of SEP and SEP-CRU spectroscopy techniques. (b) Simplified experimental setups of SEP-CRU spectroscopy. When the pump and dump laser wavelengths are well separated, dichroic mirrors or (polarization) beam splitters are used to overlap them (top). When the wavelengths are close, a " $X$ "-shaped two-wavelength scheme is adopted (bottom). " $\mathrm{M}$ "=cavity ring-down mirror. "W"=window. PMT=photomultiplier tube. The spectrograph is used to select the wavelength window for fluorescence detection and to filter out laser scattering. (c) Ring-down curves without $\left(\tau_{0}\right)$ and with $(\tau)$ resonance between the dump laser frequency and an $\tilde{A}^{2} \Pi \rightarrow \tilde{X}^{2} \Sigma^{+}$transition. $c$ is the speed of light. $R$ is the reflectivity of ring-down mirrors. $\sigma_{\mathrm{a}}$ and $\sigma_{\mathrm{e}}$ are cross sections for absorption and emission, respective. $N_{1}$ and $N_{2}$ are populations

$$
\tilde{A}^{2} \Pi \leftarrow \tilde{X}^{2} \Sigma^{+}
$$




\section{REFERENCES}

1. Grubbs, R. H.; Tumas, W. J., Polymer synthesis and organotransition metal chemistry. Science. 1989, 243 (4893), 907-915.

2. Brazier, C.; Ellingboe, L.; Kinsey-Nielsen, S.; Bernath, P., Laser spectroscopy of alkaline earth monoalkoxide free radicals. J. Am. Chem. Soc. 1986, 108 (9), 2126-2132.

3. Paul, A. C.; Reza, M. A.; Liu, J., Dispersed-fluorescence spectroscopy of jetcooled calcium ethoxide radical $\left(\mathrm{CaOC}_{2} \mathrm{H}_{5}\right)$. J. Mol. Spectrosc. 2016, 330, 142-146.

4. Isaev, T. A.; Berger, R., Polyatomic candidates for cooling of molecules with lasers from simple theoretical concepts. Phys. Rev. Lett. 2016, 116 (6), 063006.

5. Shuman, E. S.; Barry, J. F.; DeMille, D. J., Laser cooling of a diatomic molecule. Nature. 2010, 467 (7317), 820.

6. Kozyryev, I.; Steimle, T. C.; Yu, P.; Nguyen, D.-T.; Doyle, J. M., Determination of $\mathrm{CaOH}$ and $\mathrm{CaOCH}_{3}$ vibrational branching ratios for direct laser cooling and trapping. New J. Phys. 2019, 21, 052002.

7. Yeo, M.; Hummon, M. T.; Collopy, A. L.; Yan, B.; Hemmerling, B.; Chae, E.; Doyle, J. M.; Ye, J., Rotational state microwave mixing for laser cooling of complex diatomic molecules. Phys. Rev. Lett. 2015, 114 (22), 223003.

8. Andrews, D. L.; Demidov, A. A., An introduction to laser spectroscopy. Springer Science \& Business Media: 2012. 
9. Ashfold, M.; Howe, J., Multiphoton spectroscopy of molecular species. Ann. Rev. Phys. Chem. 1994, 45 (1), 57-82.

10. Hirota, E., Plasma diagnosis by high-resolution spectroscopy of transient molecules. Pure App. Chem. 1998, 70 (6), 1145-1150.

11. Ross, I. G., High resolution electronic spectra of large polyatomic molecules. Adv. Chem. Pys. 1971, 20, 341.

12. Callow, J., Cellular and molecular recognition between higher plants and fungal pathogens. In Cellular interactions, Springer: 1984; pp 212-237.

13. Jamhawi, A.; Paul, Anam C.; Smith, Justin D.; Handa, Sachin.; Liu, J.; In Ultrafast Transient Absorption Spectroscopy of Polymer-Based Organophotoredox Catalysts Mimicking Transition-Metal Complexes, International Symposium on Molecular Spectroscopy: 2017.

14. Russell, D. K.; Davidson, I. M.; Ellis, A. M.; Mills, G. P.; Pennington, M.; Povey, I. M.; Raynor, J. B.; Saydam, S.; Workman, A. D., Mechanisms of Pyrolysis of Tricarbonylcyclopentadienylmanganese and Tricarbonyl (methylcyclopentadienyl) manganese. Organometalluics. 1995, 14 (8), 3717-3723.

15. Lapp, M., Flame temperatures from vibrational Raman scattering. In Laser Raman gas diagnostics, Springer: 1974; pp 107-145.

16. Goodings, J. M.; Patterson, P. M.; Hayhurst, A. N., Faraday Transactions, Massspectrometric study of the production and recombination of $\mathrm{BaOH}^{+}$ions and free electrons from barium added to flames of $\mathrm{H}_{2}+\mathrm{O}_{2}+$ Ar. J. Chem. Soc. 1995, 91 (15), 2257-2267.

17. Chapman, S., Notes on Atmospheric Sodium. Astrophys. J. 1939, 90, 309. 
18. Murad, E.; Swider, W.; Benson, S. W. J., Possible role for metals in stratospheric chlorine chemistry. Nature. 1981, 289 (5795), 273.

19. Tsuji, T., Molecular abundances in stellar atmospheres. II.Astronomy and Astrophys. 1973, 23, 411-431.

20. Ishii, K.; Hirano, T.; Nagashima, U.; Weis, B.; Yamashita, K., An ab initio prediction of the spectroscopic constants of MgNC-The first Mg-bearing molecule in space. Astrophys. J. 1993, 410, L43.

21. Ziurys, L. M.; Apponi, A.; Guélin, M.; Cernicharo, J., Detection of MgCN in IRC+ 10216: a new metal-bearing free radical. Astrophys. J 1995, 445, L47-L50.

22. Huber, K.-P., Molecular spectra and molecular structure: IV. Constants of diatomic molecules. Springer Science \& Business Media: 2013.

23. Bass, A. M.; Kuebler, N.; Nelson, L. J. T., Observation of FeO in absorption by flash heating and kinetic spectroscopy. J. Chem. Phys. 1964, 40 (10), 3121-3122.

24. Von Rosenberg Jr, C.; Wray, K.; Transfer, R., Shock tube studies on Fe (CO) $5^{+}$ $\mathrm{O}_{2}: 11 \mu \mathrm{FeO}$ emission and kinetics. J. Quant. Spectrosc. 1972, 12 (4), 531-547.

25. Rosen, B., Spectra of diatomic oxides by the method of exploded wire. Nature 1945, 156 (3967), 570.

26. Dhumwad, R.; Bass, A. M., A Low-Pressure Arc Source for the Emission Spectrum of the FeO Molecule. Applied Optics 1963, 2 (12), 1335-1335.

27. Herschel, J. F. W. J. E.; Edinburgh, E. S. T. o. T. R. S. o., XXXI. On the Absorption of Light by Coloured Media, and on the Colours of the Prismatic Spectrum exhibited by certain Flames; with an Account of a ready Mode of determining the absolute dispersive Power of any Medium, by direct experiment. 1823, 9 (2), 445-460. 
28. West, J.; Bradford Jr, R.; Eversole, J.; Jones, C., Flow system for the production of diatomic metal oxides and halides. Rev. Scient. Instru. 1975, 46 (2), 164-168.

29. Bernath, P. F., Gas-phase inorganic chemistry: monovalent derivatives of calcium and strontium. Science 1991, 254 (5032), 665-670.

30. Bernath, P.; Pinchemel, B.; Field, R. J. T., The hyperfine structure of the calcium monohalides. J. Chem. Phys. 1981, 74 (10), 5508-5515.

31. Trkula, M.; Harris, D. O.; Hilborn, R. C., Hollow-cathode sputtering source for the production of gas-phase metal atoms of the refractory elements. J. Chem. Phys. Lett. 1982, 93 (4), 345-349.

32. Schlachta, R.; Lask, G.; Tsay, S.; Bondybey, V., Pulsed discharge source of supersonically cooled transient species. Chem. Phys. 1991, 155 (2), 267-274.

33. Trkula, M.; Harris, Laser spectroscopy of the $1 \mathrm{~A}^{\prime \prime}-\tilde{\mathrm{X}} 1 \mathrm{~A}^{\prime}$ system of $\mathrm{CuOH}$ and CuOD. J. Chem. Phys. 1983, 79 (3), 1138-1144.

34. Steimle, T.; Tanimoto, M.; Namiki, K.; Saito, S. J. T., The millimeter wave spectrum of silver monoxide, AgO. J. Chem. Phys. 1998, 108 (18), 7616-7622.

35. Steimle, T.; Shirley, J.; Simard, B.; Vasseur, M.; Hackett, P., A laser spectroscopic study of gas-phase TiH. J. Chem. Phys. 1991, 95 (10), 7179-7182.

36. Steimle, T. C.; Shirley, J. E., The Stark effect in the $B^{3} \Pi-X^{3} \Delta$ band system of TiO. J. Chem. Phys. 1989, 91 (12), 8000-8002.

37. Clouthier, D.; Huang, G.; Merer, A.; Friedman-Hill, E., Hyperfine and spinorbit structure of the $4 \Delta$ i ground state of CoO. J. Chem. Phys. 1993, 99 (9), 6336-6344.

38. Kingston, C. T. Laser spectroscopy of some transition metal-containing free radicals. University of British Columbia, 2002. 
39. Heaven, M.; Miller, T. A.; Bondybey, V., Production and characterization of temperature-controlled free radicals in a free jet expansion. Chem. Phys. Lett. 1981, 84 (1), 1-5.

40. Hansen, P. E., Carbon—hydrogen spin—spin coupling constants. Prog. Nucl. Magn. Reson. 1981, 14 (4), 175-295.

41. Dunlop, J. R.; Karolczak, J.; Clouthier, D. J., Pyrolysis jet spectroscopy. Chem. Phys. Lett. 1988, 151 (4-5), 362-368.

42. Povey, I. M.; Bezant, A. J.; Corlett, G. K.; Ellis, A. M., Spectroscopic Investigation of Zinc-Containing Organometallic Radicals Prepared Using a Pulsed Electrical Discharge Nozzle. J. Phys. Chem. 1994, 98 (41), 10427-10431.

43. Pooley, S. J.; Ellis, A. M., Dispersed Fluorescence Spectroscopy of the ZnC2H5Free Radical. J. Mol. Spectrosc. 1997, 185 (1), 48-53.

44. Ellis, A. M.; Robles, E. S.; Miller, T. A., Electronic spectroscopy of the openshell van der Waals complexes $\mathrm{X}-\mathrm{CdCH}_{3}(\mathrm{X}=\mathrm{He}, \mathrm{Ne}, \mathrm{Ar}, \mathrm{Kr}$, and Xe). J. Phys. Chem., 1993, 97 (22), 5809-5819.

45. Dietz, T. G.; Duncan, M. A.; Powers, D. E.; Smalley, R. E., Laser production of supersonic metal cluster beams. J. Chem. Phys. 1981, 74 (11), 6511-6512.

46. Gole, J.; English, J.; Bondybey, V., Laser spectroscopy of cooled metal clusters: copper dimer. J. Chem. Phys. 1982, 86 (14), 2560-2563.

47. Whitham, C.; Soep, B.; Visticot, J. P.; Keller, A., Observation and spectroscopy of metallic free radicals produced by reactive collisions during a supersonic expansion. J. Chem. Phys. 1990, 93 (2), 991-1000. 
48. Namiki, K.-i. C.; Robinson, J. S.; Steimle, T. C., A spectroscopic study of $\mathrm{CaOCH} 3$ using the pump/probe microwave and the molecular beam/optical Stark techniques. J. Chem. Phys. 1998, 109 (13), 5283-5289.

49. Loock, H.-P.; Bérces, A.; Simard, B.; Linton, C., Laser spectroscopy of the $\tilde{\mathrm{A}}^{2} \Pi \leftarrow \tilde{\mathrm{X}}^{2} \Sigma+$ transition of ytterbium monoacetylide. J. Chem. Phys. 1997, 107 (8), 27202727.

50. Simard, B.; Masoni, C.; Hackett, P., First laboratory observation of niobium monosulphide in the gas phase. J. Chem. Phys. 1990, 92 (12), 7003-7011.

51. Beardah, M. S.; Ellis, A. M., Observation of several new electronic transitions of the SrOH free radical. J. Chem. Phys. 1999, 110 (23), 11244-11254.

52. Little, A. M.; Corlett, G. K.; Ellis, A. M., UV absorption of LiO in a supersonic jet. Chem. Phys. Lett. 1998, 286 (5-6), 439-445.

53. Born, M.; Oppenheimer, R., Zur quantentheorie der molekeln. Annalen der Physik 1927, 389 (20), 457-484.

54. Jahn, H. A.; Teller, E., Stability of polyatomic molecules in degenerate electronic states-I—Orbital degeneracy. Procee. Roy. Soc. A 1937, 161 (905), 220-235.

55. Jahn, H., Stability of polyatomic molecules in degenerate electronic states II-Spin degeneracy. Procee. Roy. Soc. A 1938, 164 (916), 117-131.

56. Paul, A. C.; Reza, M. A.; Liu, J. In Dispersed-Fluorescence Spectroscopy of JetCooled Calcium Ethoxide Radical $\left(\mathrm{CaOC}_{2} \mathrm{H}_{5}\right)$, 71st International Symposium on Molecular Spectroscopy, 2016. 
57. Paul, A. C.; Reza, M. A.; Shuvra, P. D.; Liu, J. In Laser-Induced Fluorescence and Dispersed-Fluorescence Spectroscopy of Jet-Cooled Calcium Monoalkoxide Radicals, 73rd International Symposium on Molecular Spectroscopy, 2018.

58. Hollas, J. M., Modern spectroscopy. John Wiley \& Sons: 2004.

59. Miller, T. A., Chemistry and chemical intermediates in supersonic free jet expansions. Science 1984, 223 (4636), 545-553.

60. Reza, M. A.; Paul, A. C.; Reilly, N. J.; Alam, J.; Liu, , Dispersed Fluorescence Spectroscopy of Jet-Cooled Isobutoxy and 2-Methyl-1-butoxy Radicals. J. Phys. Chem. A. 2016, 120 (34), 6761-6767.

61. Reza, M. A.; Paul, A. C.; Reilly, N. J.; Liu, J., Laser-Induced Fluorescence and Dispersed Fluorescence Spectroscopy of Jet-Cooled Isopentoxy Radicals. J. Phys. Chem.

\section{A. 2019.}

62. Telfah, H.; Reza, M. A.; Alam, J.; Paul, A. C.; Liu, J., Direct Observation of Tetrahydrofuranyl and Tetrahydropyranyl Peroxy Radicals via Cavity Ring-Down Spectroscopy. J. Phys. Chem. Lett. 2018, 9 (16), 4475-4480.

63. Reza, M. A.; Telfah, H.; Paul, A. C.; Alam, J.; Liu, J. In Cavity Ring-Down Spectroscopy of 1-, 2-and 3-METHYL Allyl Peroxy Radicals, 73rd International Symposium on Molecular Spectroscopy, 2018.

64. Telfah, H.; Reza, M. A.; Paul, A. C.; Liu, J. In Observation of theltilde a<--tilde X Electronic Transitions of Tetrahydropyranyl and Tetrahydrofuranyl Peroxy Radicals by Room-Temperature Cavity Ring-Down Spectroscopy, 73rd International Symposium on Molecular Spectroscopy, 2018. 
65. Paul, A. C.; Telfah, H.; Hu, X.; Liu, J. In Cavity Ring-Down Spectroscopy of Jet-Cooled YO Molecules, 74th International Symposium on Molecular Spectroscopy, 2019.

66. Brazier, C.; Bernath, P., Observation of gas phase organometallic free radicals: Monomethyl derivatives of calcium and strontium. J. Chem. Phys. 1987, 86 (11), 59185922.

67. Brazier, C.; Bernath, P., The $\mathrm{A}^{-2} \mathrm{E}-\mathrm{X}^{2} \mathrm{~A}_{1}$ transition of monomethyl calcium: A rotational analysis. J. Chem. Phys. 1989, 91 (8), 4548-4554.

68. Kozyryev, I.; Baum, L.; Matsuda, K.; Doyle, J. M., Proposal for laser cooling of complex polyatomic molecules. Chem. Phys. Chem. 2016, 17 (22), 3641-3648.

69. Di Rosa, M., Concept, candidates, and supporting hyperfine-resolved measurements of rotational lines in the AX $(0,0)$ band of CaH. eur. Phys. J. D. 2004, 31, 395.

70. Stuhl, B. K.; Sawyer, B. C.; Wang, D.; Ye, J., Magneto-optical trap for polar molecules. Phys. Rev. Lett. 2008, 101 (24), 243002.

71. Crozet, P.; Martin, F.; Ross, A.; Linton, C.; Dick, M.; Adam, A. J., The A ${ }^{2} E-$ $\mathrm{X}^{2} \mathrm{~A}_{1}$ System of $\mathrm{CaOCH}_{3}$. J. Mol. Spectrosc. 2002, 213 (1), 28-34.

72. Kozyryev, I.; Steimle, T. C.; Yu, P.; Nguyen, D.-T.; Doyle, J. M., Determination of $\mathrm{CaOH}$ and $\mathrm{CaOCH}_{3}$ vibrational branching ratios for direct laser cooling and trapping. New. J. Phys. 2019, 21 (5), 052002.

73. Crozet, P.; Ross, A.; Linton, C.; Adam, A.; Hopkins, W.; Le Roy, R., Geometry of the CaOCH3 radical from isotope effects in the $\mathrm{A}^{2} \mathrm{E}-\mathrm{X}^{2} \mathrm{~A}_{1}$ transition. J. Mol. Spectrosc. 2005, 229 (2), 224-230. 
74. Abtew, T. A.; Sun, Y.; Shih, B.-C.; Dev, P.; Zhang, S.; Zhang, P., Dynamic Jahn-Teller effect in the NV- center in diamond. Phys. Review. Lett. 2011, 107 (14), 146403.

75. Coxon, J. A.; Li, M.; Presunka, P. I., Laser spectroscopy of the (010) $2 \Sigma(+), 2 \Sigma$ $(-)-(000) 2 \Sigma+$ parallel bands in the $\tilde{\mathrm{A}}^{2} \Pi-\mathrm{X}^{2} \Sigma^{+}$system of CaOH. J. Mol. Spectrosc. 1994, 164 (1), 118-128.

76. Kozyryev, I.; Hutzler, N. R., Precision measurement of time-reversal symmetry violation with laser-cooled polyatomic molecules. Phys. Rev. Lett. 2017, 119 (13), 133002.

77. Powers, D.; Hopkins, J.; Smalley, R., Laser production of jet-cooled radicals. methoxy and methoxy-argon. J. Phys. Chem. 1981, 85 (19), 2711-2713.

78. Reza, M. A.; Telfah, H.; Xu, R.; Liu, J., Room-Temperature Cavity Ring-Down Spectroscopy of Methylallyl Peroxy Radicals. J. Phys. Chem. A. 2019, 123 (16), 35103517.

79. Yalin, A.; Zare, R., Effect of laser lineshape on the quantitative analysis of cavity ring-down signals. Laser Phys. Lawrence 2002, 12 (8), 1065-1072.

80. Frisch, M.; Trucks, G.; Schlegel, H. B.; Scuseria, G.; Robb, M.; Cheeseman, J.; Scalmani, G.; Barone, V.; Mennucci, B.; Petersson, G. J. I., Wallingford, CT, Gaussian 09, revision a. 02, gaussian. 2009, 200, 28.

81. Stanton, J. F., On the vibronic level structure in the NO 3 radical. I. The ground electronic state. J. Chem. Phys. 2007, 126 (13), 134309. 
82. Ichino, T.; Wren, S. W.; Vogelhuber, K. M.; Gianola, A. J.; Lineberger, W. C.; Stanton, J. F. J, The vibronic level structure of the cyclopentadienyl radical. J. Chem. Phys. 2008, 129 (8), 084310.

83. Weichman, M. L.; Cheng, L.; Kim, J. B.; Stanton, J. F.; Neumark, D. M., Lowlying vibronic level structure of the ground state of the methoxy radical: Slow electron velocity-map imaging (SEVI) spectra and Köppel-Domcke-Cederbaum (KDC) vibronic Hamiltonian calculations. J. Chem. Phys. 2017, 146 (22), 224309.

84. Mozhayskiy, V.; Krylov, A., ezSpectrum, version 3.0; see http://iopenshell. usc. edu/downloads.

85. Ichino, T.; Gauss, J.; Stanton, J. F., Quasidiabatic states described by coupledcluster theory. J. Chem. Phys. 2009, 130 (17), 174105.

86. Barckholtz, T. A.; Miller, T. A., Quantitative insights about molecules exhibiting Jahn-Teller and related effects. Int. Rev. Phys. Chem. 1998, 17 (4), 435-524.

87. Codd, T. J. Spectroscopic Studies of the $\mathrm{A}^{2} \mathrm{E}$ "State of $\mathrm{NO}_{3}$. The Ohio State University, 2014.

88. Müller, C. W.; Newby, J. J.; Liu, C.-P.; Rodrigo, C. P.; Zwier, T. S., Duschinsky mixing between four non-totally symmetric normal coordinates in the S 1-S 0 vibronic structure of (E)-phenylvinylacetylene: a quantitative analysis. Phys. Chem. Chem. Phys. 2010, 12 (10), 2331-2343.

89. Oka, T., Vibration-Rotation Interaction in Symmetric-Top Molecules and the Splitting between $\mathrm{A}^{1}$ and $\mathrm{A}^{2}$ Levels. J. Chem. Phys. 1967, 47 (12), 5410-5426.

90. Gaubatz, U.; Rudecki, P.; Schiemann, S.; Bergmann, K., Population transfer between molecular vibrational levels by stimulated Raman scattering with partially 
overlapping laser fields. A new concept and experimental results. J. Chem. Phys. 1990, 92 (9), 5363-5376.

91. Vitanov, N. V.; Rangelov, A. A.; Shore, B. W.; Bergmann, K., Stimulated Raman adiabatic passage in physics, chemistry, and beyond. Rev. Modern Phys.2017, 89 (1), 015006.

92. Salzberg, A. P.; Applegate, B. E.; Miller, T. A., Rovibronic Spectroscopy of

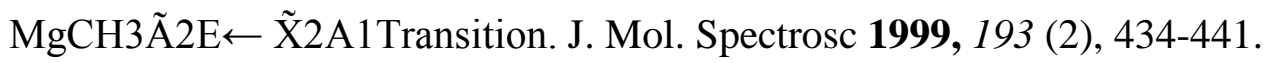

93. Sheridan, P.; Dick, M.; Wang, J.-G.; Bernath, P., High-Resolution Spectroscopic Investigation of the $\tilde{\mathrm{B}}^{2} \mathrm{~A}_{1}-\tilde{\mathrm{X}}^{2} \mathrm{~A}_{1}$ Transitions of $\mathrm{CaCH}_{3}$ and $\mathrm{SrCH}_{3}$. j. Phys. Chem. A. 2005, 109 (46), 10547-10553.

94. Forthomme, D.; Linton, C.; Read, A.; Tokaryk, D.; Adam, A.; Downie, L.; Granger, A.; Hopkins, W. J., Unravelling the visible spectrum of strontium monomethoxide. J. Mol. Spectrosc. 2011, 270 (2), 108-115.

95. Ham, F. S., Dynamical Jahn-Teller effect in paramagnetic resonance spectra: orbital reduction factors and partial quenching of spin-orbit interaction. Phys. Rev. 1965, 138 (6A), A1727.

96. Liu, J.; Melnik, D.; Miller, T. A., Rotationally resolved $\tilde{B} \leftarrow \tilde{X}$ electronic spectra of the isopropoxy radical: A comparative study. J. Chem. Phys. 2013, 139 (9), 094308.

97. Liu, J.; Miller, T. A., Jet-cooled laser-induced fluorescence spectroscopy of cyclohexoxy: Rotational and fine structure of molecules in nearly degenerate electronic states. J. Phys. Chem. A. 2014, 118 (51), 11871-11890. 
98. Bopegedera, A.; Fernando, W.; Bernath, P., Gas-phase inorganic chemistry: laser spectroscopy of calcium and strontium monopyrrolate molecules. J. Phys. Chem. 1990, 94 (11), 4476-4479.

99. Ortiz, J., Ground and excited states of $\mathrm{CaCH}_{3}, \mathrm{CaNH}_{2}, \mathrm{CaOH}$, and $\mathrm{CaF}$ through electron propagator calculations. J. Chem. Phys. 1990, 92 (11), 6728-6731.

100. Mozhayskiy, V.; Krylov, A., ezSpectrum, version 3.0. Los Angeles: University of Southern California. http://iopenshell. usc. edu ...: 2008.

101. Sharma, K.; Miller, T. A.; Paul, A. C.; Liu, J. In Analysis of Pseudo-Jahn Effect in Metal Alkoxides, 74th International Symposium on Molecular Spectroscopy, 2019. 102. Paul, A. C.; Sharma, K.; Reza, M. A.; Telfah, H.; Miller, T. A.; Liu, J., Laserinduced fluorescence and dispersed-fluorescence spectroscopy of the $\tilde{\mathrm{A}}^{2} \mathrm{E}-\tilde{\mathrm{X}}^{2} \mathrm{~A}_{1}$ transition of jet-cooled calcium methoxide $\left(\mathrm{CaOCH}_{3}\right)$ radicals. J. Chem. Phys. 2019, 151 (13), 134303.

103. Meggers, W.; Wheeler, J., The band spectra of Scandium, yttrium, and lanthanum monoxides, Bureau of Standards. J. Res.1931, 6, 239-275.

104. Johnson, L.; Johnson, R. J., Containing Papers of a Mathematical; Character, P., The band spectrum of yttrium oxide (YO). With a note on the $\mathrm{ScO}$ and $\mathrm{LaO}$ Systems. Proced. Roy. Sco. 1931, 133 (821), 207-219.

105. Linton, C. J., Photoluminescence of the $A^{2} \Pi-X^{2} \Sigma^{+}$system of the yttrium oxide molecule. J. Mol. Spectrosc. 1978, 69 (3), 351-364.

106. Uhler, U.; Akerlind, L. J., Rotational Analysis of The Blue-Green and Orange SystemsS of Yttrium Oxide. Arkiv. Fysik. 1961, 19. 
107. Hummon, M. T.; Yeo, M.; Stuhl, B. K.; Collopy, A. L.; Xia, Y.; Ye, J., 2D magneto-optical trapping of diatomic molecules. Phys. Rev. Lett. 2013, 110 (14), 143001.

108. Leung, J.-H.; Ma, T.; Cheung, A. J., Cavity ring down absorption spectroscopy of the $\mathrm{B}^{2} \Sigma^{+}-\mathrm{X}^{2} \Sigma^{+}$transition of YO. J. Mol. Spectrosc. 2005, 229 (1), 108-114.

109. Wu, S.; Dupré, P.; Miller, T. A., High-resolution IR cavity ring-down spectroscopy of jet-cooled free radicals and other species. Phys. Chem. Chem. Phys.2006, 8 (14), 1682-1689.

110. Bernard, A.; Bacis, R.; Luc, P., Fourier Transform Spectroscopy: Extensive Analysis of the $\mathrm{A}^{2} \mathrm{II}^{-} \rightarrow \mathrm{X}^{2} \mathrm{E}^{+}$and $\mathrm{B}^{2} \mathrm{E}^{+} \rightarrow \mathrm{X}^{2} \mathrm{E}^{+}$Systems of Yttrium Oxide. Astrophys. J. 1979, 227, 338-348.

111. Steimle, T.; Ubachs, W., Introduction to the Special issue Spectroscopic Tests of Fundamental Physics. Journal of Molecular Spectroscopy 2014, 300, 1-2.

112. Kozyryev, I.; Hutzler, N. R., Precision Measurement of Time-Reversal Symmetry Violation with Laser-Cooled Polyatomic Molecules. Phys. Rev. Lett. 2017, 119 (13), 133002.

113. Brown, J. M., The Renner-Teller effect: The effective Hamiltonian approach. In Computational molecular spectroscopy, Jensen, P.; Bunker, P. R., Eds. John Wiley \& Sons: 2000. 


\section{Appendices}

\section{A: Arduino Code for gear motor control of forward, backward and spinning motion}

The circuit diagram in figure 2.4 has represented an Arduino-Uno microcontroller along with an L298 dual H-Bridge motor control module. The microcontroller can be programmed in simple Arduino language which is merely a set of C/C++ functions and used to control the L298 H- Bridge motor control module. For our setup, we are using only one channel of the L298 dual H-Bridge. The DC gear motor is connected to the motor B lead out of the L298 module. The positive of DC power supply is connected to the $12 \mathrm{~V}$ input of L298 (figure 2.4) and its negative to the ground of L298. This ground is further connected to the ground pin of Arduino Uno. The PWM of L298 is connected to Pin 9 of Arduino Uno. For the direction control, two enable motor pin (IN3 and IN4) of L298 is connected to the two drives pin (Pin7 and Pin8) of Uno. A High signal and a LOW signal are used to control the direction of the motor. For example, a high to IN3 and a low to IN4 will cause the motor to turn in one direction, whereas a LOW signal to IN3 and a HIGH signal to IN4 will cause it to turn in the other direction. Sample code has been written to drive the motor (see below) 


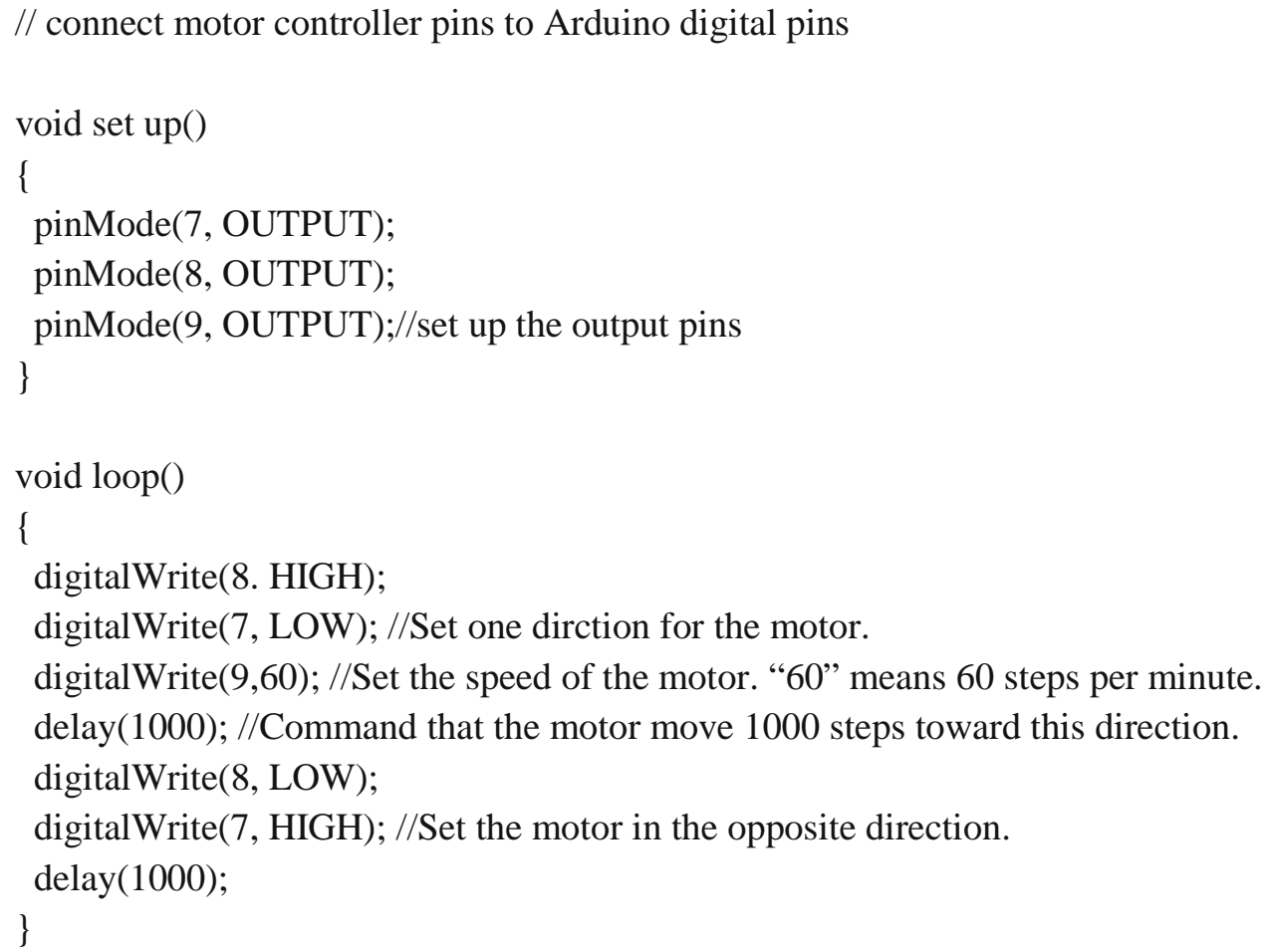

In this new version of the motorized stage, no feedback from the motor is needed to change the direction of the motor. Instead, the time duration was adjusted for the full use of the sample rod. No micro-switches are needed for changing the direction. 


\section{B: Python code for the dispersed-fluorescence (DF) spectra plot}

In our DF experiment, we are taking a scan frame by frame. For example, when we pumped a LIF band having a central wavelength (peak maximum) 630.3152 $\mathrm{nm}$, then in DF experiment we are scanning our spectrograph about $80 \mathrm{~nm}$ to the "red"' region which requires scanning at least eight frames. In this way, if we have 8 bands then we were taking at least 64 frames. Plotting them using origin software and patching together is time-consuming. We developed our own code in python so that plotting, patching, and smoothing can be done easily. Below is the code:

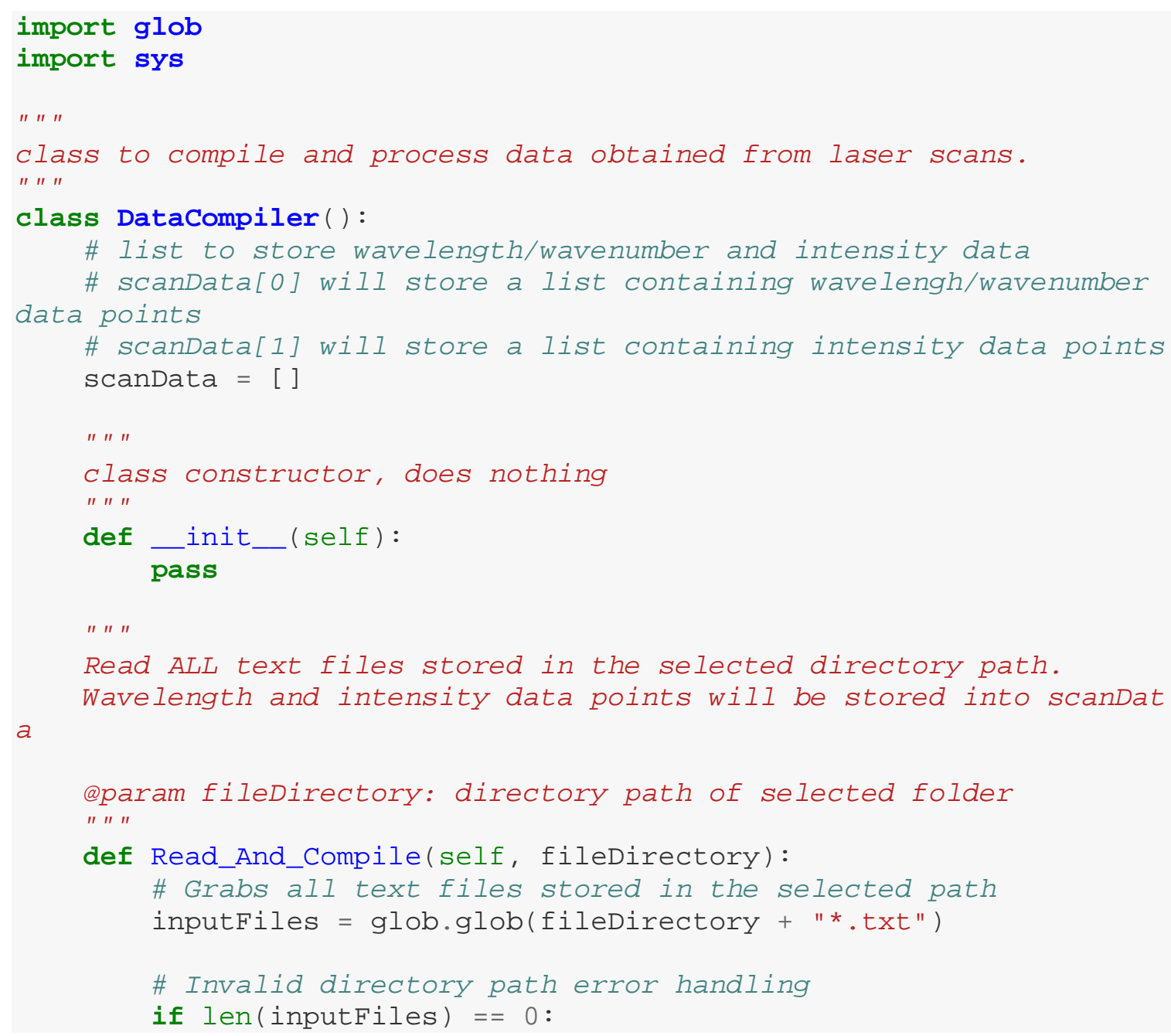


sys.exit("No text files found in this directory, check i $f$ the directory path is entered correctly")

\# Read and store data points into scanData

\# Note that wavelength are converted to float type while int ensity remains as str type

waveLength $=[]$

intensity $=[]$

for fileName in inputFiles:

with open(fileName, ' $r$ ') as $f d$ :

line $=\mathrm{fd}$.readline ()

while line:

lineData $=$ line.split ()

waveLength . append(float (lineData [๑]))

intensity . append (lineData[2])

line $=\mathrm{fd}$.readline ()

DataCompiler.scanData $=[$ waveLength, intensity $]$

" " " "

Convert wavelength to wavenumber

"I" "

def Nm_To_Wave_Number(self):

for $i$ in range(len(DataCompiler.scanData[○])):

anData $[\odot][i]$ DataCompiler.scanData[0][i] = $10000000 /$ DataCompiler.sc

" " " "

Modify wavenumber points by subtrating central frequency from $A L$ L data points

def X_Axis_Modification(self):

\#Prompt the user for central frequency number in $\mathrm{nm}$, needs $t$ o be greater than $\odot$

while True:

try:

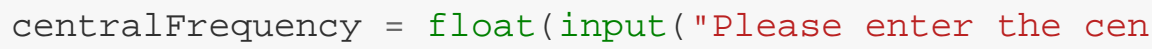
tral frequency in $\mathrm{nm}:$ ")) if centralFrequency $<=0$ :

๑") print("\nCentral Frequency must be greater than

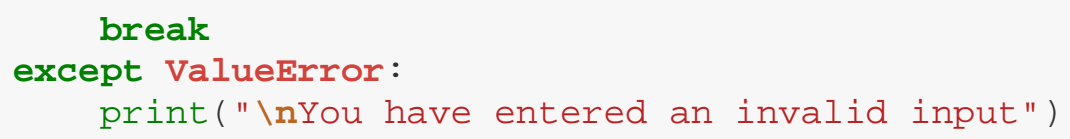

Save the data into a new text file named Compiled_Data.txt

@param fileDirectory: directory path selected to save the file, same as the input files 


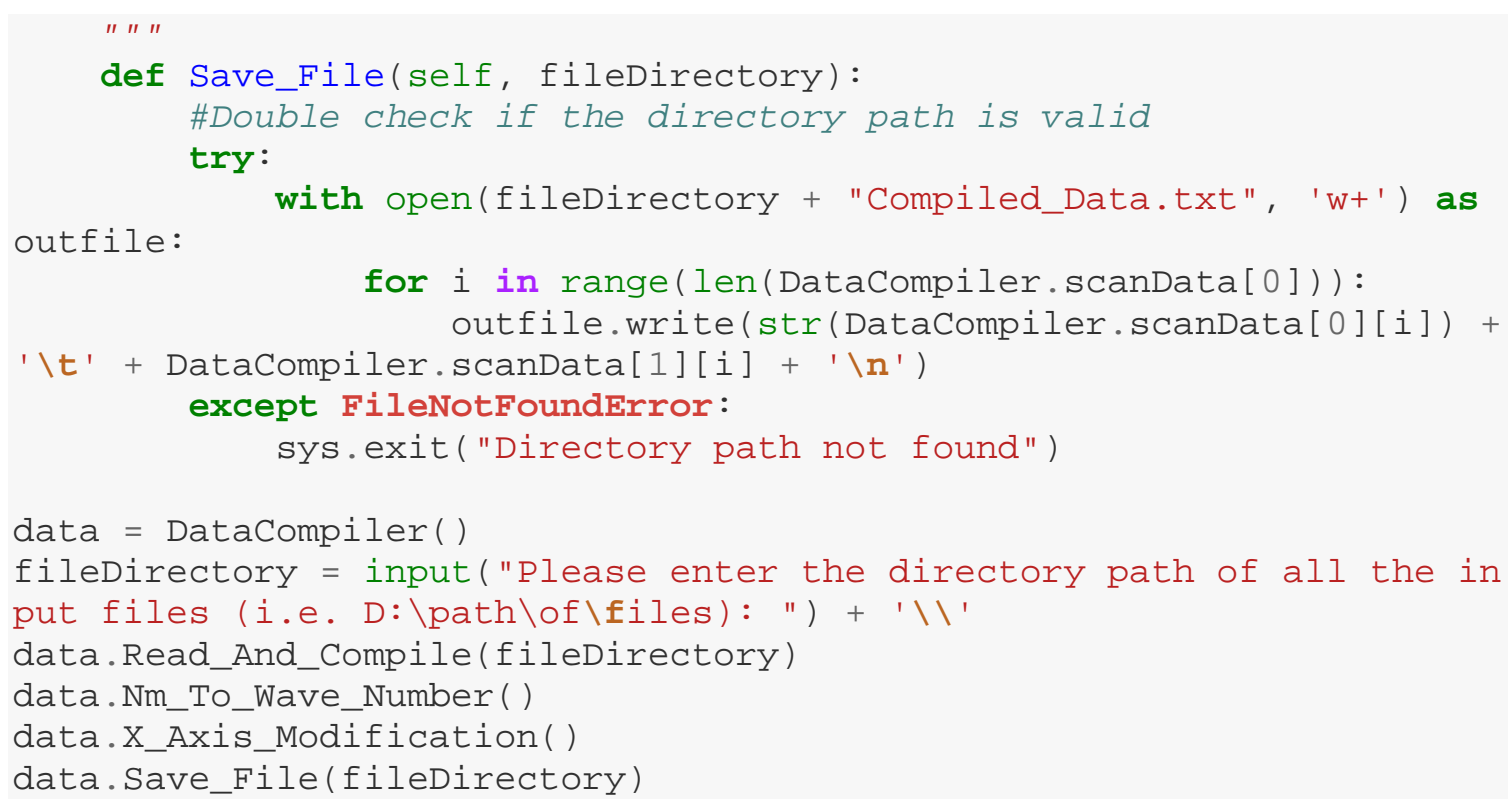




\section{C: Python code for mass weighted coordinate's to dimensionless}

\section{coordinate's conversion}

We did a couple of cluster calculations using Cfour program. By default, the program is generating log files with mass weighted coordinates. To generate a simulated spectrum we use ezSpectrum and ezSpectrum cannot deal with mass-weighted coordinates. Below is the python code which we used to convert mass-weighted to dimensionless coordinates.

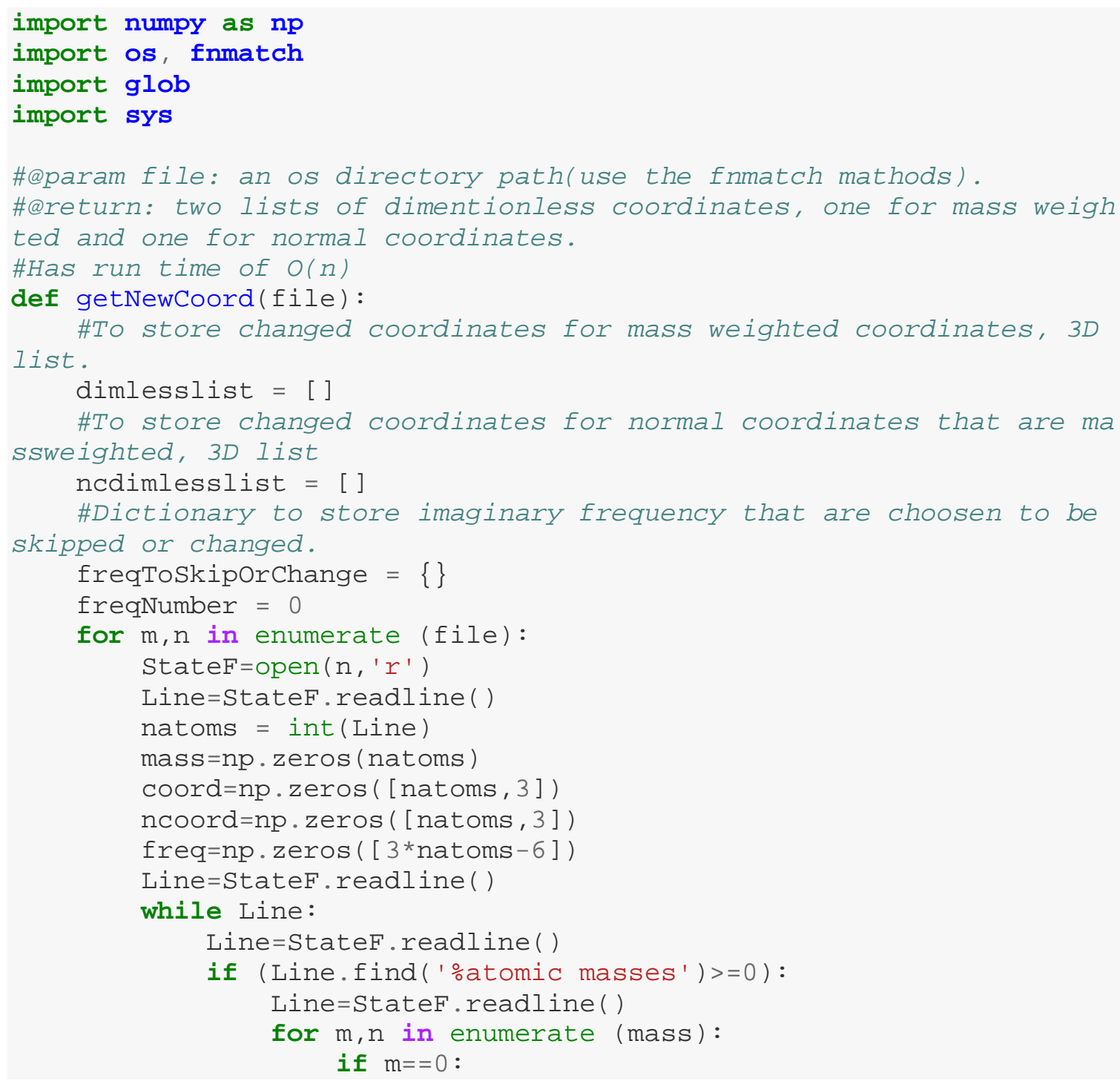




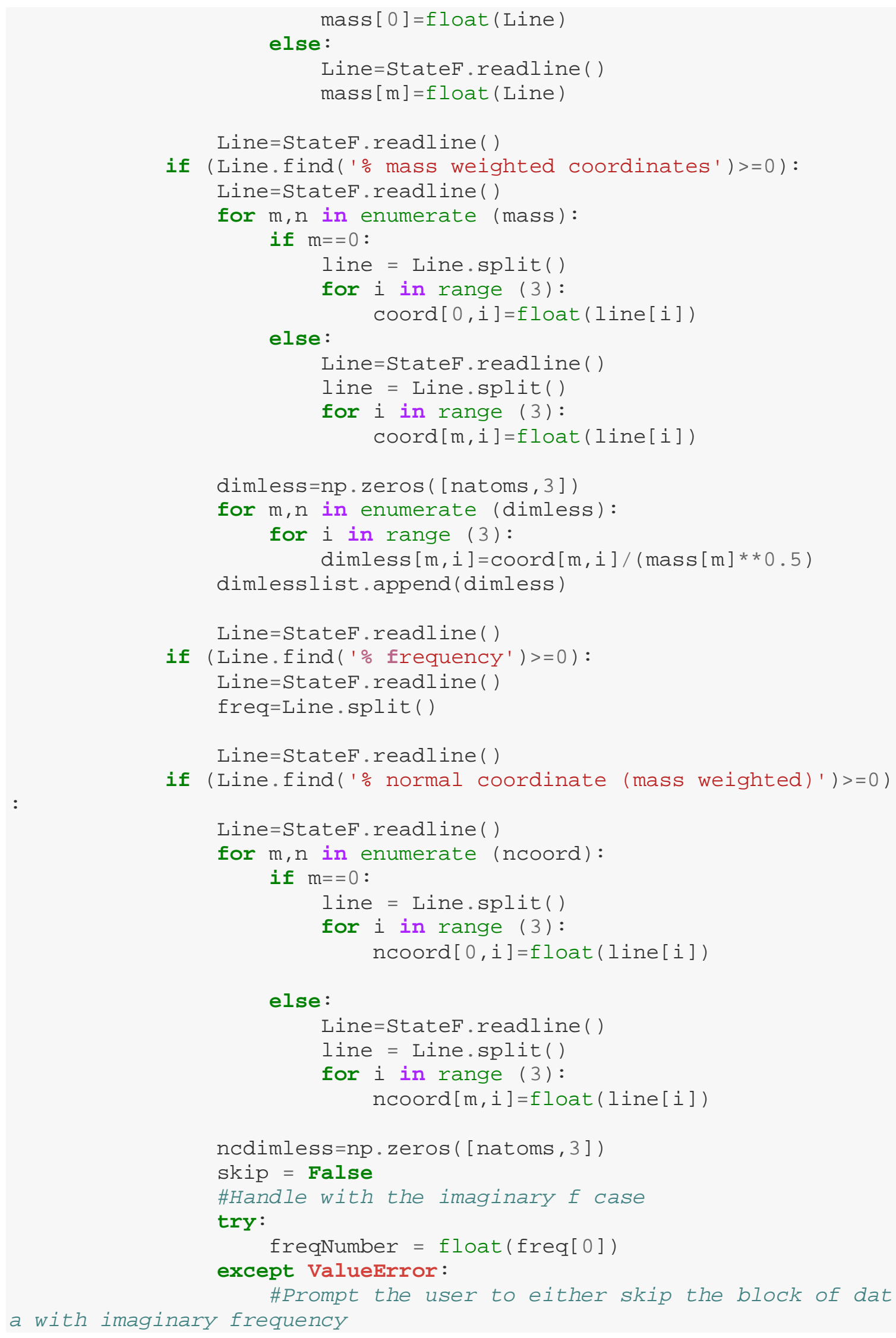


\#or enter a new valid number

while (True):

yorn = input("An imaginary frequency of \%s $\mathrm{w}$

as encountered, skip the data? \nEnter y or $n:$ " $\%$ freq[०])

if $($ yorn. lower ()$==$ ' $y$ ' or yorn. lower $==$ ' $n$ ' ) :

$s$ below to be executed, you can also use if conditions to achive the same thing...

valid freq: "))

qNumber

\section{else:}

\section{break}

print("Please enter y or $\mathrm{n} "$ )

if (yorn.lower ( ) == ' $y$ '):

freqToskiporChange $[$ freq $[\odot]]=$ None

skip = True

\section{continue}

skip = False

while True:

try:

newfreqNumber $=$ float $($ input $($ "Enter a freqToskiporChange $[$ freq $[\odot]]=$ newfre

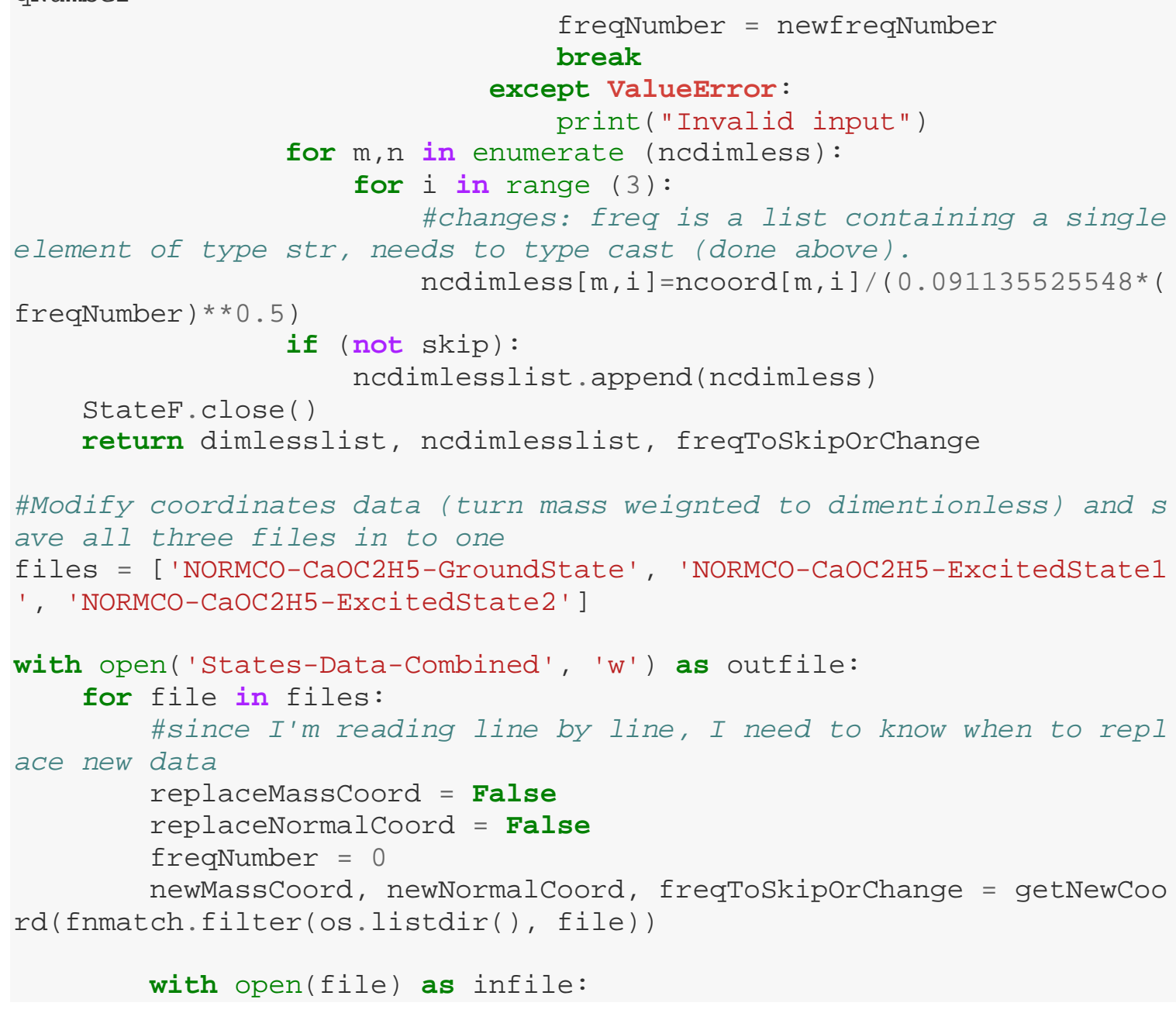




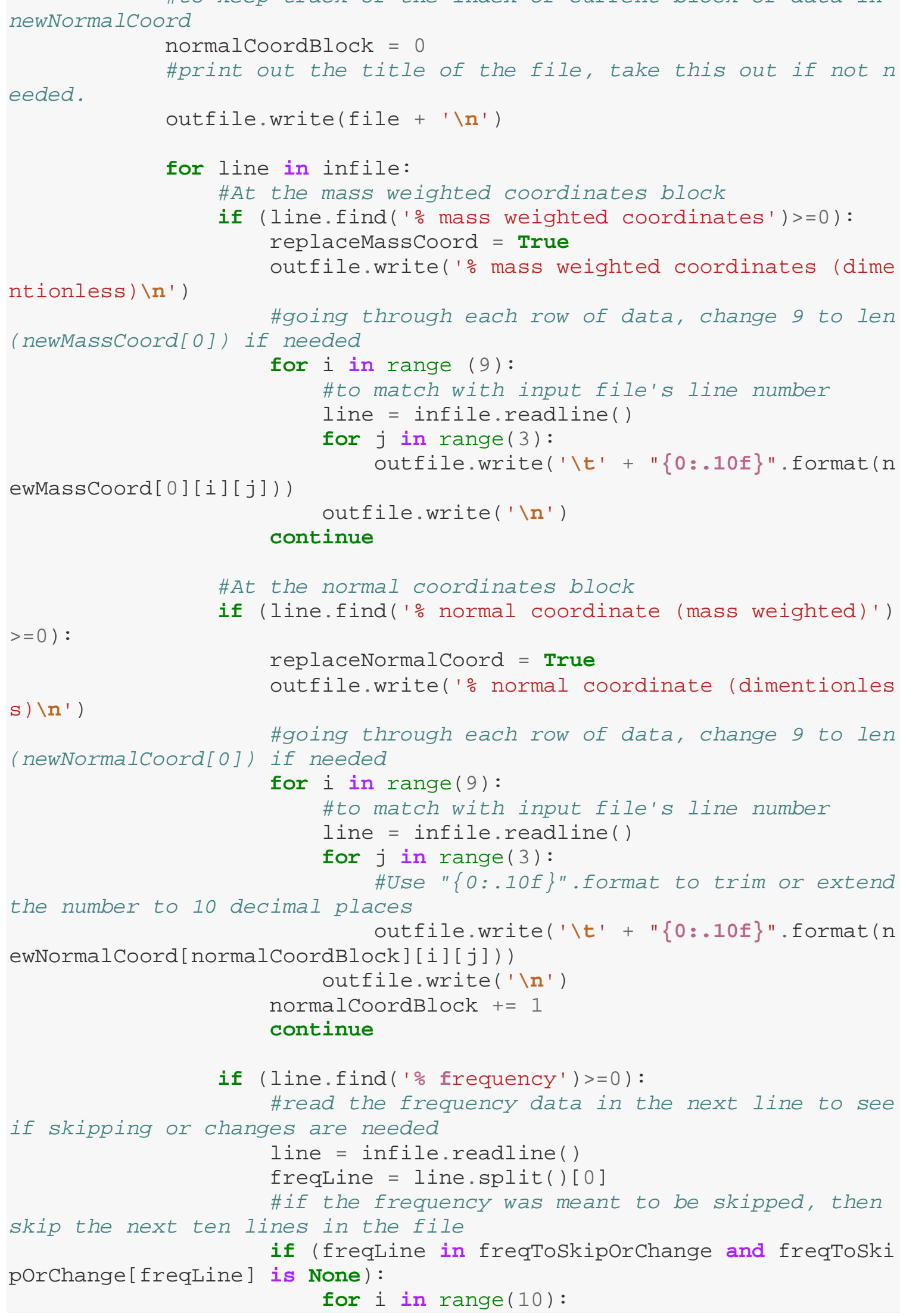




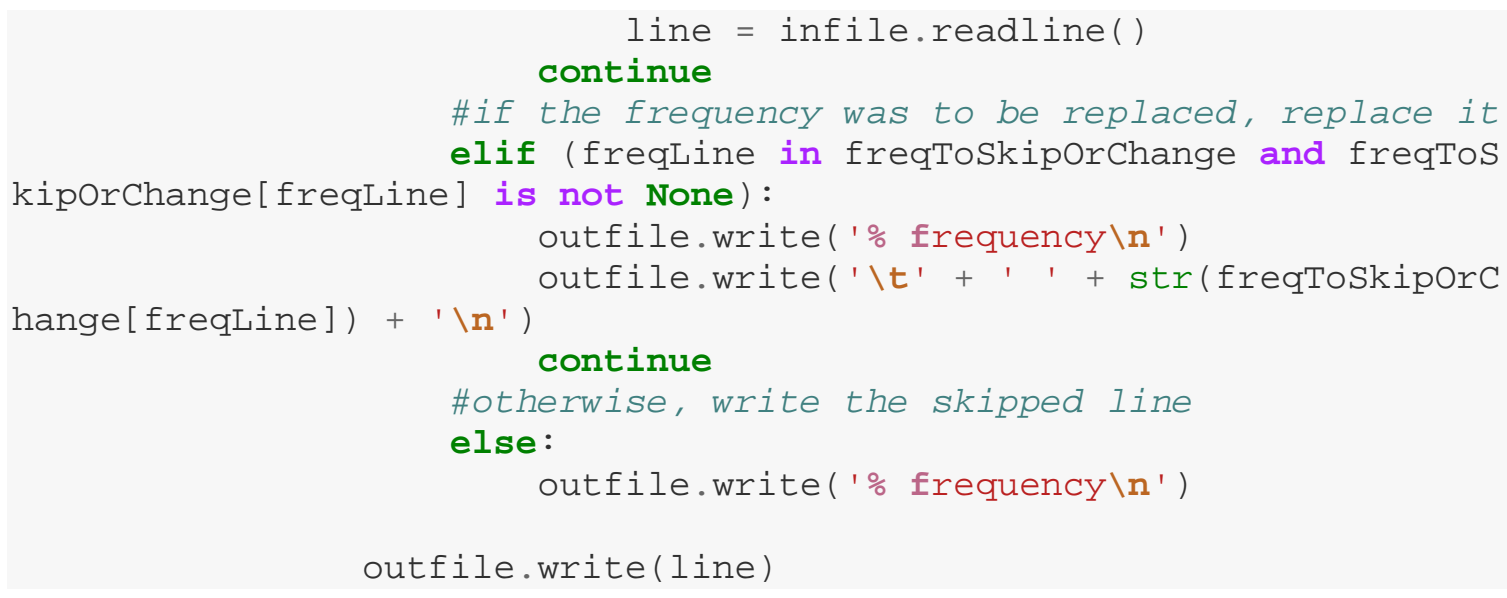




\section{D: Cartesian coordinates to Z-matrix conversion}

As we used to different software for ab-initio calculations that's why

sometimes we had to use either cartesian coordinates or Z-matrix based on the easiness

of the reading of log file. Below is the python code we used:

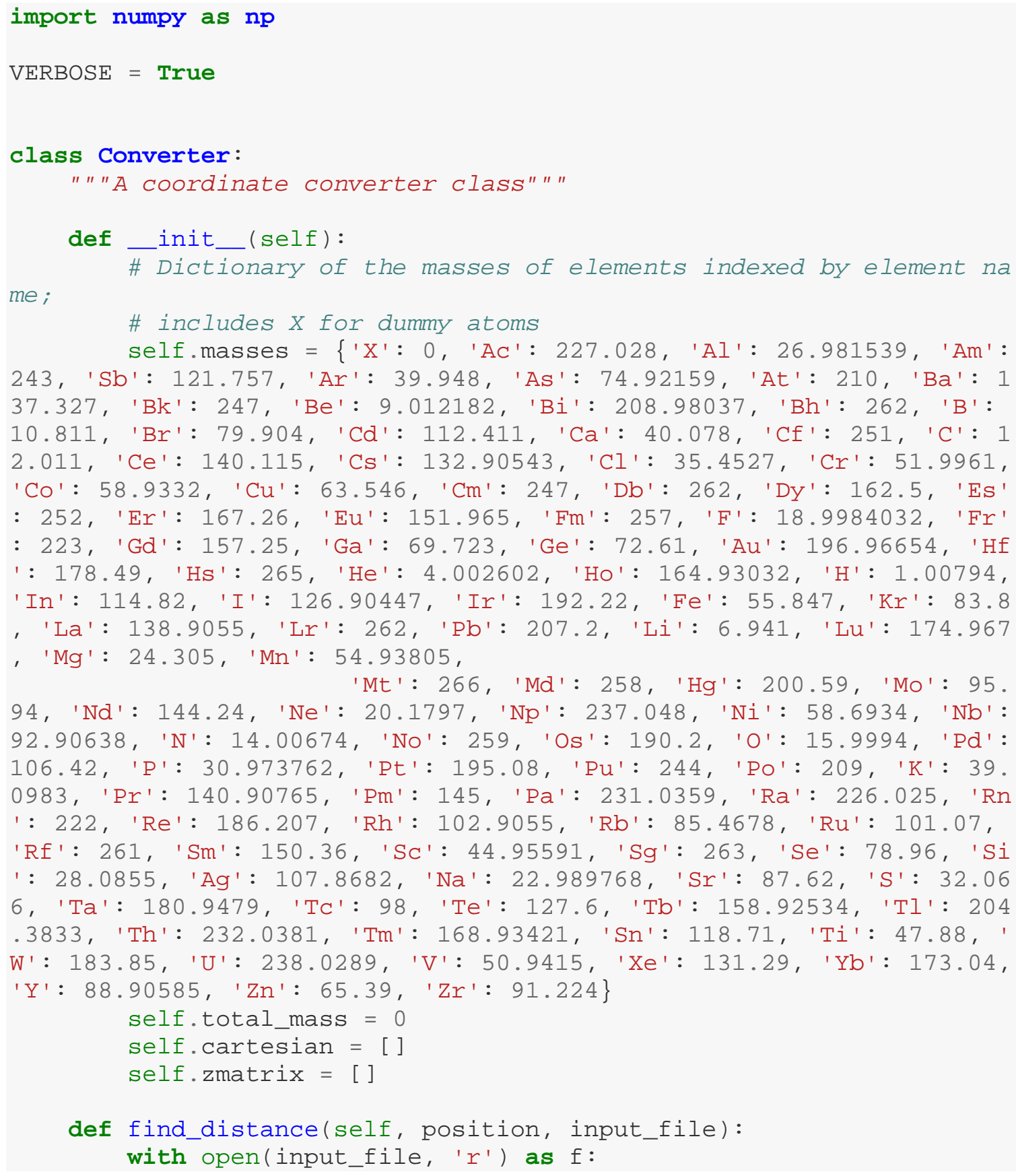




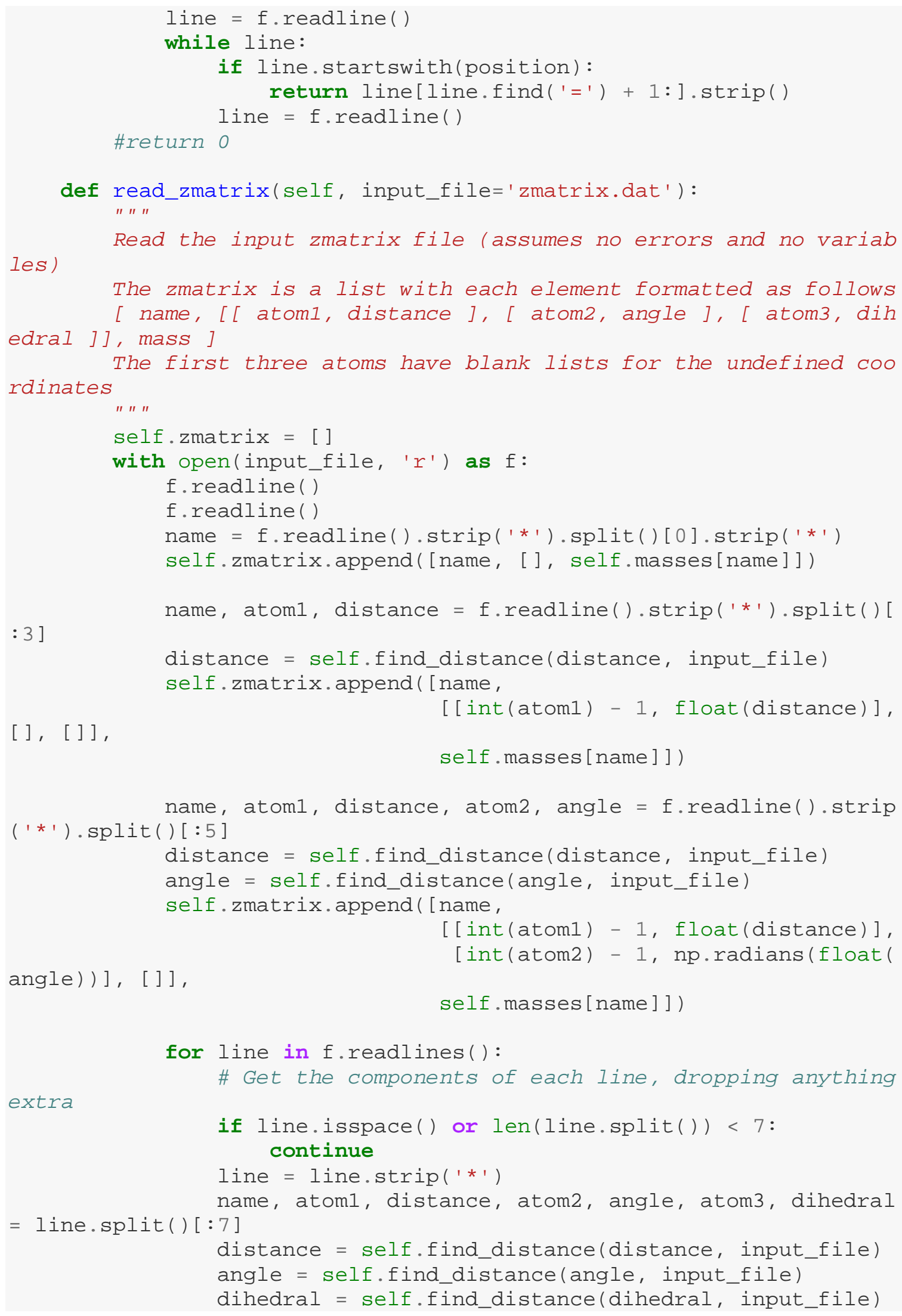




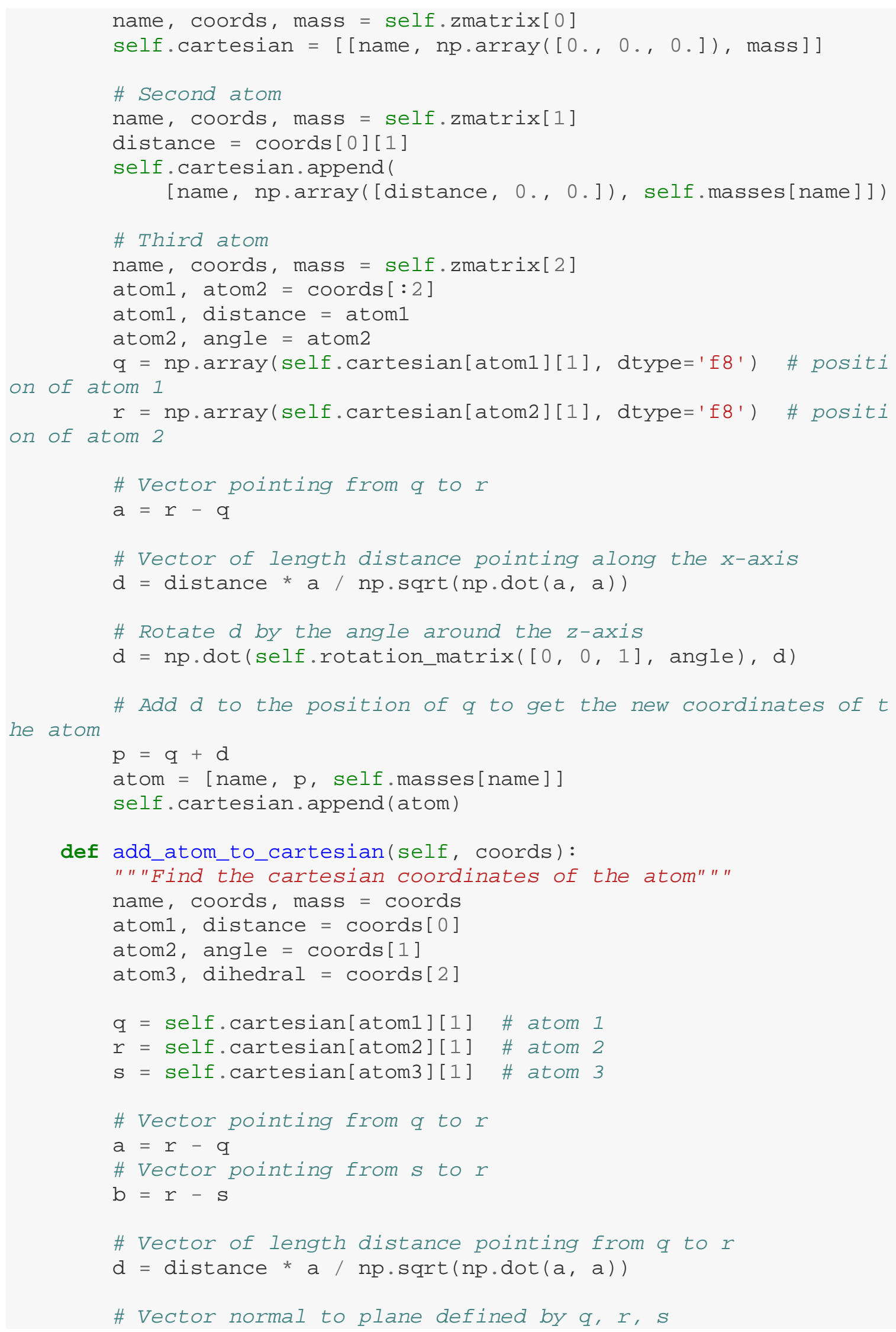


normal $=n p \cdot \operatorname{cross}(a, b)$

\# Rotate $d$ by the angle around the normal to the plane defin ed by $q, r, s$

$\mathrm{d}=\mathrm{np} \cdot \operatorname{dot}(\operatorname{self} \cdot \operatorname{rotation}$ matrix(normal, angle $), \mathrm{d})$

\# Rotate $d$ around a by the dihedral

$d=n p \cdot \operatorname{dot}($ self.rotation_matrix(a, dihedral), d)

he atom

\# Add $d$ to the position of $q$ to get the new coordinates of $t$

$p=q+d$

atom $=[$ name, $p$, mass $]$

self. cartesian . append (atom)

def zmatrix_to_cartesian(self):

"I" "

Convert the zmartix to cartesian coordinates

"I" "

\# Deal with first three line separately

self.add_first_three_to_cartesian( )

for atom in self.zmatrix[3:]:

self.add_atom_to_cartesian(atom)

\#self.remove_dummy_atoms()

self.center_cartesian( )

return self.cartesian

def add_first_three_to_zmatrix(self):

"" "The first three atoms need to be treated differently"""

\# First atom

self.zmatrix $=[]$

name, position, mass = self. $\operatorname{cartesian}[\odot]$

self.zmatrix.append([name, [[], [], []], mass])

\# Second atom

if len(self.cartesian) > 1:

name, position, mass = self.cartesian[1]

atom1 = self. $\operatorname{cartesian[0]}$

pos1 = atom1 $[1]$

$q=$ pos1 - position

distance $=n p \cdot \operatorname{sqrt}(n p \cdot \operatorname{dot}(q, q))$

] ) self.zmatrix.append([name, $[[\Theta$, distance $],[],[]]$, mass

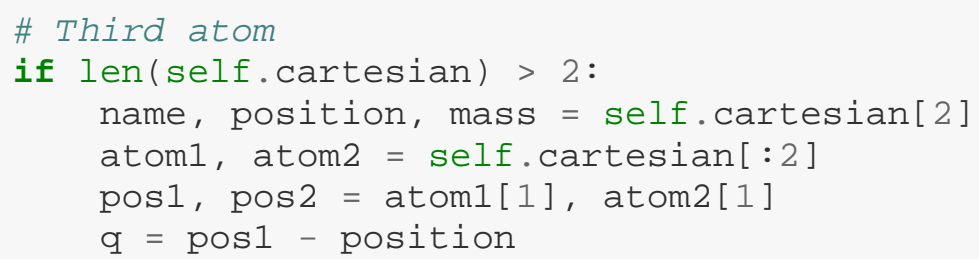


mass ])

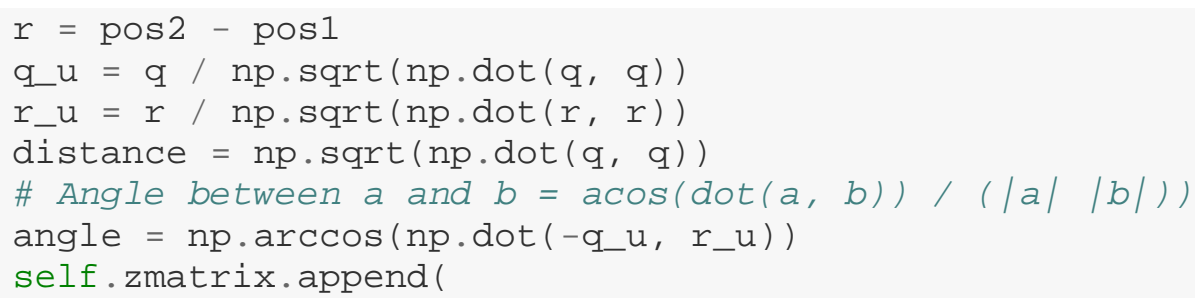


def remove_dummy_atoms(self):

"" Delete any dummy atoms that may have been placed in the $c$ alculated cartesian coordinates"" "

new_cartesian $=[]$

for atom, xyz, mass in self.cartesian:

if not atom $==$ ' $X$ ':

new_cartesian.append((atom, xyz, mass))

self. cartesian = new_cartesian

def center_cartesian(self):

"" "Find the center of mass and move it to the origin"""

self.total_mass $=0.0$

center_of_mass $=\mathrm{np} . \operatorname{array}([\odot . \odot, \odot . \odot, \odot . \odot])$

for atom, $x y z$, mass in self.cartesian:

self.total_mass $+=$ mass

center_of_mass $+=x y z$ * mass

center_of_mass = center_of_mass / self.total_mass

\# Translate each atom by the center of mass

for atom, xyz, mass in self.cartesian:

$x y z-=$ center_of_mass

def cartesian_radians_to_degrees(self):

for atom in self.cartesian:

atom[1][1][1] = np.degrees(atom[1][1][1])

atom[1][2][1] = np.degrees $(\operatorname{atom}[1][2][1])$

def output_cartesian(self, output_file='cartesian.dat'):

"""Output the cartesian coordinates of the file"""

with open(output_file, ' $w+'$ ) as $f$ :

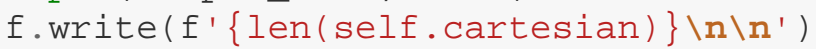

f.write(self.str_cartesian( ))

def str_cartesian(self):

"" "Print the cartesian coordinates"""

out $=1$ '

for atom, (x, y, z), masses in self.cartesian:

out $+=f^{\prime}\{$ atom: $<2 s\} \quad\{x:>15.10 f\} \quad\{y:>15.10 f\} \quad\{z:>15.10 f\} \backslash$

$n^{\prime}$

return out

def output_zmatrix(self, output_file='zmatrix.dat'):

"""Output the zmatrix to the file"""

with open(output_file, ' $w+'$ ') as $f$ :

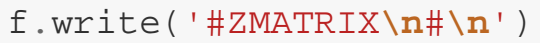

f.write(self.str_zmatrix())

def str_zmatrix(self):

"" "Print the zmatrix"""

out $=f^{\prime}\{$ self.zmatrix $[0][0]\} \backslash n^{\prime}$

for atom, position, mass in self.zmatrix[1:]:

out $+=f^{\prime}\{$ atom: $<2 s\}$ ' 


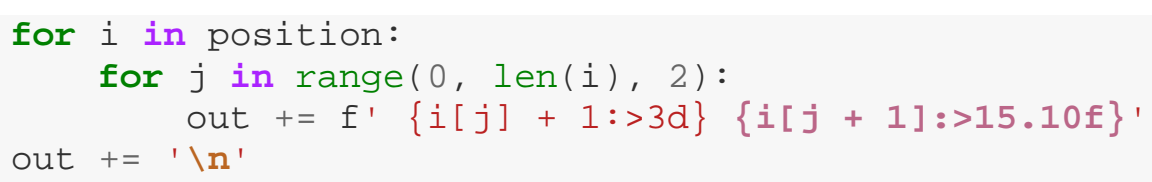

def run_zmatrix(self, input_file='zmatrix.dat', output_file='car tesian.dat' '):

"" "Read in the zmatrix, converts it to cartesian, and output $s$ it to a file""l" self.read_zmatrix(input_file) self.zmatrix_to_cartesian() self.output_cartesian(output_file)

def run_cartesian(self, input_file='cartesian.dat', output_file= 'zmatrix.dat'):

"""Read in the cartesian coordinates, convert to cartesian, and output the file""'"

self.read_cartesian(input_file)

self.cartesian_to_zmatrix()

self.output_zmatrix(output_file)

$z=$ Converter ()

z.read_zmatrix(input_file="Ca-methoxide_SA_ST0_1.gjf")

z.zmatrix_to_cartesian( )

\#z.remove_dummy_atoms()

z.output_cartesian() 


\section{CURRICULUM VITAE}

Anam Chandra Paul

2206 James Guthrie Ct,

Apt \#4 | Louisville, KY, 40217

502-851-7894

acpaul04@louisville.edu/anamchandrapaul@gmail.com

\section{EDUCATION}

Ph.D. in Physical Chemistry, Fall-2019, Supervisor - Dr. Jinjun Liu

University of Louisville

Dissertation Title: "Laser spectroscopic investigations of jet-cooled metals containing diatomic and polyatomic free radicals.”

MSc in Physical Chemistry, Fall-2016, Supervisor - Dr. Jinjun Liu

University of Louisville

Cumulative G.P.A. - 3.88

Thesis: "Laser spectroscopic investigations of jet-cooled calcium alkoxides free radicals.”

BSc in Chemistry, June - 2013

University of Dhaka

Cumulative G.P.A. - 3.35

Project Supervisor - Dr. Mohammed Shoeb 
Undergrad Project: "Studies of polycyclic aromatic hydrocarbons (PAHs) in lake water samples in Dhaka city area."

\section{Laboratory Skills}

- Extensive experience in tuning and operating various laser systems including pulsed and continuous mode.

- Vast knowledge of different types of spectroscopic equipment and component including, monochromators, wavemeters, filters, photodiodes, photomultiplier tubes, lenses, and mirrors.

- Trained in various spectroscopic experiments including laser ablation/molecular jet, laser photolysis, pulsed electric discharge, laser-induced fluorescence (LIF)/ dispersed fluorescence (DF), cavity ring-down (CRD).

- Broad understanding of high vacuum system, delay generators, high voltage power supplies, waveform generators, and lock-in-amplifiers.

\section{Experience}

\section{Research Assistant}

Department of Chemistry, University of Louisville, Louisville, Kentucky

- Repaired, tuned and operated various laser systems including, Spectra physics Nd: YAG laser, GCR Nd: YAG, Continuum (precision II) YAG laser, Precision scan Sirah dye laser, Different frequency mixer (DFM) Sirah to generate 1.4 to $3.5 \mu \mathrm{m}, \mathrm{H}_{2}$ Raman shifter.

- Conducted laser excitation studies in the visible regions using a laser ablation molecular jet source.

- Designed motorized metal sample holders for laser ablation in the pulsed nozzle for metal-containing radicals generations in the jet.

- Obtained, processed and analyzed moderate resolution and high-resolution spectra of various molecules including nonlinear polyatomic, symmetric tops. 
- Tuned and operated various laser systems including Titanium sapphire( M-squared, Coherent 899-29), ring dye( Coherent 699-29), Coherent Verdi(V10), CW OPO(Toptica), Excimer (Lamda Physik, LPX-120).

\section{Visiting Scholar}

Non-equilibrium thermodynamics laboratory, The Ohio State University, Columbus, Ohio

- Repaired, tuned and operated dye laser system for cavity ring-down spectroscopy

- Worked in the CRDS system using flow cell in room temperature.

\section{Teaching Assistant}

- Department of Chemistry, University of Louisville, Louisville, Kentucky

- Coordinated tutorial sessions for general chemistry courses.

- Lectured to several recitations (Chem 101,201, 202) classes of approximately 30 students in each section.

- Helped course instructor in setting up labs intro chemistry laboratory (Chem 103)

\section{Research Assistant}

Department of Chemistry, University of Dhaka, Dhaka, Bangladesh

- Processed lake water samples for gas chromatography

- Experienced in various analytical apparatus including GC, GC-MS, UV-VIS etc.

\section{Technical Skills}

- Quantum Chemistry Codes: Gaussian and Cfour

- Visualization software: Gaussview, chemcraft and chemdraw

- Plotting software and data analysis: Origin and Matlab

- Programming languages: Basic knowledge of Python and LabVIEW(beginner level)

- CAD software: SolidWorks, Auto-CAD, On shape(online software) 


\section{PUBLICATIONS (PEER-REVIEWED JOURNALS)}

1.) "Dispersed-fluorescence spectroscopy of jet-cooled calcium ethoxide radical ( $\left.\mathrm{CaOC}_{2} \mathrm{H}_{5}\right)$ ", A. C. Paul, Md. A. Reza, and J. Liu,* J. Mol. Spectrosc.330, 142-146 (2016). DOI: https://doi.org/10.1016/j.jms.2016.09.001

2.) "Dispersed fluorescence spectroscopy of jet-cooled iso-butoxy and 2-methyl-1-butoxy radicals”, Md. A. Reza, A. C. Paul J. Alam, N. J. Reilly, and J. Liu,* J. Phys. Chem. A 120, 6761-6767 (2016). DOI: https://doi.org/10.1021/acs.jpca.6b06445

3.) "Direct observation of tetrahydrofuranyl and tetrahydropyranyl peroxy radicals via cavity ring-down spectroscopy”, H. Telfah, Md. A. Reza, J. Alam, A. C. Paul and J. Liu,* J. Phys. Chem. Lett. 9, 4475-4480 (2018).

DOI: https://doi.org/10.1021/acs.jpclett.8b01721

4.) "Laser-induced fluorescence and dispersed-fluorescence spectroscopy of the ${ }^{\sim} A^{2} E$ $\sim X^{2} \mathrm{~A}_{1}$ transition of jet-cooled calcium methoxide $\left(\mathrm{CaOCH}_{3}\right)$ Radicals" A. C. Paul, K. Sharma, Md. A. Reza, H. Telfah, T. A. Miller,* and J. Liu,* J. Chem. Phys. 151, 134303 (15 pages) (2019). DOI: https://doi.org/10.1063/1.5104278

5.) "Laser-induced fluorescence and dispersed fluorescence spectroscopy of jet-cooled isopentoxy radicals" Md. A. Reza, A. C. Paul, N. Reilly, and J. Liu,* J. Phys. Chem. A 123, 8441-8447 (2019). DOI: https://doi.org/10.1021/acs.jpca.9b07307

\section{Manuscript in preparation}

1.) "Laser-induced fluorescence and dispersed-fluorescence spectroscopy of the ${ }^{\sim} A^{2} E$ ${ }^{\sim} \mathrm{X}^{2} \mathrm{~A}_{1}$ transition of jet-cooled calcium ethoxide and -isopropoxide $\left(\mathrm{CaOC}_{2} \mathrm{H}_{5}\right.$ and CaOCH$\left.\left(\mathrm{CH}_{3}\right)_{2}\right)$ Radicals" A. C. Paul, K. Sharma, T. A. Miller,* and J. Liu,* Manuscript in preparation. 
2.) “Cavity ring-down spectroscopy of jet-cooled YO radicals” A. C. Paul, H. Telfah, J. Liu, Manuscript in preparation.

\section{ORAL PRESENTATIONS (SPEAKER UNDERLINED)}

June 2019

June 2019

June 2018
ANAM C PAUL, HAMZEH TELFAH, JINJUN LIU, CAVITY RING-DOWN SPECTROSCOPY OF JET-COOLED YTTRIUM OXIDE $74^{\text {th }}$ International Symposium on Molecular Spectroscopy, Champaign-Urbana, Illinois, USA

KETAN SHARMA, ANAMA C. PAUL, JINJUN LIU, TERRY A MILLER ANALYSIS OF PSEUDO-JAHN-TELLER EFFECT IN METAL CONTAINING ALKOXIDES

$74^{\text {th }}$ International Symposium on Molecular Spectroscopy, Champaign-Urbana, Illinois, USA

ANAM C PAUL, MD ASMAUL REZA, PRANOY DEB SHUVRA, JINJUN LIU

LASER-INDUCED FLUORESCENCE AND DISPERSEDFLUORESCENCE SPECTROSCOPY OF JET-COOLED

\section{CALCIUM MONOALKOXIDE RADICALS}

$73^{\text {rd }}$ International Symposium on Molecular Spectroscopy, Champaign-Urbana, Illinois, USA

ANAM C PAUL, MD ASMAUL REZA, KETAN SHARMA, TERRY A. MILLER, JINJUN LIU,

ALKALINE EARTH MONOALKOXIDE FREE RADICALS AS CANDIDATES FOR LASER COOLING OF POLYATOMIC MOLECULES 
$73^{\text {rd }}$ International Symposium on Molecular Spectroscopy, Champaign-Urbana, Illinois, USA

HAMZEH TELFAH, MD ASMAUL REZA, ANAM C PAUL, JINJUN LIU

OBSERVATION OF THE $\tilde{A} \leftarrow \tilde{X^{2}}$ ELECTRONIC

TRANSITIONS OF TETRAHYDROPYRANYL AND

TETRAHYDROFURANYL PEROXY RADICALS BY ROOM-

TEMPERATURE CAVITY RING-DOWN SPECTROSCOPY

$73^{\text {rd }}$ International Symposium on Molecular Spectroscopy

Champaign-Urbana, Illinois, USA

MD ASMAUL REZA, HAMZEH TELFAH, ANAM C PAUL, JAHANGIR ALAM, JINJUN LIU.

CAVITY RING-DOWN SPECTROSCOPY OF 1-, 2- AND 3-

METHYL ALLYL PEROXY RADICALS

$73^{\text {rd }}$ International Symposium on Molecular Spectroscopy, Champaign-Urbana, Illinois, USA

June 2017

ABDELQADER JAMHAWI, ANAM C PAUL, JUSTIN D.

SMITTH, SACHIN HANDA, JINJUN LIU,

ULTRAFAST TRANSIENT ABSORPTION SPECTROSCOPY

OF POLYMER-BASED ORGANOPHOTOREDOX

CATALYSIS MIMIKING TRANSITION-METAL

COMPLEXES

$72^{\text {th }}$ International Symposium on Molecular Spectroscopy,

Champaign-Urbana, Illinois, USA

June 2016

ANAM C. PAUL, MD ASMAUL REZA, JINJUN LIU, DISPERSED-FLUORESCENCE SPECTROSCOPY OF JET-

COOLED CALCIUM ETHOXIDE RADICAL (CaOC2H5) 
$71^{\text {th }}$ International Symposium on Molecular Spectroscopy, Champaign-Urbana, Illinois, USA

\section{AWARDS}

- International Student Tuition Support Award, University of Louisville (\$200) 2018

- The Graduate Network in Arts and Science (GNAS) Travel Award, (\$250) 2018

- Department of Chemistry Travel Award, (\$200) 2017

- Banghabondhu Fellowship, Bangladesh (60000 Tk) 2014 